\title{
Research at LAMPF
}

February, $1992 \quad \begin{aligned} & \angle A-4 R-92-926 \\ & \text { C.I }\end{aligned}$

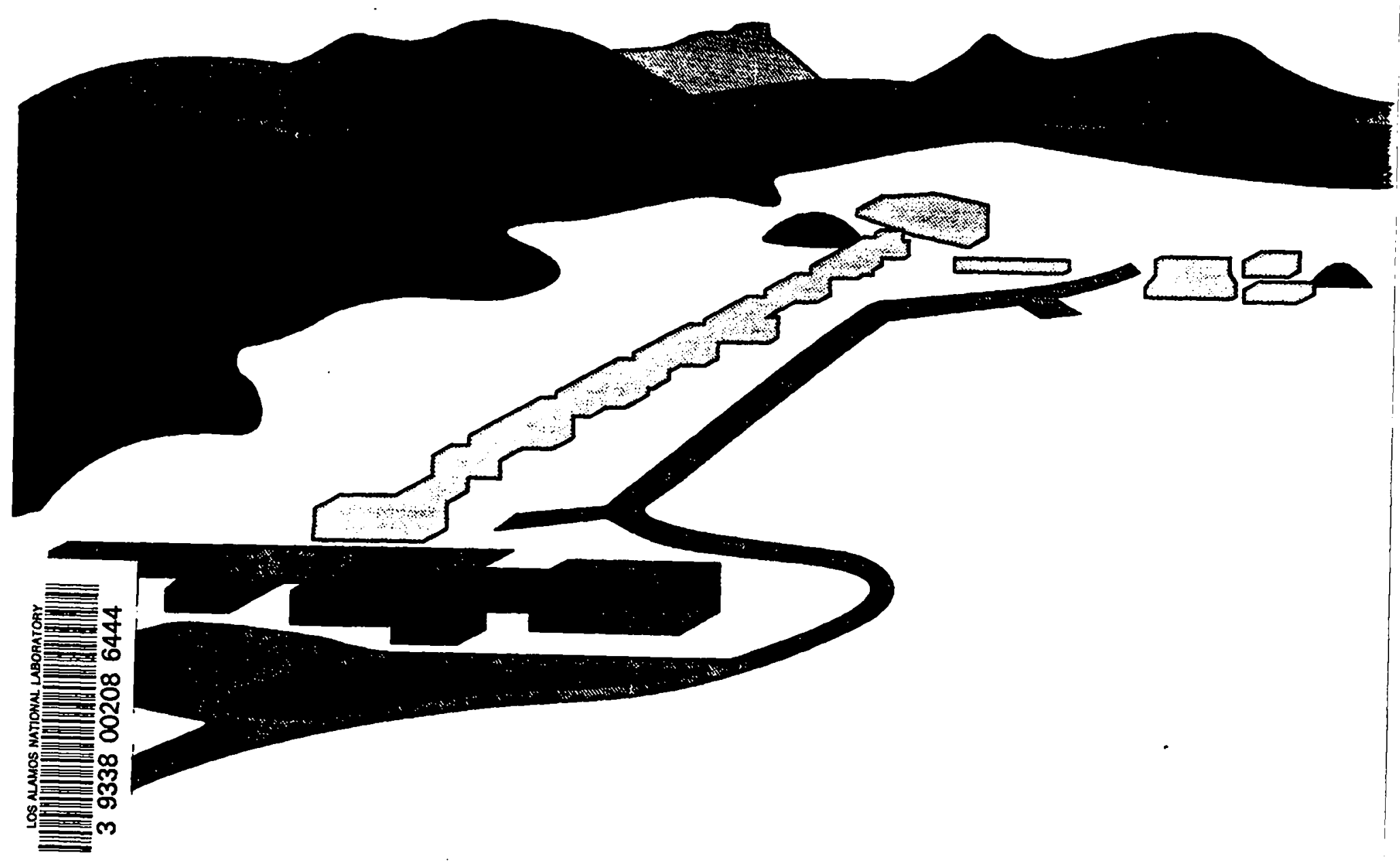


Los Alamos WATIONAL LABO OAATORY

\section{memorandum}

Medium Energy Physics Division MP-4, Nuclear and Particle Physics
ToMS: Jack Carter, IS-4, P364

Fromms: Karen Poelakker Karen Palabker Phone/FAX: 7-6941/FAX 5-1712

Symbol: MP-4-92-030

Date: April 15, 1992

Copy of Research at LAMPF for Report Library

Jan Gallegos suggested that I send you a copy of Research at LAMPF, LA-UR-92-926, for your

Report Library. If you receive requests for this document, please direct the requests to me at MP-4.

Cy: Jan Gallegos, OS-6, F674

MP-4 File 


\section{Foreword}

During the past decade, both the science program and the experimental facilities at LAMPF have undergone some significant changes. This report is a concise description of the current research initiatives and of new directions being proposed for the future research program at LAMPF.

Traditionally the LAMPF physics program has explored nuclear systems with hadronic probes: pions, protons, and neutrons. In recent years the exploitation of the intense lepton beams - neutrinos and muons - at LAMPF has received increasing emphasis. Explorations of issues in electroweak physics and precision tests of quantum electrodynamics have been significant areas of experimentation. A new component of the program is an increased emphasis on the role of weak interactions in hadronic systems. Here the electroweak force is treated as a well-understood interaction, which can be used to reveal the wave functions and properties of hadronic systems. The manifestations of the electroweak force may be discussed either in the language of quarks interacting through the exchange of $\mathrm{W}$ and $\mathrm{Z}$ bosons or in terms of nucleons interacting through the exchange of mesons described by both strong and weak coupling constants.

Taken together, these topics provide a rich and vital program of fundamental interest in nuclear physics. Atomic physics, astrophysics, materials research, among others, are additional components of the LAMPF-LANSCE experimental activities. Research highlights of this broad program and the research tools available at LAMPF are described briefly in the following pages.

Research at LAMPF has been developed largely by a team of physicists at Los Alamos National Laboratory but is based on the ideas, plans, and accomplishments of a large group of users, both inside and outside the laboratory, who have committed years of effort toward developing an effective and productive program in nuclear physics at LAMPF. Specific acknowledgement should be made of the special contribution of R. Bolton, J. Bowles, B. Keffeler, K. Poelakker, and C. Weaver. I thank them all and especially C. Hoffman, who coordinated this activity, gave it a clear focus, and generated this final report.

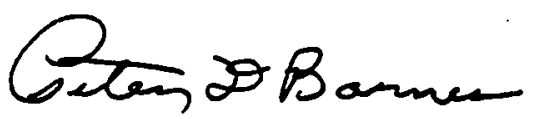

Peter D. Barnes

Director of LAMPF

February 20, 1992 


\section{Table of Contents}

I. Introduction

II. Strong Interaction Physics ......................................................................................I-1

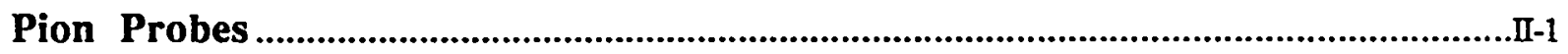

Introduction ........................................................................................................................I-1

Recent Accomplishments and the Ongoing Program .................................................I-2

Chiral Symmetry Breaking.............................................................................I-2

Chiral Symmetry Breaking, $\pi N$ Scattering....................................................II-2

Chiral Symmetry Breaking, $\pi \pi$ Scattering ...................................................II-2

Nuclear Structure...............................................................................................I-2

Determination of the Neutron Distributions in Nuclei ..........................................I-3

Exploration of Isospin Mixing in Nuclear States.................................................-3

Measurement of Nuclear Transition Effective Charges ........................................I-3

Characterization of Stretched States..................................................................II-3

Discovery of the Giant Isovector Monopole Resonance........................................I-3

Discovery of Double Resonances in Nuclei .......................................................I-4

Creation of Exotic Nuclei ................................................................................

Investigation of NN Correlations in Nuclei .....................................................I-4

Hadrons in the Nuclear Medium.........................................................................I-5

Pion Absorption ..............................................................................................

Pion Propagation .........................................................................................

$\Delta$ Propagation and Interactions..................................................................I-

Pion-Nucleus Transparency .......................................................................I-8

Inclusive DCX...................................................................................

Quasi-Free Knockout Reactions .................................................................I-8

$\eta$ Production ........................................................................................

The Near-Term Program at LAMPF ..........................................................................I-9

The Pion-Nucleon Program ...............................................................II-9

Pion Single Charge Exchange....................................................................

Pion-Nucleus Double Charge Exchange .................................................................9

Quasi-Free Reactions............................................................................................

Pion Scattering from Polarized Nuclear Targets...........................................I-10

Absorption of Pions in Nuclei .....................................................................1-10

Exotic Nuclei.................................................................................................1I1

Summary ............................................................................................................11 


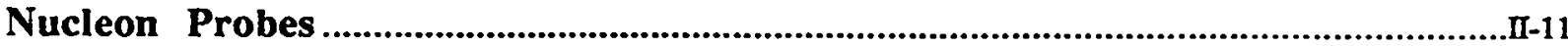

Accomplishments and Opportunities.........................................................................11

The Spin-Longitudinal and Spin-Transverse Response of the Nucleus..........................I-12

Pionic Correlations and the Delta Resonance ...............................................................I-13

Medium Modifications of Hadrons in the Nucleus ......................................................I-14

Elastic Scattering and Scattering Theory ....................................................................1-15

The Nucleon-Nucleon Program at LAMPF ..................................................................I-16

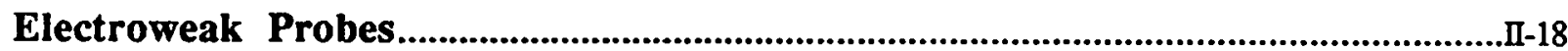

Introduction .................................................................................................................18

Measuring the Quark Contribution to the Proton Spin through $v p \rightarrow v p . . . . . . . . . . . . . . . . . . . . . I-18$

vp $\rightarrow$ vp Formalism.......................................................................................18

The LSND Experiment ..................................................................................I-19

Study of the Induced Pseudoscalar Form Factor using Polarized ${ }^{3}$ He..............................I-20

Neutrino Carbon Neutral Current Interactions..............................................................I-21

Experimental Study of Neutrino Absorption on Carbon ................................................I-21

Parity and Time-Reversal Violation in the Compound Nucleus......................................I-23

Parity Nonconservation in Proton Scattering ...............................................................I-24

Study of the Weak Radiative Decay $\pi \rightarrow e v \gamma$...............................................................26

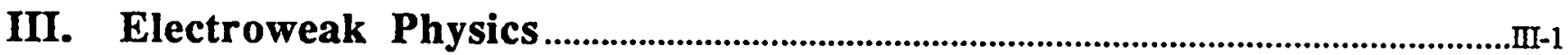

Precision Tests of the Standard Model...........................................................................1

Introduction ..........................................................................................................................

Measurement of the Michel Parameters with the MEGA Positron Spectrometer..............II-1

Pion Beta Decay........................................................................................................II-2

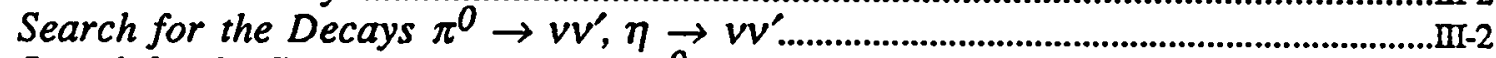

Search for the C-Noninvariant Decay $\pi^{D} \rightarrow$ MY.......................................................II-3

Muonium and Muonic Atoms ........................................................................................m-3

E225: Neutrino Electron Scattering and Neutrino Properties..........................................II-3

The Family Number Problem..........................................................................................m-5

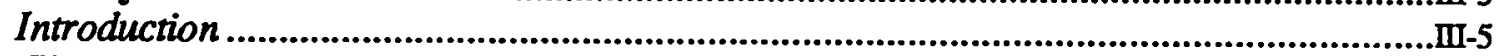

Character of the Conservation Law............................................................................II-5

Mixing in the Lepton Sector........................................................................................II-6

Family Transitions ..................................................................................................II-8

New Particles..............................................................................................................10

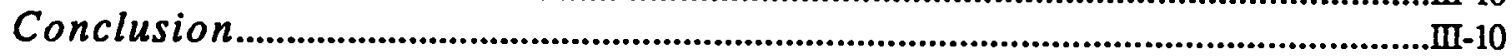

IV. Additional Basic and Applied Research.........................................................1

Introduction .................................................................................................................

Mass Measurements and Exotic Decay Studies with TOFI ......................................

Introduction ............................................................................................................

TOFI Mass Measurement Program ................................................................................

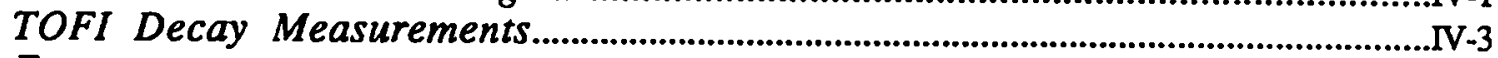

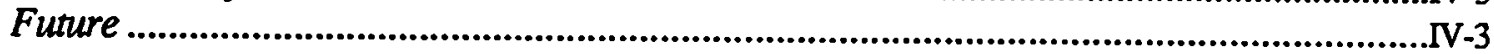




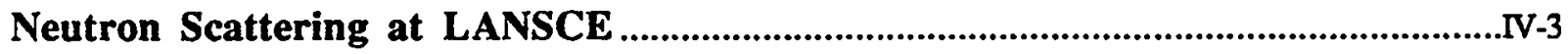

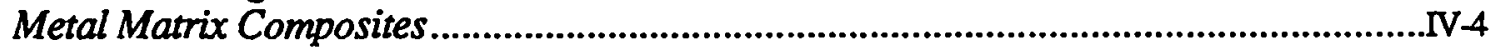

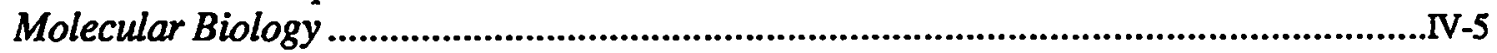

Polymers....................................................................................................................

Structure of Rare-Earth Oxides ................................................................................

Nuclear Physics Studies with Neutrons at LANSE and WNR ...........................V-6

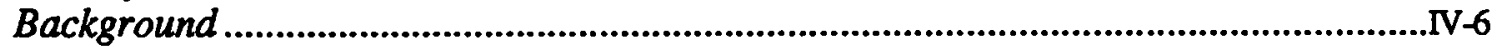

Ongoing Research...................................................................................................6

Parity and Time-Reversal Tests in Compound Nuclei..................................IV-6

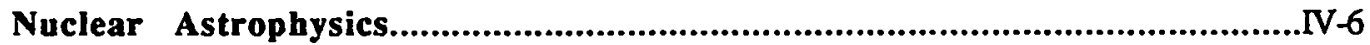

Neutron-Proton Bremsstrahlung.................................................................

Gamow-Teller and Giant Resonance Excitations in (n,p) Reactions.............IV-7

Proposed Future Research .....................................................................................

Isotropic Polarized Thermal Neutron Source ...............................................IV-7

Dibaryon Resonance ...............................................................................

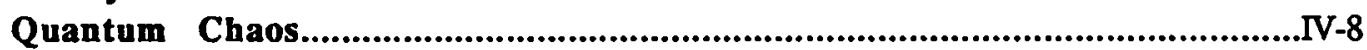

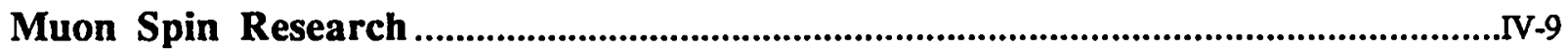

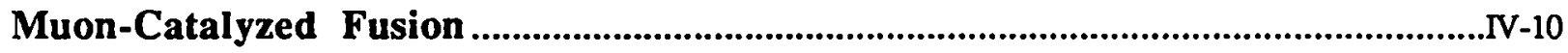

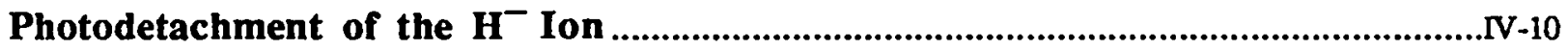

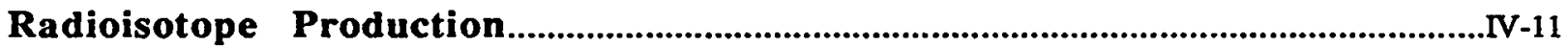

Commercial LAMPF-Produced Radioisotopes ...............................................................

Research Uses of LAMPF-Produced Radioisotopes ................................................12

Studies of the Effects of Radiation on Materials...................................................

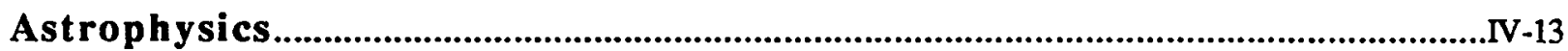

Ultra-High-Energy Gamma-Ray Astronomy ...........................................................V-13

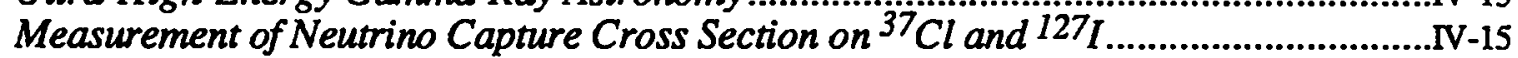

Detection of Neutrinos from Supernovae........................................................................

Measurement of Cross Sections of Astrophysical Interest ........................................IV-15

Thin Target He-Jet System.....................................................................................IV-15

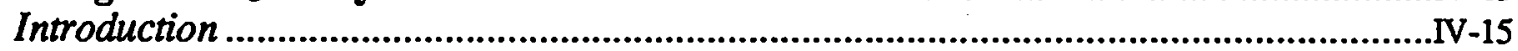

A Thin Target He-Jet System at the LAMPF Beam Stop ..............................................

Research Program........................................................................................................

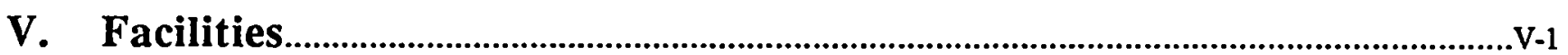

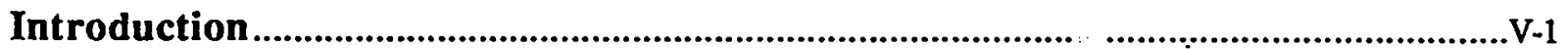

Energetic Pion Channel and Spectrometer (EPICS) ..................................................

Large Acceptance Spectrometer (LAS) ..............................................................

Scruncher/Clamshell .........................................................................................V-2 


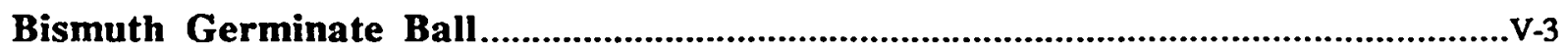

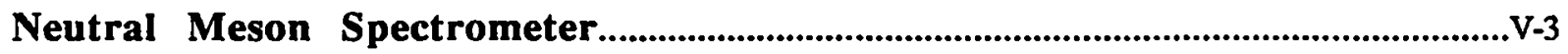

The Optically Pumped Polarized Ion Source (OPPIS) .......................................

Neutron Time-of-Flight Facility (NTOF) ................................................................

High Resolution Spectrometer (HRS) .................................................................. V-4

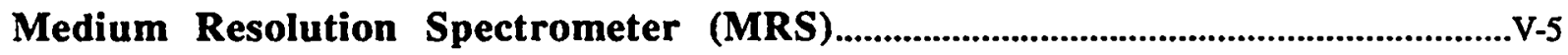

Polarized Neutron Area .........................................................................................

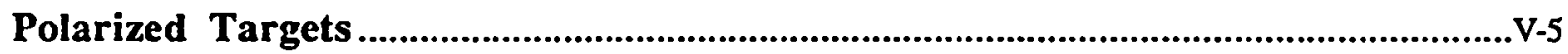

High Resolution Atomic Beam Facility ..................................................................

Time-of-Flight Isochronous Spectometer (TOFI)..................................................

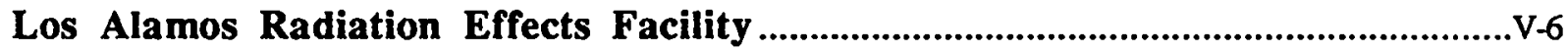

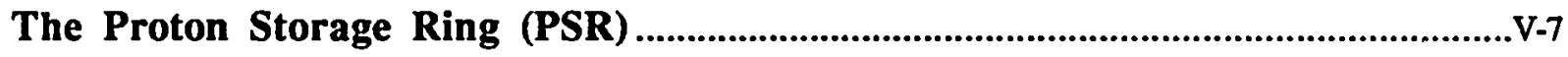

The Manual Lujan Jr. Neutron Scattering Center (LANSCE) .................................V-7

Volume Production Multicusp $\mathrm{H}^{-}$Ion Source .........................................................

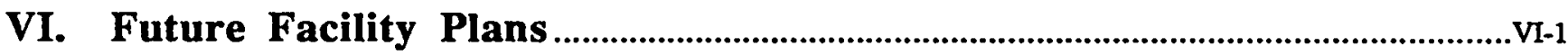

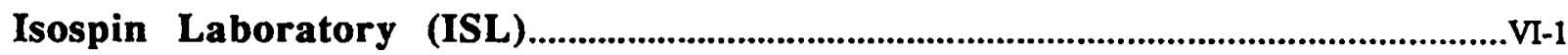

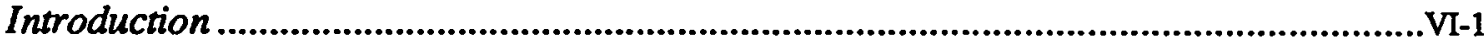

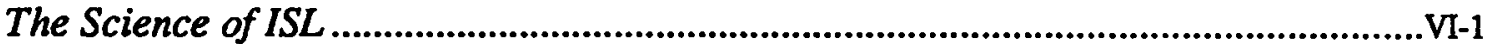

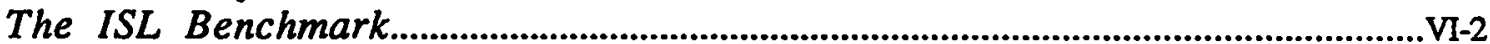

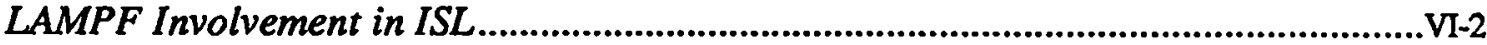

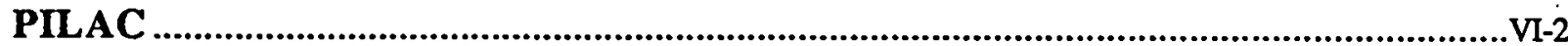

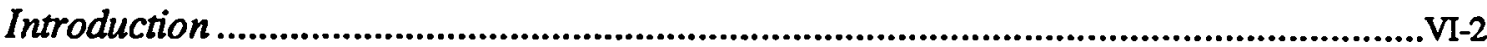

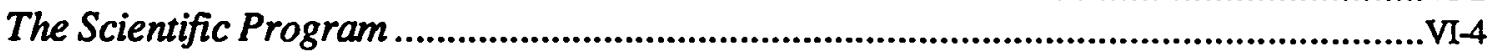

Lambda Hypernuclei ............................................................................

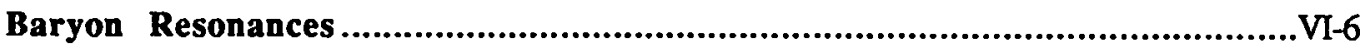

High-Energy Pion-Nucleus Reactions .................................................VI-6

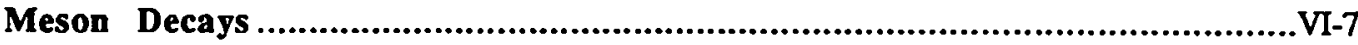

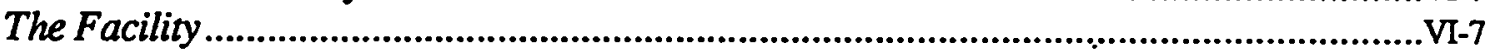

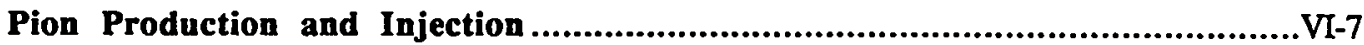

The Superconducting Pion Linac...................................................................

Beam Lines and Experimental Facilities.......................................................VI-9

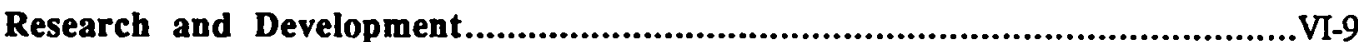

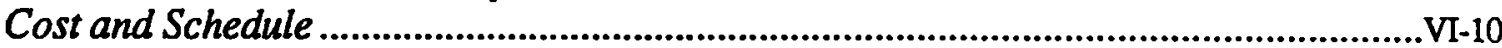

Summary ......................................................................................................... 
Pulsed Lepton Source (PLS) VI-11

Fundamental Muon Physics

Neutrino Physics

Materials Science with Pulsed Muons.

Ultracold Neutrons...............................................................................................................14

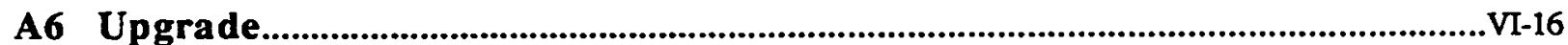

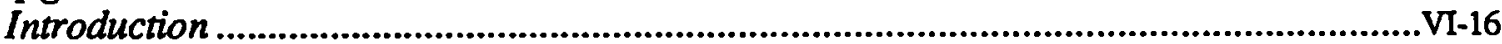

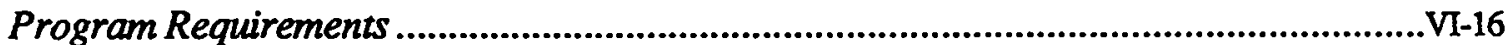

Reference Design....................................................................................................VI-17

Appendix A. Research Highlights of Last Five Years

Appendix B. LAMPF Facility Milestones 


\section{Introduction}

Research at the Los Alamos Meson Physics Facility (LAMPF) is a multidisciplinary activity encompassing nuclear physics, elementary particle physics, atomic and molecular physics, astrophysics, condensed matter physics, and nuclear chemistry. In addition, studies of radiation damage to materials and to electronics components are carried out, and a number of important radioisotopes for commercial and research use are uniquely produced at LAMPF. LAMPF research has resulted in about 1200 papers in refereed journals, based upon the completion of about 700 experiments. Over $200 \mathrm{Ph} . \mathrm{D}$. theses have come from research at LAMPF. LAMPF is a national user facility with an 800 -member users group representing over 100 U.S. and foreign universities. Each year, about 30 experiments receive beam and approximately 400 scientists visit the facility to plan, carry out, and analyze these experiments. At present, there are 92 approved experiments awaiting beam time resulting in long queues in the beam channels.

The heart of the facility is an $800-\mathrm{MeV}$ proton linac that accelerates $\mathrm{H}^{+}, \mathrm{H}^{-}$, and polarized $\mathrm{H}^{-}$beams. The figure at the end of this introduction gives a schematic view of the LAMPF complex. The 1-mA $\mathrm{H}^{+}$beam current makes LAMPF the highest power proton accelerator in the world. The primary proton beam produces intense, high-quality secondary beams of pions, muons, and neutrinos. At the same time the $\mathrm{H}^{+}$is accelerated, LAMPF can produce variable-energy polarized proton beams for studies of spin-dependent nucleon scattering, and intense bunches of $\mathrm{H}^{-}$ions for injection into the high-current 800-MeV proton storage ring (PSR) and to a high-intensity white neutron source (WNR). PSR generates pulsed beams of neutrons at the Los Alamos Neutron Scattering Center (LANSCE). Typically 7 or 8 nuclear physics experiments are simultaneously collecting data during LAMPF operations, including 1 or 2 at LANSCE and WNR. At the same time, another 10 experiments in other disciplines are also receiving beam at the latter two facilities.

The 1989 NSAC Long Range Plan lays out the central goals of nuclear physics for the decade of the ' $90 \mathrm{~s}$ and highlights a number of examples of how these goals can be met. Many elements of the present and future research program at LAMPF directly address these goals, including:

- Studies of pion absorption in nuclei.

- Separation of the spin-longitudinal and spintransverse response of the nucleus with $(p, n)$ polarization studies.

- A study of v-p elastic scattering to measure the strange quark contribution to the proton spin.

- The investigation of parity- and time-reversalinvariance in neutron scattering from heavy nuclei.

- Investigations of chiral symmetry breaking in pion scattering.

- Sensitive searches for rare decays and neutrino oscillations that would indicate mixing between lepton families.

- Study of the structure of hypernuclei with high resolution.

- Studies of nuclear transparency in single charge exchange.

- Measurements of masses and decays of nuclei far from the limits of stability.

- Precision tests of the Minimal Standard Model.

- Studies of deltas in the nuclear medium.

- The use of radioactive esams to measure cross sections needed for an understanding of nucleosynthesis.

- The use of neutrino/muon detectors in the study of ultra-high-energy gamma-ray emission from astronomical objects such as Cygnus X-3 and Hercules X-1. 
- Development of an intense source of cold and ultracold neutrons for fundamental measurements.

- A precise measurement of the weak mixing angle, $\theta \mathrm{W}$, and of electroweak radiative corrections in atomic parity measurements utilizing a helium-jet atomic beam facility.

- Measurement of the hyperon-nucleon weak force.

- Study of NN correlations in nuclei with double charge exchange.

- Measurement of hadronic form factors with electroweak probes.

- Study of giant resonances and double giant resonances in pion and nucleon scattering.

- The detection of neutrinos from supemovae.

- Determination of nucleon-nucleon scattering amplitudes.
- The measurement of the neutrino capture cross section on chlorine to calibrate the Homestake solar neutrino experiment.

- Study of hypernuclear states with large polarizations using the $\left(\pi^{+}, \mathrm{K}^{+}\right)$reaction.

- Studies of the chaotic nature of levels in compound nuclei with epithermal neutrons.

- Detailed study of spin-dependent and spinindependent excitation modes in nuclei.

What follows is a concise discussion of research at LAMPF including highlights of past research, an examination of the present research program, and a look at the directions the program will take in the near future. Also included are brief descriptions of the major experimental tools used to carry out this research, and of upgrades that are planned for the near future. 


\section{LAMPF \\ Experimental Areas}
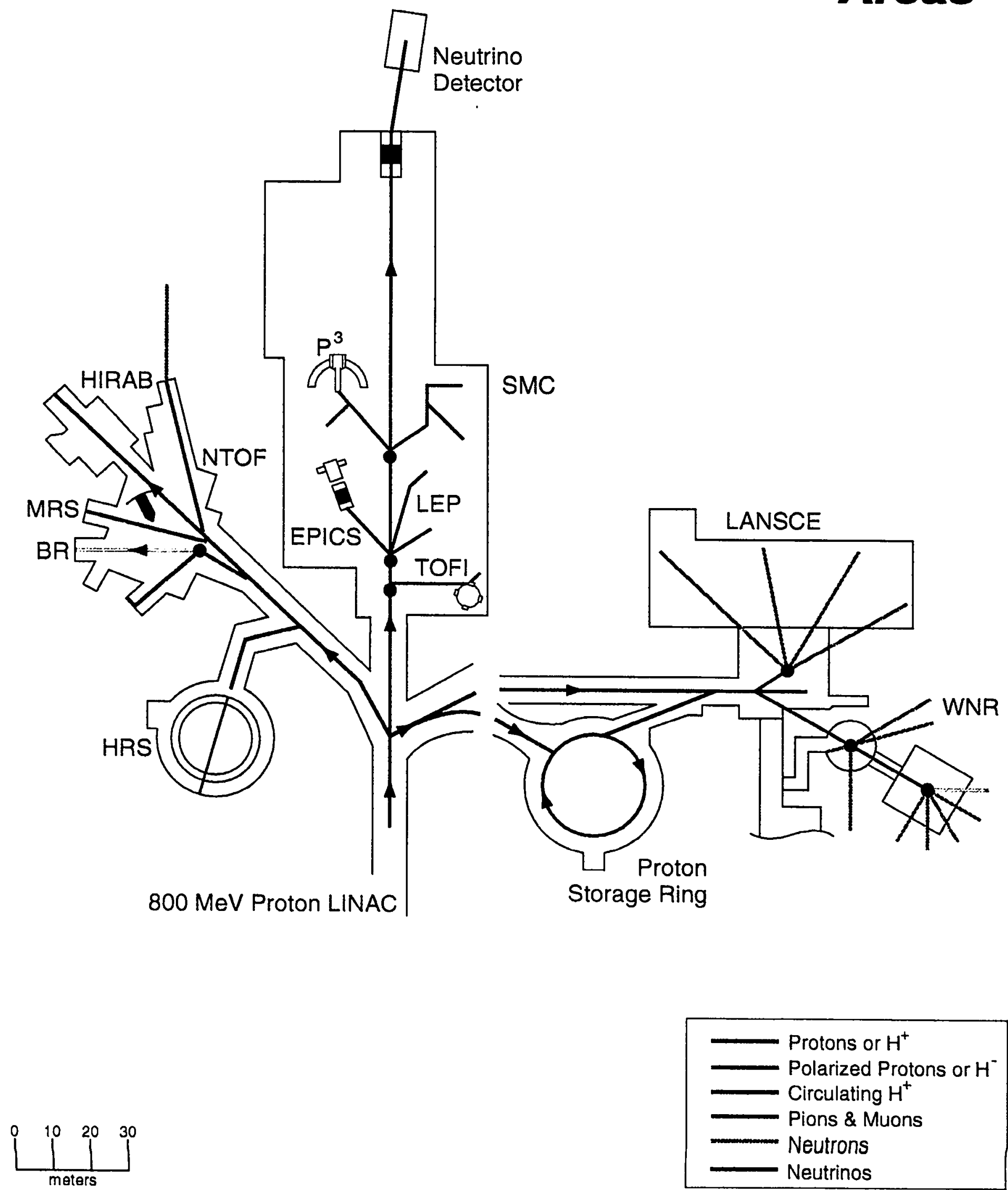

\begin{tabular}{|l}
- Protons or $\mathrm{H}^{+}$ \\
Polarized Protons or $\mathrm{H}^{-}$ \\
Circulating $\mathrm{H}^{+}$ \\
Pions \& Muons \\
Neutrons \\
Neutrinos
\end{tabular}




\section{Strong Interaction Physics}

\section{Pion Probes}

\section{Introduction}

The Los Alamos Meson Physics Facility plays a central role in strong interaction physics. This is not surprising, owing to its array of high-intensity beams, modern detector systems, and the skill, vitality, and enthusiasm of those who use them. The pion plays an important role in strong interaction physics, entering almost every aspect of medium-energy nuclear physics. Its interaction with the nucleon also provides the basic data for characterization of the baryon mass spectrum and for understanding how chiral symmetry is broken in the QCD Lagrangian.

As a boson, the pion can be absorbed and created in nuclei. It can produce other mesons and excite baryon resonances in the nucleus. Pion absorption in nuclei is emphasized in the current NSAC Long Range Plan as one of the "most intriguing problems." Pion creation, propagation, and absorption are of particular importance to the understanding of the energy loss and transport processes in relativistic heavy-ion collisions. Measurements up to the highest pion energies available at LAMPF, where the increased mean free path of the pion allows for a detailed study of its interaction over a large fraction of the nuclear volume, are imperative for understanding such reactions as multi-nucleon absorption that dominate at high density. LAMPF is the place to carry out these studies, as it provides the highest energy pion beams of all the meson factories.

The three charge states of the pion make both singleand double-charge-exchange reactions possible. Pion single charge exchange (SCX) proved an ideal tool for the discovery and characterization of the isovector monopole giant resonance. An entirely new spectroscopy of nuclear states as seen in pion single charge exchange is planned, centered around a new high-resolution, large-solid-angle detector, the Neutral Meson Spectrometer (NMS), operational in 1992. Pion double charge exchange (DCX) is the only reaction of noncomposite particles that requires participation of two nucleons. Low-energy pions have been shown to display an enhanced sensitivity to shortrange dynamics and nucleon correlations in nuclei. The world's best low-energy pion channel and spectrometer system, coupled with a new superconducting RF cavity (Scruncher), provides the data needed to distinguish between dynamical models of the nuclear wave function at short range. Both nuclear collectivity and nucleonnucleon correlations are important aspects of understanding the nucleus as a strongly interacting manybody system, as cited in the Long Range Plan.

High-resolution studies in the resonance region allow the special properties of the pion, such as the strong isospin dependence of its interaction with nucleons, to be exploited as a sensitive probe of nuclear structure. EPICS provides this capability and is unique in the world.

At yet higher energies, the pion can be used to address many of the other major topics emphasized in the Long Range Plan, such as flavor physics through the $(\pi, K)$ reaction leading to $\Lambda$-hypernuclei, nonperturbative $Q C D$ through the baryon mass spectrum, modifications of the elementary interactions within the nuclear medium owing to its relatively weak two-body interaction and long mean free path, and tests of the Standard Model through decays of the $\pi$ and $\eta$ mesons. The pion linear accelerator (PILAC) initiative at LAMPF will provide all of these new physics opportunities.

It is clear that a strong program in pion physics is now in place for the foreseeable future, certainly within the time frame of the 1989 NSAC Long Range Plan. It is also clear that the continued operation of LAMPF was a basic assumption of the Long Range Plan, central to the broadly based and balanced advances envisioned in that document. The LAMPF program will provide the understanding of processes that are of fundamental 
importance to virtually all areas of medium energy physics.

\section{Recent Accomplishments and the Ongoing Program}

Pion beams are now an established tool in the quest to understand the structure of the nucleus, the mechanisms at work in nuclear reactions, and the fundamental structure of the nucleon and the baryon resonances. Highlights of accomplishments naturally fall into three categories: chiral symmetry breaking, nuclear structure, and hadrons in the nuclear medium.

\section{Chiral Symmetry Breaking}

Chiral symmetry is an important aspect of the strong interaction, imfiying that within the hadron framework the system is unchanged under certain rotations in the limit that $\mathrm{m}_{\pi} \rightarrow 0$. Chiral perturbation theory, built on this symmetry principle, offers hope of providing a connection between low-energy phenomena and QCD. As pointed out in the Long Range Plan, considerable precision low-energy experimental data on meson interactions are required to test this relationship.

\section{Chiral Symmetry Breaking, $\pi$ N Scattering}

The availability of intense pion beams at meson factories has led to the determination of $\pi \mathrm{N}$ scattering observables with a degree of precision not previously possible. The extrapolation of these scattering amplitudes to an unphysical region determines the pion-nucleon $\sigma$ term, which can be related to the breaking of chiral symmetry in the QCD Lagrangian. This symmetry breaking involves the masses of the quarks, and may be interpreted in terms of the strange quark content of the nucleon.

The first comprehensive measurements of $\pi p$ scattering below $100 \mathrm{MeV}$ (performed at LAMPF a decade ago) disagreed with fragmentary earlier data and with predictions based upon extrapolations of established phase shifts. This controversy and interest in the $\sigma$ term inspired extensive remeasurement of these cross sections. Disagreements among the data remain, but there is an evident trend toward confirmation of the original LAMPF measurements and a clear disagreement with the accepted phase shifts derived from data heavily weighted by older energy measureisents at higher energies. It is now important to reassess the higher energy data. A 1991 LAMPF experiment measured integral cross sections at energies spanning the resonance, finding values that are significantly smaller than the established phase shift predictions. LAMPF continues to make unique contributions to this investigation: the measurement with the $\pi^{0}$ spectrometer of the cross section for charge exchange scattering, $\pi^{-} p \rightarrow \pi^{0} n$, at energies as low as 10 MeV was only possible at LAMPF.

\section{Chiral Symmetry Breaking, $\pi \pi$ Scattering}

Chiral symmetry breaking in the effective $\pi N$ Lagrangian may be characterized by a single parameter. The threshold value of the matrix element for the production of a pion in $\pi N \rightarrow \pi \pi N$ depends only upon this one symmetry-breaking parameter and other known quantities such as the $\pi \mathrm{N}$ coupling constant, within the approximations of soft pion theory. Relying on the same model assumptions, the $\pi \pi$ scattering lengths may be extracted from measurements by extrapolating the energy dependence of the total cross section to threshold. Accurate measurements at low energy are crucial.

Experiments at LAMPF, exploiting the intense beams, play an important role in these investigations. As this field has evolved, kinematically complete experiments have become a litmus test for the theoretical approximations needed to extrapolate to zero energy. Measurements involving reaction channels in which one of the outgoing pions is a $\pi^{0}$ are essential, and this capability is unique to LAMPF. An experiment designed to measure the cross section for $\pi^{+} p \rightarrow \pi^{+} \pi^{0} p$ by detecting a charged particle in coincidence with the $\pi^{0}$ will be continued in 1992. A much improved version of this experiment with the Neutral Meson Spectrometer is possible once this instrument is fully operational. The possibility exists of observing the completely neutral final state $\left(\pi^{0} \pi^{0} n\right)$. The high efficiency of the NMS and the high intensity pion beam available at LAMPF will allow data to be taken closer to threshold. Kinematically complete exclusive data on $\pi \mathrm{N} \rightarrow \pi \pi \mathrm{N}$ reactions allow a model-independent determination of the $\pi \pi$ scattering lengths and phase shifts by using the Chew-Low extrapolation method.

\section{Nuclear Structure}

The pion is the lightest strongly interacting particle. It is a probe of the nucleus that is complementary to the electron and the nucleon - each is sensitive to different aspects of nuclear structure. Experiments at LAMPF have uncovered and explored a rich variety of properties of nuclei and nuclear transitions. The strong difference between $\pi^{+}$and $\pi^{-}$scattering from neutrons and protons, at incident energies near the $P_{33}$ [or $\left.\Delta(1232)\right] \pi$-nucleon 
resonance, has been used to determine the different roles played by neutrons and protons in nuclei. Understanding the nucleus as a strongly interacting many-body system of mesons and nucleons, as called for in the Long Range Plan, is nuclear structure research. A brief list of some important results from pion-nucleus physics at LAMPF is given below.

\section{Determination of Neutron Distributions in Nuclei}

In combination, electron-nucleus and pion-nucleus scattering have been used to measure the neutron density distribution of nuclei. In the case of ${ }^{3} \mathrm{H} /{ }^{3} \mathrm{He}$, the extracted proton and neutron radius differences were found to imply a charge-symmetry-breaking effect in the mass-3 system that is consistent with the ${ }^{3} \mathrm{H} /{ }^{3} \mathrm{He}$ binding energy difference deficit (after accounting for the Coulomb energy). Analysis of elastic scattering data on the calcium isotopes has shown that the valence neutron radius is largest for $A=42$ and becomes smaller as more neutrons are added. The SCX reaction on aligned ${ }^{165}$ Ho has been used to determine the deformation of the neutron distribution.

\section{Exploration of Isospin Mixing in Nuclear States}

The direct comparison of $\pi^{+}$and $\pi^{-}$inelastic scattering has proved to be an ideal method of determining the neutron/proton or isospin character of nuclear excitation. In ${ }^{12} \mathrm{C}$ and ${ }^{16} \mathrm{O}$, strong isospin mixing was observed between stretched $4^{-}$states. The isospin mixing between the $4^{-}$states in ${ }^{12} \mathrm{C}$ is so strong that it causes the formation of nearly pure neutron and proton particlehole states. This technique has been validated by the good agreement of the extracted isospin mixing matrix element for the $1^{+}$doublet in ${ }^{12} \mathrm{C}$ with that obtained from electromagnetic measurements.

\section{Measurement of Nuclear Transition Effective Charges}

Pion inelastic scattering has determined isovector and isoscalar effective charges for transitions to low-lying nuclear states. For example, the analysis of the $2_{1}^{+}$state in 180 revealed the extent to which collective modes of excitation dilute the pure neutron nature of this transition. The result of a later analysis of the first three $2^{+}$levels in 180 was a striking confirmation of the co-existence model of Brown. Another clean example of the need for effective charges are transitions to the low-lying states of $15_{\mathrm{N}}$ and ${ }^{17} \mathrm{O}$ nuclei which are one hole or one particle removed from the doubly-magic nucleus 160 .

\section{Characterization of Stretched States}

The strong excitation of high-spin stretched states was an unanticipated feature of pion-nucleus reactions, and the discovery at LAMPF of essentially pure neutron and pure proton states in ${ }^{13} \mathrm{C}$ (shown in Fig. II-1), and other nuclei came as a complete surprise. Because of the dominance of spin-flip at momentum transfers near the peak of their form factors, these states are easily separated from the background. Such transitions have been observed throughout the p-shell, the s-d shell, and from some nuclei in the $\mathrm{f}-\mathrm{p}$ shell. In contrast to transverse electron scattering, which is sensitive only to $\Delta T=1$ transitions, the pion excites both $\Delta T=1$ and $\Delta T=0$ transitions. Thus, the comparison of spectra using these complementary probes has been used to untangle the isospin character of such excited states. The observation that quenching of the strength in $\Delta \mathrm{T}=0$ transitions (when compared with shell model predictions) is greater than that observed for $\Delta T=1$ transitions by other probes was an important result from pion scattering.

\section{Discovery of the Giant Isovector Monopole Resonance}

The $\left(\pi^{-}, \pi^{0}\right)$ single charge exchange reaction is ideally suited for discovering and characterizing the giant

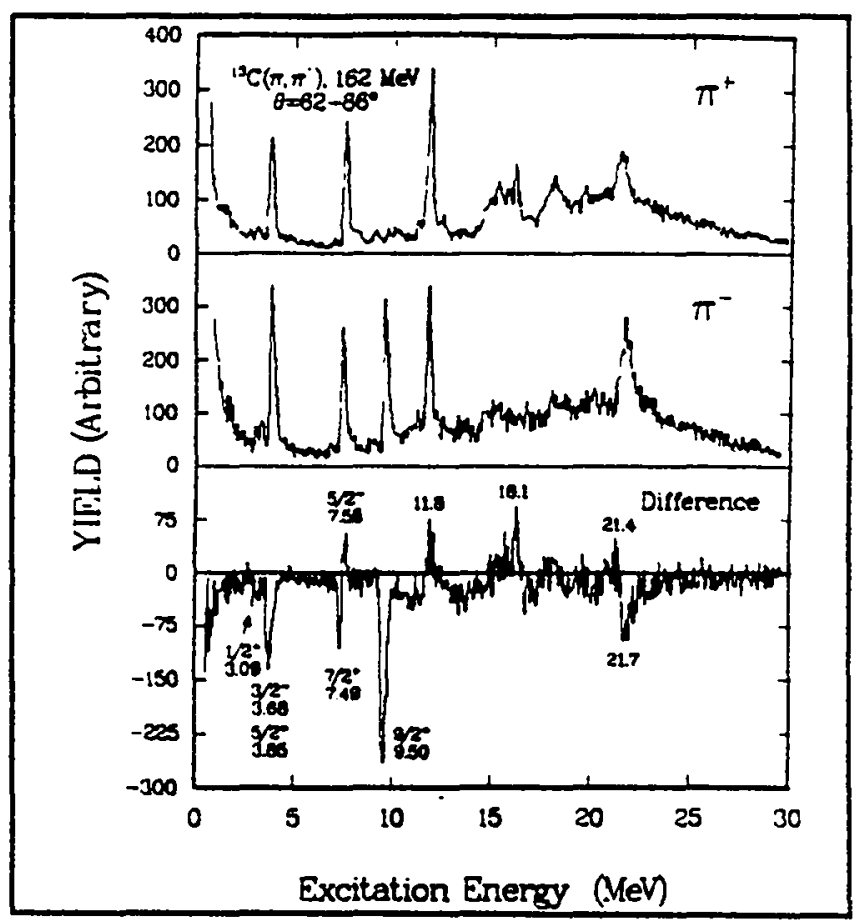

Fig. II-1. Back angle spectra for ${ }^{13} C\left(\pi^{ \pm}, \pi^{ \pm}\right)^{13} C^{\prime}$. The state at $9.5 \mathrm{MeV}$ shows the free cross section ratio of 911 . as expected for a neutron state. 
isovector monopole resonance, an important nuclear collective mode: (1) the $\pi^{0}$ single-charge-exchange reaction eliminates the possibility of the dominant $\Delta T=0$ isoscalar excitation, i.e., it acts as a filter, (2) the energy of the excited state is lowered by the Coulomb displacement energy in the reaction; and (3) the strong absorption near the $\mathrm{P}_{33}$ resonance ensures a surface peaked reaction, which is required to excite the monopole.

\section{Discovery of Double Resonances in Nuclei}

The fact that the pion occurs in three charge states has been utilized to investigate nuclear levels with exotic quantum numbers. In other reactions, these states are buried in the background. Although double analog states were known to exist in light nuclei, it was not until sufficient intensities of pions were available at LAMPF. that double isobaric analog resonances were shown to exist as narrow states in heavy nuclei. These levels provide the simplest example of double resonances as they can be constructed from the parent nucleus by applying the isospin lowering operator twice.

The pion double charge exchange reaction has also been used to isolate two phonon collective states. Both giant dipole resonances built upon the isobaric analog state (GDR $\otimes$ IAS) and giant dipole resonances built upon giant dipole resonances (GDR $\otimes$ GDR) have been observed. An example of these resonances is shown in Fig. II-2. These discoveries illustrate just how durable are such collective excitations of the nucleus.

\section{Creation of Exotic Nuclei}

The clean identification of nuclear isotopes off the line of stability gives pion DCX its power in rare isotope production. Examples of its utility include (1) the creation of ${ }^{58} \mathrm{Zn}$, a heavy $\mathrm{T}_{\mathrm{Z}}=-1$ isotope needed in various mass formulae, (2) the search for a bound state of the exotic ${ }^{9} \mathrm{He}$ isotope using the ${ }^{9} \mathrm{Be}\left(\pi^{-}, \pi^{+}\right)$reaction, and (3) the ability to measure the masses of most of the light $\mathrm{T}_{z}=2$ proton-rich nuclei near the proton drip line. The exploitation of this method of spectroscopy and mass measurements of neutron-rich nuclei is currently limited only by statistics.

Pion DCX on a ${ }^{11}$ B target has provided a unique probe of the neutron halo in the exotic nucleus ${ }^{11} \mathrm{Li}$. A radius of $\approx 5 \mathrm{fm}$ for the last two neutrons was established, which is consistent with small neutron binding; claims of radii $\geq 6 \mathrm{fm}$ are ruled out by the sensitivity of the DCX cross section.

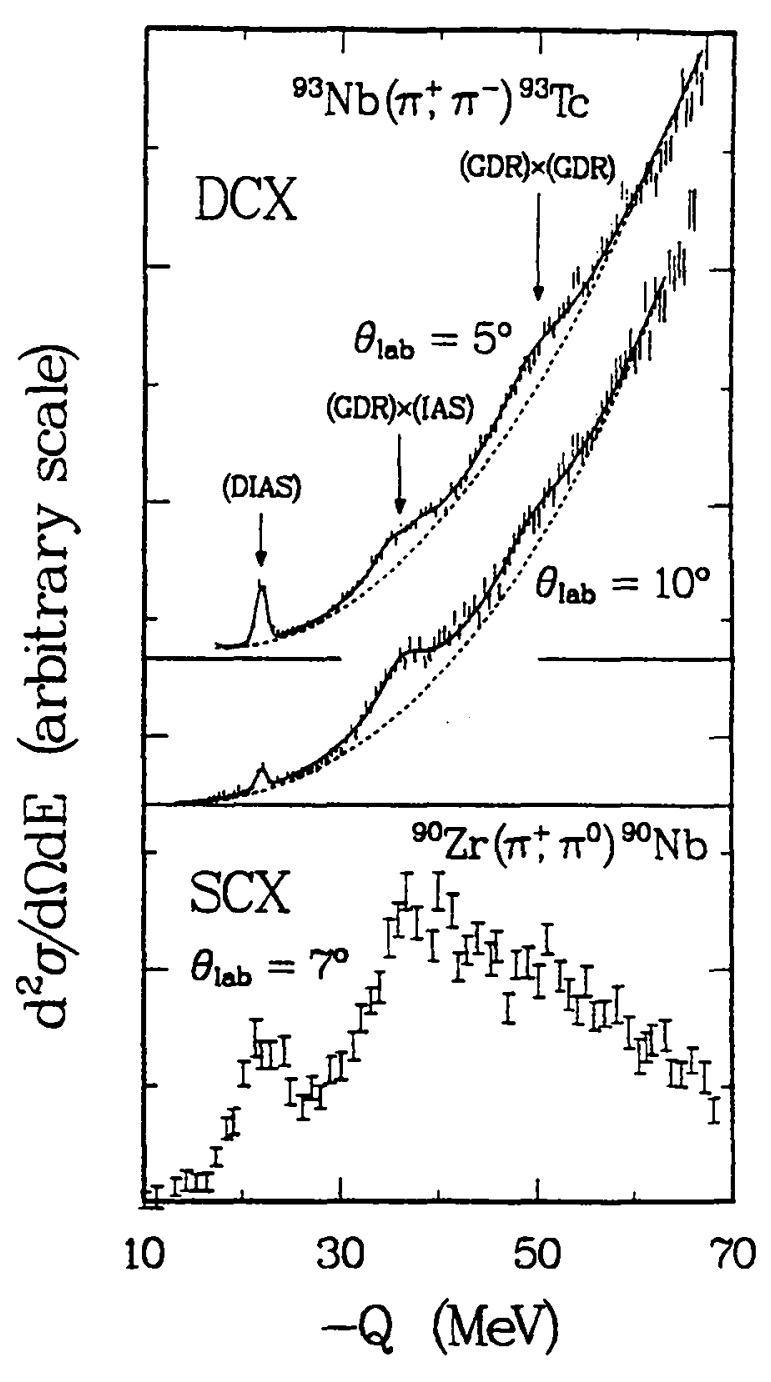

Fig. II-2. Bottom: Spectrum for ${ }^{90} \mathrm{Zr}\left(\pi^{+}, \pi^{0}\right){ }^{90} \mathrm{Nb}$ showing the isobaric-analog (IAS) and giant-dipole (GDR) states. Top: Spectra for ${ }^{93} \mathrm{Nb}\left(\pi^{+}, \pi^{-}\right)^{93} T_{c}$. Here, the double isobaric analog, the GDR built on the IAS, and the GDR built on the GDR can all be observed.

\section{Investigation of NN Correlations in Nuclei}

Low-energy (50-MeV) pion DCX on the Ca isotopes has established the sensitivity of this reaction to nucleonnucleon correlations. Analysis of the isotope measurements demonstrated that the short- and long-range components of the double isobaric analog DCX reaction could be distinguished. Predictions prior to the measurement of the relatively isotropic cross section in the case of ${ }^{48} \mathrm{Ca}$ confirmed that the seniority model 
formed a good basis for the analysis, and that the model calculations could be extended to ${ }^{54} \mathrm{Fe},{ }^{52} \mathrm{Cr}$, and ${ }^{50} \mathrm{Ti}$.

An example illustrates how nuclear states can be used as a filter to extract the desired information about the short-range interactions. A separation between long-range and short-range interactions occurs in the shell model as that model shown by de Shalit and Talmi. The long-range and short-range components of the interaction contribute differently as the number of like pairs of active particles in the nucleus is increased. In low-energy pion double charge exchange to isobaric analog states, the same formalism applies and leads to a scaling formula for the DCX amplitude:

$$
F=[n(n-1) 2]^{1 / 2}[\alpha+\beta /(n-1)],
$$

where $\alpha$ and $\beta$ are two functions of angle that are independent of the number, $n$, of valence neutrons occupying the $f_{7 / 2}$ shell. The coefficient $\alpha$ accounts for long-range interactions and the coefficient $\beta$ accounts for zero-range (or spin-dependent) interactions. This parameterization of the cross section explains a large amount of data spanning the beam energies available at LAMPF, where the $\pi N$ interaction undergoes dramatic variations.

Figure II-3 illustrates the importance of the correlations. Three calculations are compared with the data from LAMPF: the curve labeled "no correlations" shows the rapid n-dependence that would occur in the absence of shell-model and short-range correlations; the curve labeled "shell model" shows the dramatic reduction of the n-dependence due to shell-model correlations; and the curve labeled "shell model + short range" shows how the effect of shell-model correlations is damped when short-range correlations are included. Data from Weinfield et al. imply $\beta / \alpha=5$. The ${ }^{48} \mathrm{Ca} /{ }^{42} \mathrm{Ca}$ cross-section ratio is an order of magnitude smaller than would be true for uncorrelated neutrons.

\section{Hadrons in the Nuclear Medium}

Studies of the production, propagation, and absorption of pions in the nuclear medium play a major role in contemporary nuclear physics, as discussed in the Long Range Plan. The nature of pion propagation through nuclear matter has been characterized and understood in terms of $\Delta$ dynamics. An important contribution of the meson factories to the understanding of hadrons in the nuclear medium has been to provide data that clarify the nature of the $\Delta(1232)$ and its interaction in nuclei.
These studies have not only taught us about the behavior of a resonance propagating through the nuclear medium, but have also formed a basis for understanding future experiments, both those using electromagnetic probes and those that investigate the collisions of two heavy nuclei at relativistic energies. This work also provides the intellectual foundation for microscopic optical models that are being relied on heavily for the analysis of exclusive measurements involving pion scattering.

\section{Pion Absorption}

Understanding the mechanism that controls the conversion of matter into energy when a pion is absorbed in the nucleus is a primary goal of pion-nucleus physics. Additionally, virtual absorption and emission of pions play a key role whenever a pion propagates within a nucleus. Pion absorption has far-reaching implications for the interpretation of any experiment above pion production threshold (including those at CEBAF and RHIC). Consequently, pion absorption has been the subject of intense investigation at all three meson factories. Ashery and Schiffer have reviewed much of this work. There still is no consensus as to the role that multi-nucleon (more than two) processes play in pion

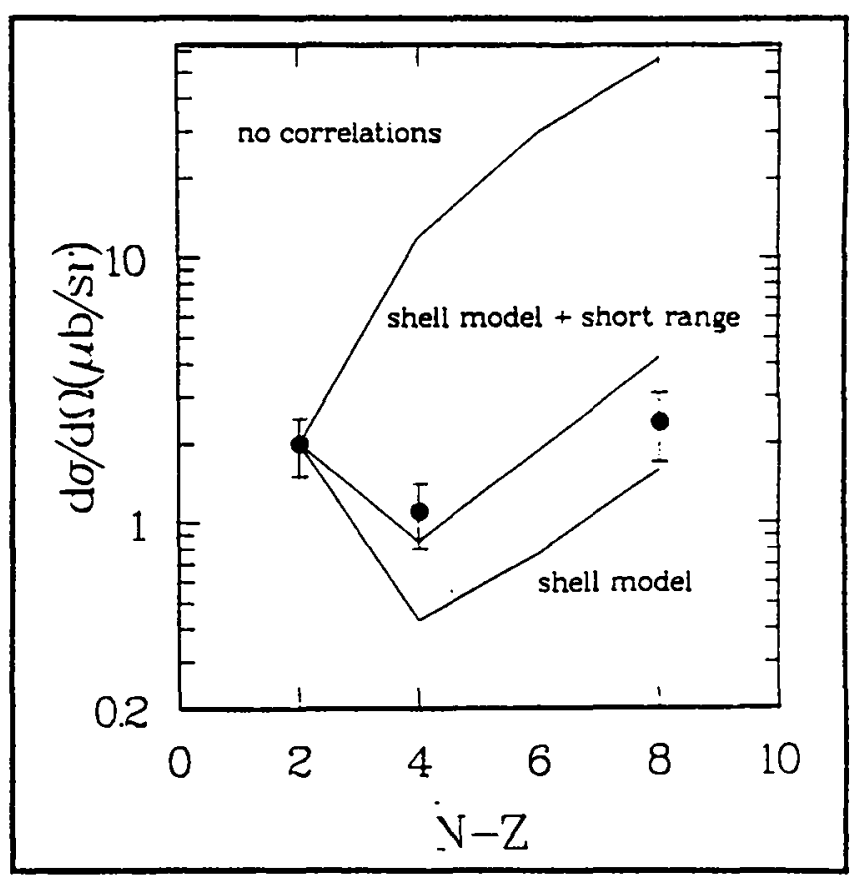

Fig. II-3. Pion double charge exchange cross sections for the calcium isutopes, $42,44,48 \mathrm{Ca}$, to the double analog states, at an incident pion energy of $35 \mathrm{MeV}$. Curves are described in the text. 
absorption on heavy nuclei. Experiments spanning the $P_{33}$ resonance have established that absorption on $T=0$ two-body pairs is important. Investigation of the ${ }^{3} \mathrm{He}\left(\pi^{+}, \mathrm{pp}\right) \mathrm{p}$ and ${ }^{3} \mathrm{He}(\pi, \mathrm{pn}) \mathrm{n}$ reactions has demonstrated the suppression of absorption involving $T=1 \mathrm{NN}$ pairs. (The $\Delta(1232)$ plays a large role in this suppression.) The additional part of the cross section has become the center of interest: absorption processes that involve three or possibly more nucleons. The first suggestion of the importance of multi-nucleon pion absorption processes was obtained from a rapidity analysis of inclusive $(\pi, p)$ data, which suggested that approximately four nucleons are involved in the absorption process on heavy nuclei. Delineation of the reaction mechanism requires kinematically complete observations, with equipment designed to detect particles over $4 \pi$ and a wide energy range.

Use of the higher energy pions available at LAMPF is imperative, to take advantage of the longer mean free path of the pion to investigate the interaction of the pion over a larger fraction of the nuclear volume. In the higher pion energy experiments performed at LAMPF (Fig. II-4), the 3-body and 4-body absorption terms have been shown to be enhanced. Characterizing such $\rho^{3}$ and $p^{4}$ terms in the absorption process is crucial to understanding the high density phenomena in heavy-ion collisions where such terms will dominate.

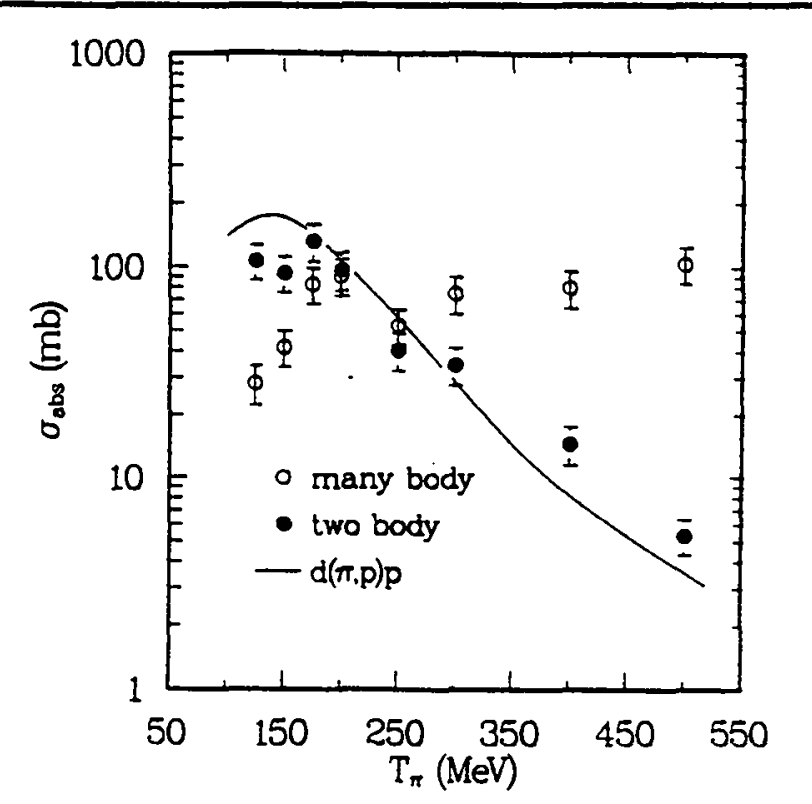

Fig. II-4. Pion absorption cross sections on ${ }^{12} \mathrm{C}$. Closed circles are two-body cross secrions (missing energies of less than $100 \mathrm{MeV}$ ). Open circles are the cross sections for large missing energy events. Solid line is the cross section for $\pi^{+}+d \rightarrow p+p$, arbitrarily normalized.

\section{Pion Propagation}

The dynamics of pion propagation in nuclear matter is described by a "dispersion relation," in which the pion dynamics is represented by the pion optical potential (or the pion self energy, as it is sometimes called). The pion optical potential is, in turn, built up of many pieces. The most important incorporate $\Delta$ propagation and interaction, short-range correlations, and nuclear structure. It has been necessary to rely on diverse experiments at the meson factories to fully model the optical potential, including pion absorption and quasi-elastic scattering, which dominate its reactive content. Recently, optical models including all of the relevant aspects of this physics have been constructed. In Fig. II-5, ${ }^{12} \mathrm{C}$ data, including recent LAMPF measurements out to $180^{\circ}$ are compared to one such model. Comparable results were obtained for higher energies up to about $500 \mathrm{MeV}$. The model includes such important fearures as realistic Hartree-Fock wave functions with full Fermi averaging, and a fully off-shell pionnucleon scattering amplitude. The delta propagation and interaction are much the same as that included in the isobar-hole model, which is complementary to the optical potential approach and briefly described next.

\section{$\Delta$ Propagation and Interactions}

Systematic studies have established that the pion, nucleon, and $\Delta(1232)$ are the important degrees of freedom in nuclei below $300 \mathrm{MeV}$. A principal motivation for studying $\Delta$-nucleus interactions is to understand the role of baryon internal structure in the strong interaction. The $\Delta$ has been found to retain its identity in the medium. Thus, a determination of the $\Delta-N$ interaction, the modification of the $\Delta$ width in the medium, etc., are needed to go beyond a naive $\Delta$-hole concept, which has worked well in certain areas, and to support quantitative conclusions from calculations that are now being carried out to explore three-body force effects in few-body systems.

Optical models such as the one on which Fig.II-5 is based, rely heavily on understanding the dynamics of the $\Delta$. In the $\Delta$-hole model, the $\Delta$ is treated as a quasiparticle, propagating and interacting in the nuclear medium. The average potential experienced by a $\Delta$ in the nucleus is well approximated by

$$
v_{\Delta}=\left(U_{0}+i W_{0}\right) \frac{\rho(r)}{\rho(0)}+\text { spin orbit , }
$$

where $\rho(r)$ is the nuclear density. Here, the potential depth, $U_{0}=-30 \mathrm{MeV}$, summarizes binding and Pauli effects, as well as dispersive corrections from absorption 


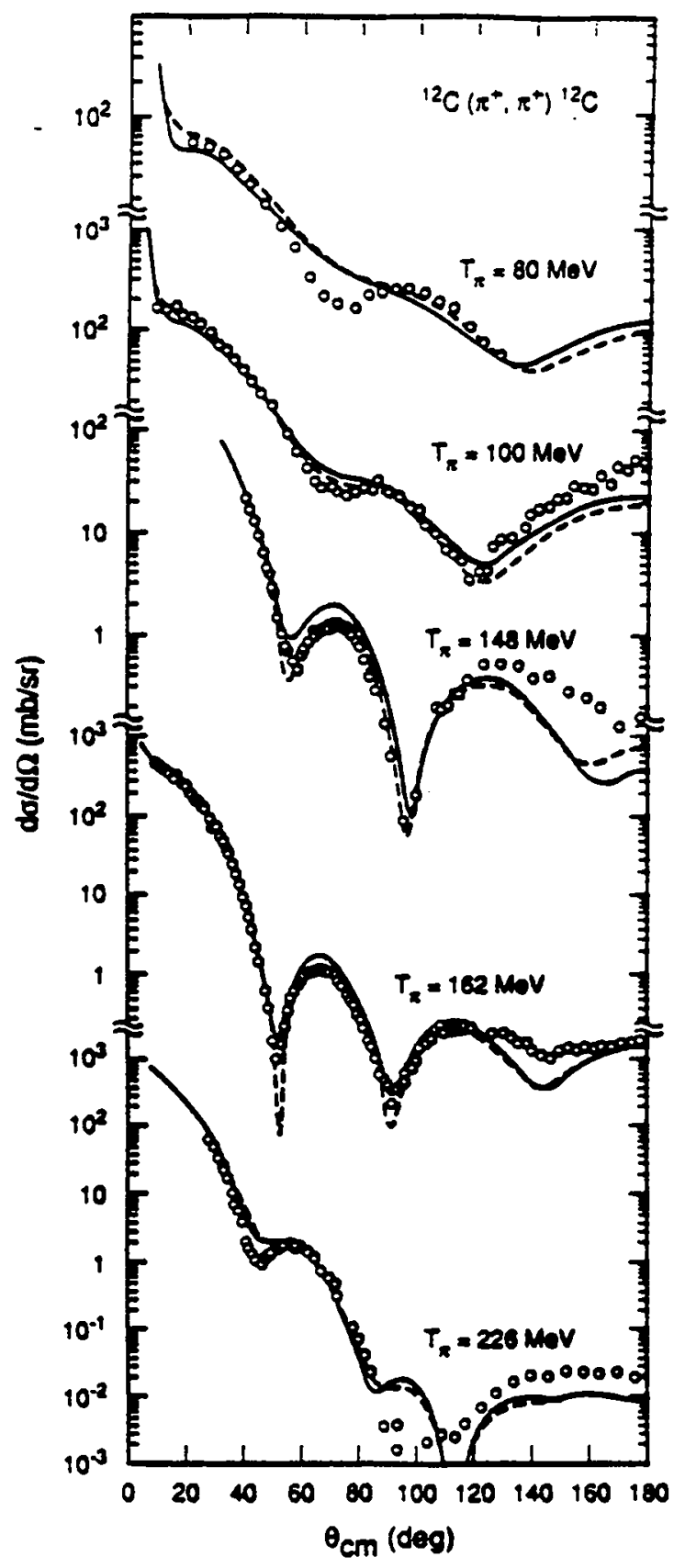

and other many-body effects. The imaginary part, $\mathrm{W}_{0}=$ $-40 \mathrm{MeV}$, includes a reduction of the $\Delta \rightarrow \pi \mathrm{N}$ width due to Pauli blocking, which is, however, ovencompensated by the presence of genuine nuclear decay channels such as $\Delta \mathbf{N} \rightarrow \mathbf{N N}$. Data from all of the meson factories have contributed to the isobar-hole model development. Agreement between different laboratories has established the integrity of the data base.

Models of pion reactions are especially important for determining the spreading of the $\Delta$ in the optical potential and isobar-hole analyses. Figure II-6 compares a microscopic model of pion two- and three-body absorption and quasi-elastic scattering to the empirically-determined $W_{0}$, used in the optical potential in Fig. II-5. The same descriptions of pion propagation and isobar dynamics provide essential input for modeling heavy-ion scattering, where hadron dynamics in high-density nuclear matter is of great importance.

The spreading interaction is also important in photonuclear reactions. For example, Fig. II-7 shows a calculation of coherent photoproduction in the isobar-hole approach. The additional damping of the resonance caused by the imaginary part of the spreading interaction in Eq. (2) is quite large. The behavior of hadrons in the

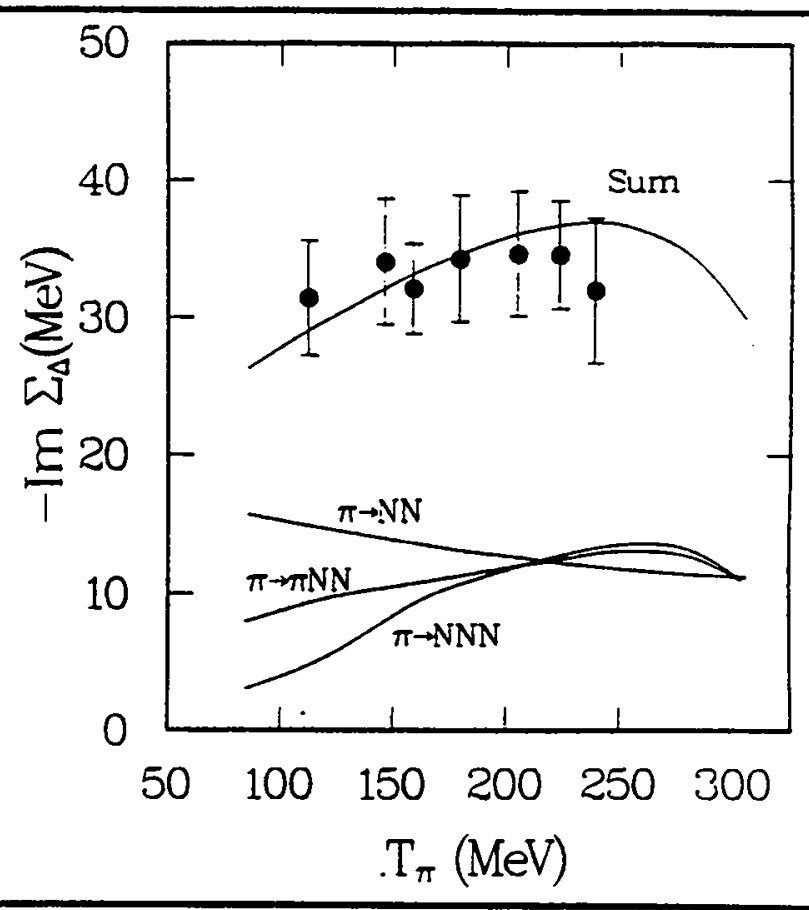

Fig. II-6. Imaginary part of $\Delta$ self-energy. $\pi \rightarrow N N$ accounts for two-body absorption; $\pi \rightarrow$ NNN for threebody absorption; and $\pi \rightarrow \pi N$ for the quasi-elastic contribution. The upper line is the sum of the three and the data points are the empirical determinction from Eq. (1) at $\rho=0.75 \rho 0$. 


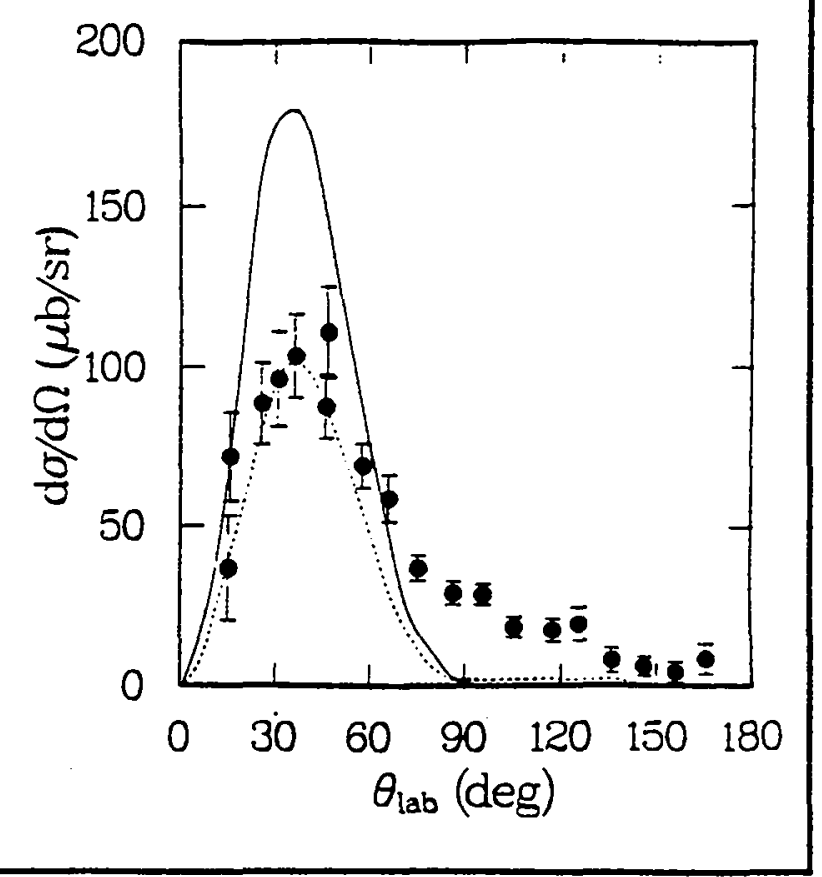

Fig. II-7. Coherent $\pi^{0}$ photoproduction on ${ }^{12} C$ at $235 \mathrm{MeV} / \mathrm{c}$. The dashed (solid) line is the calculation with (without) spreading potential.

nuclear medium, as synthesized from meson factory data, has influenced the understanding of similar phenomena encountered with electromagnetic probes.

Yet another aspect of the $\Delta$-nucleus interaction, which is accessible through pion scattering, is the Fermi liquid parameter gó $\Delta \Delta$. This parameter has been empirically determined by systematic analyses of elastic, SCX, and DCX data that have come from all the meson factories. This parameter is intimately related to shortrange correlations in the nucleus and is of about the right size to explain the difference between $\mathrm{U}_{0}$ and the corresponding quantity for nucleons. It plays an important role in the theoretical description of pion propagation in nuclear matter for calculations mentioned earlier.

\section{Pion-Nucleus Transparency}

Forward pion SCX at $50 \mathrm{MeV}$ has demonstrated the important role of the Pauli principle for nucleons in the forward propagation of pions. The phase space governing the struck nucleon's recoil is severely constrained, which significantly increases the transparency for pion propagation through the nucleus. Recent work has been concentrated below $100 \mathrm{MeV}$. Investigation at energies well above the resonance, where the $\pi \mathrm{N}$ interaction again becomes weaker, is now necessary.

\section{Inclusive $D C X$}

Near the $P_{33}$ resonance, the inclusive $\left(\pi^{-}, \pi^{+}\right)$ reaction cross sections saturate with increasing mass number. This is in contrast to the monotonic increase for the $\left(\pi^{+}, \pi^{-}\right)$reaction. The saturation is consistent with a reaction dominated by sequential SCX competing with the more probable quasi-free scattering and absorption reactions. At higher energies, other $\mathrm{DCX}$ reaction mechanisms such as meson exchange currents, $(\pi, 2 \pi)$ production followed by absorption of the extra pion, and even DCX involving an intermediate $\eta$ should play a significant role. Experiments at LAMPF are investigating the region above the $\mathrm{P}_{33}$ resonance with the existing capability.

\section{Quasi-Free Knockout Reactions}

An important part of the research at the meson factories has been the investigation of the microscopic pion and $\Delta$ interactions in the nuclear medium using quasi-free reactions. The best example is the isolation of the contribution to the coincidence $(\pi, \pi N)$ cross section in which a $\Delta$ is formed and knocks out a nucleon. An important contribution to this investigation was carried out with the $\pi^{0}$ spectrometer to study the ${ }^{16} \mathrm{O}\left(\pi^{+}, \pi^{0} \mathrm{p}\right)$ reaction. Recent work on ${ }^{4} \mathrm{He}\left(\pi^{ \pm}, \pi^{ \pm} p\right)^{3} \mathrm{H}$ has provided evidence for interference of quasi-free proton and triton knockout processes. The more complex process $(\pi, \pi N N)$ provides a means of studying the propagation of a fully off-shell intermediate pion over a tunable range of invariant mass and momentum transfer, and the interference of all three isospin amplitudes accessible to $\pi N N$ by selecting various combinations of $T_{3}(\pi)=0 \pm 1$ and $T_{3}(N)= \pm 1 / 2$ with a $T=0$ target. Comparison with (e,e'NN) experiments will provide complementary information.

\section{$\eta$ Production}

Pion-induced $\eta$ production has been investigated at LAMPF in both exclusive and inclurive reactions. Experiments on the proton determined a value for the threshold production cross section. The ${ }^{3} \mathrm{He}(\pi, \eta)^{3} \mathrm{H}$ reaction yielded the first exclusive $\eta$-production cross section on a nucleus. The inclusive cross section measurements provided an estimate of the $\eta$-nucleon reaction cross section. The goal of this work is to study the propagation and interaction of the $\eta$ in the nuclear 
medium. Further progress awaits the improved solid angle and energy resolution that will be available with the NMS spectrometer.

\section{Near-Term Program at LAMPF}

A strong physics program is presently being pursued at LAMPF. This includes investigations that will be made with new facilities, which have recently or will soon be commissioned. Specific examples include the following.

\section{The Pion-Nucleon Program}

Pursuit of chiral symmetry breaking, cited in the Long Range Plan, will continue as a priority at LAMPF, including both $\pi \mathrm{N}$ and $\pi \pi$. Measurements of integral cross sections for $\pi^{+} p$ scattering, which began in 1991, will be extended in energy, and data will be taken for $\pi^{-} p$ scattering in 1992. Measurements of $A_{y}$ in $\pi$-p scattering at low energies will

begin in 1993. The large solid angle of the NMS spectrometer, which is expected to be completed in 1993, will allow precision measurements of cross sections and spin observables in the charge exchange channel from the lowest to the highest energies available at LAMPF. Lowenergy beam intensity and the NMS will be exploited to measure the $\pi^{+} p \rightarrow \pi^{+} \pi^{0} p$ cross section.

\section{Pion Single Charge Exchange}

This program is just getting under way in its new, high-resolution, phase. The completion of the Neutral Meson Spectrometer, scheduled for its first tests in the summer of 1992 and scheduled for production running in 1993, will allow unprecedented resolution ( $300 \mathrm{keV}$ compared with $3 \mathrm{MeV}$ with the old $\pi^{0}$ spectrometer) in the detection of neutral $\pi$ 's and $\eta$ 's. An entirely new field of spectroscopy of nuclear states using pions as probes, e.g., the study of Gamow-Teller transitions, is opened by this instrument. The ability to trade resolution for solid angle makes the NMS a large solid angle device for experiments where rates are low.

\section{Pion-Nucleus Double Charge Exchange}

Pion-nucleus double-charge-exchange data have been taken over the full energy range of pion energies available at LAMPF. While yielding much new physics, these investigations are hampered by small cross sections and consequent low counting rates. At $50 \mathrm{MeV}$, counting rates for spectrometer experiments can be increased by more than a factor of five by reducing the energy spread of the beam by use of a superconducting RF-cavity, the Scruncher. The increased fluxes within a constant momentum bite now available with the Scruncher are being used to extend low-energy DCX measurements to higher mass nuclei, and specifically to isotopic sequences above the $f_{7 / 2}$ shell. This technique would be even more powerful at resonance energies where net gains of up to 10 in counting rates can be obtained over the current capabilities of EPICS for experiments that are not energy resolution limited. Implementing this scheme requires building a multicell superconducting RF cavity, the Super-Scruncher. Such plans are under way.

Improved counting rates will open a number of new areas of DCX investigations. An example is the ability to obtain data at larger momentum transfers where the role of shor range correlations is expected to be enhanced relative to longer range phenomena. Another example is the enhanced sensitivity to exotic double resonances, such as the double Gamow-Teller resonance, which can be obtained using the large spin-flip cross sections inherent at pion energies below the $P_{33}$ resonance. The improved useful beam intensities will shorten running times from days to hours for such experiments. Both correlations and collectivity are important aspects of understanding the nuclear many-body system, as specified in the Long Range Plan.

Future research will also explore the new aspects of DCX that have been uncovered. For example, the shellmodel correlations play a much more important role than had been appreciated previously; in particular, nonanalog intermediate states must play a dominant part in the reaction mechanism if the de Shalit and Talmi mechanism is the correct explanation of the dependence on the number of valence neutrons. The role of the sequential mechanism in low-energy DCX will be explored in detail using the new NMS, which has the sensitivity to resolve the low-lying single-charge-exchange routes that contribute. Mapping out intermediate-state contributions will also have implications for DCX in other energy regions and will test specific models of short-range correlations and exchange-current contributions to DCX.

\section{Quasi-Free Reactions}

The use of $\pi^{+}$and $\pi^{-}$is of extraordinary value in the case of quasi-free reactions, both in distinguishing the subsystems in the nucleus from which the pion is scattered, and in extracting the two-step process in double charge exchange with the implications for two-nucleon correlations. Current plans include a new spectrometer that will provide $200 \mathrm{msr}$ of angular acceptance and $1 \%$ 
momentum resolution for coincidence experiments with the LAS spectrometer.

\section{Pion Scattering from Polarized Nuclear Targets}

The importance of polarized target studies was discussed in the Long Range Plan. Research in this field is just beginning, as new polarized nuclear targets are being developed. Comparison of data and calculations is available in only a few cases, but the present evidence indicates that the addition of the spin degree of freedom will prove a key to our understanding of the hadronic force.

The new physics possibilities offered by polarized targets were studied initially with pion scattering on pshell targets near $\mathrm{P}_{33}$ resonance energies: ${ }^{15} \mathrm{~N}$ and ${ }^{6} \mathrm{Li}$ at PSI, ${ }^{13} \mathrm{C}$ at LAMPF and TRIUMF, and the ${ }^{13} \mathrm{C}\left(\pi^{+}, \pi^{0}\right)$ SCX reaction at LAMPF. Present $\pi$-nucleus reaction models using current nuclear wave functions have failed to explain the new polarization data in the region of the cross-section minimum. However, the data contain information about the isoscalar-spin density, in contrast to transverse electron scattering experiments that are dominated by the isovector spin part and are thus insensitive to the isoscalar part. Proton scattering is sensitive to the isoscalar spin part, but this sensitivity may be masked by the other spin-dependent terms. Thus, a successful interpretation of the experimental $\pi$-nucleus asymmetry data appears to be the best method of assessing our theoretical understanding of the isoscalar spin part of the nuclear wave function.

The striking failure of present theories in explaining the asymmetry data for the p-shell nuclei may be due to a lack of understanding of the isoscalar spin density, the reaction dynamics, or both. However, the case is different for mass-3 nuclei because there exist "benchmark" wave functions for ${ }^{3} \mathrm{H}$ and ${ }^{3} \mathrm{He}$ that are considered to be much more accurate than the wave functions of p-shell nuclei. Indeed, the recent pion scattering asymmetry data on the new high-density polarized ${ }^{3} \mathrm{He}$ target at TRUMMF were successfully reproduced using existing wave functions and reaction models.

Once the spin dependence of the $\pi$-nucleus reaction dynamics has been clarified, through scattering from the mass-3 system at resonance energies, pion scattering from heavier polarized targets allows detailed study of small components of nuclear wave functions. (The $\pi-{ }^{3} \mathrm{He}$ asymmetries become quite sensitive to the small components in the wave function in the energy region between 300 and $500 \mathrm{MeV}$.)

Because of the limited target density intrinsic to polarized gaseous targets, the high beam flux at LAMPF will be essential to the success of the experiments. The NMS will provide the increased solid angle needed to measure SCX reactions, both on ${ }^{3} \mathrm{He}$ and ${ }^{3} \mathrm{H}$. Exclusive, quasi-free scattering experiments on polarized mass-3 nuclei will supplement the elastic and charge-exchange data and are needed for investigating both the reaction dynamics, as well as the small components of the nuclear wave functions. Therefore, the current and future challenge to researchers at LAMPF, the other hadron facilities, and at the new electron facility CEBAF, is scattering and reaction experiments with pions, protons, and electrons on polarized nuclear targets. It is through comprehensive analyses of these complementary data that a deeper understanding of nuclear forces will emerge.

\section{Absorption of Pions in Nuclei}

Recent experiments aimed at identifying multinucleon processes by measuring the two-nucleon quasideuteron (QD) component directly, have been made with two detectors in the same scattering plane at angles that emphasize the QD processes. However, estimates of the total cross section for two-body absorption from these experiments require extrapolation over large regions of unmeasured phase space and are consequently modeldependent and quite uncertain. Nevertheless, the results support the conclusion that a large fraction of the pion absorption cross section is not accounted for by the $Q D$ model, and that multi-nucleon processes must be an important mechanism in pion absorption.

The relative importance of pions in heavy-ion reactions depends on the incident energies involved. At the highest energies of collision now available, $200 \mathrm{GeV}$ per nucleon, the pion multiplicity approaches 90 . This implies that, at its maximum, the density of pions is $1.2 \mathrm{fm}^{-3}$, which should be compared to a nucleon density of $0.17 \mathrm{fm}^{-3}$ for normal nuclear matter. Since, already at these energies, pions outnumber nucleons, the number of pions is expected to be $10-100$ times that of nucleons at RHIC energies. Simulations of these reactions show that the time evolution of the matter is dominated by meson rescattering and the propagation of resonances in excited nuclear matter. The creation and subsequent interaction of pions with nucleons decrease the effective mean free path, that is, they dramatically increases the "stopping." Although original predictions were that nuclei become transparent to each other at the higher energies, CERN experiments show nearly complete stopping. This is believed to be a consequence of pion-nucleon interactions.

It has been suggested that pions and other mesons provide a thermometer or gauge of the intermediate, more interesting stages of heavy-ion reactions. Because of the 
rapid creation and reabsorption of pions, this interpretation depends crucially on microscopic simulations that presently include only two-body absorption and creation. Yet we know from experiments at LAMPF and elsewhere that three-body processes must be present. Three-body mechanisms scale as the third power in the nuclear density. At the densities achieved in the high-energy heavy-ion reactions, such processes will dominate over two-body processes. A much better understanding of them will be required, and this can only be obtained by further experiments of pion absorption. Since many of the pions are created with large kinetic energy, experiments at energies above the $P_{33}$ resonance will be required. This can best be done at LAMPF. Numerical simulations of these heavy-ion reactions indicate sensitivity not only to pions, but also to intermediate $\eta$ and $\rho$ mesons. The interaction of these mesons with nucleons is very poorly understood and can only be investigated at the higher energies at facilities such as PILAC.

To explore in more detail the absorption mechanism, events that do not appear to be due to two-body absorption have recently been examined more closely using the BGO ball at LAMPF. In contrast to the coplanar two-proton events described above, the remaining absorption data show little structure and a weak angular dependence. An analysis similar to that used by McKeown et al. to find the velocity of the moving frame from which these particles are emitted, leads to the suggestion that many nucleons are involved in the absorption process.

Progress in understanding pion absorption is likely to require measurements of the emitted neutrons that appear to account for much of the missing energy even in $\pi^{+}$ absorption. In order to be successful, large solid-angle coverage is needed to track the correlations between emitted neutrons. The low detection efficiencies of conventional neutron detectors makes this a challenging experimental problem. One technically feasible scheme for detecting these neutrons may be the use of the same fast, high-density inorganic scintillator CsI(pure) that is being developed for the NMS. Initial studies of this technique are planned for the summer of 1992 . If it is successful, the NMS, operated in coincidence with the BGO ball, may provide the instrumentation needed for furthering our understanding of the reaction mechanisms underlying pion absorption.

\section{Exotic Nuclei}

Pion reactions have been used to sudy exotic nuclei. In particular, the $\left(\pi^{+}, \pi^{-}\right)$reaction has been used to measure nuclear masses near the proton drip line and the $\left(\pi^{-}, \pi^{+}\right)$and $\left(\pi^{-}, p\right)$ reactions have been used to search for neutron-rich nuclei. Many interesting cases have yet to be explored.

Recently, an exciting new technique for populating light, neutron-rich nuclei has been discovered at the Leningrad Nuclear Physics Institute. Here, $\pi^{-1} s$ are stopped in a target and heavy charged fragments are detected in back-to-back silicon telescope detectors. The missing mass of the residual nucleus can be reconstructed and this spectrum can be examined for evidence of bound or quasi-bound states in the threshold region. Even with low statistics data, obtained with beam fluxes in the $10^{4}$ range, exciting new discoveries have been made. A collaboration between Northwestern University and Moscow Engineering and Physics Institute has been formed to move this apparatus to LAMPF in order to take advantage of the much higher beam fluxes at LAMPF. This should permit much better energy resolution and greatly extend the range of applicability of this technique.

\section{Summary}

LAMPF plays a unique role in the nation's hadron physics research program. The multifaceted pion physics program, implicit in the 1989 NSAC Long Range Plan, is creative, is broadly based, is a dominant element in strong interaction physics research, and is aimed at issues that are of fundamental importance to virtually all areas of medium-energy physics.

The high-intensity beams, the unique instrumentation, and the high energy available at LAMPF are a world resource that is focused on problems that impact areas from particle aspects of hadrons to meson creation and annihilation in heavy-ion collisions. The physics is timely, ranging from chiral symmetry breaking in the $\pi \mathrm{N}$ and $\pi \pi$ systems, to nuclear structure questions addressed by the unique $\pi^{0}$ detection capability of LAMPF and the Scruncher technology pioneered at LAMPF, to precision studies utilizing enhanced polarized target capabilities, to pion absorption and propagation investigations that address the fundamental question of conversion of mass to energy, as well as more practical questions concerning the interpretation of data from electromagnetic interactions with nuclei at energies above the pion threshold and relativistic and ultra-relativistic heavy-ion collisions.

\section{Nucleon Probes}

\section{Accomplishments and Opportunities}

Physics with the primary polarized proton beam at LAMPF has been one of the most productive areas of research in medium energy nuclear physics over the past 
15 years. High precision proton-nucleus elastic scattering data from the High Resolution Spectrometer (HRS) motivated a concerted theoretical effort during the $1980 \mathrm{~s}$ in which the fundamental nucleon-nucleon (NN) interaction in the nuclear medium was studied, nuclear matter distributions in finite nuclei were obtained with unprecedented accuracy, and relativistic and nonrelativistic forms for the nucleon propagator were examined in detail. The impact on many-body nuclear physics has been enormous.

The development of focal-plane polarimetry and highresolution neutron polarimetry proved to be the crucial step in the investigation of spin degrees of freedom in nuclei. The 1989 Long Range Plan details the importance of the separation of spin-independent and spin-dependent excitation modes in nuclei, as well as the decomposition of the spin response into its longitudinal and transverse components. Such decomposition provides a direct measure of pi- and rho-meson collectivity in the nucleus. Focal plane polarimeters are now standard equipment at hadron facilities around the world, and they are rapidly becoming vital tools in the most interesting experiments at the new generation of $\mathrm{CW}$ electron machines. The fundamental NN elastic scattering data needed for understanding the electron-hadron coincidence experiments that these machines make possible have been measured with unprecedented precision at LAMPF.

New instruments are now in place to carry forward detailed and unprecedented studies of the nuclear spin response. In 1990, a new Optically Pumped Polarized Ion Source (OPPIS) with full spin precession capability began to produce the high intensity beams needed to perform difficult neutron experiments with reasonable count rates. A Neutron Time-of-Flight (NTOF) facility has been recently commissioned for high resolution polarizationtransfer measurements of $(p, n)$ reactions at high energy. A Medium Resolution Spectrometer (MRS) for (n,p) and $(p, p)$ continuum studies is in the commissioning phase. The MRS will also prove useful as a second arm in $(p, 2 p)$ and delta decay studies. Simple, flexible, and reliable polarized nuclear targets have been developed.

A strong physics program, with highlights detailed below, is in place. This program is responsive to the goals of the Long Range Plan concerning modification of fundamental properties of hadrons in the nuclen medium, separating and understanding the components of the nuclear response over the quasi-elastic region up through excitations of the delta, and fncmulating the appropriate scattering theories for complex nuclear systems. The physics here is highly complementary to the physics to be studied at Bates and CEBAF. A precise understanding of $\left(e, e^{\prime} p\right)$ in the quasi-elastic region, for example, is needed in order to know how the nucleon's properties are modified in nuclei. This can be achieved only after the corresponding response functions measured in proton scattering are understood. The nucleons measured in coincidence experiments at CEBAF generally fall into the LAMPF energy regime; an understanding of reaction dynamics in the LAMPF energy range will therefore be vital for future studies at CEBAF.

\section{The Spin-Longitudinal and Spin-Transverse Response of the Nucleus}

Important comparisons of $\pi$ and $p$ collectivity in the nuclear response are being made at LAMPF. At momentum transfers of about $1.5 \mathrm{fm}^{-1}$, the particle-hole interaction in the spin-longitudinal $(\sigma \cdot q)$ channel is expected to become moderately attractive, while the corresponding interaction in the spin-transverse $(\sigma \times q)$ channel remains weakly repulsive. It is this feature of essentially all theoretical models that led to early predictions of precritical pion condensation and then to the predicted enhancement in the quasi-elastic region of the spin-longitudinal response compared to the spin-transverse response. This enhancement is very sensitive to the nature of the probing reaction - it cannot be measured with a spin-transverse probe such as electron scattering.

The first LAMPF results, which appeared around the same time as the first EMC results, showed no evidence for a relative enhancement of the pionic response as probed by the (p,p) reaction. These results are discussed at length in the Long Range Plan. The (,$\left.p^{\prime}\right)$ longitudinal/transverse separation was made via focalplane polarimetry at the HRS. The results challenged the pionic enhancement theories, as well as theories on the pionic origin of the EMC effect. But the interpretation of the results was also controversial, primarily because $(p, p)$ cannot separate the isovector $(\Delta T=1)$ response, where the effect was predicted, from the isoscalar $(\Delta T=0)$ response, where no enhancement is expected. However, recent data from NTOF using the $(p, n)$ reaction, which selects only the $\Delta T=1$ response, also show no relative enhancement and suggest a fundamental problem in nuclear theory: where are the pionic correlations in nuclei? The attempt to answer this question provides a prime focus for the Nucleon Physics Laboratory at LAMPF.

The new data from NTOF consist of a complete set of polarization transfer observables for quasi-free $(p, n)$ reactions on ${ }^{2} \mathrm{H}, \mathrm{C}$, and $\mathrm{Ca}$ at a momentum transfer $\mathrm{q}$ of $1.72 \mathrm{fm}^{-1}$ and a bombarding energy of $495 \mathrm{MeV}$. Measurements were made with all three polarization states of the incident beam, and all polarization components of the outgoing neutrons were determined. The data for $\mathrm{Ca}$ 
are shown in Fig. II-8. The ratio of $R_{L}$ to $R_{T}$, the spinlongitudinal and spin-transverse responses, is plotted as a function of projectile energy loss. Each response is determined from a linear combination of polarization transfer observables. The data are not only consistent with the lack of longitudinal enhancement deduced from the previous $\left(p, p^{\prime}\right)$ data, but also reveal some apparent transverse enhancement at high energy loss. The theoretical curves shown (by Ichimura and Kawahigashi) are in reasonable agreement with the data only if pionic correlations are omitted. Jochen Wambach recently said that: "It looks as if the pion is completely absent, which is very hard to understand theoretically. Maybe there is some complication in the reaction mechanism, which we cannot exclude at present. The LAMPF data remain a challenge to theory. They relate to pionic interpretations of the EMC and Drell-Yan data, pion coherence effects in heavy-ion collisions, as well as properties of vector mesons and baryons in the nuclear medium."

These $(p, n)$ and $\left(p, p^{\prime}\right)$ data presently exist at only one beam energy and momentum transfer. The experiments are time consuming, but it is clear that some exploration of the momentum-transfer dependence will be necessary to clarify the role of the underlying particle-hole interaction and to assess the correctness of the reaction model used to interpret the data. A basic assumption of this model is that the quasi-free peak corresponds entirely to single-step reactions on bound nucleons, an assumption that can be investigated with coincidence measurements of knockedout particles. This question - Is the quasi-free peak

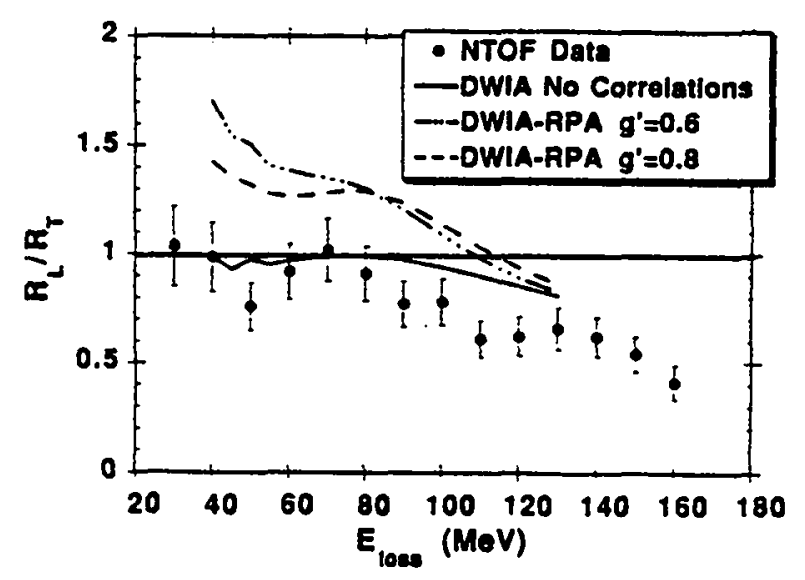

Fig. II-8. Ratio of the longitudinal and transverse spin responses for $\mathrm{Ca}(p, n)$ at $495 \mathrm{MeV}$ and $18^{\circ}(q=$ $1.72 \mathrm{fm}^{-1}$ ). The solid, dashed and dotted curves represent recent calculations by Ichimura and Kawahigashi. Best agreement with the data is obtained when correlations in the longitudinal (pionic) response are omitted. essentially all quasi-free? - can be addressed with the (p,2p) reaction using combinations of proton spectrometers or a proton spectrometer and large solid angle scintillators, but eventually the $(p, n p)$ channel must also be studied.

\section{Pionic Correlations and the Delta Resonance}

Some evidence for pionic correlations in the excitation of the delta resonance in nuclei has been observed in charge-exchange cross section measurements. It is important to follow up these initial measurements in order to understand the relationship to the properties of the nuclear response in the quasi-free nucleon sector. A thorough exploration of these basic nuclear responses will be important for later studies of nuclear delta decay that are at the top of the agenda for $\mathrm{CW}$ electron machines. The present evidence for pionic correlations in the delta region is based entirely on theoretical interpretations of cross section measurements. An example of a calculation by Udagawa, Hong, and Osterfeld is shown in Fig. II-9, where it is compared to ${ }^{12} \mathrm{C}(\mathrm{p}, \mathrm{n})$ data from LAMPF. The reaction model employed for this calculation (an example is shown in Fig. II-10) also makes clear predictions about how the spin longitudinal and transverse responses should

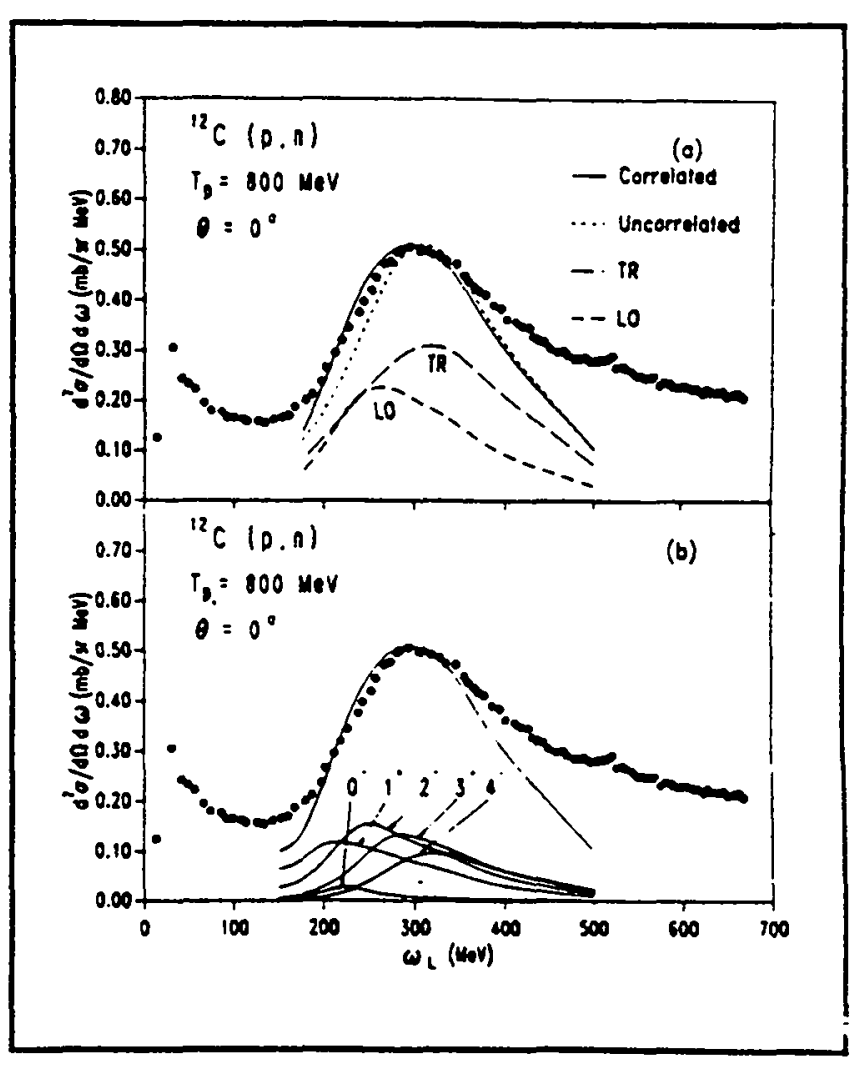

Fig. II-9. Data and calculations for the delta region of excitation in the ${ }^{12} \mathrm{C}(p, n)$ reaction at $800 \mathrm{MeV}$. The calculations are by Udagawa. Hong, and Osterfeld. 


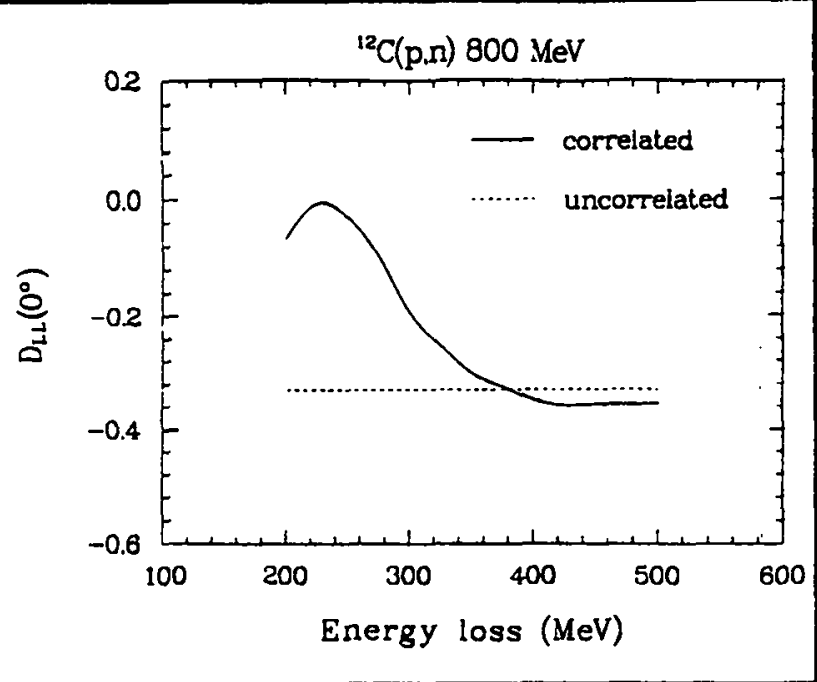

Fig. II-10. The polarization transfer coefficient $D_{L L}$ for the delta region of excitation in ${ }^{12} C(p, n)$. This calculation by Udagawa et al. predicts a dramatic signature for pionic correlations.

be manifested in the polarization transfer observables. As in the quasi-elastic region, only LAMPF can do the requisite measurements. These polarization transfer measurements have been given the highest priority.

The position of the quasi-free delta peak observed in $(p, n)$ and $\left({ }^{3} \mathrm{He}, \mathrm{t}\right)$ reactions on nuclear targets is strongly shifted toward lower energy loss compared to its position in free NN scattering. While some of this shift is kinematical in origin, the recent theoretical analysis of these data by Udagawa et al. suggests that about $30 \mathrm{MeV}$ of the shift (out of $70 \mathrm{MeV}$ ) is due to delta-particle nucleon-hole correlations in the spin-longiudinal channel. Similar theoretical results have been obtained by the Lyon group. In contrast to the hadron probes, little shift is seen in experiments with electrons, which are sensitive only to the spin-transverse response.

The relative importance of spin-longitudinal and spintransverse correlations in finite nuclei is an important issue at the heart of studies with both electron and hadron probes. The data from both types of probe and their interpretation are highly complementary; delta decay in nuclei following electro-excitation will not be understood without the hadron data.

The decay of the delta following $800-\mathrm{MeV} /$ nucleon charge exchange reactions has been reported recently by a KEK/Rutgers collaboration and by a Saclay/NBI group. The energy of the delta peak is found to be strongly dependent on the decay mode. Coincidence between the projectile and the standard free-decay products $\mathrm{p}$ and $\pi^{+}$ yields the energy corresponding to excitation of a free nucleon. However, with one pion or two protons in coincidence, strong shifts are observed similar to those seen in the inclusive spectrum.

The energy shifts observed in the decay studies are reasonably well predicted in the work of Udagawa et al. However, the cross section studies simply provide a measure of the sum of the different spin channels. The observed energy shifts alone do not provide definitive evidence for correlations in any particular channel. Polarization transfer measurements can provide the additional necessary selectivity. Some initial measu:ements of polarization transfer to the delta region will soon be carried out at NTOF. It is likely that these measurements will have as much impact on our understanding of the delta region as the first polarization transfer measurements had for the quasi-free region.

The role of pionic correlations is expected to be strong only in the single-pion decay mode where no proton leaves the nucleus; these pions are supposed to be coherent, most of which leave the target nucleus in its ground state. Neutron energy resolution in the decay experiments was not nearly good enough to separate out final states. Such resolution can be easily obtained with the NTOF facility at LAMPF, however. Even lowresolution measurements of the neutron polarization in coincidence with pions should yield much stronger evidence for correlations than neutron measurements alone. A successful description of the results from these experiments and the first decay experiments with electrons will be a giant step forward in understanding the role of the delta in the nucleus. This is an important goal of the Long Range Plan and one that is elementary in the advance toward understanding the role of heavier baryons in the nucleus.

\section{Medium Modification of Hadrons in the Nucleus}

A major component of the Long Range Plan is the understanding of the modification of the properties of the nucleon in the nucleus. Indeed, this is a major motivation for the upgrades of existing electron machines and for the construction of CEBAF. There are theoretical and experimental reasons to suggest that the properties of nucleons inside nuclei are indeed different from their free space values. The theories and consequently the interpretation of experiments in this area are highly controversial. This is true for both electron and hadron probes. Careful experiments must be done, and the same problem attacked with a number of different probes in the attempt to reach unambiguous conclusions.

The contribution of LAMPF is crucial. In the study of the delta, the relevant issue is clear. Is the delta mass 
modified inside nuclei? In the quasi-elastic region, the apparent lack of spin-longitudinal correlations may be interpreted as one indication of a medium modification of the tho meson due to an effective increase in the pion mass inside nuclei. This has been receiving considerable theoretical attention of late and would have interesting implications for $\mathrm{e}^{+} e^{-}$yields in relativistic heavy-ion collisions and for hadron form factors in dense hot nuclear matter.

Gerry Brown and his coworkers have suggested that the masses of scalar and vector mesons and nucleons obey a universal scaling with nucleon density $\rho$, of the form $\mathrm{m}^{*} / \mathrm{m}-1-\lambda \rho / 2 \rho_{0}$, where $\rho_{0}$ is the central nuclear density and the scaling constant $\lambda$ is expected to be in the range $0.2-0.4$. Such scaling would have many consequences, including an enhancement of the spin-orbit force and a reduction of the tensor interaction in the nuclear medium. Experimental studies in the intermediate energy range at LAMPF and other accelerators have yielded a firm knowledge of the $T=1$ NN phase shifts from which scattering amplitudes are derived. These scattering amplitudes are then used in nuclear reaction calculations which, in favorable circumstances, test the validity of the assumption that these amplitudes remain the same inside nuclei. By measuring spin observables for inelastic proton excitation of relatively simple nuclear configurations with well known quantum numbers, different components of the NN force inside nuclei can be determined.

Very few cases have yet been studied well enough to be convincing, but there are clear indications of significant deviations from the free values of the tensor and spin orbit forces. Studies of other transitions are needed in order to substantiate the case. A very promising way to look for such effects is via the exclusive $(p, 2 p)$ reaction to specific final states, where the density dependence can be explored in some detail. Such studies are just beginning at LAMPF, and may be greatly aided by a proposed move of the MRS to the HRS dome.

A good example of the sensitivity of polarization transfer measurements to medium modification of the interaction is provided by the study of $\mathrm{O}^{+} \rightarrow 1^{+}$transitions at zero degrees. For this type of transition, the transverse polarization transfer coefficient $\mathrm{D}_{\mathrm{NN}}\left(0^{\circ}\right)$ can be directly related to the strength of the tensor exchange amplitude. Measurements of this observable for $\mathrm{1}^{+}$transitions to discrete nuclear levels can be compared either to $2 \mathrm{H}(\mathbf{p}, \mathbf{n})$ data, which very nearly represent free scattering, or to phase-shift predictions, which are derived from freescattering. Initial data for a $1+{ }^{14} \mathrm{C}(\mathrm{p}, \mathrm{n})$ transition are shown in Fig. II-11, along with data for the ${ }^{2} \mathrm{H}(\mathrm{p}, \mathrm{n})$ reaction. Comparison of the two reactions indicates that the effective tensor exchange amplitude is systematically smaller than the free value for energies up to at least $500 \mathrm{MeV}$. It will be important to explore this observable for other nuclei to be sure this is not a poorly understood effect of nuclear structure, and to extend the measurements to higher energies.

\section{Elastic Scattering and Scattering Theory}

During the past 10 years, analyses of the LAMPFHRS proton-nucleus elastic scattering data have led to the development of sophisticated scattering models based on either the nonrelativistic many-body Schrodinger equation or the Dirac equation and relativistic quantum mean field theory. The nonrelativistic descriptions of nucleonnucleus scattering are based on approximate solutions of the many-body Schridinger equation for the $(A+1)$-body system. Most often these solutions utilize an optical potential formulation as given either by Watson or by Kerman, McManus, and Thaler. The extensive LAMPFHRS data base has driven efforts to improve the application of the scattering formalisms. Nonrelativistic optical potentials now account for the full off-shell dependence of the NN t-matrix, the full one-body nuclear density matrix, some nuclear medium effects, target nucleon two-body correlations, and important electromagnetic spin-orbit effects.

The development of the relativistic approaches based on the Dirac equation is a direct result of efforts to explain

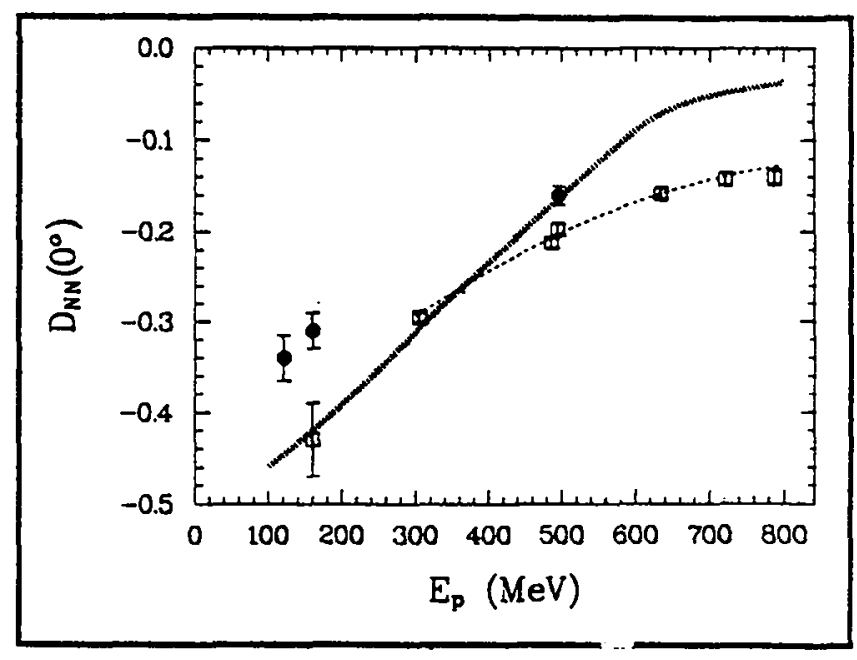

Fig. II-11. Transverse polarization transfer at zero degrees for $1^{+}$transitions. For this type of reaction, a purely central interaction would result in a value of $D_{N N}\left(0^{\circ}\right)=$ -113 . The open boxes represent ${ }^{2} H(p, n)$, the solid circles represent ${ }^{14} C(p, n)$. The broad hashed line is based on the phase-shift amplitudes of Andt, the dashed line is based on the phase-shift amplitudes of Bugg. 
the structure in the LAMPF-HRS spin observable data. In the relativistic approaches, the collision is described in terms of a Dirac proton interacting with an optical potential that is obtained from a Lorentz invariant NN amplitude and nuclear densities from relativistic mean field theory. The first relativistic microscopic proton-nucleus scattering calculation was the relativistic impulse approximation (RIA) - Dirac equation model. In this approach, a local form for the relativistic $\mathrm{NN}$ t-matrix was assumed, which included Lorentz scalar, vector, tensor, axial-vector and pseudoscalar terms. Tjon and Wallace calculated a completely general Lorentz invariant $N N$ amplitude based on covariant meson exchange theory. Transitions between positive and negative energy states are determined by the meson couplings.

In the early 1980s, the relativistic models gave vastly superior descriptions of the data. As improvements were made in the nonrelativistic models, the corresponding fits to the data have also gotten better. At present the best available nonrelativistic and relativistic model predictions are both in good qualitative agreement with existing data. However, significant differences remain in certain regimes, notably the small-angle nuclear-Coulomb interference region. An example of the differences between the two models for small angle scattering from $\mathrm{Pb}$ is presented in Fig. II-12. High precision measurements at these small angles with the HRS will provide an incisive test of current models. While the question of the importance of relativistic dynamics in nuclear systems remains unanswered, the impact of this extensive theoretical work is clear. As a result of the efforts to understand the LAMPF-HRS proton-nucleus elastic scattering data,

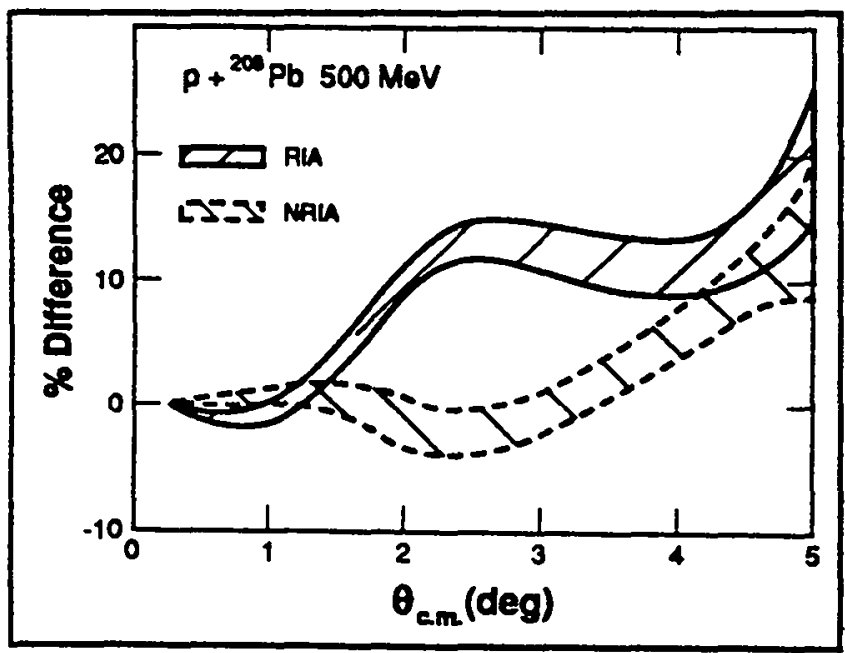

Fig. II-12. Difference between nuclear and Coulomb elastic scattering cross section in the small angle region for relativistic (RIA) and nonrelativistic (NRIA) reaction models. completely new approaches to solving the nuclear manybody problem have been developed, the traditional nonrelativistic nuclear many-body theories have been pushed to unprecedented levels, and many young theorists have joined the ranks of those trying to develop a practical relativistic quantum field theory description of nuclear phenomena.

Most of the experimental and theoretical work done so far relates to just two components of the NN force, the isoscalar non-spin-flip terms. The remaining eight components of the NN force are also interesting, as they can excite different states in the nucleus and are affected differently by the many-body environment. Studies of these other components of the NN interaction and nuclear responses are just beginning. The research programs at the MRS and NTOF are directed toward these goals. The development of convenient polarized nuclear targets provides a new way of sudying specific pieces of the NN interaction.

Elastic scattering of polarized protons from polarized nuclei at medium energies provides a highly selective method for studying the spin-flip components of the NN effective interaction, aspects of nuclear structure associated with the valence nucleons, and possible relativistic effects associated with the bound nucleon. Initial polarized-target data have been obtained for $p+{ }^{13} \mathrm{C}$ elastic scattering at $497.5 \mathrm{MeV}$ by $\mathrm{G}$. W. Hoffmann et al. These data include the target analyzing power $A_{\text {ooon }}$ and the spin correlation parameter $A_{\text {oonn }}$. Relativistic and nonrelativistic distorted wave Born approximation calculations describe the general features of the data but fail to explain some details. A new state-of-the-art polarized ${ }^{13} \mathrm{C}$ target will be used to check the first results. New experiments can then proceed to other targets.

\section{The Nucleon-Nucleon Program at LAMPF}

The LAMPF nucleon-nucleon (NN) program is essential for CEBAF, and forms the foundation of our understanding of the nucleus. Most of the approved experiments for the CEBAF HRS require precise knowledge of the NN amplitudes in the LAMPF energy range. This range is dominated by delta production, and coupling to the delta (and other inelastic channels) produces dramatic structure in several channels.

Recent experiments at LAMPF have provided a complete set of spin-dependent data for both isospin- 0 and isospin-1 elastic scattering. Examples of the new measurements are shown in Fig. II-13. The new data have a statistical weight at least one hundred times better than that achieved in previous measurements. This improvement was in part made possible by the high-intensity 


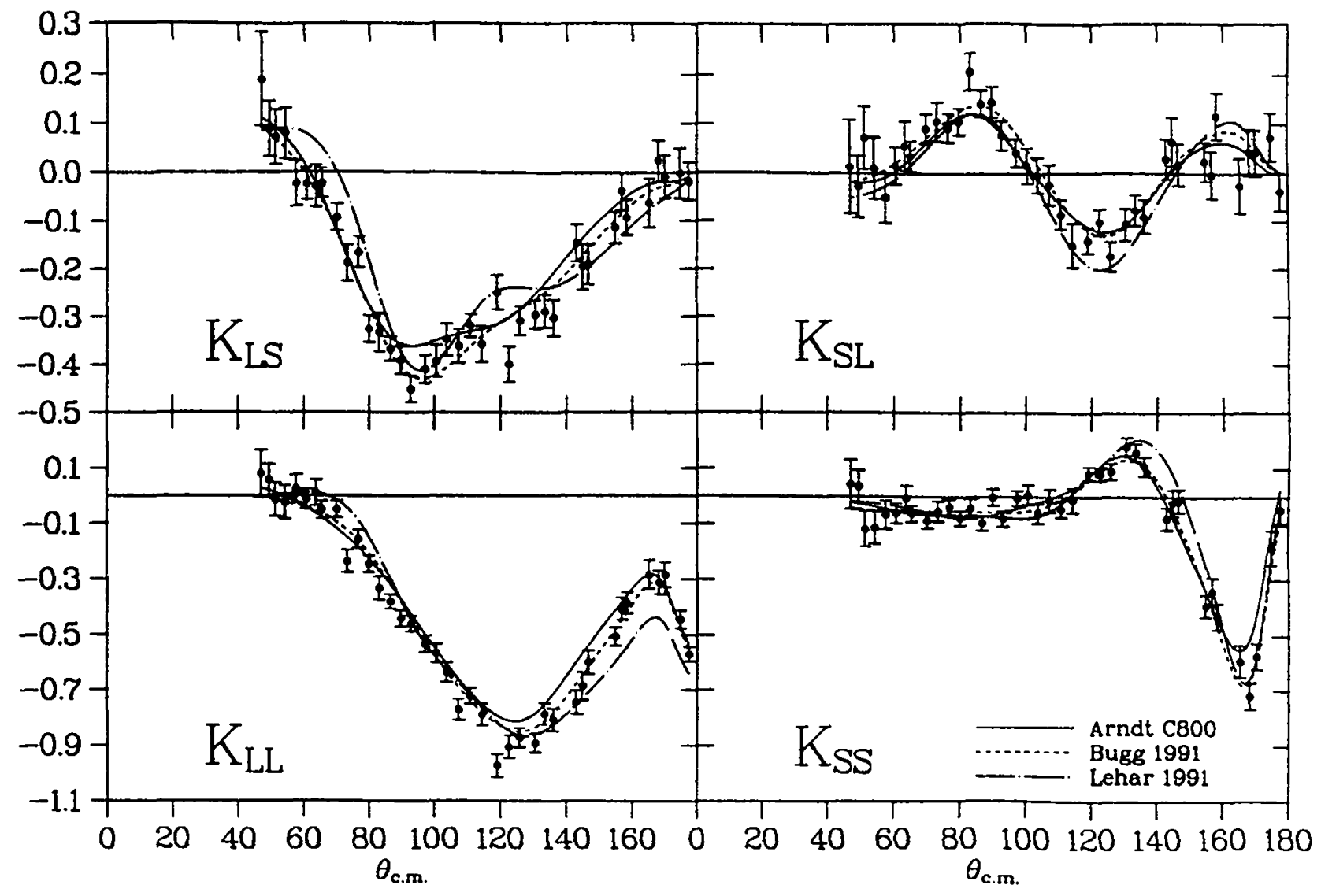

Fig. II-13. Polarization transfer observables for np scattering at $788 \mathrm{MeV}$. The data are compared to three recent phase-shift analyses.

beams provided by the new polarized ion source, and is illustrative of the new experimental capabilities now in place.

The new data have led to stable over-determined phase-shift solutions for energies up to $800 \mathrm{MeV}$. Nevertheless, significant discrepancies persist among the solutions produced by the four phase-shift-analysis (PSA) groups in the USA, UK, Paris-Geneva, and Japan (see Fig. II-13). These discrepancies are primarily a result of uncertainties in the inelasticities, which are apportioned very differently among the available channels by the four PSA groups.

Recent measurements of $\mathrm{pp} \rightarrow \mathrm{pn} \pi$ at LAMPF resulted in a partial-wave analysis for the isospin-1 inelastic process. A major program of isospin-0 inelastic measurements scheduled for 1993-1995 should complete the determination of the NN phase-shifts and amplizudes at LAMPF.
The possibility of resonance-like structure in the isospin-0 NN channels is suggested by recent measurements. Since isospin-0 NN cannot couple to a delta, such structure would indicate either coupling to a pair of deltas (below threshold) or some exotic process. Results from the isospin- 0 inelastic measurements scheduled for 1993-1995 should clarify these questions.

An important part of the LAMPF NN program has been and continues to be the measurement of precise calibration standards that are essential for nuclear physics. Proton puiarizations worldwide are precisely calibrated with reference to the $1 \%$ measurements of $\mathrm{pp}$ and $\mathrm{pC}$ analyzing powers at LAMPF. These calibrations are the foundations for polarized proton measurements planned at CEBAF. Preliminary results from the LAMPF measurement of the pp elastic cross section are shown in Fig. II-14, in the form of the ratio to the phase-shift solution of Bugg et al. The accuracy and precision of 


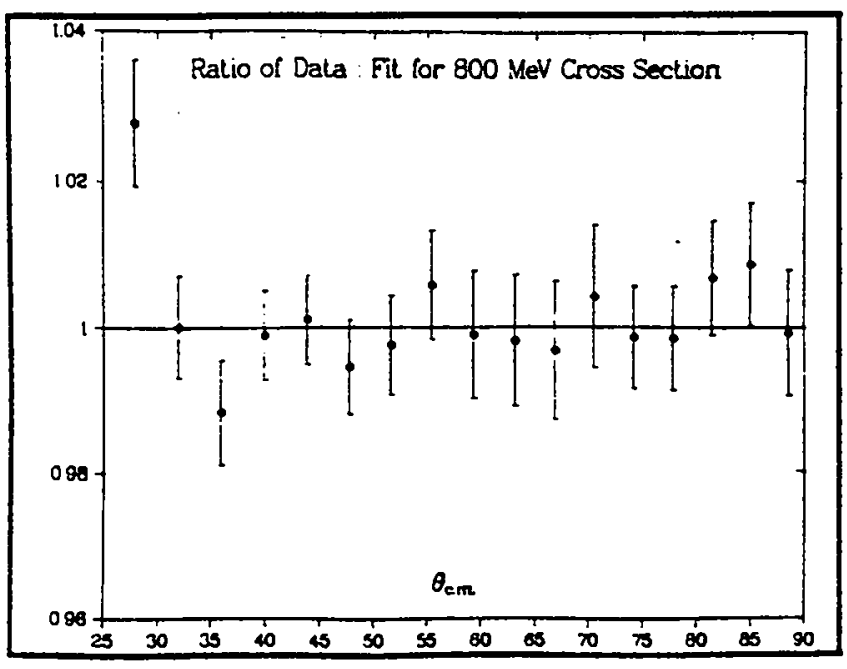

Fig. II-14. Ratio of the $800 \mathrm{MeV}$ pp elastic cross section to the phase-shift solution of Bugg. The uncertainty in the data is significantly smaller than $1 \%$.

these measurements $(0.5 \%)$ is at least ten times better than previous measurements above $600 \mathrm{MeV}$. Likewise, the agreement between the data and the phase-shift solution for this observable is now much better than $1 \%$.

Precise polarized-neutron calibrations awaited the high-intensity polarized source OPPIS, which began routine operation in 1990 . The $2 \mathrm{H}(\mathrm{p}, \mathrm{n}) 2 \mathrm{p}$ reaction will provide an important calibration standard for neutron polarimetry (worldwide) since the cross-section is large, background is small, and high resolution is not needed. At zero degrees, the spin-transfer is enhanced with respect to free $\mathrm{np}$ by the spin-zero diproton, and the process is easily calculated directly from the NN amplitudes. Preliminary results give excellent agreement with calculations based on the Bugg phase-shift analysis, but predictions based on other analyses such as Arndt's show $20 \%$ discrepancies. The disagreement is illustrated in Fig. II-11, where the two phase shift analyses are seen to diverge above $500 \mathrm{MeV}$. This disagreement is startling, since the two analyses are based on largely the same data, and may reflect the importance of how the inelasticities are handled. This example also underscores the importance of testing the free and effective NN amplitudes in kinematic regimes relevant to inelastic proton scattering.

This last phase of the free nucleon-nucleon scattering program at LAMPF is extremely important. Completion of this program will establish the foundation for a more detailed understanding of both the free and effective nucleon-nucleon interaction.

\section{Electroweak Probes}

\section{Introduction}

Electroweak theory has become sufficiently firm that electroweak interactions are emerging as specific probes of the strong interaction, especially at low energies. In addition, experimental techniques have developed to the extent that these probes are becoming precision tools. Because the electroweak couplings to quarks are understood, electroweak probes are particularly appropriate to attack the more complex problem, namely the coupling to real hadrons. For example, muon capture in polarized ${ }^{3} \mathrm{He}$ is being studied to measure the induced pseudoscalar form factor, which arises from the hadronic structure of the nucleus. Neutrino-proton elastic scattering is an ideal process with which to measure the contribution of strange quarks to the nucleon spin.

It has long been appreciated that the photon is an important probe in hadron physics. The reasons why the photon is a good hadronic probe are even more applicable to vector bosons. Until recently, limitations on achievable precision have limited the usefulness of electroweak probes. Recent and upcoming work at LAMPF demonstrates the power and breadth of using electroweak interactions to study hadronic physics.

This section describes a number of strong-interaction studies using electroweak probes. These studies would not be possible without the intense beams available at LAMPF.

\section{Measuring the Quark Contribution to the Proton Spin through $v p \rightarrow v p$}

\section{$v p \rightarrow v p$ Formalism}

The vp $\rightarrow$ vp cross section depends upon three form factors, $F_{1}, F_{2}$, and $G_{1}$. All three form factors are well known at $q^{2}=0$, from measurements with electromagnetic probes, except for the strange quark contributions; $F_{1}(0)=0.034, F_{2}(0)=1.017-F_{2}^{s} / 2$, and $G_{1}(0)=-g_{A} \sqrt{2}+G_{1}^{S} / 2$, where $g_{A}=1.26$, as known from neutron decay. The form factors are assumed to have a dipole form of $q^{2}$ dependence. At low $q^{2}$, the cross section is given by

$$
\frac{d \sigma}{d q^{2}}-\frac{G_{f}^{2}}{2 \pi}\left[\left(-0.63+\frac{G_{1}^{s}}{2}\right)^{2}\left(1+\frac{q^{2}}{4 E_{v}^{2}}\right)+(0.034)^{2}\left(1-\frac{q^{2}}{4 E_{v}^{2}}\right)\right],
$$

where the only unknown term is $G_{1}^{s}=\Delta s$, the strange quark contribution to the proton spin.

Thus, a measurement of $v p \rightarrow v p$ at low $q^{2}$ determines $\Delta s$. (We use the convention that $\Delta q$ is the $q$ 
quark contribution to the proton spin.) Polarized deepinelastic scattering experiments measure the integral

$$
\int g_{1}^{P}(x) d x=\frac{5}{18}(\Delta u+\Delta d)+\frac{1}{9} \Delta s+\frac{1}{6} g_{A} .
$$

and three years ago the EMC experiment measured the value of this integral to be $0.126 \pm 0.010 \pm 0.015$, in disagreement with the Ellis-Jaffe sum rule prediction of $0.189 \pm 0.005$. SU(3) symmetry has been invoked to interpret this result to imply that $\Delta s$ is nonzero and that $\Sigma \Delta q=\Delta \mathbf{u}+\Delta d+\Delta s \sim 0$. However, Manohar, Kaplan, and Lipkin have recently argued that SU(3) symmetry is suspect and that it may be invalid for SU(3) symmetry to relate matrix elements of charged and neutral axial currents. Therefore, $v p \rightarrow$ vp and polarized deep-inelastic scattering experiments are very complementary. vp $\rightarrow$ vp measures $\Delta s$, polarized deep-inelastic scattering measures $(5 / 18)(\Delta u+\Delta d)+(1 / 9) \Delta s+(1 / 6) g A$. Combining the two determines $\Sigma \Delta \mathrm{q}$.

\section{The LSND Experiment}

The Liquid Scintillator Neutrino Detector (LSND) experiment consists of a cylindrical tank of dilute mineraloil-based liquid scintillator approximately $6 \mathrm{~m}$ in diameter by $9 \mathrm{~m}$ long with an active mass of 200 tons. There will

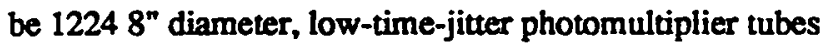
mounted uniformly over the inside surface $(25 \%$ coverage). The tank will reside inside an existing veto shield, located $28 \mathrm{~m}$ downstream of the A6 proton beam stop and at an angle of approximately $13^{\circ}$ to the beam direction. An upgraded A6 beam stop is now being considered, which would produce a factor of five more decay-in-flight neutrinos and, therefore, five times more $v p \rightarrow v p$ events.

Figure II-15 shows the vp $\rightarrow$ vp elastic scattering cross section as a function of neutrino energy. At LAMPF the average decay-in-flight neutrino energy is about $150 \mathrm{MeV}$, where the cross section is $3 \times 10^{-40}$ $\mathrm{cm}^{2}$ and the maximum $\mathrm{q}^{2}$ is $0.07 \mathrm{GeV}^{2} / \mathrm{c}^{2}$. The neutrino energies available at LAMPF are optimal; the energy is sufficiently high that the cross section is almost at saturation but sufficiently low that the low $q^{2}$ approximation, $q^{2} \ll m_{p}^{2}$, is valid. Folding in the neutrino decay-in-flight energy spectrum, the overall recoil proton kinetic energy distribution is shown ut Fig. II-16. Two thousand vp $\rightarrow$ vp events per year are expected with the present beam stop and 10,000 events per year with the upgraded beam stop.

The main extrinsic background is due to cosmic-ray induced neutrons with an estimated rate of 2000 per year. This background is large but can be easily determined by

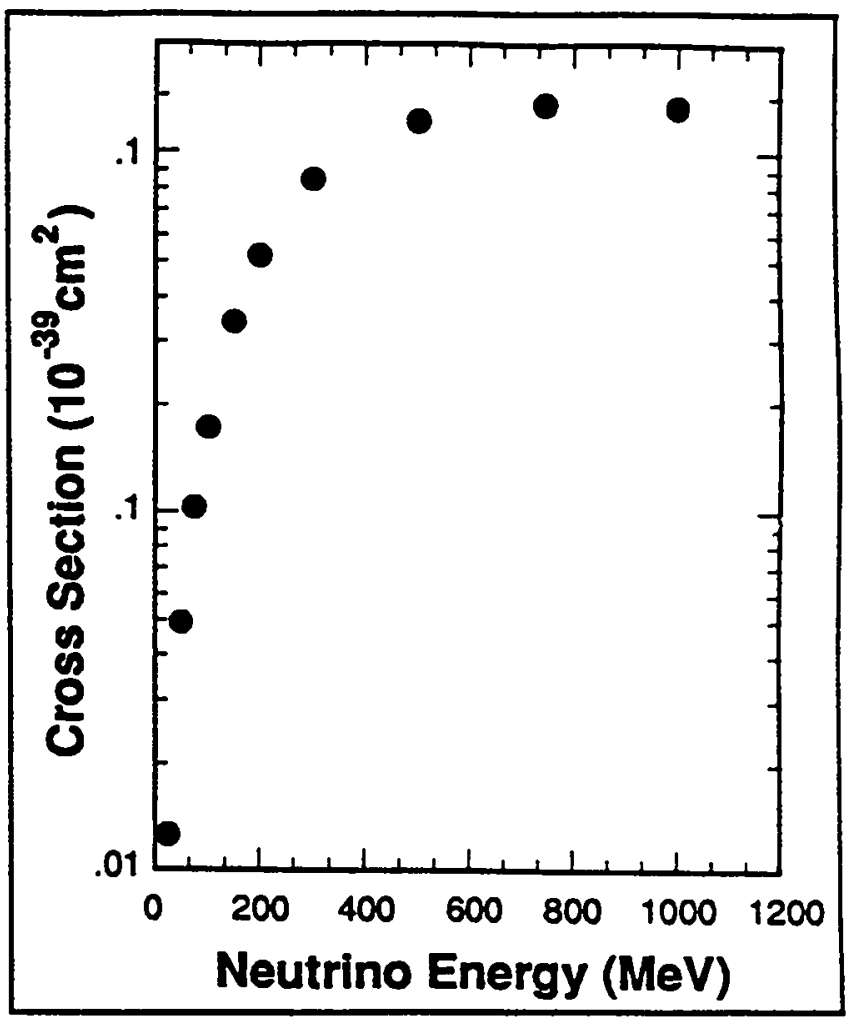

Fig. II-15. The $v_{p} \rightarrow v_{p}$ elastic scattering cross section as a function of neutrino energy.

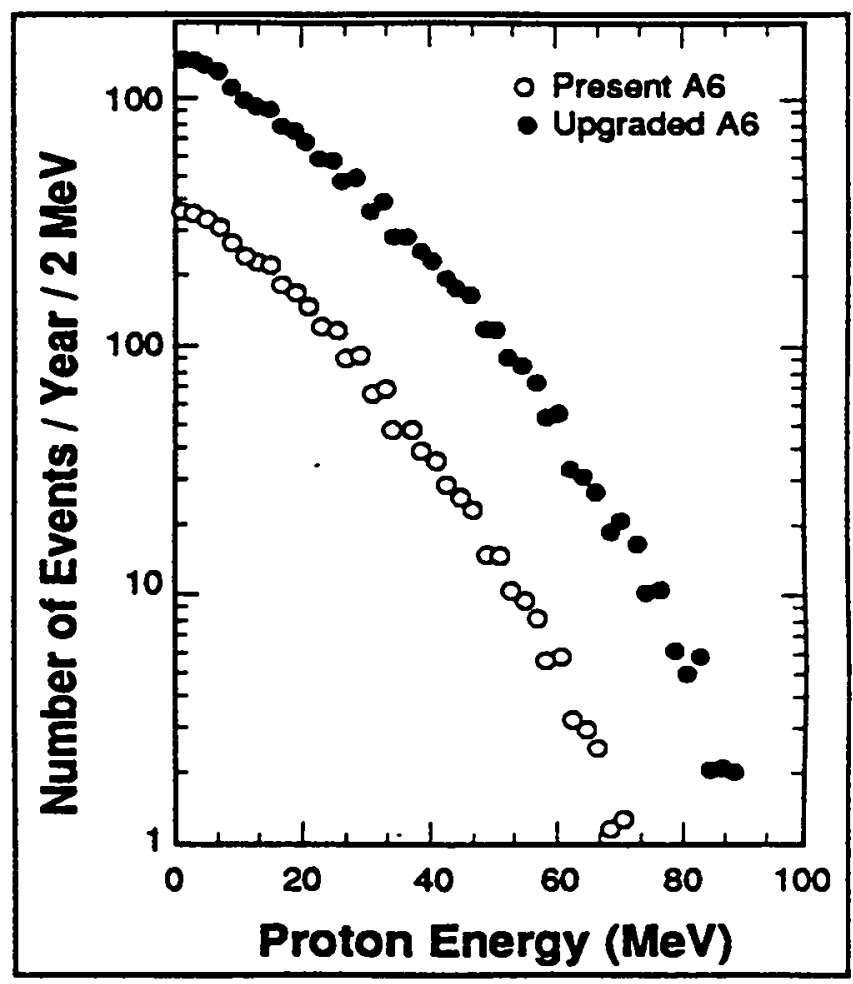

Fig. II-16. The overall recoil proton kinetic energy distribution from $v p \rightarrow v p$ after folding in the LAMPF neutrino energy spectrum. 
subtracting data taken with the beam off. The main intrinsic background is $\mathrm{VC} \rightarrow \mathrm{pX}$, neutrino scattering from a bound proton, which is estimated to occur at a rate comparable to vp $\rightarrow v p$. The best method for dealing with this bound proton background is to run half of the time with $\mathrm{CH}_{2}$ scintillator (mineral oil based), the other half with $\mathrm{CH}_{1.3}$ scintillator (pseudo-cumene based), and perform a $\mathrm{CH}_{2}-\mathrm{CH}_{1.3}$ subtraction to extract the neutrino cross section from free protons. With the present beam stop and with a $10-\mathrm{MeV}$ recoil proton energy threshold, the $\mathrm{CH}_{2}-\mathrm{CH}_{1.3}$ subtraction will yield $700 \pm 85 \pm 63$ events, where the first error is the statistical error due to the scintillator subtraction and the second error is the statistical error due to the beam-on minus beam-off subtraction. With a normalization error of $\pm 6 \%$ (statistical) and $\pm 7.5 \%$ (systematic), the total error on the cross section is $\pm 18 \%$, which corresponds to a \pm 0.11 error on $\Delta s$. For an upgraded beam stop with the same 10$\mathrm{MeV}$ recoil proton energy threshold, the total error on the cross section is estimated to be $\pm 8.8 \%$, corresponding to a \pm 0.05 error on $\Delta s$. Such a measurement, therefore, will determine whether strange quarks contribute substantially to the spin of the proton and, together with polarized deepinelastic scattering experiments, resolve the question of how quarks contribute to the proton spin.

\section{Study of the Induced Pseudoscalar Form Factor using Polarized ${ }^{3} \mathrm{He}$}

The spin dependence of nuclear reactions is emerging as one of the vital tools for nuclear physics studies involving the strong, electromagnetic, and weak interactions. Recent developments in polarized beams, polarized targets, and polarimeters have all contributed to this activity. At LAMPF, a technology is being developed to allow the spin dependence of negative muon capture to be measured. The most important example is negative muon capture on ${ }^{3} \mathrm{He}$ resulting in a triton and a neutrino. The spin dependence provides a measure of the poorly determined induced pseudoscalar form factor $\mathrm{g}_{\mathrm{p}}$. We note that muon capture (spin dependent or radiative) is the only way to probe this physics.

The easiest way to produce polarized muonic atoms is to stop polarized muons. Unfortunately, negative muons are strongly depolarized as they cascade down to the ground state from which nuclear capture occurs. For ${ }^{3} \mathrm{He}$, polarizations of only a few percent result even with fully polarized incident muons. This small polarization makes such nuclear capture experiments very insensitive to spin. An alternative approach, using lasers to polarize the muonic atoms, is being investigated. There is also interest in atomic physics processes that may influence the spin state of muonic atoms. This knowledge is of course essential for producing highly polarized samples of muonic atoms useful for nuclear physics.

The first approach tried was to stop unpolarized muons in a laser polarized ${ }^{3} \mathrm{He}$ target. It was found that muonic ${ }^{3} \mathrm{He}$ ions with a polarization of $7 \%$ result from a $100 \%$ polarized target. This number is about a factor of two lower than predicted by the theory of cascade depolarization, and indicates that collisions during the cascade depolarize the system. This is a very interesting result, but there was hope for an unexpectedly large polarization. Recently, the value of a radically different approach, namely polarizing the muonic helium in its ground state after it has formed but before the end of its 2.2- $\mu$ s lifetime, has been demonstrated. In this method, negative muons are stopped in a glass cell containing the ${ }^{3} \mathrm{He}$ as well as about $10^{15}$ atoms/cc of $\mathrm{Rb}$ vapor which has been polarized by a laser. Partially polarized neutral muonic ${ }^{3} \mathrm{He}$ is produced, picking up a polarized electron from the $\mathrm{Rb}$. This paramagnetic atom is further polarized by spin-exchange collisions with the $\mathrm{Rb}$. The time dependence of the muon polarization enables the cross sections for the reactions involved to be measured, which yields important information for atomic physics.

An exciting feature of this new method is the high polarizations that result. After a few muon lifetimes, the triplet ions become essentially fully polarized, and average polarizations of about $25 \%$ result. This represents an order of magnitude improvement over the best polarizations previously obtained. It appears that this method will be ideal for an experiment to measure the spin-dependence of nuclear muon capture in ${ }^{3} \mathrm{He}$. The high polarizations available result in both vector and tensor polarized muonic helium, each of which provides independent information in the capture process. Detecting the recoil tritons and measuring their direction represents an experimental challenge since the tritons do not have enough energy to leave the target. Thus, the target must also be instrumented as a detector. The approach used is to make the target also serve as a drift chamber. An apparatus to exploit these possibilities is being developed to measure asymmetries in the near future.

LAMPF is an ideal facility for this program. The SMC provides a very intense source of muons, and has instrumentation that aliows a clean beam of muons to be produced with the proper phase space for stopping in small, thin targets. Indeed, meeting the requirement of stopping muons in a $2.5-\mathrm{cm}$-diameter glass cell with 8 atm of helium was one of the great technical challenges of the experiment. The support for these experiments (laser utilities, beam line modifications, etc.) has been excellent. This program has been well suited to the training of 
students. The experiments are short enough in time and small enough so that each student can participate in all pliases, from writing the proposal to writing the final paper, and can be familiar with all experimental aspects. Two theses are being written based on our past results, two more students are planning to do theses, and a total of more than ten students have gained significant experience working on this program. The students' enthusiasm has been translated into an effective learning experience and contributions to physics.

\section{Neutrino Carbon Neutral Current Interactions}

The interaction ${ }^{12} \mathrm{C}\left(v, v^{\prime}\right)^{12} \mathrm{C}^{*}\left(1^{+}, 1: 15.11 \mathrm{MeV}\right)$ is one of the few neutral current nucleus interactions that is experimentally accessible. An accurate measurement of the cross section at neutrino energies below $53 \mathrm{MeV}$ and above $80 \mathrm{MeV}$ will provide information on neutral current interactions in nuclei and on nuclear form factors. A precision measurement of this process is especially important because is allows a calibration of the calculations used in current models of neutrino-nucleus interactions during supernova bursts.

A recent calculation of the total cross section is given in Fig. II-17. Below $53 \mathrm{MeV}$ the cross section is dominated by contributions from $F_{A}$, the nuclear axial vector form factor, while for neutrino energies above 100 $\mathrm{MeV}$ contributions from $\mathrm{F}_{\mathrm{M}}$ and $\mathrm{F}_{\mathrm{E}}$ enter at about the $6 \%$ level. $F_{M}$ and $F_{E}$ are the weak magnetism and electric form factors, respectively.

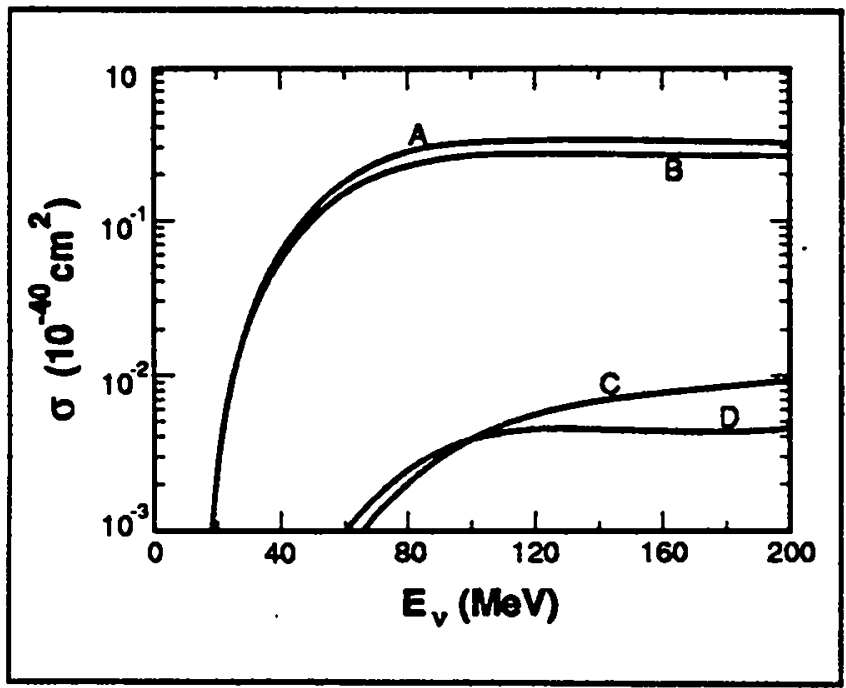

Fig. II-17. Plot of the total cross section for $v_{e}\left(v_{\mu}\right)$ and $\bar{v}_{\mu}\left(\bar{v}_{e}\right)$ neutral-current reaction on ${ }^{12} C$ as a function of incoming neutrino energy. Curves $(B),(C)$, and $(D)$ refer to the contribution from $F_{A}, F_{E}$, and $F_{M}$, respectively, for these reactions.
Recently the KARMEN experiment at the Rutherford Appleton Laboratory reported on the first observation of ${ }^{12} \mathrm{C}\left(\nu, v^{\prime}\right)^{12} \mathrm{C}^{*}$. The experiment uses $\bar{v}_{\mu}$ and $v_{e}$ from $\mu^{+}$ decay at rest and detects the neutrino induced events is a 56-ton segmented liquid scintillator detector. Based on the observation of $21.5 \pm 10.1$ events they measured a total cross section of:

$\left(\bar{v}_{\mu}+v_{e}\right)=\{10.8 \pm 5.1($ stat $) \pm 1.1($ syst $)\} \times 10^{-42} \mathrm{~cm}^{2}$

Ultimately the KARMEN experiment expects to measure this cross section with an accuracy of $\pm 10 \%$.

The LSND experiment will identify the reaction ${ }^{12} \mathrm{C}\left(v, v^{\prime}\right)^{12} \mathrm{C}^{*}$ by detecting the $15.11-\mathrm{MeV}$ decay photons with an energy resolution of better than $10 \%$. LSND will detect about 1300 events per year from $\pi^{+}$and $\mu^{+}$decay at rest $\left(E_{n}<53 \mathrm{MeV}\right)$ and about 220 events per year from $\pi^{+}$decay in flight $\left(80<\mathrm{E}_{V}<200 \mathrm{MeV}\right)$. With the proposed upgrade to the $\mathrm{A} 6$ beam stop, the decay in flight rate will increase to $800 /$ year. The events from $\pi^{+}$ decay in flight can be partially separated from the decay at rest events using the $200-\mathrm{MHz}$ structure of the LAMPF beam. The main backgrounds for the decay at rest measurement are cosmic ray induced events (100/year) and VC charged current events (100/year). The cosmic-ray background will be determined from beam-off data and the vC charged current background will be determined by fitting to the data above $20 \mathrm{MeV}$. The cross section integrated over the decay-at-rest neutrino spectrum will be measured with a statistical accuracy of $5 \%$ in one year. The systematic errors are expected to be less than 5\%. The dominant error for the decay-in-flight measurement will be events produced by decay-at-rest neutrinos (260/year). Without the proposed A6 upgrade, the cross section from decay-in-flight neutrinos can be measured to $17 \%$ in one year. With the upgrade, a $7 \%$ measurement is possible in one year.

The intense and well-characterized neutrino flux at LAMPF will allow the LSND experiment to detect 1500 2000 neutrino carbon neutral current events per year. With this number of events, LSND will be able to measure the cross section to better than 5\% within two years. With the proposed upgrade to the $A 6$ beam stop the energy dependence of the cross section can be tested with 5\% measurements for neutrino energies below $53 \mathrm{MeV}$ and above $80 \mathrm{MeV}$.

\section{Experimental Study of Neutrino Absorption on Carbon}

Low-energy neutrino-nuclear reactions present challenges and opportunities for investigations at the 
intersections of particle, nuclear, and astrophysics. Prior to the E225 experiment at LAMPF, published measurements of absolute cross sections to unbound nuclear final states have been limited to deuteron break-up reactions: the $d\left(v_{e}, e^{-}\right)$pp reaction, by LAMPF experiment E31, with muon-decay neutrinos; and the ratio of $d\left(\vec{v}_{e}, e^{+}\right) n n$ to $\mathrm{d}\left(\bar{v}_{\mathrm{e}}, \overline{\mathrm{v}}_{\mathrm{e}}\right) \mathrm{pn}$ reactions with reactor antineutrinos.

The E225 collaboration (UC Irvine, Los Alamos, Argonne, Maryland and Tel Aviv) measured the exclusive reaction ${ }^{12} \mathrm{C}\left(v_{\mathrm{e}}, \mathrm{e}^{-}\right)^{12} \mathrm{~N}(\mathrm{~g} . \mathrm{s}$.$) and the inclusive reaction$ ${ }^{12} \mathrm{C}\left(v_{e}, e^{-}\right) \mathrm{X}$ at energies $\left(E_{V} \approx 20-50 \mathrm{MeV}\right)$ relevant to cosmic neutrino observatories and to particle and nuclear physics investigations. In addition to its intrinsic scientific interest, this measurement represents a proof-ofprinciple demonstration of the feasibility of a new class of experiments in weak nuclear interactions. (The reaction ${ }^{12} \mathrm{C}\left(v_{\mathrm{e}}, \mathrm{e}^{-}\right)^{12} \mathrm{~N}(\mathrm{~g} . \mathrm{s}$.$) and the related neutral current$ excitation of carbon, ${ }^{12} \mathrm{C}\left(v, v^{\prime}\right)^{12} \mathrm{C}(15.1)$, have recently been measured by an experiment operating at the Rutherford ISIS beam stop; the reaction ${ }^{12} C\left(v_{\mu}, \mu^{-}\right) X$, measured at the LAMPF Line E facility, will soon be published.)

The exclusive reaction between specific nuclear states, ${ }^{12} \mathrm{C}\left(v_{e}, e^{-}\right)^{12} \mathrm{~N}(\mathrm{~g}$. s. $)$, was measured by observation of the prompt electron in delayed coincidence with the subsequent $12 \mathrm{~N}$ (g.s.) decay positron. Investigation of inclusive neutrino-carbon reactions was performed using detailed measurements of the angular and energy distributions of the prompt ("recoil") electrons. The detector was a fine-grained tracking calorimeter comprised of plastic scintillation counters and flash chamber tracking detectors.

The experiment was performed at the LAMPF beam stop, where the $800-\mathrm{MeV}$ proton beam provides an intense source of electron-neutrinos from stopped-muon decay. The absolute flux normalization was calculated by a detailed Monte Carlo simulation of pion production, absorption, and decay in the proton beam stop; the simulation was calibrated by an ancillary measurement that achieved systematic normalization uncertainties smaller than $6 \%$ for stopped-pion production by protons in an instrumented simplified beam stop. Measurement of the proton current with $2 \%$ absolute precision, the scaling of the Monte Carlo to the calibration data, and the extrapolation to the actual complex beam stry configuration and geometry increased the total flux uncertainty to $7.3 \%$.

The ${ }^{12} \mathrm{C}\left(v_{\mathrm{e}}, \mathrm{e}^{-}\right)^{12} \mathrm{~N}(\mathrm{~g} . \mathrm{s})$ signal was identified by ${ }^{12} \mathrm{~N}$ (g.s.) decay positrons in delayed-coincidence with prompt (trigger) electrons satisfying the energetic limits for ${ }^{12} \mathrm{C}\left(v_{\mathrm{e}}, \mathrm{e}^{-}\right)^{12} \mathrm{~N}$ reactions. Transitions to excited states of nitrogen were not included in the delayed positron coincidence because all of these states are particleunstable.

The observation of $181.5 \pm 17.1^{12} \mathrm{~N}$ (g.s.) decay events gives a measured neutrino-flux weighted exclusive cross section of

$$
\begin{aligned}
& \left\langle\sigma\left({ }^{12} \mathrm{C}\left(v_{e}, e^{-}\right)^{12} \mathrm{~N}(\mathrm{~g} . \mathrm{s} .)\right)\right\rangle= \\
& \quad[1.05 \pm 0.10 \text { (stat) } \pm 0.10(\text { syst })] \times 10^{-41} \mathrm{~cm}^{2} .
\end{aligned}
$$

The statistical error includes the systematic uncertainties introduced by the fitting procedure, and the systematic errors include the uncertainties in the overall detection efficiency (6.4\%), the neutrino flux (7.3\%) and the target composition (2\%) added in quadrature. If one assumes that the neutrino-absorption cross section above threshold $Q$ has a $\left(E_{v}-Q\right)^{2}$ dependence on the neutrino energy $E_{v}$, then the flux-weighted cross section obtained with muondecay neutrino spectrum is equivalent to the cross section for monoenergetic neutrinos with $E_{V}=35 \mathrm{MeV}$.

Inclusive $v_{e}{ }^{12} \mathrm{C}$ reactions to ${ }^{12} \mathrm{~N}$ excited states do not have a delayed-time signature. The inclusive events were identified solely by the kinematic distributions of the prompt electrons and their differences relative to the background processes. The beam-associated events were presumed to be the sum of contributions from the neutrino-carbon inverse-beta-decay signal of interest here, and several backgrounds, namely neutrino-electron elastic scattering, neutrino absorption by other isotopes, and neutron-induced gamma-rays. The expected numbers of events, $\mathbf{N}_{\mathrm{ev}}$, for each of the neutrino-induced backgrounds and for the ${ }^{12} \mathrm{C}\left(v_{e}, e^{-}\right) \mathrm{X}$ signal have been estimated from the calculated beam-weighted cross section $\langle\sigma\rangle$, integrated number of target nuclei or electrons $T$, estimated acceptance $\langle\varepsilon\rangle$, and neutrino fluence $\phi$, according to $\mathrm{N}_{\mathrm{ev}}=$ $\langle\varepsilon\rangle \phi T\langle\sigma\rangle$. These quantities are listed in Table II- 1 .

The ${ }^{12} \mathrm{C}\left(v_{e}, e^{-}\right) \mathrm{X}$ signal was extracted from the data, and the backgrounds were determined empirically by a multiparameter maximum likelihood fit to the visible energy and observed recoil angle $\left(E_{v i s}-v s-\cos \theta_{e v}\right)$ distributions. In Fig. II-18, the normalized ${ }^{12} \mathrm{C}\left(v_{e}, e^{-}\right) \mathrm{X}$ distribution is plotted with the data remaining after all cosmic-ray and beam-associated backgrounds have been subtracted according to the results of the fit. The extracted ${ }^{12} \mathrm{C}\left(v_{\mathrm{e}}, \mathrm{e}^{-}\right) \mathrm{X}$ signal is not sensitive to the detailed assumptions about the background component distributions.

Evaluation of the ${ }^{12} \mathrm{C}\left(v_{\mathrm{e}}, \mathrm{e}^{-}\right) \mathrm{X}$ cross section depends upon the integrated detection efficiency for the recoil electrons, $\varepsilon=7.6 \pm 0.4 \%$, as weighted according to calculated transition strengths. The inclusive $v_{e}$ absorption cross section for ${ }^{12} \mathrm{C}$ is 
Table II-1. Sources of events in the final data sample. The fourth row represents neutron-induced gamma-ray background events. The "Expected Number of Events" is evaluated for $\phi=9.16 \times 10^{14} \mathrm{~cm}^{-2}$ electron-neutrinos. The ${ }^{12} \mathrm{C}\left(v_{e}, e^{-}\right) \mathrm{X}$ total cross section measurement is based on the number of events determined by the "Best Fit" shown in the right-most column.

\begin{tabular}{|lcccc||c|}
\hline \multicolumn{1}{|c}{ Reaction } & $\begin{array}{c}\text { Targets } \\
\mathrm{T}\left(10^{29}\right)\end{array}$ & $\begin{array}{c}\langle\sigma\rangle \\
10^{-42} \mathrm{~cm}^{2}\end{array}$ & $\begin{array}{c}\langle\varepsilon\rangle \\
\%\end{array}$ & $\begin{array}{c}\text { Expected } \\
\text { Number of Events }\end{array}$ & Best Fit \\
\hline$v_{e}{ }^{12} \mathrm{C}$ & 6.36 & 13.2 & 7.6 & 583 & $626 \pm 71$ \\
$\left(v_{e}+v_{\mu}+\bar{v}_{\mu}\right) \mathrm{e}^{-}$ & 49.5 & 0.4 & 15.7 & 284 & $295 \pm 35$ \\
& & & & & \\
$v_{e}\left({ }^{13} \mathrm{C}+{ }^{27} \mathrm{Al}\right)$ & 0.17 & 116.1 & 15.6 & 282 & $136 \pm 102$ \\
$\mathrm{np} \rightarrow \gamma$ & & & & & $435 \pm 90$ \\
\hline
\end{tabular}

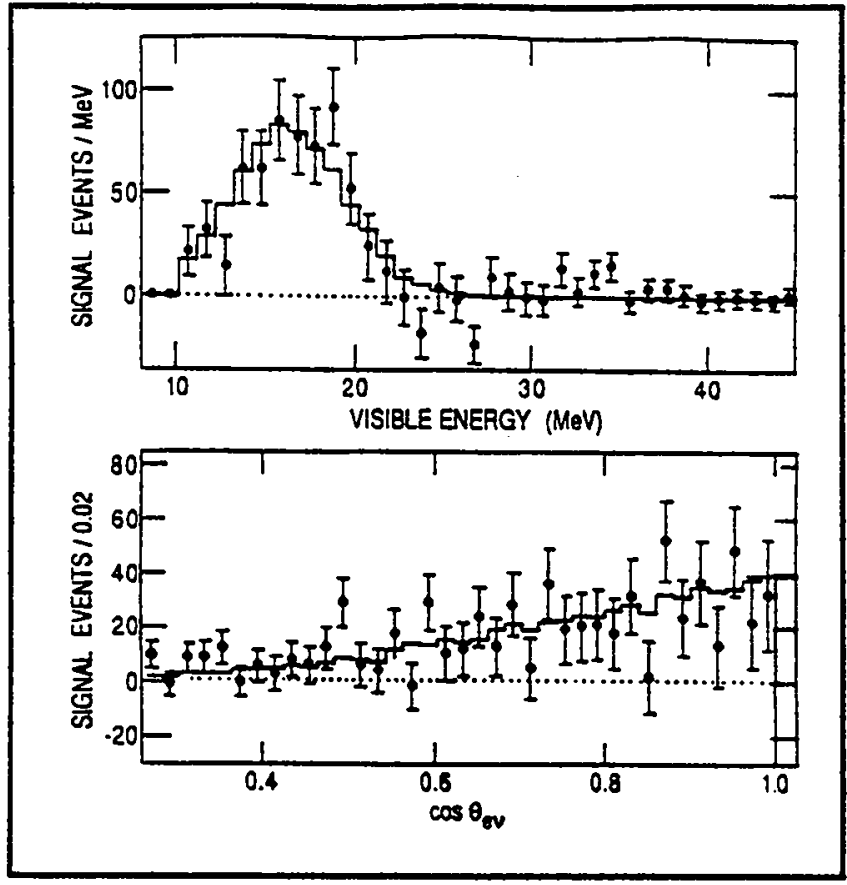

Fig. II-18. Comparison of Monte Carlo expectation for ${ }^{12} C\left(v_{e}, e^{-}\right) X$ signal (histogram), normalized to results of fit, compared to data (points with errors) afier all cosmicray and beam-associased backgrounds have been subtracted. (top) is the visible energy. (bottom) is the cosine of angle between incident neutrino and measured electron track.

$$
\begin{aligned}
& \left\langle\sigma\left({ }^{12} \mathrm{C}\left(v_{e}, e^{-}\right) X\right)\right\rangle= \\
& \quad(1.41 \pm 0.16(\text { stat }) \pm 0.19(\text { syst })) \times 10^{-41} \mathrm{~cm}^{2}
\end{aligned}
$$

where the (syst)-error is the quadrature sum of the uncertainties in the Monte Carlo simulation (5\%), neutrino flux (7.3\%), target density (2\%), and an estimate of the uncertainty in overall detection efficiency $\varepsilon$ introduced by ambiguities in the relative weighting of the nitrogen ground-state and various excited-state contributions to the total event rate $(7 \%)$. With the relative normalization of each contribution unconstrained, the fit indicates a $(65 \% / 35 \%)$ split between events to the nitrogen ground state and to nitrogen excited states. Consistent values of the cross section were obtained for all hypothesis about the final state distribution.

Calculations of the cross sections generally involve a small set of multipole operators producing transitions between, for example, appropriately weighted sums of single-particle states. The $\mathrm{q}^{2}$ dependence of the nuclear form factors can be constrained by using existing electron scattering, muon capture, and beta-decay measurements, which are then related to the neutrino absorptions through CVC and weak-isospin transformations. This measurement is in good agreement with the theoretical calculations, for example, those of T. W. Donnelly, which confirms that the complementary nuclear transition data can be used to predict the neutrino-induced reaction rate.

\section{Parity and Time-Reversal Violation in the Compound Nucleus}

The study of parity violation in the compound nucleus has been going on at the Los Alamos Neutron Scattering Center (LANSCE) for approximately 5 years. Parity-violating longitudinal asymmetries are measured for $\mathrm{p}_{1 / 2}$ compound-nuclear resonances using longitudinally polarized neutrons. These asymmetries are defined as the fractional difference of the cross sections for exciting a resonance with neutrons having positive and negative helicities. This study was motivated by the early work at Dubna in which very large, few percent, parity-violating longitudinal asymmetries were observed for compound nucleus resonances. The small level spacings and large ratios of $s$-wave to $p$-wave decay amplitudes amplify the size of parity violating effects by factors as large as $10^{6}$. 
The original motivation for this work was the desire to take advantage of the large enhancement of symmetry violation to design a sensitive search for a violation of time-reversal invariance. In the process, we have discovered new phenomena in parity violation and made theoretical advances that allow us to relate the observed effects to the underlying weak nucleon-nucleon interaction.

This work uses the polarized neutron beam at LANSCE, a spallation source driven by the $800-\mathrm{MeV}$ proton beam from LAMPF. This source is by far the world's most intense source of pulsed, epithermal neutrons. The nearest competitor is the KENS spallation source at KEK; for experiments such as these, the flux at LANSCE is a factor of 30 larger than at KEK. The comparison with the ORELA source at Oak Ridge National Laboratory is even more dramatic: over the energy region from 1 to $1000 \mathrm{eV}$, the flux at LANSCE is 2 to 3 orders of magnitude larger than at ORELA. The superiority of the Los Alamos facility is demonstrated by the fact that we have measured more cases of parity violation in a single sample ( $\left.{ }^{232} \mathrm{Th}\right)$ than had previously been measured in all neutron resonances, anywhere in the world!

Because of the large neutron flux, parity violation in a large number of neutron resonances in a given nucleus can be measured. The large data set on ${ }^{232} \mathrm{Th}$ shown in Fig. II-19 allows systematic studies. Although the

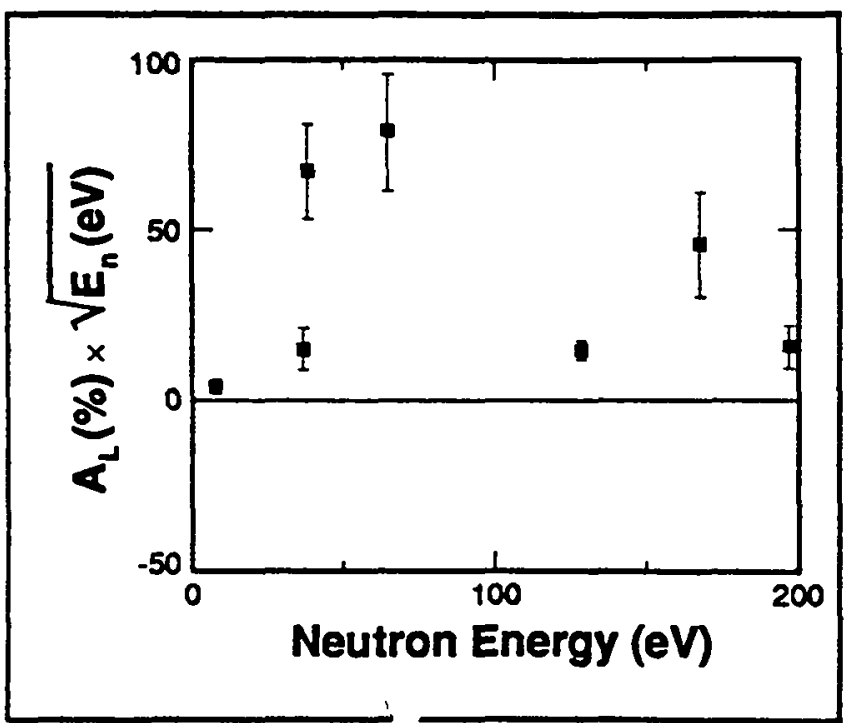

Fig. II-19. Asymmetry data for $p_{1 / 2}$ resonances in ${ }^{232} T h$. The horizontal aris is the incident neurron energy in $\mathrm{eV}$ and the vertical axis is the measured asymmetry times the square root of the neutron energy. The measured asymmetries show both a nonzero average value and fluctuations about this value. statistital description of the compound nucleus predicts that the signs of the parity-violating asymmetries will be random, the data on Th show that all seven resonances that have asymmetries with greater than 2 sigma significance have positive asymmetries. When the data for all nuclei are examined, 13 of 15 significant effects have a positive sign. This systematic effect forced development of an model of parity violation in the compound nucleus involving two kinds of parity mixing. Opposite-parity compound-nuclear levels a few eV away from the level under study are admixed according to matrix elements of the weak interaction having a statistical character. These admixtures produce the fluctuations. Opposite-parity states several MeV away are admixed according to matrix elements having a single-particle character to produce an average, positive, asymmetry.

Using statistical techniques, the mean square statistical parity-violating matrix element $M 2$ from the fluctuations in the measured asymmetries can be extracted. In parallel with the experimental work, Johnson et al. developed a model relating $\mathbf{M}$ to the meson couplings of the weak nucleon-nucleon interaction. The weak rhonucleon coupling was determined to an accuracy of $30 \%$. The second, unexpected, feature of the asymmetry data is the nonzero average asymmetry. The size of the singleparticle matrix elements necessary to explain the average asymmetry is approximately $30 \mathrm{eV}$, thirty times larger than naively expected. The reaction theory needed to extract the single-particle matrix element is being worked out now, but if the large value of the single-particle matrix holds up, our thinking about weak interactions in nuclei will be fundamentally altered.

The sensitivity of the apparatus is being improved by a factor of twenty. These improvements will allow the more extensive and accurate measurements needed to determine matrix elements and couplings more accurately and to test reaction models.

In addition to this fruitful study of parity violation, active planning for the first measurements searching for violation of time-reversal invariance is under way. A new neutron spin filter that will increase the figure of merit by an order of magnitude is about to be brought on line. The measurements will have a sensitivity at least two orders of magnitude below the best limits on violation of timereversal invariance in the strong interaction. The LANSCE facility at LAMPF is the only place in the world where these experiments are feasible.

\section{Parity Nonconservation in Proton Scattering}

The first experiments to search for parity nonconservation in nucleon-nucleon scattering used 
double-scattering or triple-scattering geometries. This technique was limited to a precision of $-10^{-3}$. A new generation of experiments began at LAMPF with a proposal to measure the helicity dependence of the transmission of $1.5-\mathrm{GeV} / \mathrm{c}$ longitudinally-polarized protons through an unpolarized target using integral counting techniques. An interference between the strong amplitude and the parity-nonconserving weak amplitude is expected to produce a longitudinal asymmetry $A_{L}=\left(\sigma_{+}-\right.$ $\left.\sigma_{-}\right) /\left(\sigma_{+}+\sigma_{-}\right)$at the level of $10^{-7}$, where $\sigma_{+}\left(\sigma_{-}\right)$is the total cross section for positive (negative) helicity protons.

Since then, experiments have been performed at four energies. The experiment using a $15-\mathrm{MeV}$ polarized beam at the Los Alamos Tandem Van de Graaff was begun in 1972. When a $6-\mathrm{GeV} / \mathrm{c}$ polarized beam became available at the Argonne ZGS, an experiment was started there and the $1.5-\mathrm{GeV} / \mathrm{c}$ experiment at LAMPF was begun in 1978 . These experiments, together with experiments at $45 \mathrm{MeV}$, sample the energy dependence of $A_{L}$ (see Fig. II-20).

Measurements of $A_{L}$ at 15 and $45 \mathrm{MeV}$ on hydrogen yield results in reasonable agreement with theoretical predictions based on a meson-exchange model and a hybrid quark model. On the other hand, the experiment with 6$\mathrm{GeV} / \mathrm{c}$ protons on a $\mathrm{H}_{2} \mathrm{O}$ target has reported a value $\mathrm{AL}_{\mathrm{L}}=$ $(26.5 \pm 6.0) \times 10^{-7}$ that is much larger than expected from calculations made prior to the experiment. Later, meson-exchange calculations have confirmed the prediction of $A_{L}-1.0 \times 10^{-7}$. Other theoretical approaches include the multi-peripheral model and heavy boson exchange, both of which also predict $A_{L}$ to be $\sim 10^{-7}$. The contribution from Coulomb effects is expected to give only a $15 \%$ enhancement of the asymmetry. Another calculation used Regge theory to

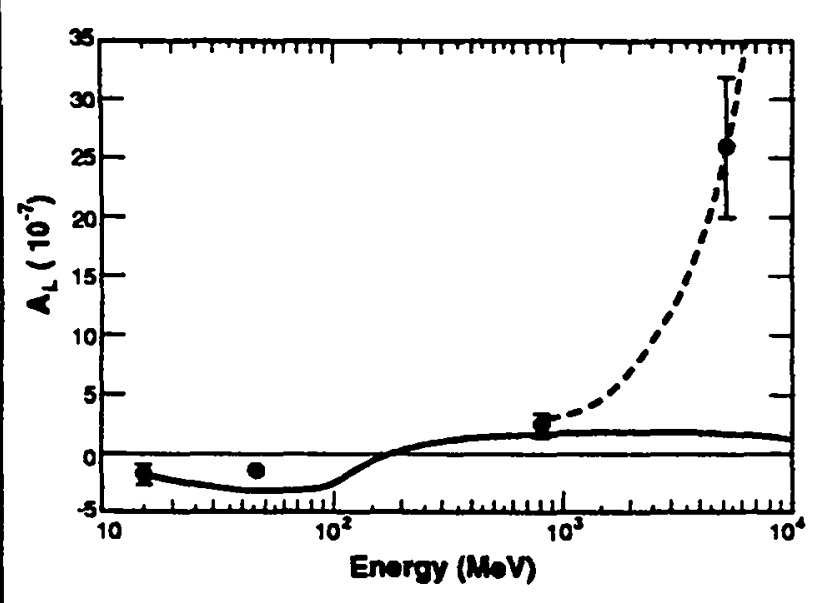

Fig. II-20. Measured values of AL versus energy. The solid curve is a generic meson-exchange calculation and the dashed curve is the quark level calculation. calculate the contribution to the asymmetry from parity nonconservation in the nucleon wave function. The result is $A_{L}=+2.1 \times 10^{-6}$ with an estimated error of $30 \%$, but this calculation has been criticized because its extension to low energies yields predictions for several paritynonconservation results that are much larger than experimental values.

A calculation has been reported that considers the effects of parity nonconservation at the quark level. This calculation included contributions from both the scattering and from the wave functions. The interaction takes place in the nucleon between one quark and a vector diquark. The results are dominated by the wave function contribution, with $A_{L}=+(7$ to 27$) \times 10^{-7}$. Although this model is expected to be valid only at high energy and the uncertainty is large, the result is very encouraging.

The experiments at $1.5 \mathrm{GeV} / \mathrm{c}(800 \mathrm{MeV})$ are at an energy intermediate to the previous measurements. Results have been published for polarized protons on an $\mathrm{H}_{2} \mathrm{O}$ target and for an $\mathrm{LH}_{2}$ target. These results can be compared with a surprisingly large range of values among published predictions for the asymmetry. The variation is mainly due to the use of different parameterizations of the strong nucleon-nucleon interaction. The predicted values for $A_{L}$ from meson-exchange models range from $(-8 \times$ $\left.10^{-7}\right)$ to $\left(+3 \times 10^{-7}\right)$ with intermediate values of $(-0.2 \times$ $\left.10^{-7}\right)$ and $\left(+2 \times 10^{-7}\right)$. A hybrid-quark model predicts a value $<1 \times 10^{-8}$, and the wave-function renormalization model predicts a large positive value $\left(+18 \times 10^{-7}\right)$. If the high-energy quark-quark model is extrapolated down to 1.5 $\mathrm{GeV} / c$, the expectation is $+2 \times 10^{-7}$.

The experiments performed at LAMPF utilized 1.5$\mathrm{GeV} / \mathrm{c}$ longitudinally polarized protons. The proton helicity was reversed with a $30-\mathrm{Hz}$, periodicity. The layout of the version of the experiment with a $1-\mathrm{m} \mathrm{LH}_{2}$ target is shown in Fig. II-21. The transmission of protons through various targets was measured by two integrating ion chambers (I1 and I2), located upstream and downstream of the target. For the two helicity states of the beam, the fractional change in transmission was determined from the analog difference of the I1 and I2 signals.

During the experiment, contributions from polarization systematics were minimized by locating the beam along the symmetry-axis of the transmiscion detectors. The beam position, intensity, size, and net transverse polarization ( $\left.T_{\mathrm{pol}}\right)$ were monitored for every pulse. In addition, the transverse-polarization distribution across the beam profile was sampied. To correct the transmission for systematic contributions, the sensitivity to different systematics were determined. Final corrections to the transmission were applied in the off-line analysis. 


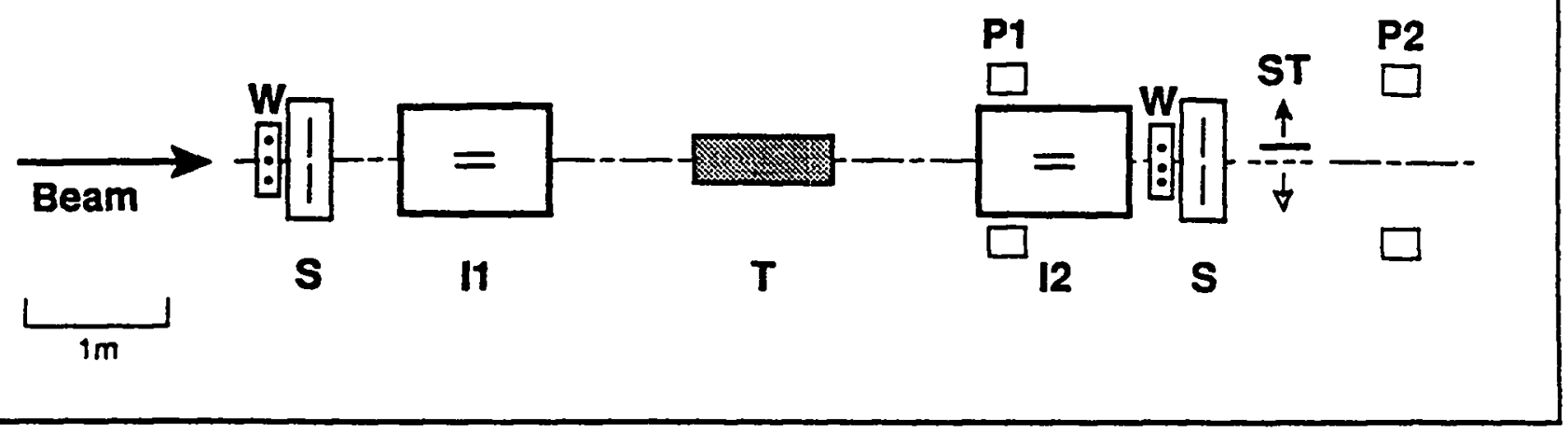

Fig. II-21. Schematic of experimental setup. Detectors are described in the text.

The measured parity-nonconserving longitudinal asymmetry in hydrogen is $A_{L}=(+2.4 \pm 1.1 \pm 0.1) \times 10^{-7}$.

No theoretical approach describes the energy dependence of $p$-nucleon scattering at all energies. The meson-exchange approach can explain experimental results at energies up to $1.5 \mathrm{GeV} / \mathrm{c}$, but underestimates the 6$\mathrm{GeV} / \mathrm{c}$ result. The $\mathrm{QCD}$ approach is consistent with the 1.5- and 6-GeV/c results, but is not applicable at low energies. The existing measurements of $A_{L}$ at $1.5 \mathrm{GeV} / \mathrm{c}$ and $6 \mathrm{GeV} / \mathrm{c}$ indicate a strong energy dependence of the amplitude for the interference between the strong and nonleptonic weak interactions. These experiments were originally envisioned as a study of the weak interaction between nucleons, but the most difficult parts of the problem for theorists are the strong-interaction aspects. The indication that the diquark component of the nucleon is important is very intriguing, but needs to be validated by further experiments.

\section{Study of the Weak Radiative Decay $\pi \rightarrow e v \gamma$}

The radiative decay of the charged pion, $\pi^{+} \rightarrow \mathrm{e}^{+} v y$, is sensitive to strong-interaction corrections when the photon is emitted directly from the weak vertex. In the Standard model, the emission can be associated with either the weak vector or the weak axial-vector currents: the contributions from these currents (the structure-dependent contributions) are characterized by the pion weak form factors, $F V$ and $F_{A}$, respectively. In addition, the amplitude for this decay contains contributions from $Q E D$ corrections to the $\pi^{+} \rightarrow \mathrm{e}^{+} v$ decay (bremsstrahlung).

The vector form factor FV can be uniquely related to the $\pi^{0}$ decay width using the conserved vector current hypothesis. Thus the experimental goal in studies of this decay has been to determine the value of the form factor ratio $\gamma$ where $\gamma=F_{A} / F V$. Experimentally, the problem is to isolate the structure-dependent contributions from the copious, but uninteresting, bremsstrahlung contributions. This was done prior to 1985 by detecting only those events in which the photon and the positron were emitted nearly back-to-back in the pion rest frame, where the bremsstrahlung contribution is very small. From these measurements, one actually determines the quantity $(1+$ $\gamma)^{2}$. resulting in a quadratic ambiguity. The measurements gave $\gamma=0.41 \pm 0.06$ or $\gamma=-2.36 \pm 0.06$.

The Crystal Box at LAMPF was used to detect radiative pion decay events over a larger region of the Dalitz plot, to resolve this ambiguity. Events were obtained with $\theta_{c \gamma}>105^{\circ}$ for somewhat restricted values of the photon and positron energies. A maximum likelihood fit to the distribution of events preferred a positive value for $\gamma$ to a negative value by a factor of 2175:1. The published result is $\gamma=0.25 \pm 0.12$. At nearly the same time, two new results from SIN confirmed the positive solution.

More recently, the radiative pion decay has been used to constrain the parameters of the chiral perturbation theory of QCD. Holstein has shown that many experimental observables can be calculated with only three free parameters. These parameters are constrained by measurements of the pion charge radius, $F_{A}$ from radiative pion decay, and the decay constants of the pion and kaon. The chiral predictions are in good agreement with the remaining observables except for the electric polarizability of the pion, where there is serious disagreement.

A recent experiment studying pion radiative decay in flight measured a total decay rate that disagrees with expectations. This has been interpreted as evidence for the need for a tensor current, which is outside the Standard Model. 


\section{Electroweak Physics}

\section{Precision Tests of the Standard Model}

\section{Introduction}

The Standard Model of Weinberg, Salam, and Glashow describes, at present, all confirmed electroweak phenomena, subject only to computational skill and unknown fitted parameters. Despite this incredible synthesis, much remains to be determined. There are ad hoc assumptions and at least $\mathbf{1 7}$ parameters in the model. For example, the mass spectrum of the basic fermions and the fundamental coupling constants are not explained. In view of this, the challenge is clear. Physicists must seek at a deep level the origin of the mass spectrum and coupling constants, and devise stringent tests to elucidate the model.

LAMPF is an excellent laboratory, with several unique capabilities, to study the Standard Model. The neutrino beam has the highest flux of low-energy neutrinos in the world and affords a very clean and wellunderstood source of $v_{e}$ from muon decay for studying $v_{\mathrm{e}} \mathrm{e}^{-}$elastic scattering. The surface muon beam has a very high flux and is ideal for performing precision tests of the muon, such as measuring the muon magnetic moment and the Michel parameters in muon decay. Finally, the high-quality pion beams at LAMPF allow the study of rare pion decays. The best measurement of pion beta decay and the best sensitivity to some rare $\pi^{0}$ decays come from LAMPF experiments, and further improvements are expected in the future. Below we summarize some of the experiments that have tested (and will continue to test) the Standard Model with precision.

\section{Measurement of the Michel Parameters with the MEGA Positron Spectrometer}

Normal muon decay, $\mu \rightarrow e v \bar{v}$, is the most accessible purely leptonic process and has always been considered an important testing ground for models of the weak interaction. In recent years, sufficient information ahout muon decay has been accumulated to permit a model independent analysis of the interaction, but improved precision is needed to distinguish between the Standard Model of the weak interaction and possible extensions. Any deviation of the Michel parameter $\rho$ from the Standard Model value of 0.75 would indicate new physics. If experiment should reveal departures from the predictions, there are a host of candidate theories providing ready explanations. An example is the left-right symmetric model based on the gauge group $S U(2)_{R} \otimes$ $S U(2) L \otimes U(1)$. In this model, the weak charged current is carried by right- as well as left-handed bosons $\left(W_{R}, W_{L}\right)$, and the Michel parameter $\rho$ becomes $\rho \simeq$ $0.75\left(1-2 \zeta^{2}\right)$, where $\zeta$ is the mixing angle of the chirality eigenstates. An improvement in the precision of $\rho$ by a full factor of ten would be desirable, although a factor of three will significantly restrict the allowed mixing angle (see Fig. III-1).

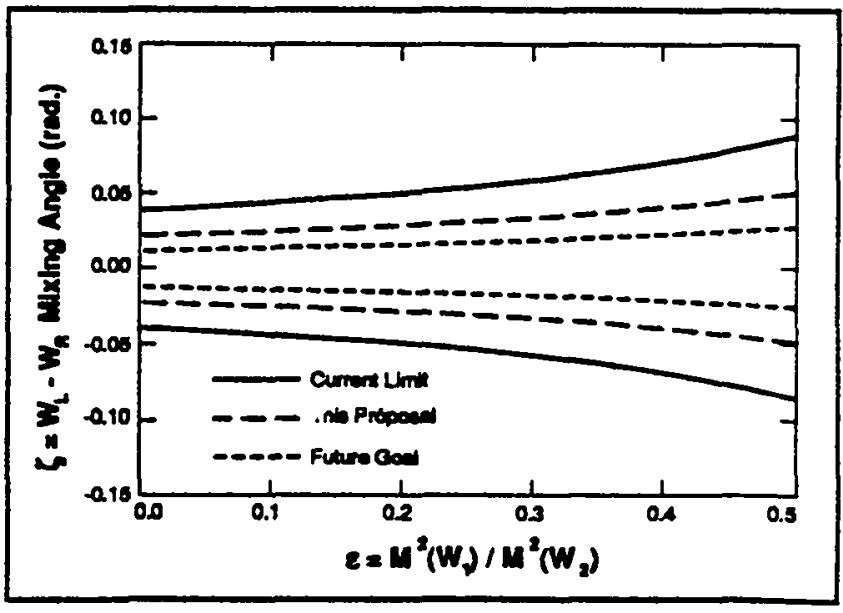

Fig. III-1. The current, proposed, and future limits of the $W_{L}-W_{R}$ mixing angle as a function of $\varepsilon=$ $M^{2}\left(W_{1}\right) / M^{2}\left(W_{2}\right)$. 
An experiment at LAMPF will use the MEGA detector to determine $\rho$ by measuring the energy spectrum of positrons from normal muon decay. The first stage has a goal of $\delta \rho / \rho=0.001$, which is nearly a factor of three improvement in precision from the present best value of $p=0.7518 \pm 0.0026$. Although MEGA was not designed for this measurement, the MEGA apparatus has good acceptance for positrons with $0.75<\mathrm{E}_{\mathrm{J}} / \mathrm{E}_{\max }<1.0$ in a single setting of the magnetic field. The expected statistical precision is $\sqrt{7.5 / N}$, where $N$ is the number of events in this part of the spectrum, and the systematic errors are expected to contribute below the level of $\delta \rho / \rho=$ 0.0002 . Following the completion of the $\mu \rightarrow e \gamma$ experiment, a second phase of the Michel parameter experiment is planned where the precision of $\rho$ will be improved by another factor of three with significant improvements on the precision of several other Michel parameters.

\section{Pion Beta Decay}

The rate of pion beta decay, $\pi^{+} \rightarrow \pi^{0} \mathrm{e}^{+} \mathrm{V}_{\mathrm{e}}$, tests the conserved-vector-current (CVC) hypothesis. CVC, a comerstone of the electroweak theory, directly relates the $f t$ values from nuclear beta decay to the rate of pion beta decay. Current predictions for the rate have only a $0.1 \%$ uncertainty. In addition, pion beta decay provides a measure of the $\Delta S=0$ mixing angle in the CabibboKobayashi-Maskawa matrix, independent of the nuclear beta decay experiments.

Pion beta decay is a rare decay with a branching ratio of about $10^{-8}$. In 1980 , building on the experience from previous experiments, a major attempt to measure pion beta decay was mounted at LAMPF by a Temple/LANL collaboration. Using a 522-MeV/C $\pi^{+}$beam and the $\pi^{0}$ spectrometer, the experiment collected more pion beta decay events than the total previous world sample. Figure III-2 shows the spectrum of the sum of detected photon energies. The peak at $-550 \mathrm{MeV}$ corresponds to pion beta decay events. This is a remarkably clean spectrum, especially considering the fact that the branching ratio for pion beta decay is so small. The final result was

$$
\frac{R\left(\pi^{+} \rightarrow \pi^{0} e^{+} v\right)}{R\left(\pi^{+} \rightarrow \text { all }\right)}=(1.026 \pm 0.039) \times 10^{-8} .
$$

which is a factor of two improvement in precision over the best previous experiment. An auxiliary result from the experiment was a factor of two improvement in the measurement of the $\pi^{-p}$ charge-exchange forward differential cross section at $522 \mathrm{MeV} / \mathrm{c}$.
Future improvements in the -measurement of pion beta decay require better control of systematic errors and a more intense beam. The use of PIIAC would remove both the proton and muon contaminations in the beam, thus reducing a major systematic uncertainty. Recent advances at LAMPF in high-speed data acquisition would allow the event rates needed to gather adequate statistics. A new experimental design would be able to achieve a measurement with an uncertainty of about $0.2 \%$.

Search for the Decays $\pi^{0} \rightarrow v v^{\prime}$ and $\eta \rightarrow v^{\prime}$

The rare decays $\pi^{0} \rightarrow w^{\prime}$ and $\eta \rightarrow w^{\prime}$ are forbidden for massless Weyl neutrinos and can proceed only if neutrino states of both chiralities exist or if lepton number is not conserved. The expected branching ratios for these decays in the Standard Model for reasonable neutrino masses are negligible. However, the observation of a branching ratio larger than $2.8 \times 10^{-9}$ might imply the presence of Higgs contributions with couplings stronger than the standard ones; branching ratios as large as the current limits are possible with not unreasonable values of the Higgs couplings.

The LAMPF experiment 645 and the upcoming LSND experiment are sensitive to the decays $\pi^{0} \rightarrow v v^{\prime}$ and $\eta \rightarrow v v^{\prime}$ vis the reaction $v p \rightarrow v p$, which is independent of neutrino flavor. Because the neutrinos resulting from these decays are much more energetic than neutrinos from pion and muon decay, the recoil protons will have characteristic high energies with very little background. From data already collected, E645 has

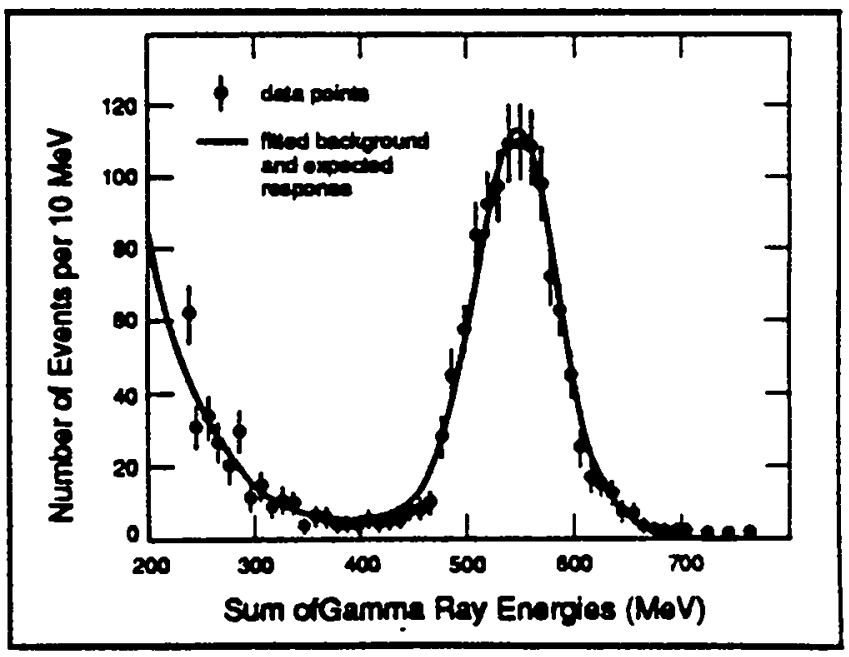

Fig. III-2. The sum of the detected photon energies from the Temple/LANL collaboration. The peak at $-550 \mathrm{MeV}$ corresponds to pion beta decay events, while the lower energy events are background. 
expected sensitivities to these decays of $-10^{-6}$ and $\sim 10^{-5}$, respectively, while the LSND experiment should be an order of magnitude more sensitive.

\section{Search for the C-Noninvariant Decay $\pi^{0} \rightarrow m$}

The Standard Model of electroweak interactions is constructed to be invariant under the operation of charge conjugation $\mathrm{C}$, which reverses the sign of all generalized charges of a system - electric charge, baryon number, lepton number, etc. To date, no evidence for Cnonconservation has been found. However, some of the standard interactions are not invariant under parity reversal, $\mathrm{P}$, or a combined CP-reversal. Thus, it is natural to ask whether the apparent $\mathrm{C}$-invariance is fundamental or simply due to some dynamical suppression or approximate selection rule. One of the cleanest tests for C-noninvariance is the decay $\pi^{n} \rightarrow r \gamma \gamma$, which is manifestly $\mathrm{C}$-noninvariant.

The decay $\pi^{0} \rightarrow \gamma \gamma$ is expected to be suppressed relative to $\pi^{0} \rightarrow \gamma \gamma$ by an extra electromagnetic coupling, phase space, and "centrifugal barrier" effects. Thus, the largest expected value for $\mathrm{B}_{3 \gamma}=\Gamma\left(\pi^{0} \rightarrow \gamma \gamma\right) /$ $\Gamma\left(\pi^{0} \rightarrow \gamma \gamma\right)$ is $-3 \times 10^{-6}$, even if $\mathrm{C}$-invariance is maximally violated.

Two experiments have been performed at LAMPF to search for $\pi^{0} \rightarrow r \gamma$. In the first, a group from Temple University lowered the pre-existing upper limit for the branching ratio by more than an order of magnitude to $\mathrm{B}_{3 \gamma}<3.7 \times 10^{-7}$ (90\% C.L.). Subsequently, the same group joined with the Crystal Box group to lower the upper limit to $\mathrm{B}_{3 \gamma}<3.1 \times 10^{-8}$ (901\% C.L.). This remains the best limit for this decay and indicates that $C$ invariance is not maximally violated in this process.

\section{Muonium and Muonic Atoms}

Muonium experiments performed at LAMPF by the Yale-Heidelberg-Syracuse collaboration are providing successively more stringent tests of quantum electrodynamics (QED) and the Standard Model. These experiments determine the muonium hyperfine structure interval $\Delta v$ and the magnetic moment ratio $\mu_{\mu} / \mu_{\mathrm{p}}$ by measuring hyperfine Zeeman transitions in a strong magnetic field. While the determination of $\mu_{\mu} / \mu_{p}$ is important in its own right as a fundamental measurement, its value is needed to exploit the upcoming measurement of $\left(g_{\mu}-2\right)$ at Brookhaven as a sensitive test of quantum electrodynamics.

The muonium atoms are stopped in a low-density gas target located in an RF cavity. The RF frequency is held fixed while the magnetic field is scanned through the resonance, which appears as a dip in the positron frontback asymmetry. At present, $\Delta v$ is determined by the LAMPF experiment to $0.036 \mathrm{ppm}$ and $\mu_{\mu} / \mu_{\mathrm{p}}$ to 0.36 ppm.

A new ultra-high precision measurement experiment is being assembled to determine $\Delta v$ to $10 \mathrm{ppb}$ and $\mu_{\mu} / \mu_{\mathrm{p}}$ to $50 \mathrm{ppb}$, which corresponds to improvements of from 5 to 10 times the present values. The experiment employs magnetic resonance spectroscopy on intense and pure subsurface $\mu^{+}$beams created in the stopped muon channel. The ultra-high precision will be obtained through experimental innovations such as the use of a large homogeneous solenoidal magnetic field and line-narrowing methods using chopped $\mu^{+}$beams. The chopped beams will allow the restriction of the asymmetry measurements to "old muonium," with a consequent narrowing of the resonance curves. Even more significant improvements in precision are possible with the proposed pulsed lepton source (PLS), since pulsed beams will allow the observation of the muon asymmetry as a function of time with no sacrifice in statistics.

Additional experiments such as the $2 \mathrm{~s}$-Lamb shift measurements on muonium, the search for muonium to antimuonium conversion, and the development of polarized muonic atoms are providing more stringent tests and constraints on the Standard Model. The latter two experiments are discussed elsewhere.

\section{E225: Neutrino Electron Scattering and Neutrino Properties}

The LAMPF beam stop is a high-intensity laboratory source of $v_{\mu}, \bar{v}_{\mu}$, and $v_{e}$ neutrinos unmatched in the world. Using this unique source of $v_{e}$, LAMPF experiment E225 has performed the first and only observation of the purely leptonic process $v_{e} e^{-}$- elastic scattering.

Neutrino-electron scattering is a simple and fundamental process with great sensitivity to fundamental details of the Standard Model, including dynamic properties of the weak interaction, such as the weak mixing angle $\sin ^{2} \theta \mathrm{w}$ and the interference between charged and neutral current amplitudes, and static properties of the neutrino, such as electromagnetic moments and neutrino decay. It is a purely weak, purely leptonic two-body reaction, which makes both the theoretical calculations and the experimental signature straightforward. E225 measured the absolute cross section for $v_{\mathrm{e}} \mathrm{e}^{-}$elastic scattering, which determines the interference term between $W^{ \pm}$and $Z^{0}$ exchange as tests of the Standard Model. In addition, new limits on several neutrino properties that are not contained in the minimal Standard Model have been 
set. A new program that would be possible with a Pulsed-Lepton-Source facility (PLS) using imaging Cerenkov-scintillator detectors would greatly increase the precision of these tests.

E225 employed a fine-grained, 15-ton, tracking detector to measure the energy and track direction of the recoil electrons. The electron from $v^{-}$elastic scattering recoils along the incident neutrino direction with an angle less than $16^{\circ}$, whereas backgrounds from both cosmic rays and accelerator neutrons are essentially isotropic. The signal is then apparent in the recoil angular distribution as a prenounced peak in the forward direction. The experimentally observed angular distribution, and clear $\mathrm{ve}^{-}$elastic scattering peak, is shown in Fig. II-3.

The rate for $v_{e} e^{-}$scattering is obtained by subtracting the contributions from $\left(v_{\mu}+\bar{v}_{\mu}\right) e^{-}$events, and yields an absolute cross section, for a mean energy $\left\langle E v_{c}\right\rangle=31.7$ $\mathrm{MeV}$, of $\sigma\left(V_{\mathrm{e}} \mathrm{e}^{-}\right) / \mathrm{E}_{\mathrm{V}}=(10.0 \pm 1.5 \pm 0.9) \times 10^{-45}$ $\mathrm{cm}^{2} / \mathrm{MeV}$. The interference term, $\sigma$, is measured directly, and in a model independent manner, by the difference between the measured elastic scattering total cross section and the sum of the charged current and neutral current contributions, $\sigma C C$ and $\sigma^{N C}$, respectively. Thus, $\sigma^{I}=\sigma^{\text {tot }}-\sigma^{C C}-\sigma^{N C}$, where $\sigma^{C C}$ and $\sigma^{N C}$ have been measured in muon decay and in $v_{\mu} \mathrm{e}^{-} \rightarrow v_{\mu} \mathrm{e}^{-}$ scattering. For $\sin ^{2} \theta w=0.23$, the agreement between experiment and the Standard Model is excellent.

It is the comparison of the measured $v_{e} e^{-}$strength to that predicted by the electroweak Standard Model that places limits on nonstandard physics.

A neutrino magnetic moment would produce an excess of elastic scattering events. A fit to the

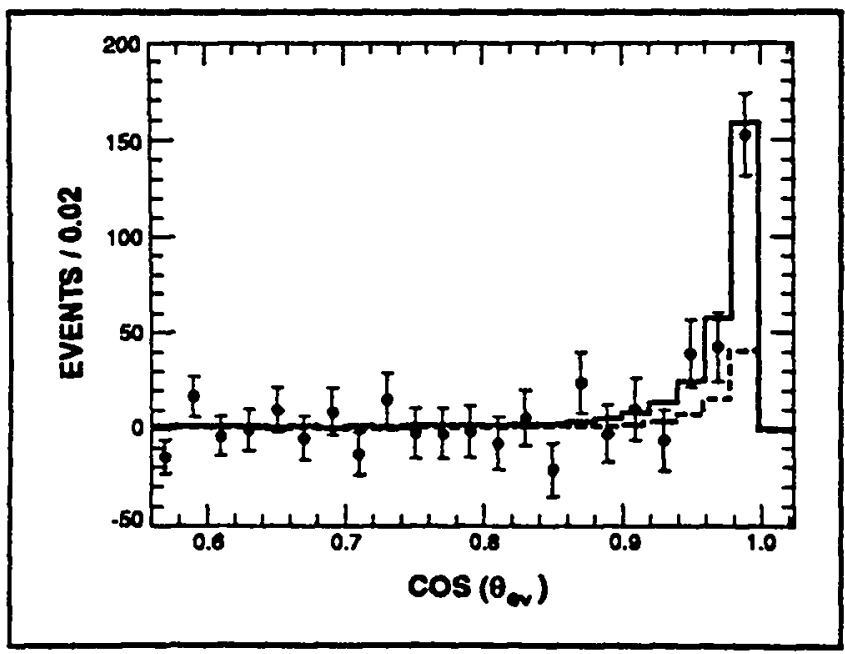

Fig. III-3. Angular distribution of the measured elastic scattering signal. The solid line is the result of the best fit, $295 \pm 35$ events. The dotted line is the background contribution from the $60\left(v_{\mu}+\bar{v}_{\mu}\right) e^{-}$scattering events. experimental angular distribution yields $90 \%$ confidence limits on the neutrino magnetic moments of $\mu_{v_{e}} \leq$ $10.6 \times 10^{-10} \mu_{\text {Bohr }}$ and $\mu_{v_{\mu}} \leq 7.4 \times 10^{-10} \mu_{\text {Bohs }}$. The upper limit obtained for for $\mu_{v_{e}}$ is consistent with that for $\bar{\mu}_{v_{e}}$ from a reactor experiment, while the bounds on $\mu_{v_{\mu}}$ are more resurictive than previous results.

A neutrino charge radius is not a gauge invariant quantity. Nevertheless, a measurable neutrino chargeradius form factor can be defined for low $q^{2}$ laboratory interactions as an additive correction to the effective weak neutral-current vector coupling, gV. A nonzero charge radius shifts $\mathrm{gV}$ to $\mathrm{gV}=-\frac{1}{2}+2\left(\sin ^{2} \theta w+\delta\right)$, where $\sin ^{2} \theta w$ is the weak mixing angle measured in nonneutrino interactions and $\delta=\left(\sqrt{2 \pi \alpha / 3 G_{F}}\right)\left\langle r^{2}\right\rangle=2.39 \times$ $10^{30} \mathrm{~cm}^{-2}$. The data from the LAMPF experiment implies $-0.170<2 \delta<0.260$, so that $-3.56 \times 10^{-32}$ $\mathrm{cm}^{2}<\left\langle\mathrm{r}^{2}\right\rangle<5.44 \times 10^{-32} \mathrm{~cm}^{2}$ (90\% C.L.). Thus, the $v_{e}$ charge radius is $|r|<2.2 \times 10^{-16}$ with $90 \%$ confidence, which represents a new upper bound on the dimensions of internal structure of the electron neutrino.

Consideration of weak neutral currents has been central to the development of the standard model of electroweak interactions. Much of the formal structure of the standard model derives from the necessity to eliminate flavor-changing neutral currents (FCNC) at the tree-level since such currents have not been observed experimentally. The interference effect in $v_{e} e^{-}$scattering is not present if the outgoing neutrino is not a $v_{e}$; thus $v_{\mathrm{e}} \mathrm{e}^{-}$provides a sensitive probe of FCNCs. Purely phenomenological couplings, $f_{e e}, f_{e \mu}$, and $f_{e \tau}$, can be introduced for the three neutrino families; the standard model is recovered by setting $f_{e \mu}=f_{e \tau}=0$. Explicit limits from this experiment give the mean value $\mathrm{f}_{e e}=$ 0.93 , and the $90 \%$ confidence limit for an off-diagonal, flavor-changing coupling, as $1-f_{e e}<0.35$. Alternatively, through use of the normalization relation, we have a limit on the total strength of flavor-changing transitions of $\mathrm{f}_{\mu}^{2}+\mathrm{f}_{\mathrm{e \tau}}^{2}<0.58$ (90\% C.L.).

It is not known if the neutrino is absolutely stable, and if not, by what modes it would decay. Neutrino decay requires lepton flavor violation and the existence of at least one massive neutrino species. The electromagnetic decay, $v \rightarrow \gamma+X$, is especially interesting because it can be related to the intemal structure of the neutrino. A search has been made for this decer' mode in an appearance experiment, by detecting $y$ rays within the $31.5 \mathrm{~m}^{3}$ E225 detector. The E225 experiment is sensitive to gamma rays in the energy range $15 \mathrm{MeV}<\mathrm{E} \gamma<53 \mathrm{MeV}$. The data are consistent with no excess $\gamma$ rays. Under the assumption of equal radiative lifetime for $v_{\mu}$ and $\bar{v}_{\mu}$, limits obtained for each may be combined to yield $\tau / m_{v_{\mu}}>15.4 \mathrm{sec} / \mathrm{eV}$. 
Finally, limits can be placed on the cross section for exchange of scalar, vector or tensor bosons that are not included in the Standard Model. The observed number of $v_{\mathrm{e}} \mathrm{e}^{-}$elastic scattering events, $295 \pm 35$, is to be compared to the Standard Model prediction of $284 \pm 26$. Therefore, at $90 \%$ confidence level, anomalous (beyondthe-SM) interactions contribute fewer than 78 events. If terms proportional to $m_{e} / E_{v}$ are ignored, then the differential cross section for any interaction composed of the sum of $S, P, T, V, A$ components can be expressed as $d \sigma / d y \propto A+B(1-y)+C(1-y)^{2}$ and the total cross section is simply $\sigma=(A+B / 2+C / 3) \sigma_{0}$, where $\sigma_{0}=$ $\mathrm{G}_{\mathrm{F}}^{2} \mathrm{~m}_{\mathrm{e}} \mathrm{E}_{\mathrm{v}} / 2 \pi$. For couplings of the same strength as the weak charged or neutral current, we can extract general limits on the masses for new bosons, as listed in Table III-1. In general, limits obtained here on the mass of hypothetical bosons are similar to limits obtained by direct searches for such bosons in collider experiments. However, limits from neutrino-electron scattering would be important for ruling out particular extensions of the Standard Model that involve bosons that couple mainly, or only, to leptons, or weak interactions that only couple fermions within the same weak isodoublet.

Table III-1. Limits on new gauge boson masses for S, P, $T, V$, and A couplings.

\begin{tabular}{|llr|}
\hline Coupling & Charge & Mass Limit \\
\hline T & Neutral & $105 \mathrm{GeV} / \mathrm{c}^{2}$ \\
S, P & Neutral & $47 \mathrm{GeV} / \mathrm{c}^{2}$ \\
Higgs (S) & Charged & $87 \mathrm{GeV} / \mathrm{c}^{2}$ \\
Left-Handed (V,A) & Charged/Neutral & $230 \mathrm{GeV} / \mathrm{c}^{2}$ \\
\hline
\end{tabular}

\section{The Family Number Problem}

\section{Introduction}

The existence of the muon, which appears to be a heavy electron, is a central problem in particle physics. The absence of the process $\mu^{+} \rightarrow \mathrm{e}^{+} \gamma$ and the three-body character of the muon decay spectrum led to the postulation of separate mion and electron neutrinos that was later experimentally confirmed. In a modern context, many empirical facts about the elementary particles have been catalogued in an elegant periodic table called the minimal Standard Model. The Standard Model, which otherwise describes the interactions among the elementary particles, does not explain the repetition of the particle types; the groupings are known as families.
The Standard Model has been too successful to be wrong, but it most likely appears incomplete because of its large number of ad hoc parameters. The Standard Model is, in reality, a reduction of some more general picture. The origin of the families cries out for explanation. In the lepton sector of the minimal Standard Model, there is no mixing between the families at all. There is no motivation for the neutrinos to be massless, no reason for the families not to decay into one another, and no known symmetry principal to keep them apart.

The unique high-intensity beams available at LAMPF are essential to studies of the family problem. Neutrino experiments and rare decays directly address the specific questions, "Are neutrinos massive, is there mixing, and can family transitions be observed?" The weak interaction of the neutrino with detectors requires the high flux from LAMPF. The sensitivity needed to probe for inter-family transitions or search for new particles makes the examination of an enormous number of decays essential.

\section{Character of the Conservation Law}

Conservation of family number is a purely empirical law and may be formulated in a variety of ways. The additive form conserves the number of particle of a particular type, e.g., the number of muon-like particles by allowing weak decays from muons to muon neutrinos. The multiplicative form conserves the total number of leptons and their "family parity." There are even models (left-right symmetry) that tolerate a mixture of the two forms. An example of a reaction that satisfies the multiplicative form but violates the additive form is the reaction $\mu^{+} \rightarrow \mathrm{e}^{+} \bar{v}_{\mathrm{e}} v_{\mu}$, where the "wrong" neutrinos are emitted.

In 1980, LAMPF E31 published the first limit on the branching ratio $R=\Gamma\left(\mu^{+} \rightarrow e^{+} \bar{v}_{e} v_{\mu}\right) / \Gamma\left(\mu^{+} \rightarrow\right.$ all $)<$ 0.098 (90\% confidence). The CHARM collabora-tion at CERN improved the limit to 0.05 , and recently an experimental search was performed at the LAMPF beam stop as part of an electron-neutrino scattering experiment (\#225). The appearance of electron antineutrinos arising from the exotic decay mode would be signaled by inversebeta decay of protons, $\bar{v}_{e}+p \rightarrow e^{+}+n$, in a hydrogenrich $3 \times 3 \times 3.5 \mathrm{~m}^{3}$ 15-ton tracking calorimeter. The measured "visible energy" distribution of the final event sample is shown in Fig. III-4. The "visible energy" $E_{e}$ recorded in the scintillators is approximately $60 \%$ of the total energy deposited in the detector. The light solid line in Fig. III-4 represents the contribution expected from exotic muon decay for the branching fraction, $R=0.05$, corresponding to the previous limit on this mode; the heavy solid line is the best fit obtained assuming $R=0$. 


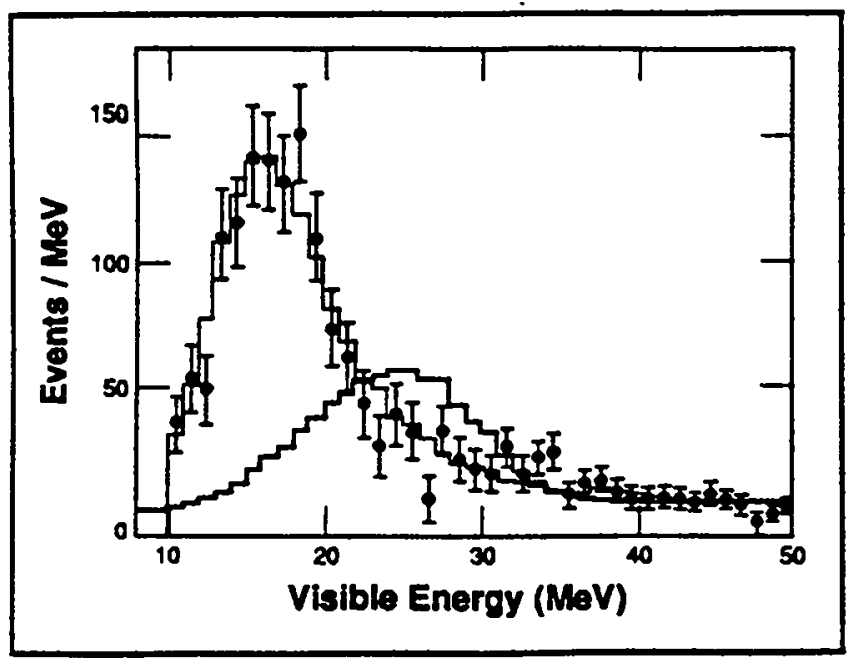

Fig. III-4. The "visible" energy spectrum of the final data sample. The heavy line is the result of the best fit obtained assuming $R=0$. The expected contribution from only $\bar{v}_{e}+p \rightarrow e^{+}+n$ for $R=0.05$ is shown as the lightsolid histogram.

Most of the sensitivity to the presence of $\bar{v}_{e}$ is in this distribution. A fit to the correlated $\left(E_{e}, \theta_{e}\right)$ spectra implies with $90 \%$ confidence that there are fewer than $256 \bar{v}_{e} p$ events observed, leading to a new limit of $R<0.018$. It should be noted that neutrino oscillations through the $\bar{v}_{\mu} \rightarrow \bar{v}_{e}$ mode could also lead to the appearance of $\bar{v}_{e}$ interactions, so that the limit on the inverse-beta decay events also implies limits on neutrino oscillations that do not exclude any new regions of the oscillation parameter space. The LSND experiment, described below, is expected to be sensitive to this decay mode at a level of $R=1.4 \times 10^{-4}$.

\section{Mixing in the Lepton Sector}

The lepton mixing angles and neutrino masses have only experimental upper limits and are often assumed to be zero. By contrast, the quark masses and mixing angles are mostly well determined and can be conveniently expressed in terms of the Cabibbo-Kobayashi-Maskawa mixing matrix. Working by analogy to the quark sector, it is reasonable to believe that the neutrino masses and mixing angles, although small, are non-zero and that neutrino oscillations occur. This view is reinforced by solar neutrino experiments, which strongly suggest that $v_{e}$ from the sun are transformed before reaching the earth. As the question of neutrino masses strongly arfects not only nuclear physics but also particle and astrophysics, it is of utmost importance to search for neutrino masses by all possible means.
LAMPF is an ideal and unique accelerator for searching for neutrino oscillations due to its high intensity, which probes small mixing angles, and low energy, which provides sensitivity at low values of neutrino masses. The oscillation probability is given by $P_{v_{1}} \rightarrow v_{2}=\sin ^{2} 2 \alpha \sin ^{2}\left[1.27 \Delta m^{2} L / E_{v}\right]$, where $\alpha$ is the mixing angle, $\Delta m^{2}=m_{v_{1}}^{2}-m_{v_{2}}^{2}$ in $e^{2}, L$ is the neutrino propagation distance in meters, and $E_{V}$ is the neutrino energy in MeV. Figure III-5 shows the present $v_{\mu} \rightarrow v_{e}$ oscillation limits from reactor (Gosgen), BNL (E734), and LAMPF (E645,E764) experiments in the $\Delta \mathrm{m}^{2}, \sin ^{2} 2 \alpha$ parameter space.

Also shown in Fig. III-5 are the limiting curves expected from the $v_{\mu} \rightarrow v_{e}$ and $\bar{v}_{\mu} \rightarrow \bar{v}_{e}$ measurements of the Liquid Scintillator Neutrino Detector (LSND) experiment, which will use neutrinos from $\pi^{+}$decay in flight and $\mu^{+}$decay at rest, respectively. The two measurements, carried out at the same time, in the same beam(s), and with the same detector will provide independent checks on the validity of the final result on neutrino oscillations not available from previous measurements. After two years of data collection, 90\% confidence level limits on $\bar{v}_{\mu}\left(v_{\mu}\right) \rightarrow \bar{v}_{e}\left(v_{e}\right)$ mixing strengths of $2.7(2.7) \times 10^{-4}$ can be obtained for all $\Delta \mathrm{m}^{2}>1 \mathrm{eV}^{2}$. This region is especially interesting from a cosmological standpoint because a long-lived neutrino species with mass $\sim 1 \mathrm{eV}$ would contribute as much mass to the universe as from the visible matter, while a $\sim 30-\mathrm{eV}$ neutrino mass would close the universe.

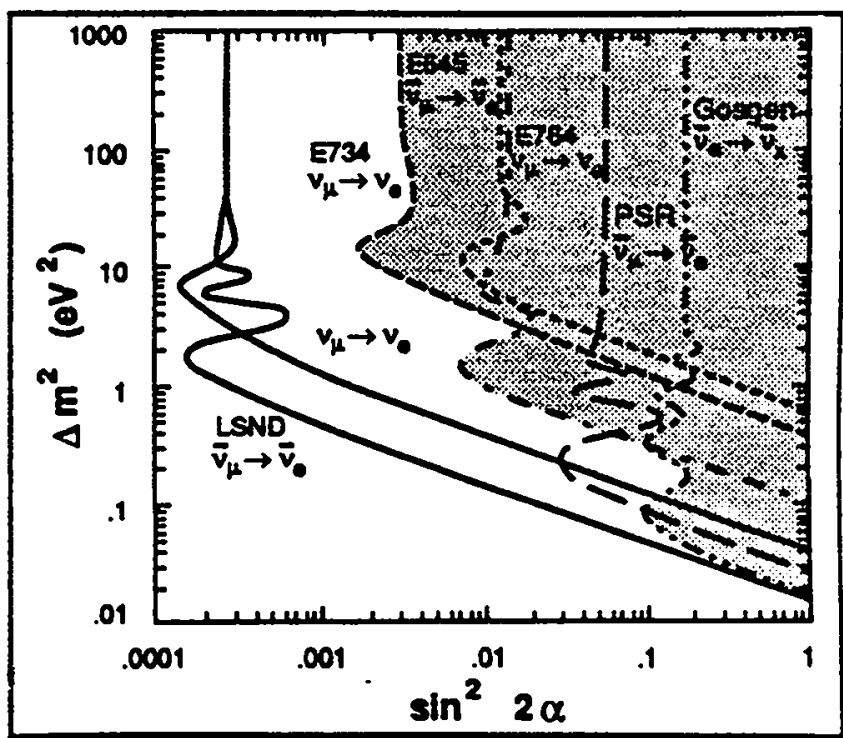

Fig. III-5. Existing limits for $v_{\mu} \rightarrow v_{e}$ and $\bar{v}_{\mu} \rightarrow-v_{e}$ oscillations from a variety of experiments and the expected limits from LSND. The limits are in the $\Delta m^{2}, \sin ^{2} 2 \alpha$ parameter space. The excluded regions are above and to the right of the curves. 
The LSND experiment will also be sensitive to $v_{\mu} \rightarrow v_{\tau}$ oscillations in two different ways. First, a search for $v_{\mu} \rightarrow v_{e}$ includes the second order process $v_{\mu} \rightarrow v_{\tau} \rightarrow v_{e}$, where $\Delta m^{2} \sim m_{v_{\tau}}^{2}$ for large $\mu_{v_{\tau}}$ and the mixing probability is equal to $\left(\sin ^{2} 2 \alpha_{v_{\mu}} \rightarrow v_{\tau}\right) \times$ $\left(\sin ^{2} 2 \alpha_{v_{\tau}} \rightarrow v_{e}\right)$. Second, the experiment will be able to search for $v_{\mu}$ disappearance to the $10^{-2}$ level in the region $4<\Delta \mathrm{m}^{2}<400 \mathrm{eV}^{2}$ by looking for a variation in the $v_{\mu} \mathrm{C} \rightarrow \mu-\mathrm{X}$ event rate as a function of distance along the beam within the detector.

The detector, shown in Fig. III-6, consists of a cylindrical tank of dilute mineral-oil-based liquid scintillator such that about $20 \%$ of the total light output will be Cerenkov light and $80 \%$ scintillation light. There will be 1224 8" diameter, low time-jitter photomultiplier tubes mounted uniformly over the inside tank surface
(25\% coverage). These tubes are manufactured by Hammamatsu and have excellent timing resolution (3.8-ns FWHM for single photons and full-face illumination) and single photoelectron separation. The tank is approximately $6 \mathrm{~m}$ in diameter by $9 \mathrm{~m}$ long with an active mass of 200 tons and will reside inside the existing E645 veto shield, which is located $27 \mathrm{~m}$ downstream of the proton beam stop and is at an angle of approximately $12^{\circ}$ to the beam direction. It has been proposed that the beam stop be upgraded by increasing the pion decay-in-flight path and by installing a magnet to bend the proton beam toward the detector. Such an upgrade would increase the decay-inflight $v_{\mu}$ flux by a factor of five and thereby improve both the $v_{\mu} \rightarrow v_{e}$ and $v_{\mu}$ disappearance oscillation searches by roughly a factor of 2 .
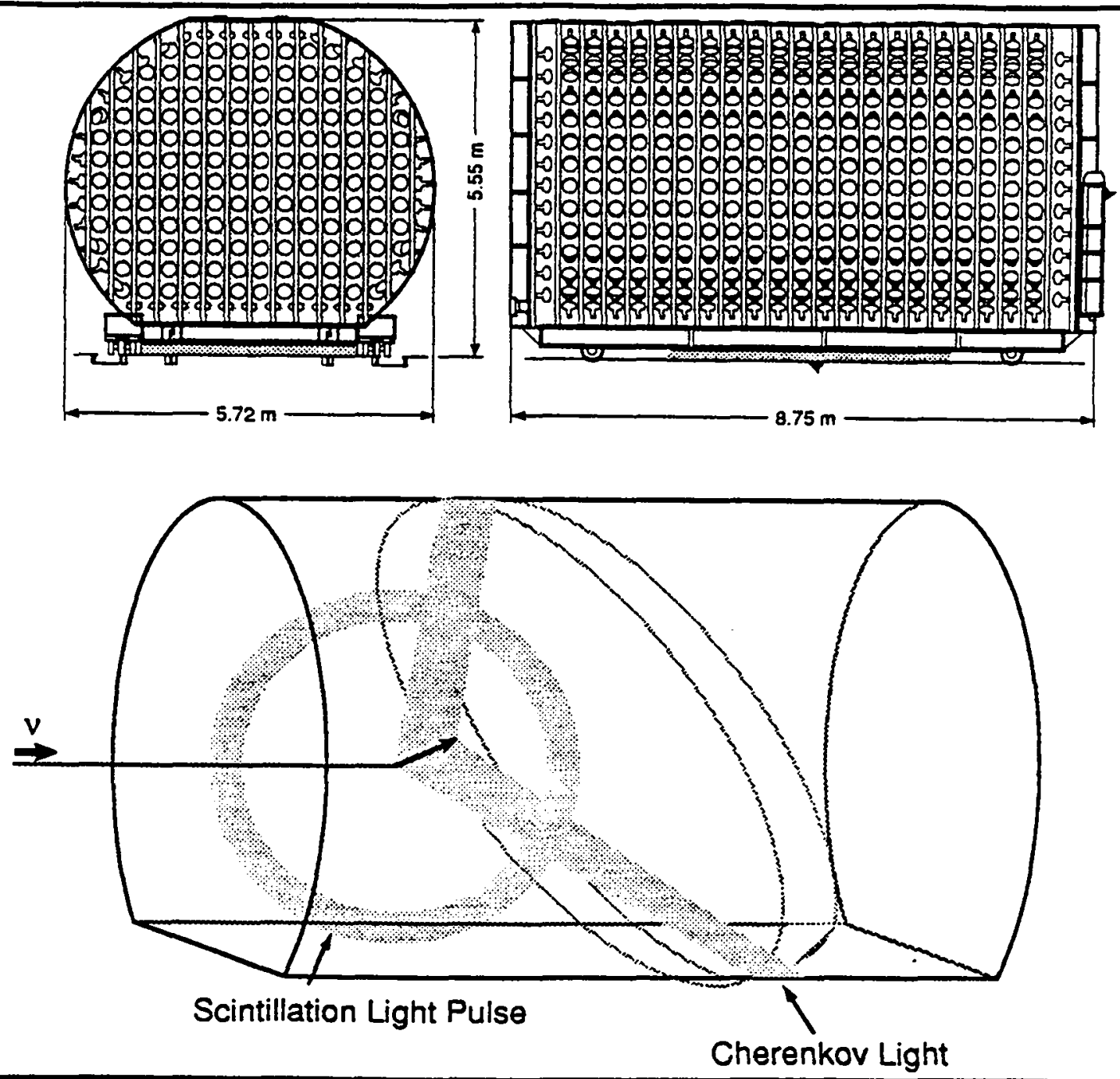

Fig. III-6. A schematic view of the LSND detector, consisting of a 6-m-diameter by 9-m-long cylindrical tank of dilute liquid scintillator with 1224 8" diameter photomultiplier tubes covering about 28\% of the surface area of the tank. A cartoon of an event is shown. 


\section{Family Transitions}

Direct transitions between lepton families would give important evidence regarding the distinctions between the families. In principle, if neutrino oscillations were established, family-changing transitions between charged leptons would occur via the creation of virtual neutrinos that mixed to change flavors. However, current oscillation limits make those family transitions unobservably small. Hence, the observation of any direct family transition represents a major extension of the Standard Model, e.g., requiring the existence of new massive particles to mediate the process. Having massive particles mediate a process is familiar in the case of $\mathrm{W}$ bosons mediating beta decay. Generally, a larger mass for the mediating particle leads to a smaller branching ratio for the process. These searches are analogous to attempting to discover beta decay because you suspect the existence of the intermediate vector bosons. Theoreticians, in their attempts to discover the origins of the Standard Model, have given plenty of possibilities for these new particles.

Experiments to search for direct transitions, called rare decays because none have ever been seen, are usually reported in terms of the branching ratio sensitivity for the rare decay to the sum of the allowed decays. Improvements in the sensitivity raise the mass limits as the one-fourth power. Even though this relationship is unfavorable, experimentalists have continued to make such rapid improvement in sensitivity that they have kept pace with the rise in the mass sensitivity from direct production in colliding beam accelerators. Furthermore, the mass range being probed is quite interesting, covering from $50 \mathrm{GeV}$ as far up as hundreds of $\mathrm{TeV}$, depending on the model. Rare decays continue to provide very stringent constraints for model builders.

A large number of rare decay experiments are possible, and theoreticians have invented a large number of possible extensions to the Standard Model. Different experiments turn out to be most sensitive to different categories of models. Thus, it is very important to search for direct family transitions wherever they are possible. If one or more can be found, the relative rates will be strong clues to the underlying mechanism. LAMPF is a leader in the worldwide community that coeks to find interfamily transitions, and a few of the LAMPF experiments are described below

Looking for the rare decay $\mu^{+} \rightarrow \mathrm{e}^{+} \gamma$ is particularly interesting because in has a clean signature and involves only leptons and photons. The signature from decay at rest is purely kinematical and does not depend on the character of the inducing interaction; it is the back-to-back coincidence of a $52.8-\mathrm{MeV}$ positron with a $52.8 \mathrm{MeV}$ photon. Shortly after the rumors that $\mu^{+} \rightarrow \mathrm{e}^{+} \gamma$ had been observed, LAMPF undertook a search for the process; a $90 \%$-confidence limit of $1.7 \times 10^{-10}$ was set, ruling out the earlier possibility. The present world limit for $\mu^{+} \rightarrow$ $\mathrm{e}^{+} \gamma$ comes from the follow-on experiment, the Crystal Box. It involved a large $\mathrm{NaI}(\mathrm{TI})$ calorimeter surrounding a cylindrical drift chamber that tracked the positrons. That limit stands at $4.9 \times 10^{-11}$. Simultaneously, a limit was also set on the process $\mu^{+} \rightarrow \mathrm{e}^{+} \gamma \gamma$ of $7.2 \times 10^{-11}$; this decay mode is favored in certain models, e.g., involving doubly charged leptons, where the GIM mechanism suppresses $\mu^{+} \rightarrow \mathrm{e}^{+} \gamma$.

The new search for $\mu^{+} \rightarrow \mathrm{e}^{+} \gamma$ at LAMPF, called MEGA, an acronym standing for Muon decays into an Electron and a GAmma ray, is an experiment with a branching-ratio sensitivity of roughly $10^{-13}$. The MEGA apparatus is shown in Fig. III-7. The apparatus is contained in a large solenoidal magnet with a clear bore of $1.85 \mathrm{~m}$. The required muon stopping rate is $3 \times 10^{7} \mathrm{~Hz}$ (average). The detector is divided into a positron spectrometer and a series of photon pair spectrometers. All of the charged particles arising from muon decay are confined by the magnetic field to the central region, leaving the photon detectors in a relatively quiet environment.

The positron arm consists of two parts, scintillators for timing and a set of multi-wire proportional chambers (MWPC) for momentum determination. There is a central, cylindrical chamber surrounded by seven small chambers to provide the necessary redundancy for pattem recognition. The photon arm is a series of concentric pair-spectrometers. Each pair spectrometer is made of lead converters, MWPCs, drift chambers for tracking, and scintillators for timing. The drift chambers are outfitted with cathodes that are highly folded delay lines to give the longitudinal momentum components.

Engineering runs have demonstrated the feasibility of the MEGA detector performance. Enough data have been taken searching for the $\mu^{+} \rightarrow e^{+} \gamma$ process to debug preliminary analysis routines and to yield a result that indicates the design is well conceived.

In the next year, the $\mu^{+} \rightarrow \mathrm{e}^{+} \gamma$ search will reach a sensitivity around $5 \times 10^{-12}$. Final data taking is expected in 1993 and 1994 that will make another factor of ten improvement in sensitivity. This detector, with its fine resolutions and high rate capability, portends many other exciting experiments in future years such as improved measurements of Michel parameters for normal muon decay, the muon lifetime, and the $\pi^{0} \rightarrow \mathrm{e}^{+} \mathrm{e}^{-}$ reaction. 


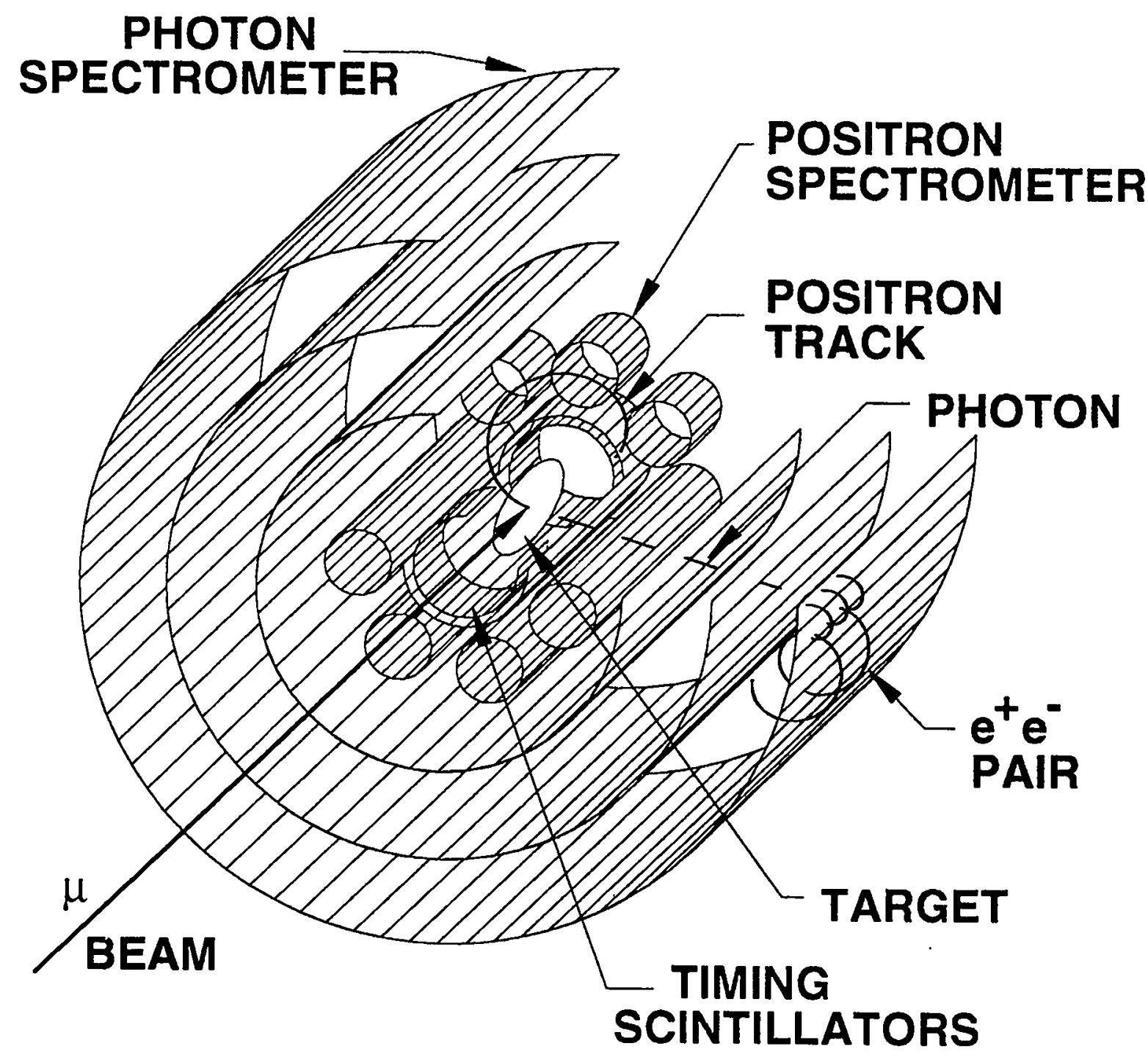

Fig. III-7. A simplified cutaway view of the MEGA apparatus. The detector is mounted inside a superconducting solenoid. The muons enter parallel to the magnetic field and stop in the extended target. Positrons from muon decays are detected in the eight cylindrical wire chambers and the cylindrical arrays of scintillators. The large cylinders are pair spectrometers for photon detection. An idealized signal event is overlaid on the detector. 
The Brookhaven E791 detector searched for the $\mathrm{K}_{\mathrm{L}}^{0} \rightarrow$ $\mu e$ process using a two-arm, two-magnet spectrometer instrumented with drift chambers for charged track reconstruction, a lead glass calorimeter for electron energy measurement, and a muon range finder instrumented with a 6,000-wire drift tube array. The experiment was optimized for the detection of the dilepton decay modes $(e e, \mu \mu, \mu e)$ of the $K_{L}^{0}$. Los Alamos contributed the construction of the muon range stack, the range finder electronics, and custom mean timer electronics for use in the scintillator trigger. E791 obtained the world limits on the branching fraction for $\mathrm{K}_{\mathrm{L}}^{0} \rightarrow \mu \mathrm{e}$ and to $\mathrm{K}_{\mathrm{L}}^{0} \rightarrow$ ce $\left(3.3 \times 10^{-11}\right.$ and $4.1 \times 10^{-11}$, respectively, at the $90 \%$ confidence level) and the largest sample of $\mathrm{K}_{\mathrm{L}}^{0} \rightarrow \mu \mu$ $(\sim 740)$ events, leading to a branching fraction measurement of $7.0 \pm 0.5 \times 10^{-9}$.

Spontaneous conversion of muonium $\left(M=\mu^{+} e^{-}\right)$ into antimuonium $\left(\overline{\mathrm{M}}=\mu^{-} \mathrm{e}^{+}\right)$is forbidden in the Standard Model in which lepton family numbers are additively conserved for each family. However, in various extensions to the Standard Model, $(M-\bar{M})$ transitions can occur. In left-right symmetric theories, in which parity violation is a low-energy consequence of the difference in masses between the $W_{L}$, which governs left-handed interactions, and $W_{R},(M-\bar{M})$ transitions could be mediated either by the exchange of Majorana neutrinos or by doubly-charged Higgs bosons. (M- $\bar{M})$ transitions are characterized by a coupling strength $G_{M} \bar{M}$, which is usually expressed relative to $G_{F}$, the Fermi coupling constant.

There have been two experiments at LAMPF searching for $(M-\bar{M})$ transitions. The first utilized fast muonium produced in a thin foil and the Crystal Box to detect the X-rays that would be emitted when the $\mu^{-}$from $\bar{M}$ is captured in a bismuth target. The result was $G_{M \bar{M}}<7.5 G_{F}$. A more recent experiment utilized the production of thermal muonium in vacuum and a novel detector that observed the $e^{-}$from the Michel decay of the $\mu^{-}$in a magnetic spectrometer, and the atomic $e^{+}$left after the $\mu^{-}$in the $\bar{M}$ has decayed. When the polarities of the detector elements were reversed to check the method, the atomic electron in muonium was seen for the first time. The technique resulted in a large increase in sensitivity.
No (M- $\overline{\mathrm{M}})$ transitions were observed with a resulting $90 \%$ confidence-level upper limit of $G_{M} \bar{M}<0.61 G_{F}$. This is the most sensitive limit for the coupling constant for this process. This result places severe constraints on several left-right symmetric models.

\section{New Particles}

Several light scalar or pseudoscalar bosons, such as familons, axions, and Majorons, have been proposed to solve problems in particle physics. For example, the familon is a Goldstone boson that would arise if the family symmetry is dynamically broken; $1 / F$ is a parameter used to characterize the scale of the symmetry breaking. A number of processes, such as $\mathrm{K}^{+} \rightarrow \pi^{+} \mathrm{f}$ and $\mu^{+} \rightarrow \mathrm{e}^{+} \mathrm{f}$ (where $\mathrm{f}$ is the light boson), can be used to search for these light bosons. The radiative decay mode $\mu^{+} \rightarrow e^{+} \gamma f$ has the advantage that it is independent of the chirality properties of the boson coupling. An upper limit on the branching ratio for the radiative decay mode was obtained in the Crystal Box Experiment. From this value, limits on the scale of symmetry breaking were obtained for several Lagrangians that could characterize the light boson couplings. The scale of the familon symmetry breaking is found to be $>2.9 \times 10^{9} \mathrm{GeV}$. Another implication of the experimental result is that the mass scale for any internal compositeness of the leptons is given by $\mathrm{M}>1.2 \times 10^{4} \mathrm{GeV}$.

\section{Conclusion}

The experimental program at LAMPF to address the family problem, including neutrino and rare decay experiments, has placed stringent constraints on possible extensions to the Standard Model. However, this important problem has not been solved and experiments under way will make important improvements over previous results. Even if physics beyond the Standard Model is not discovered, the theoretical possibilities will be further restricted. In addition, a program of other precision experiments utilizing these sophisticated detectors is planned. 


\section{Additional Basic and Applied Research}

\section{Introduction}

In this section, we describe several different programs, both fundamental and applied in nature, covering such diverse fields as biology, biomedical technology, materials science and technology, atomic and molecular physics, energy technology and nuclear physics. The common thread that links this wide range of activities is that they all use LAMPF beams. We believe that the breadth of the science and technology pursued at LAMPF in conjunction with other LANL divisions, as well as outside users, is a fundamental strength of the institution.

The programs described in this section are funded by several sources, including Basic Energy Sciences, Fusion Energy, Nuclear Energy, Advanced Energy Concepts, Nuclear Weapons Technology, Health and Environmental Research, and LANL's discretionary institutional research program. Very litule, if any, funding originates with the DOE Division of Nuclear Physics.

\section{Mass Measurements and Exotic Decay Studies with TOFI}

\section{Introduction}

The Time-of-Flight Isochronous (TOFI) spectrometer is a unique instrument for studying the properties of very neutron-rich low to medium $\mathbf{Z}$ nuclei. Measurements with TOFI have, for the first time, determined the masses of over 60 neutron-rich nuclei for the elements $\mathrm{Li}$ up to Fe. These measurements have led to: (1) a better understanding of the nuclear shell model, (2) insights into the rapid changes in nuclear shapes occurring in low- $Z$, neutron-rich nuclei, and (3) the nature of nuclear pairing. The mass measurement program is being extended to include elements above $\mathrm{Fe}$ and a collaborative experiment to test the idea of a circular time-of-flight mass spectrometer with ten times the resolution of TOFI. Such an instrument could open up the entire fission product region and yield well over 100 new mass measurements. In addition, a program to study the decay properties of $\beta$ delayed neutron emitters is being developed and a first set of results has been obtained.

The TOFI program involves a collaboration of scientists from Los Alamos National Laboratory, Utah State University, University of Giessen (Germany), Pacific Northwest Laboratories, and the University of New Mexico. Four students have obtained their doctorates in this program.

\section{TOFI Mass Measurement Program}

The ability to measure the masses of far from stability neutron-rich nuclei relies on a unique time-offlight isochronous recoil spectrometer. TOFI can systematically measure the masses of fast recoiling nuclei produced through interactions of the $800-\mathrm{MeV}, 1-\mathrm{mA}$ proton beam with a thin $\left(1-\mathrm{mg} / \mathrm{cm}^{2}\right) \mathrm{Th}$ target. TOFI's unique isochronous design leads to ions with the same mass-to-charge ratio taking the same amount of time to pass through the spectrometer, regardless of their velocity. Thus, a precise measurement of an ion's time-of-flight is a direct measurement of its mass-to-charge ratio. The charge state and atomic number of each recoil is determined from additional measurements of the ion's velocity, stopping power, and total kinetic energy.

One of the first experiments performed using TOFI was the mass measurement of low- $Z$, neutron-rich nuclei including the neutron halo nuclei ${ }^{11} \mathrm{Li},{ }^{14} \mathrm{Be}$, and ${ }^{17} \mathrm{~B}$. These particular measurements confirmed the very low two-neutron binding energy, which is thought to be responsible, in part, for an extension of the neutron mass distribution to large radii. This same experiment also measured the very neutron-rich oxygen isotopes which led 


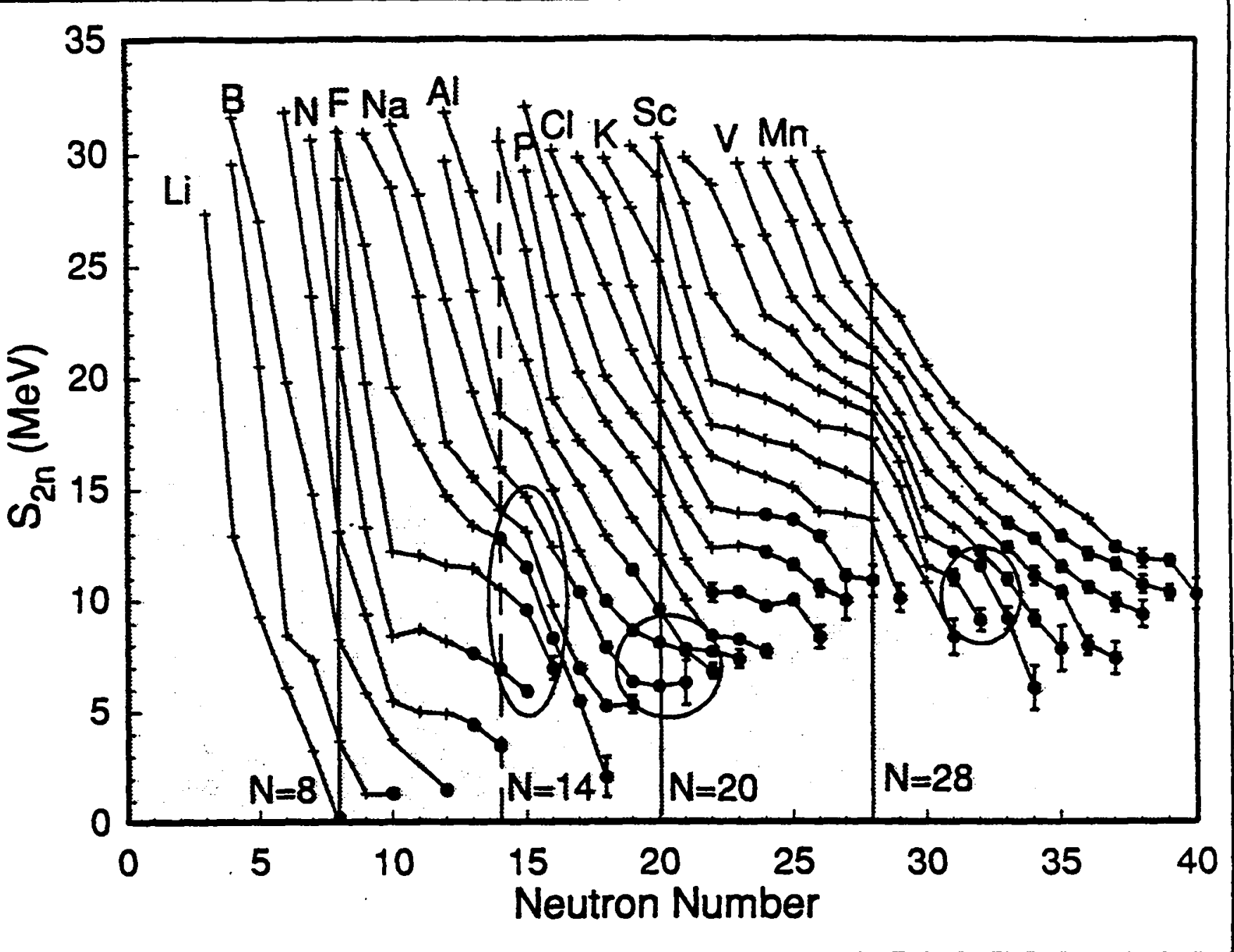

Fig. IV-1. Two neutron-separation energies as a function of neutron number where: - are the TOFI measurements; + are the masses taken from a recent compilation by Audi and Wapstra. The elliptical regions highlight, from left to right, the $N=$ 14,15 anomaly, the ${ }^{31} \mathrm{Na}$ enhanced binding region, and the ${ }^{53} \mathrm{SC}$ enhanced binding region (see text).

to an understanding of the unexpected change in the twoneutron separation energies $\left(S_{2 n}\right)$ systematics which occurs at $N=15$ (as opposed to $N=14$ where the neutron subshell occurs) for all elements from oxygen to silicon (see left ellipse in Fig. IV-1). The good agreement between these mass measurements for ${ }^{23,24} \mathrm{O}$ and a set of simplified approximations made to the Unified SD (USD) shell model demonstrated that the key features can be understood in terms of the interplay of the $d_{5 / 2^{-}}-d_{5 / 2}$ and $\mathrm{d}_{5 / 2}-\mathrm{s}_{1 / 2}$ two-body matrix elements and the $\mathrm{d}_{5 / 2}, \mathrm{~s}_{1 / 2}$ single particle energy gap.

Measurements of the isotopes of $\mathrm{Na}, \mathrm{Mg}$, and $\mathrm{Al}$ helped resolve a ten year old mystery about the nature of the enhanced binding seen originally in the most neutronrich isotopes of $\mathrm{Na}$. The measurements confirmed that this enhanced binding actually exists, though it is smaller than originally reported, and that it is very localized to the $\mathrm{N}=20,21$ isotones of $\mathrm{Na}$ and $\mathrm{Mg}$ as can be seen from the up turn in the $S_{2 n}$ systematics shown in the center ellipse of Fig. IV-1. Theorists have been able to show from these results that this island of enhanced binding is caused by the crossing of high-spin $\left(f_{7 / 2}\right)$ and low-spin $\left(d_{3 / 2}\right)$ neutron levels which removes the magicity of $N=20$. Recently, new measurements by TOFI of the neutron-rich $\mathrm{Ca}_{\mathrm{a}}$ and $\mathrm{Sc}$ isotopes have shown a smaller, but similar region of enhanced binding centered around ${ }^{53} \mathrm{Sc}$ (see right ellipse of Fig. IV-1).

Pairing in nuclei is one of the poorest understood contributions to the overall binding energy of a nucleus. Recent theoretical studies have suggested the possibility of an isospin dependence in the pairing term. Mass measurements of the neutron-rich isotopes of Sc through 
$\mathrm{Fe}$, which cover a large isospin range for a few $\mathrm{Z}$ isochains, indicate the presence of a small isospin dependence in neutron pairing and none in proton pairing. This contradiction could arise because the theoretical studies considered data from the entire chart of the nuclides, where the average isospin is strongly correlated with atomic number. Thus the theoretical pairing energy could be reflecting this $Z$ dependence rather than an isospin dependence.

\section{TOFI Decay Measurements}

TOFI can be used to tag each ion according to its $A$, $Z, Q$, and time of arrival to measure the decay properties of $\beta$-delayed neutron emitters. A moderated ${ }^{3} \mathrm{He}$ detector that surrounds the stopping region is then used to record the time at which a delayed neutron arrives. Correlating the ion arrival and neutron observation times permits a reconstruction of the decay signature for a particular ion. Experimental highlights using this technique include the first measurement of the half-lives for ${ }^{25} \mathrm{~F}$ and ${ }^{28} \mathrm{Ne}$, which agree well with the predictions of the USD shell model, and the first observation of neutron emission from ${ }^{14} \mathrm{~B}$ and ${ }^{18} \mathrm{~N}$. The existence of delayed $\mathrm{n}$-emission from ${ }^{14} \mathrm{~B}$ is particularly interesting in that it could be used to study first-forbidden $\beta$-decay.

\section{Future}

An upcoming experiment using TOFI is a measurement of the masses of several nuclei near the doubly-magic nucleus, ${ }^{78} \mathrm{Ni}$. Masses in this general vicinity will also be of importance to astrophysical rprocess calculations. A program to measure $\beta$-delayed neutron, multi-neutron, and charged particle emission (i.e., $\alpha, t, d)$ with special emphasis on neutron halo nuclei is being developed. This program will use the measured emission probabilities of the various decay channels to reflect back on the underlying nuclear structure of these nuclei.

As an extension of the TOFI mass measurement program, the concept of a circular mass spectrometer is being explored. Such a device would have ten times the resolving power of TOFI such that exotic isobars could be resolved directly. This high resolving power would make possible a whole new set of mass measurements covering the entire $800-\mathrm{MeV}$ proton-induced fission product region as well as provide measurements of even more exotic neutron-rich nuclei in the low Z-region. Inese measurements would address a variety of topics including: (1) the observation of enhanced binding in presently inaccessible regions such as the deformed regions near
${ }^{104} \mathrm{Zr}$ and ${ }^{168} \mathrm{Gd}$, (2) pairing-energy' systematics, (3) astrophysical processes, especially with measurements near waiting-point nuclei (i.e., ${ }^{80} \mathrm{Zn},{ }^{130} \mathrm{Cd}$ ), and (4) a validation of a variety of models such as the $N_{p} N_{n}$ scheme over a wide isospin range. To test the principle of a circular mass spectrometer we are participating in a collaboration that will use the heavy-ion storage ring (ESR) at GSI for a few selected mass measurements. The success of this experiment would verify this approach and make possible the development of a modest-cost circular mass spectrometer at LAMPF with which to measure well over 100 new masses from $Z=5$ to 65 .

The TOFI mass measurement program has measured over 60 masses for neutron-rich isotopes of $\mathrm{Li}$ up to $\mathrm{Fe}$ and led to: (1) a better understanding of the nuclear shell model, (2) insights into the rapid changes in nuclear shapes occurring in low-Z, neutron-rich nuclei, (3) and the nature of nuclear pairing.

The TOFI mass measurement program has measured over 60 masses of neutron-rich isotopes ranging from ${ }^{11} \mathrm{Li}$ to ${ }^{66} \mathrm{Fe}$.

\section{Neutron Scattering at LANSCE}

The need for neutron scattering facilities has been articulated by numerous high-level committees over the past decade. The case is built upon the fact that neutrons provide unique structural information about solids and liquids that are important in fields as diverse as condensedmatter physics, chemistry, materials science, engineering, and structural biology.

The importance of neutrons as a structural probe is rooted in their physical interactions with matter. These interactions are far weaker than the electromagnetic or electrostatic interactions experienced by $x$-rays or electrons, allowing neutrons to penetrate into bulk specimens and through barriers required for sample containment. Furthermore, weak scattering is far easier to interpret unambiguously than strong scattering. Neutrons interact through their magnetic dipole moment both with nuclei and with unpaired electrons, making them suitable probes for both the atomic and magnetic structure of matter. Wavelengths of thermal neutrons are comparable with interatomic spacings in solids and liquids, and their energies are similar to the energies of thermal agitations in matter. These properties allow neutrons to be used to make sensitive measurements of interatomic distances and the energies of atomic motions.

Several of the U.S. neutron scattering centers are now open to users from academia, federal laboratories and industry. In each case, the work performed is peerreviewed and is chosen to support the mission of the 
government department that funds the neutron center. Typically, the demand for beam time exceeds that available by slightly more than a factor of two, but experience in both the U.S. and Europe would indicate that this probably underestimates the true demand.

The Manuel Lujan Jr. Neutron Scattering Center (LANSCE) at Los Alamos National Laboratory is a spallation source that uses protons from the Los Alamos Meson Physics Facility (LAMPF) to generate pulses of neutrons for scattering research. The facility boasts a higher peak flux of neutrons than any other spallation neutron source, often allowing experiments to be performed more quickly, more accurately, and with smaller samples $(-25 \mathrm{mg})$ than at other facilities of this type. LANSCE has several modern neutron spectrometers and a potential for expansion that is unrivaled by any other neutron source in the world.

LANSCE has capabilities that are unique in the U.S. or worldwide: (1) A powder diffractometer with a resolution better than any other similar spectrometer in the U.S. is capable of measuring residual strains in technologically important low-stress materials, such as ceramics and compacted powders. (2) A recently-installed spectrometer, designed for the measurement of inelastic scattering, allows scientists to probe aspects of the dynamics of amorphous materials that have been hitherto inaccessible. (3) The second phase of this spectrometer, which is almost complete, will allow measurements on high-temperature superconductors that scientists from AT\&T Bell Laboratories have had to perform in Europe, because no suitable equipment exists in the U.S. (4) The reflectometer at LANSCE is capable of exploring the structure of liquid surfaces and surfactant layers, as well as the texture of protective coatings and films used for magnetic recording. (5) LANSCE is the only facility in the world to have recorded a transmission diffraction pattern during a single beam pulse, opening up the possibility for studies of the structures of materials subjected to rapidly changing conditions such as shock waves. (6) No other small-angle scattering facility in the world has the dynamic range offered by the LANSCE facility-a great advantage for studies of materials in which differing length scales are important. (7) This year, diffraction experiments will be carried out at LANSCE with samples subjected to pressures of $10^{5}$ times atmospheric pressure - more than three times higher than any previous measurement of this type in the U.S.

More than 200 scientists from academia, federal laboratories and industry requested beam time at LANSCE last year. This number would surely have been higher had beam time not been limited by the insufficiency of the LAMPF operations budget. Almost all of the groups that carried out experiments included one or more graduate students or postdoctoral fellows, with about $40 \%$ of the visiting experimenters falling into one of these two categories.

Many of the projects undertaken at LANSCE have important technological applications. For example, studies of residual stresses in engineering materials have been pursued in collaboration with industrial scientists from the automotive and aerospace industries. Several scientists from petrochemical companies have sought to purchase neutron beam time.

Over the past two decades, the U.S. has fallen further and further behind Europe in the area of neutron scattering. U.S. scientists have repeatedly complained that they do not have access to the sophisticated instruments that have been developed in Europe or to facilities such as those provided by the high-flux reactor at the Institut LaueLangevin in Grenoble, France, or the spallation source at Rutherford-Appleton Laboratory in the U.K. Neutron scattering experiments that contribute to the understanding of such important materials as battery electrolytes, hightemperature superconductors, polymers, catalysts, and biological materials must be carried out in Europe, because there are no facilities in the U.S. that can make the appropriate measurements. This situation severely handicaps U.S. scientists. Following the recommendations of the 1984 panel on Major Materials Facilities, efforts have been made to redress this situation by constructing a cold-neutron facility at the National Institute of Standards and Technology (NIST) and by augmenting the instrumentation available at LANSCE. Both of these programs are slowly reversing the sorry trend of the past twenty years.

Neutron scattering research covers a broad spectrum of topics. Below, we give a few examples to demonstrate the impact of neutron scattering in several important areas and highlight collaborative research that is being carried out with university, Laboratory and industrial scientists.

\section{Metal Matrix Composites}

Metal matrix composites (MMCs) are increasingly replacing conventional materials in structural applications that require high strength-to-weight or stiffness-to-weight ratios. The benefits of improved efficiency die to lighter components are encouraging both the aerospace and automotive industries to research applications for MMCs. Residual stresses between the constituent phases strongly affect the strength and fracture resistance of MMC materials. Conventional techniques of measuring residual stress are hard to apply to composites and are limited because of their inability to distinguish between the 
matrix and reinforcement. Neutron diffraction, which can only be performed at a high-flux neutron source, offers a unique non-destructive alternative for examining the stress state of crystalline composites.

At LANSCE, we use neutron diffraction to examine a variety of MMC materials and to make direct comparisons with computational models. Our experiments confirm the ability of models to predict qualitatively, and in several cases quantitatively, the internal stresses in composite materials. However, there are some intriguing differences between the experimental results and the predictions. In particular, the behavior of the reinforcement is harder to model than the matrix, and the form of the reinforcement, either fibers or particulates, affects the validity of the models. Our experimental results show the limitations of the computer simulations and indicate where enhancements are needed.

Several experiments have been carried out to study residual stresses in more conventional materials, such as welded plates and connecting rods from an automobile engine. Studies of this sort are important examples of the way in which facilities at National Laboratories can contribute to the enhancement of U.S. industrial competitiveness.

\section{Molecular Biology}

Studies of the structures of biological molecules are a natural complement to the Laboratory's Human Genome program. The latter emphasizes the sequence of bases that make up a particular chromosome, whereas structural biology, as studied by neutron scattering, concentrates on the structure and conformation of larger macromolecular units. If the structure of a biological molecule can be determined under appropriate conditions (c.8., in solution), information about its function can often be inferred. Small-angle scattering can contribute significantly to such studies because samples may be prepared in conditions that closely approximate the normal physiological environment - an advantage not shared by techniques like electron microscopy and $x$-ray crystallography. Similar ranges of molecular size can be probed by either $x$-ray or neutron small angle scattering; however, neutron scattering offers the advantage that the scattering contrast between different parts of a molecuiar assembly, or between molecules and solvent, may be controlled by selectively deuterating different components.

The Low-Q Diffractometer at LANSCE has been used to study a number of biological systems, such as calmodulin, phosphorylase kinase, and antibodies. The calmodulin molecule is of great importance as the principal mediator of the regulation of biological processes that are controlled by $\mathrm{Ca}^{2+}$ ions. Examples include muscle contraction and the metabolism of glycogen. In an application of unique capabilities at LANSCE, resonant small-angle neutron scattering was measured from ${ }^{240} \mathrm{Pu}^{3+}$ ions bound to the $\mathrm{Ca}^{2+}$ binding sites of calmodulin. The distance between binding sites within each of the two "globules" that make up the calmodulin molecule was determined with considerable accuracy, but no reasonable distribution of distance between the globules has yet been found to fit the data. Nevertheless, it is clear that, under physiological conditions, calmodulin does not adopt the rigid extended structure determined by $x$-ray crystallography.

\section{Polymers}

Synthetic macromolecules at solid or fluid interfaces have an enormous spectrum of applications to a wide variety of technologies. They provide a mechanism to achieve colloid stabilization in water treatment, ceramic processing, inks, fuels, and other suspensions and emulsions; they are used for mechanical protection of solids against friction and wear, they are used as model biomembranes; and they are being developed for optoelectronic devices.

Although there is a substantial body of theoretical literature on the morphologies of adsorbed polymers, grafted chains, block copolymers etc., for the most part, experimental evidence has been inferential. In an attempt to correct this situation, experiments are underway at LANSCE to probe the structure of macromolecular layers by neutron reflection. This technique permits the determination of the average density profile perpendicular to flat layers, as well as providing information about the texture or roughness of the layers. By combining neutron reflection sudies with surface force measurements, we aim to further the understanding of the interactions within complex fluid layers. As a first step, neutron reflection measurements have been made on polymer molecules that were anchored to a solid/liquid interface by a lipid bilayer. By changing the density of the lipid layers, the grafting density of the polymer "brush" extending into the liquid can be controlled. When fully analyzed, the data are expected to yield information about the density of the polymer "brush" that can-be compared with recent theories. Experiments have also been conducted on a related system of diblock copolymers. Under suitable solvent conditions, block copolymers can adsorb with one compact, tightly bound block and one extended, swollen block (a brush) stretching away from the surface. The reflectivity profiles of this system are well described by parabolic as well as error function density profiles normal 
to the interface, but the data cannot be fitted to exponential or power-law decay profiles. These experiments represent the first confirmation of this theoretically predicted behavior. The molecular weight dependence of the layer thickness and adsorbance obtained from the data obey scaling laws in accord with the theory of polymer "brushes."

\section{Structure of Rare-Earth Oxides}

Neutron powder diffraction was used to solve the structures of five members of the homologous series $\mathrm{M}_{\mathrm{n}} \mathrm{O}_{2 \mathrm{n}-2}$ for $\mathrm{M}=\mathrm{Pr}$ and $\mathrm{Tb}$. All of these structures are derived from the parent $\mathrm{MO}_{2}$ fluorite by creation of oxygen vacancies and subsequent lattice distortion. All have at least one common cell edge that is one-half of the [211] vector in fluorite. Apart from $\mathrm{Tb}_{7} \mathrm{O}_{12}$, these materials have remarkably low symmetry and very large unit cells; most are either monoclinic or triclinic. One of the largest of the structures solved is $\operatorname{Pr}_{10} \mathrm{O}_{18}$ whose monoclinic unit cell contains 112 atoms and is described by 84 atomic coordinates. The basic trend observed in a comparison of these structures is that in the higher oxides, where the mean lattice volume for each vacancy is -200 cubic angstroms or larger, the vacancies are no longer paired on opposite sides of a metal atom as found in the rhombohedral $\mathrm{M}_{7} \mathrm{O}_{12}$ structure. This is consistent with the fact that the dominant bonding force in rare-earth oxide systems is mainly ionic in nature. Thus, the formation of any intermediate phase in the homologous series is the result of minimizing the interactions between defect centers and does not involve any covalency effects which might preserve a large defect cluster in a dilute array of vacancies.

These oxides are typical of a large group of structures based on ordering of extended defects formed by oxygen vacancies in a $\mathrm{MO}_{2}$ fluorite lattice. Similar structures are found for the oxides of $\mathrm{Pu}, \mathrm{Am}, \mathrm{Cm}, \mathrm{Bk}$, and $\mathrm{Cf}$, as well as the ternary oxides of $\mathrm{Zr}, \mathrm{Hf}$, and $\mathrm{Th}$ with $\mathrm{Ca}$ or the rare earths. The latter materials have potential use as nuclear fuels, fast oxygen conductors (solid electrolytes), and oxygen specific sensors.

\section{Nuclear Physics Studies with Neutrons at LANSCE and WNR}

\section{Background}

Together, LANSCE (Los Alamos Neutron Scattering Center) and WNR (Weapons Neutron Research Facility) provide very high-intensity "white" neutron beams with energies ranging from thermal to $800 \mathrm{MeV}$. During the
1990 LAMPF run, 107 scientists fielded 19 nuclear physics experiments at these facilities; 58 of these scientists were from outside Los Alamos and came from 11 U.S. universities, 5 foreign universities, 3 U.S. national laboratories, 5 foreign laboratories, and 3 U.S. companies. Numerous graduate students use these facilities, and four of them have received Ph.D. degrees over the last two years. Researchers at these facilities conceived the unique Accelerator Transmutation of Waste (ATW) concept, which is now being pursued at Los Alamos. In the following, we highlight some of the ongoing and future proposed research at these facilities.

\section{Ongoing Research}

\section{Parity and Time-Reversal Tests in Compound Nuclei}

A collaboration between LANL and outside users is studying parity and time-reversal invariance violations using a polarized epithermal neutron beam at LANSCE. A summary of this research appears elsewhere in this document and, so, is not repeated here.

\section{Nuclear Astrophysics}

Reactions involving radioactive nuclei are currently of high interest in experimental nuclear astrophysics; however, to date, very few measurements have been made of neutron-induced cross sections on radioactive samples, mainly because of the potentially large backgrounds associated with the target being studied and the difficulty of obtaining suitable samples. Recently, measurements have been made on samples with half lives as short as a few months, with as little as $75 \mathrm{ng}$ of the radioisotope. Published measurements include ${ }^{7} \mathrm{Be}(\mathrm{n}, \mathrm{p})^{7} \mathrm{~L} \mathrm{i}$, ${ }^{14} \mathrm{~N}(\mathrm{n}, \mathrm{p}){ }^{14} \mathrm{C},{ }^{17} \mathrm{O}(\mathrm{n}, \mathrm{a}){ }^{14} \mathrm{C},{ }^{2} \mathrm{Na}(\mathrm{n}, \mathrm{p})^{22} \mathrm{Ne},{ }^{35} \mathrm{Cl}(\mathrm{n}, \mathrm{p})^{35} \mathrm{~S}$, and ${ }^{36} \mathrm{Cl}(\mathrm{n}, \mathrm{p})^{36} \mathrm{~S}$.

The measurements have applications in both standard and nonstandard big-bang, explosive-stellar, and s-process nucleosynthesis. For example, there has recently been a great deal of interest in the possibility of synthesizing "metals" $(A \geq 12)$ in nonstandard models of the big bang. Such metals have been seen in even the oldest stars observable an' cannot be explained by standard big-bang models, which do not synthesize elements heavier than ${ }^{7} \mathrm{Li}$. It has been speculated that, as a possible explanation of these metal abundances, the nonstandard big-bang hypothesis may be distinguishable from one involving early supermassive (so-called Population II) stars by the different isotopic and/or elemental signatures created in the two environments. However, some of the imporant cross 
sections for these calculations were unmeasured, so the conclusions were rather speculative. Our recent data on the ${ }^{14} \mathrm{C}(\mathrm{n}, \gamma){ }^{15} \mathrm{C}$ and ${ }^{17} \mathrm{O}(\mathrm{n}, \alpha){ }^{14} \mathrm{C}$ reactions show that ${ }^{17} \mathrm{O}$ and ${ }^{14} \mathrm{C}$ act as a bottleneck to heavy-element nucleosynthesis in nonstandard big-bang models. Hence, it is unlikely that these models will be able to explain the metal abundances seen in the oldest stars.

The combination of large neutron flux available at LANSCE and local resources, such as the isotopeproduction facility at LAMPF, isotope separators, and radiochemistry laboratories, place us in a unique position to make many other measurements of astrophysical interest on short-lived radioactive samples.

\section{Neutron-Proton Bremsstrahlung}

Neutron-Proton Bremsstrahlung (NPB), the emission of gamma rays during inelastic neutron-proton collisions, is an off-shell process, where the contributions of mesonexchange currents have been calculated to be large. Only sparse data presently exist to verify the calculations of meson-exchange currents in the nuclear interaction. NPB experiments are difficult because of the low intensity of neutron beams and the problems associated with detecting neutral particles in a high-background environment. Only four direct measurements of NPB exist, all with poor neutron energy resolution, and all have averaged over large areas of phase space. Studies of NPB at WNR will provide the first differential measurement of the NPB cross section over a wide range of neutron energies.

The first stage of these studies was initiated during the 1991 LAMPF run with a measurement of inclusive gamma-ray production at $90^{\circ}$ with respect to the beam. Two gamma-ray detector telescopes were located on either side of a liquid-hydrogen target. Preliminary analysis of these early data shows reasonable agreement with theory. The second stage is planned for the 1992 LAMPF run and will involve detection of the scattered proton at small forward angles, in coincidence with the gamma ray. This is an extremely interesting regime because it accesses far off-shell contributions to the nuclear potential. The third stage involves the addition of neutron detectors, so that all outgoing particles will be detected.

This experiment will ultimately provide a complete measurement of this fundamental nucleon-nucleon process from $50-\mathrm{MeV}$ to $400-\mathrm{MeV}$ incident neutron energy. These data will serve as crucial input to calculations aimed at understanding the origin of high-energy gamma rays observed in heavy-ion collisions.
Gamow-Teller and Giant-Resonance

Excitations in $(\mathbf{n}, \mathbf{p})$ Reactions

The continuous energy spectrum of the WNR "white" neutron source has opened new possibilities for studying the energy dependence of isovector (charge-changing) excitations in nuclei. The $(n, p)$ reaction at energies from $50 \mathrm{MeV}$ to $250 \mathrm{MeV}$ has been used to study several problems that have been at the forefront of intermediateenergy nuclear physics research in recent years - viz., Gamow-Teller excitations, giant resonances and the highexcitation cortinuum. These experiments employ a "wall" of CsI detectors to measure the proton energy. The neutron energy maps out the region of rapid change in the ratio of the effective interactions for isovector spin to nonspin and, therefore, provides a tool for studying spin versus nonspin excitations.

The first stage of the experimental program concentrated on Gamow-Teller strength in light nuclei. These experiments provided a "calibration" of cross section versus measured beta-decay strengths. Of particular interest was the first measurement of the ratio of the cross section at zero momentum transfer to the Gamow-Teller beta-decay strength for the $(n, p)$ reaction in the fp-shell, using the ${ }^{64} \mathrm{Ni}(\mathrm{n}, \mathrm{p})$ reaction.

The second stage involved studying giant resonances and Gamow-Teller strength in heavier self-conjugate nuclei, ${ }^{32} \mathrm{~S}$ and ${ }^{40} \mathrm{Ca}$. Figure IV-2 shows the ${ }^{40} \mathrm{Ca}(\mathrm{n}, \mathrm{p})$ response surface at $7^{\circ}$ in the lab. The giant dipole resonance (GDR) is obvious as a single bump at 12.5$\mathrm{MeV}$ excitation, at a neutron energy of $60 \mathrm{MeV}$. At the highest beam energy, it has been replaced by a broader structure identified as the giant spin-dipole resonance (GSDR). This figure demonstrates the richness of data attainable at WNR - numerous other peaks, presumably corresponding to other giant resonances, are evident in this three dimensional plot. The dual dependence on excitation energy and neutron kinetic energy causes them to stand out above background processes.

\section{Proposed Future Research}

\section{Isotropic Polarized Thermal Neutron Source}

An exciting new idea, still in the conceptual-design stage, is the production of an intense flux of polarized thermal neutrons. The idea is to polarize neutrons using laser-polarized ${ }^{3} \mathrm{He}$ gas, placed inside a large graphite block, which thermalizes neutrons. A proton beam would impinge on a heavy metal target that is surrounded by the large graphite block. The ${ }^{3} \mathrm{He}$ will capture neutrons with a given polarization, while being transparent to neutrons 


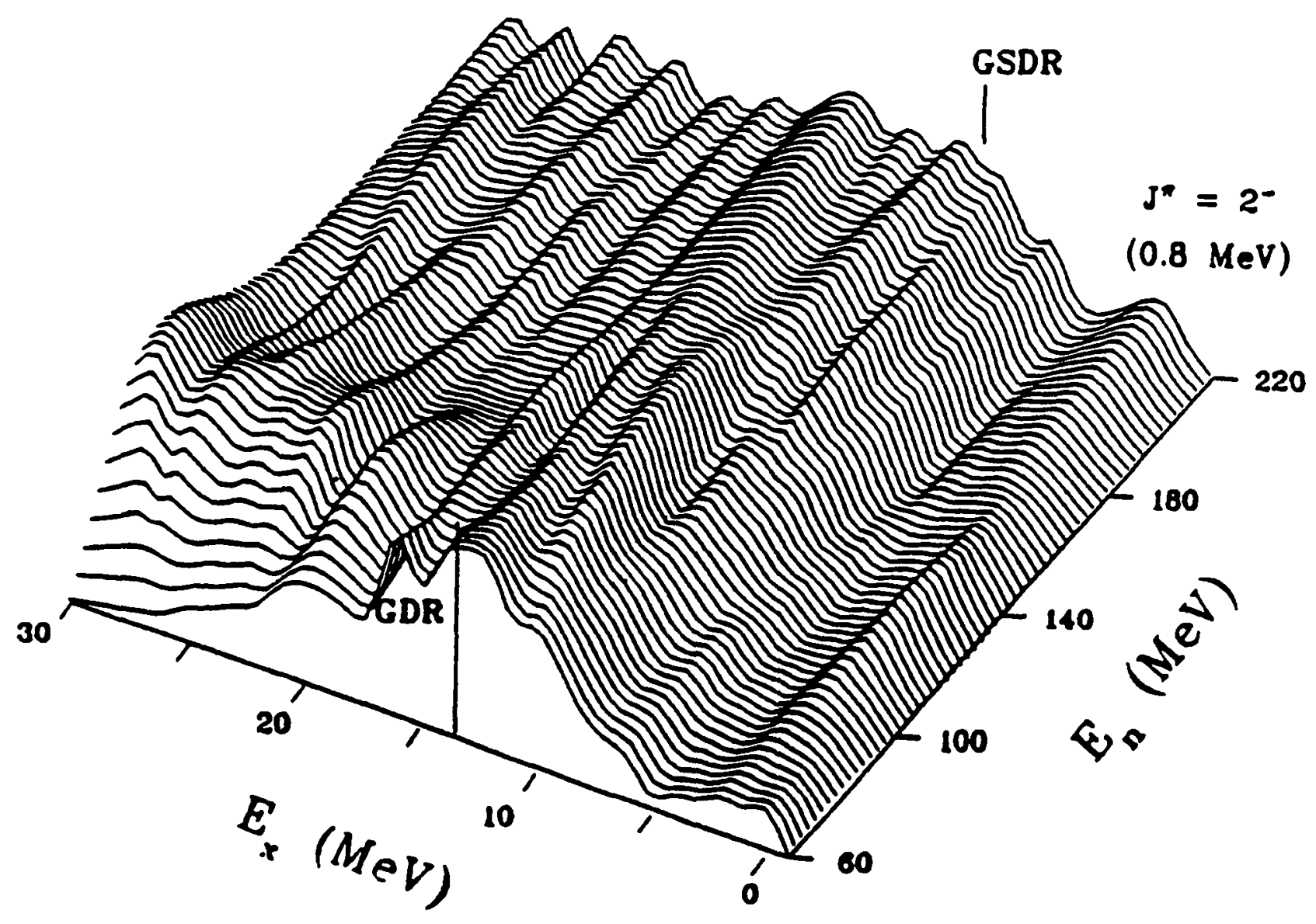

Fig. $N-2$. The ${ }^{40} \mathrm{Ca}(n, p)$ response surface at $7^{\circ} \mathrm{lab}$. The neutron beam energy is shown along the right axis and excitation energy along the left axis. The giant dipole (GDR) and giant spin dipole (GSDR) are indicated.

of the opposite polarization. One can expect to a achieve polarized neutron flux of $10^{14} \mathrm{n} \mathrm{cm}^{-2} \mathrm{~s}^{-1}$ using the LAMPF beam. This flux, which is 5 orders of magnitude larger than the best available polarized neutron beams, would enable the investigation of effects such as the isovector component of parity violation and the spin dependence of gravity. It might also make possible some very sensitive experiments that search for time-reversal invariance violation, which is of great importance for testing the Standard Model.

\section{Dibaryon Resonance}

The question of the existence of dibaryon resonances is an outstanding problem in nuclear physics. There are several six-quark, $B=2$ resonance candidates, published in recent particle-property compilations; however, they are not firmly established. A recent work by Hoshizaki and Watanabe attempts to elucidate the situation in $n-p$ scattering above $400-\mathrm{MeV}$ lab energy. Their phase shift solution shows a narrow isospin- $0, \pi^{\pi}=1^{-}$resonance at an invariant mass of $2168 \mathrm{MeV} / \mathrm{c}^{2}$ (lab kinetic energy of $625 \mathrm{MeV}$ ). This resonance could be confirmed by a highstatistics, high-resolution total cross-section measurement at WNR. A better measurement would result from developing a polarized beam at WNR, which would significantly enhance the signiature of the resonance.

\section{Quantum Chaos}

In recent years, the subject of quantum chaos has become of great fundamental interest. Attempts have been made to determine whether classically chaotic systems have quantum analogs, and, if so, how many chaotic 
quantal systems can be recognized. The Bohigas conjecture states that the eigenvalues of systems that are chaotic in the classical limit have statistical properties characteristic of the eigenvalues of real symmetric matrices with randomly distributed matrix elements. This theory implies specific statistical properties of eigenvalue spacings, which are evident in level sequences of slowneutron resonances. The problem is that the theory should be applied to levels with a single good set of quantum numbers. In practice, neutron resonances, which are generally s-wave, contain a significant number of $p$ wave states. In previous studies, no physical identification of the p-wave resonances was made, and the methods used were not adequate to locate weak resonances of any kind.

The high neutron intensities available at LANSCE are such that greatly improved resonance studies could be made using capture gamma-ray cross-section measurements. These would give much greater sensitivity to weak resonances, and allow positive identification of the spin and parity quantum numbers. To answer more detailed questions about chaos in quantum systems, measurements of transition strengths and level spacings are needed. Additional information may also be obtained from fission (in the heaviest nuclei), as well as from a and p decay channels, where detectable. Thus, LANSCE could open up new vistas in slow-neutron spectroscopy, with the main focus on the subject of quantum chaos. These studies also have a significant bearing on parity and timereversal invariance violation, nuclear structure, and nuclear reaction mechanisms.

\section{Muon Spin Research}

A modest but productive muon spin rotation/ relaxation ( $\mu S R$ )-materials science program has been a part of the LAMPF experimental effon for many years. Initial research focused on the behavior of muons in $\mathrm{Cu}$ and Al; subsequent work addressed magnetic behavior of spin glasses and heavy fermions. Recent investigations into the superconducting and magnetic properties of hightemperature superconductors (HTSs) have demonstrated the unique capabilities of $\mu S R$. For example, $\mathrm{Y}_{1-\mathrm{x}} \mathrm{Pr}_{\mathrm{x}} \mathrm{Ba}_{2} \mathrm{Cu}_{3} \mathrm{O}_{7}$ is superconducting for $\mathrm{x}<0.6$ and magnetic for $x>0.6$, with an overiap region where the two states coexist. The magnetic phase diagram (see Fig. IV-3) of this material was recently established at LAMPF by zero-field $\mu$ SR, which utilized the extreme sensitivity of the $\mu^{+}$to local magnetic fields. Conventional techniques, such as nuclear magnetic resonance and neutron scattering, could not elucidate the magnetic phase diagram. The unique information obtained at LAMPF by $\mu$ SR was very important because it provided insight into the interplay between magnetism and superconductivity in HTS, which is paramount to establishing the mechanism responsible for the formation of ground states in these exotic materials.

In FY91, the $\mu S R$ program took a new and exciting turn: instead of time-differential $\mu S R$ that had been done previously, a time-integral technique, muon level-crossing resonance spectroscopy ( $\mu L C R$ ), was begun. Timedifferential methods limit the $\mu^{+}$stopping rate and thereby suffer a very drastic data rate penalty at LAMPF because of the low duty factor. However, with time-integral methods, all of the muon flux available at the stopped muon channel $\left(\sim 2 \times 10^{7} \mu^{+} / s\right)$ can be effectively used.

Muon level-crossing resonance experiments attempt to find magnetic field values for which the $\mu^{+}$ polarization, initially parallel to the field, transfers resonantly to a neighboring nucleus. The resulting spectra yield important information on the $\mu^{+}$location in the crystalline lattice and the electron spin density, if any, on neighboring nuclei. For example, breakthroughs in understanding the behavior of hydrogen in semiconductors, muonium radical structure, hyperfine coupling in $\mathrm{CuCl}_{2}$. and oxygen positions in $\mathrm{YBa}_{2} \mathrm{Cu}_{3} \mathrm{O}_{7}$ have recently resulted from $\mu \mathrm{ICR}$ studies.

During the summer of 1991 , an international collaboration began pursuing $\mu L C R$ at LAMPF. Beam development studies culminated in measuring the $\mu L C R$ spectrum of $\mathrm{Cu}$; the results are shown in Fig. IV-4. The resonance occurred at 80 Oe with FWHM of 25 Oe. These results, combined with the high muon flux and corresponding high data rate, demonstrate that LAMPF can be developed into the world's leading $\mu \mathrm{LCR}$ facility.

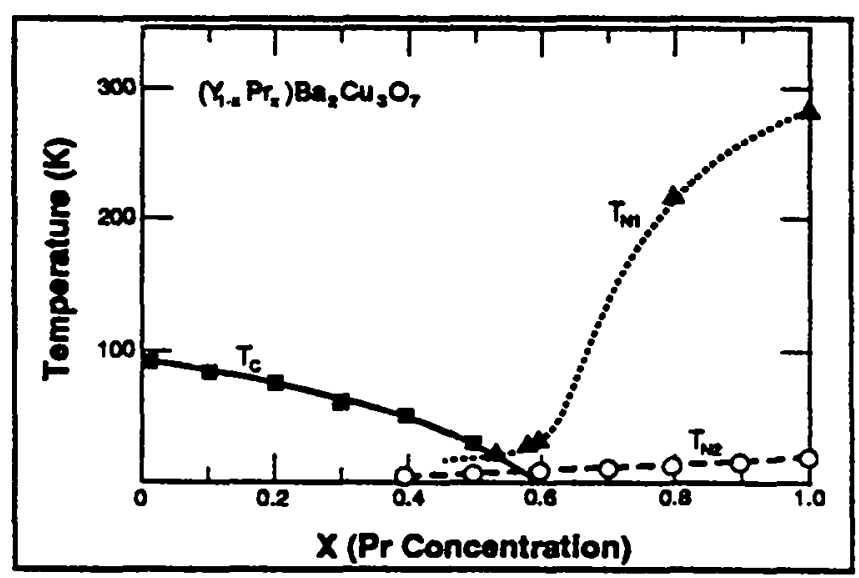

Fig. IV-3. Magnetic phase diagramfor $\mathrm{Y}_{1-x} \mathrm{Pr}_{x} \mathrm{Ba}_{2} \mathrm{Cu}_{3} \mathrm{O}_{7}$. $T_{N 1}$ corresponds to antiferromagnetic ordering of $C u$ moments within the $\mathrm{Cu}-\mathrm{O}$ planes as determined by zerofield $\mu S R$. Spin-glass-like magnetism occurs for $x<$ 0.54 . 


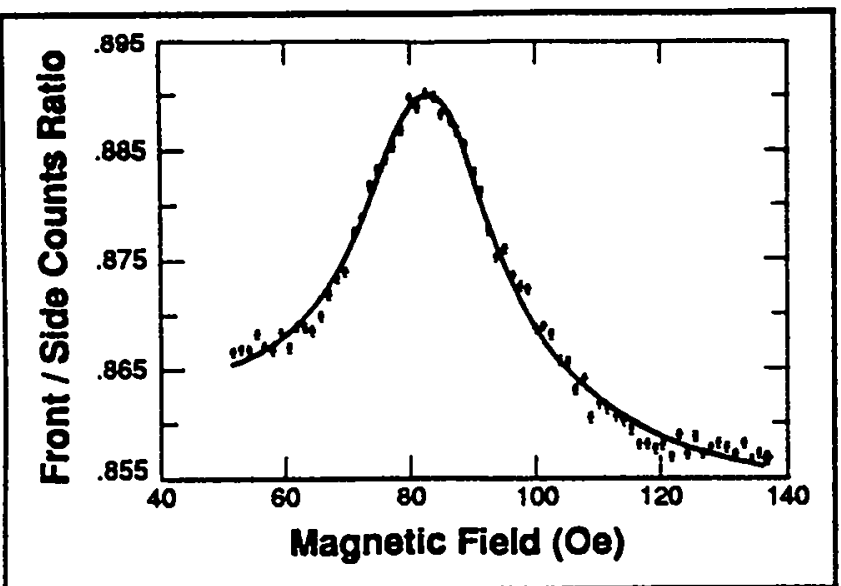

Fig. IV-4. $\mu L C R$ spectrum of single-crystal $C u$. The resonance occurs at 80 Oe with FWHM of $25 \mathrm{Oe}$.

\section{Muon-Catalyzed Fusion}

LAMPF has played a leading role in the revival of interest in muon-catalyzed fusion $(\mu \mathrm{CF})$ that began in the early 1980s. The basic catalysis cycle is sketched in Fig. IV-5. The startling prediction of $100 \mathrm{~d}-t$ fusions per $\mu^{-}$, made in 1977 by Gershtein and Ponomarev, was first verified by the LAMPF experiment E1151. This and subsequent LAMPF work provided important confirmation of the resonant nature of $(\mathrm{d} \psi \mathrm{H})$ formation, established the separate temperature dependences of this formation in $(t \mu)+D_{2}$ and $(\mu)+D T$ collisions (see Fig. IV-6), provided the first evidence for a three-body collision mechanism in (dqu) formation, as well as the first

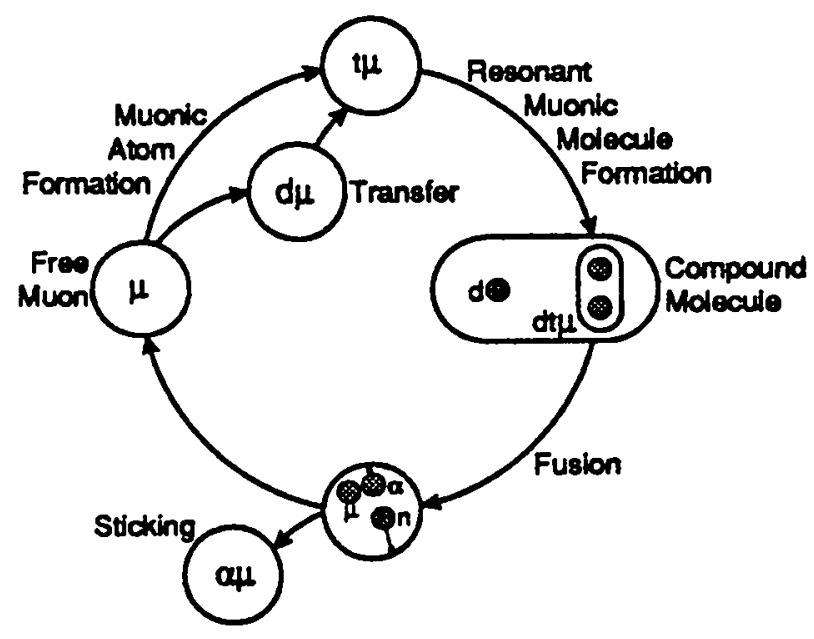

Fig. N-5. The (simplified) d-t muon catalysis cycle.

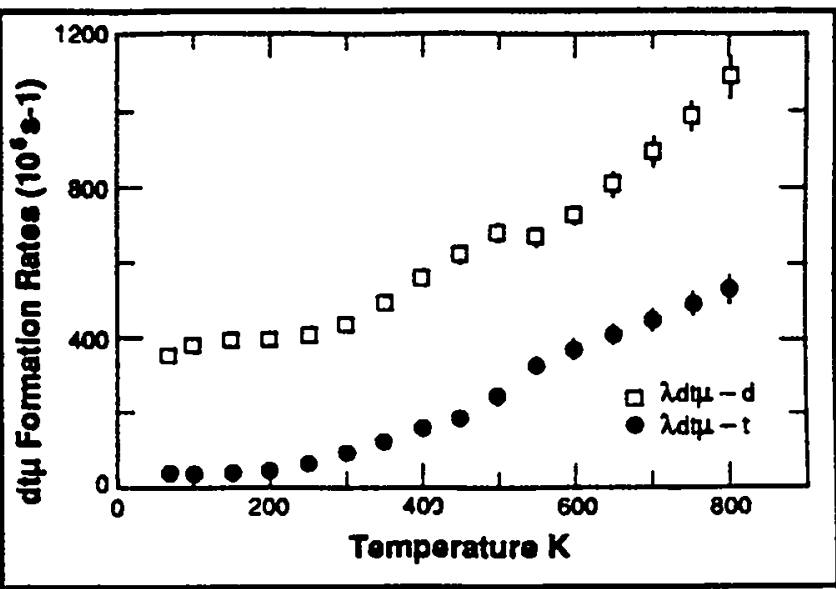

Fig. $N-6$. The temperature dependence of the $t \mu+D_{2}$ (upper points) and $t \mu+D T$ (lower points) molecular formation rates, as deduced from the LAMPF cycling-rate data.

measurement of the "sticking" probability following the $d+t \rightarrow{ }^{4} \mathrm{He}+n$ reaction.

The physics of $\mu \mathrm{CF}$ has turned out to be much richer and more intricate than originally anticipated. For instance, it would be very helpful to know the temperature dependence of the three-body contribution to molecular formation. Experimental $\mu C F$ work at LAMPF was halted in 1990 when the needed LANL tritium facility was shut down because of changing DOE safety and environmental requirements. This was especially unfortunate because there are important areas to be explored that will not be addressed at other muon facilities. One such area is measurements at very high $\mathrm{d}-\mathrm{t}$ densities $\left(2.3 \times\right.$ liquid $\mathrm{H}_{2}$ density), using a very high-pressure ( $7 \mathrm{kbar}$ ) target system, which was about $80 \%$ complete when the tritium facility was closed. A second area is exploring the temperature dependence of the unexpectedly large (du) formation rate on DT molecules, using the existing onekilobar target system and low deuterium fractions.

It is hoped that tritium-facility availability and funding will permit the resumption of this effort in the near future.

\section{Photodetachment of the $\mathrm{H}^{-}$Ion}

Adding a single electron to hydrogen results in the weakly-bound atomic system of $\mathrm{H}^{-}$. Unlike another twoelectron system, atomic helium, $\mathrm{H}^{-}$has no characteristic emission lines to indicate its presence. Like most atomic negative ions, $\mathrm{H}^{-}$is "dark": when either of its electrons is excited, the system tends to shed its excess energy by ejecting one of the electrons rather than by emitting photons of characteristic frequencies. Radiant energy 
emitted from this negative ion is associated with the ejected electron and, therefore, consists solely of photons having a continuous energy distribution. Similarly, the photon-absorption spectrum of $\mathrm{H}^{-}$is continuous. This particular spectrum is of major importance in understanding the opacity of the sun, because most of its thermal photons are absorbed in the continuum transitions of this abundant negative ion.

Beyond its importance for astrophysics, this ion plays a critical role as the simplest quantum-mechanical threebody system; nevertheless, its theoretical description has remained surprisingly elusive. For one thing, the Schroddinger equation cannot be solved exactly. This has not proved to be an insurmountable barrier to gaining an understanding of the ground state of $\mathrm{H}^{-}$, as approximation techniques in quantum mechanics provide a wave function and energy value that are nearly exact. On the other hand, our understanding of the excited states of this ion remains quite limited and approximate at present, despite tremendous enhancements in computational power in recent decades.

A major advance came in the late 1970s when an experimental group at the University of New Mexico and Los Alamos National Laboratory aimed high-intensity pulsed lasers at beams of $800-\mathrm{MeV} \mathrm{H}^{-}$ions produced at LAMPF. An $\mathrm{H}^{-}$ion of this energy has a velocity of $84 \%$ of the speed of light. By firing the laser photons mainly upstream against the onrushing ions, the visible laboratory photons from the lasers are shifted into the "vacuum ultraviolet" frequency region. At certain energies, these photons create two spectacular "doubly excited states" of $\mathrm{H}^{-}$, in which the system briefly contains two excited electrons before shedding an electron. These "resonances" were observed near hydrogen's first excited state $(n=2)$. This work was carried out at LAMPF's High Resolution Atomic Beam Facility (HIRAB).

Recently, families of new resonances have been observed up to the energy level $n=8$. Unlike most familiar resonances, which cause an enhancement of the cross section, these new resonances appear as "windows" in the continuous absorption spectrum; i.e., for certain narrow wavelength regions in the vacuum ultraviolet, the willingness of the ion to shed an electron in exchange for a photon decreases dramatically. Analysis of the resonance energies of these windows showed that they could be described by a remarkably simple relation reminiscent of the Balmer formula. Accordingly, the resonances are grouped into families. The resonance with lowest energy in each family has the two electrons at comparable distances from the proton. On the other hand, all higher resonances within a given family can be viewed more like those of an excited hydrogen atom having a large permanent dipole moment, which in turn binds the more distant outer electron.

\section{Radioisotope Production}

The Medical Radioisotopes Research and Production Programs at Los Alamos make use of the excess beam of $800-\mathrm{MeV}$ protons from the LAMPF accelerator to produce radioisotopes of demonstrated or potential value in nuclear medicine and/or biomedical research, and provide these radioisotopes to the nuclear medicine, biomedical, and other research communities. With the intense beam current of LAMPF, quantities of radioisotopes can be produced that cannot be produced anywhere else in the world. In fact, more than 30 radioisotopes are produced at LAMPF, approximately 15 of which LAMPF is the sole U.S. supplier. Research activities continue to focus on the development of new radioisotopes. Production activities are directed at providing radioisotopes in various stages of development, from strictly research radioisotopes to those used in commercial products. In FY91, personnel from LANL's INC Division made over 120 shipments of $17 \mathrm{Ci}$ of 15 radioisotopes to more than 50 organizations worldwide. These organizations include universities, hospitals, government research institutions, radiopharmaceutical manufacturers, and other commercial entities; the research communities served include nuclear medicine, biomedical research, environmental science, materials science, and physical science. The Medical Radioisotopes Research and Production Programs serve a vital function for the nuclear medicine and other research communities as a national resource for radioisotopes for use in medicine and other applications.

The Isotope Production Facility (IPF) at LAMPF consists of an automated insertion and retrieval system at the LAMPF beam stop that allows target materials, including metals, alloys and saits, to be inserted into the proton beam. There are, currently, nine target stringers that can be inserted and irradiated simultaneously. Targets and irradiation schedules are determined by the requirements of commercial customers and the nuclear medicine research community. Other requirements for radioisotopes are satisfied on a best-effort basis. After irradiation, the targets are highly radioactiv $\left\{>10^{5} \mathrm{Rem}\right)$; therefore, they are remotely removed from the LAMPF beam-stop area, transported to a hot-cell facility, and processed for the radioisotope products.

Several examples of commercial and research radioisotopes produced at LAMPF are listed below. 


\section{Commercial LAMPF-Produced Radioisotopes}

${ }^{68} \mathrm{Ge}$ : This radioisotope is the parent of ${ }^{68} \mathrm{Ga}$, which is a positron-emitter. $A{ }^{68} \mathrm{Ge} /{ }^{68} \mathrm{Ga}$ biomedical generator is available, and radiopharmaceutical development work using this generator as the source of ${ }^{68} \mathrm{Ga}$ is a nuclear medicine research area. However, the major use for this radioisotope is for calibration and absorption correction sources for clinical positron emission tomography (PET) scanners. PET is currently the fastest growing nuclear medicine diagnostic tool, with 100 centers already operating and another 100 projected by 1995. This represents a total capital investment of approximately $\$ 1,000 \mathrm{M}$, and most of this equipment cannot operate without the ${ }^{68} \mathrm{Ge}$ calibration sources. The Department of Energy is currently the only supplier of this important nuclear medicine radioisotope.

${ }^{82} \mathrm{Sr}$ : This radioisotope is the parent of ${ }^{82} \mathrm{Rb}$, which is a positron-emitter. The ${ }^{82} \mathrm{Sr} /{ }^{82} \mathrm{Rb}$ biomedical generator is a commercial product manufactured and marketed by Bristol Myers Squibb Diagnostics. The ${ }^{82} \mathrm{Rb}$ produced from the generator is used clinically in cardiac imaging by PET. Many new PET centers are dependent on this generator for diagnostic procedures, because they do not have on-site cyclotrons to supply radiopharmaceuticals. DOE is the major supplier of this isotope.

$109 \mathrm{Cd}$ : LAMPF is the only North American supplier of this isotope that is employed in portable $x$-ray fluorescence instruments used on the floors of metallurgical shops for analysis of steels.

\section{Research Uses of LAMPF-Produced Radioisotopes}

${ }^{67} \mathrm{Cu}$ : This radioisotope has properties that make it an extremely attractive candidate for radiopharmaceuticals for both diagnostic and therapeutic applications. Research activities include studies of copper metabolism, investigations of autoimmune diseases such as myasthenia gravis, early detection and treatment of lung cancer, as well as diagnosis and treatment of lymphoma and colon cancer. For these applications, high specific- activity material is required. Reactor-produced material does not satisfy this requirement, only accelerator-produced material can be made with adequate specific activity. DOE is the only supplier of high specific-activity ${ }^{67} \mathrm{Cu}$ for these areas of research.

${ }^{72}$ Se: This radioisotope is the parent of ${ }^{72} \mathrm{As}$, a positron-emitter. The development of this ${ }^{72} \mathrm{Se} /{ }^{72} \mathrm{As}$ generator and related arsenic radiopharmaceuticals holds the promise of a wide variety of clinical diagnostic PET procedures without the necessity of an on-site cyclotron. The commercial success of the ${ }^{82} \mathrm{Sr} / 82 \mathrm{Rb}$ generator indicates the potential of generator-produced positron emitters, but the diversity of arsenic chemistry should lead to many more clinical applications than are possible with the Squibb generator. Development of the ${ }^{72} \mathrm{Se} /{ }^{72} \mathrm{As}$ generator, coupled with development of arsenic radiopharmaceuticals, could greatly enhance the clinical utility of PET, because PET centers could be established without the need for an on-site cyclotron. This would allow for the growth of PET into small hospitals and in rural settings, where the use of generator-based PET isotopes such as ${ }^{82} \mathrm{Rb}$ and ${ }^{72} \mathrm{As}$ would be possible. LAMPF is the only supplier of ${ }^{72} \mathrm{Se}$ for this research.

${ }^{88} \mathrm{Y}$ : LAMPF is the only supplier of the ${ }^{88} \mathrm{Zr}$ parent for generating the requisite high specific-activity ${ }^{88} \mathrm{Y}$ used for chemical development of monoclonal antibody labeling with ${ }^{90} \mathrm{Y}$.

26 Al: This radioisotope is used for understanding the role of $\mathrm{Al}$ in Alzheimer's Disease. LAMPF is the only source of this radioisotope.

LAMPF also is the sole or major producer of the following radioisotopes: ${ }^{32} \mathrm{Si}$, which is the only longlived radioisotope of silicon used for environmental research; ${ }^{44} \mathrm{Ti}$, which is the parent for ${ }^{44} \mathrm{Sc}$, a positron emitter that could form the basis of yet another generator for PET; ${ }^{22} \mathrm{Na}$, a positron emitter with a variety of commercial applications; and ${ }^{148} \mathrm{Gd}$, which is used as a low-energy alpha source for physics experiments (on board Galileo).

\section{Studies of the Effects of Radiation on Materials}

A number of programs, worldwide, use the particle radiation sources at LAMPF to study radiation-damage physics and to test and qualify materials for service in accelerator systems and in advanced energy facilities, such as fusion reactors. Both protons and neutrons are used in these studies.

The Superconducting Super Collider (SSC) program uses the proton beam at the High-Resolution Spectrometer (HRS) and the neutron flux at the Los Alamos Spallation Radiation Effects Facility (LASREF) to test the radiation-hardness of semiconductor elements, silicon strip detector elements and carbon-composite structural material for development of the central detector at the SSC. Full integrated-component tests are planned for the future. LASREF is the only facility in the U.S. where these tests can be made.

Critical components requiring tolerance to radiation damage, such as proton-beam entry windows for use at 
LAMPF, PSI and the European Spallation Source, are tested at LASREF. This work will extend to testing of materials for the Accelerator Transmutation of Waste (ATW) and Accelerator Production of Tritium (APT) projects, for which the LAMPF beam energy is ideal.

Measurements of radiation-induced point-defect production, made at LASREF, are used to predict the kinetics of microstructural evolution in materials, and the attendant mechanical and physical property changes.

Researchers supporting materials development for the Office of Fusion Energy (OFE) are using LASREF to study radiation effects in ceramic materials and fiber-optic signal transmission lines. Here, LASREF is most attractive because of ample flux in a very large volume and a demonstrated capability to make in-situ measurements of physical properties.

Approximately 30 publications have resulted from the above work over the past five years.

\section{Astrophysics}

\section{Ultra-High-Energy Gamma-Ray Astronomy}

The CYGNUS experiment at LAMPF detects extensive air showers (EAS) generated by the interaction of Ultra-High-Energy (UHE, $100 \mathrm{TeV}$ - $10 \mathrm{PeV}$ ) cosmic gamma rays with the earth's atmosphere. The purpose is to search for discrete sources of UHE gamma rays (such as neutron stars and black holes), to understand the mechanism of the cosmic accelerators within these sources, and to study the interactions of the gamma rays with the earth's atmosphere at energies far above those accessible to accelerator-based experimentation. The apparatus consists of an array of scintillation counters that detects charged particles produced in the EAS that reach the ground. Here one is utilizing experimental techniques developed in nuclear and particle physics to explore new and exciting science in related areas.

The CYGNUS detector has 204 scintillation counters deployed around the LAMPF beam stop. The counters are spread out over an area of $-8 \times 10^{4} \mathrm{~m}^{2}$. By measuring the relative times at which the counters are struck, the direction of the particle initiating the shower can be determined. Most EAS are produced by primary protons and nuclei. Charged particles bend in the interstellar magnetic fields and so do not point back to their source; only neutral, stable particles, such as photons, point back to their source. Hadron-initiated showers should contain many more muons than photon-initiated showers. The number of muons in the EAS is sampled with the MWPCs from the neutrino detector formerly used in LAMPF Experiment E225, scintillation counters in the E645 tunnel, and scintillators buried underground. Figure IV-7 shows a schematic view of the CYGNUS detectors. CYGNUS started operation in 1986, albeit with a smaller number of detectors at the beginning, and

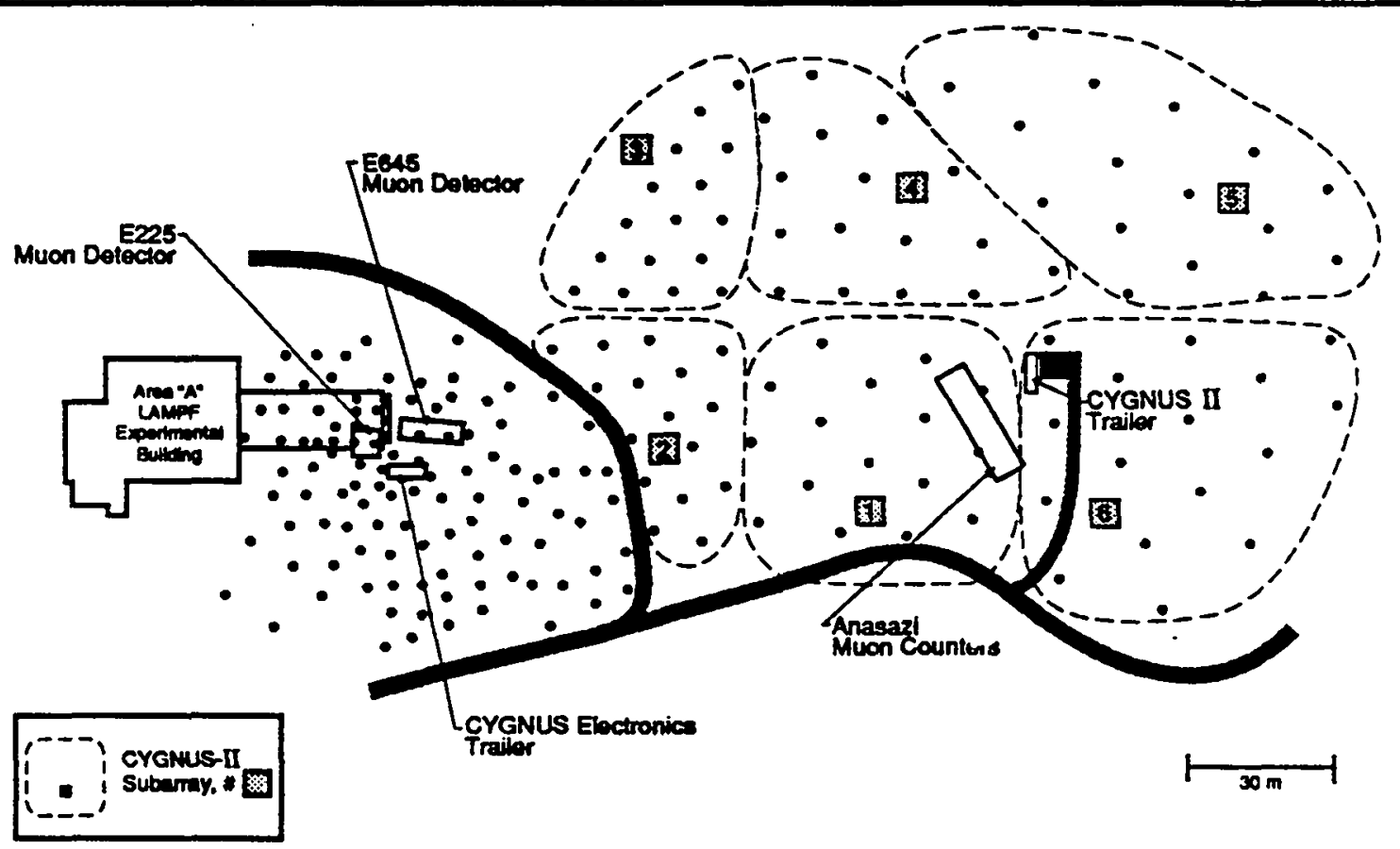

Fig. IV-7. A schematic view of the CYGNUS array at the east end of LAMPF. The dots represent individual scintillation counters. 
has been in continuous operation since. The CYGNUS collaboration now includes scientists from the University of California Berkeley, University of Califomia Irvine, University of California Riverside, University of California Santa Cruz, George Mason University, Los Alamos National Laboratory, University of Maryland, and University of Notre Dame.

Locating this experiment at LAMPF has been particularly cost-effective because:

(1) The high altitude (7000' above sea level) results in sensitivity to incident gamma rays with energies about a factor of four lower than an identical detector placed at sea level.

(2) Large, shielded detectors (the veto shields for LAMPF neutrino experiments) are available to sample the number of muons contained in each shower.

(3) Large quantities of scintillator were available, both from a defunct cosmic-ray experiment in southern New Mexico, and from a dismantled LAMPF neutrino experiment.

Observations over the past 6 years have shown that the compact $\mathrm{X}$-ray sources, Cygnus X-3 and Hercules X-1, are also sources of UHE gamma rays. The measured period for UHE gamma rays from Hercules $X-1$ is significantly different from the period measured with $x$-rays, indicating that UHE gamma rays and $x$-rays are produced in different regions in the source. Surprisingly, the observations also show that the gamma-ray interactions in the atmosphere produce an order of magnitude more muons than expected. Either the nature of photon interactions changes dramatically at these extremely high energies, or the primary particle is some hitherto unknown light, neutral particle; either of these possibilities indicates exciting, new physics.

The CYGNUS experiment has observed the shadow of the sun and the moon in UHE cosmic rays at a level of $>6$ standard deviations, as shown in Fig. IV-8. This technique for measuring the angular resolution of an air shower detector was first proposed over 30 years ago. The CYGNUS experiment is the first to obtain a large enough data set with good enough angular resolution to observe this effect. This measurement shows that the angular resolution is $-0.65^{\circ}$, and that the absolute pointing error is small.

Recently, the large CASA air shower array, located at Dugway, Utah, came on line: the close proximity of CYGNUS and CASA gives rise to the possible simultaneous detection by independent groups of a single UHE gamma-ray burst. Such an occurrence is needed to verify the anomalous nature of previous UHE observations of episodic sources.
There is presently no instrument in the world capable of observing cosmic gamma rays between 1 and $100 \mathrm{TeV}$. This unexplored energy regime appears particularly interesting because one source (the Crab nebula) has been established as a constant emitter of $1-\mathrm{TeV}$ photons by optical telescopes that observe the Cerenkov light produced in EAS. Furthermore, several sources seem to emit bursts of $1-\mathrm{TeV}$ gamma rays more frequently than UHE gamma rays. The air shower technique can be extended to lower energies if a larger fraction of the total area of the array is covered with detectors; the CYGNUS array has detectors covering only $0.5 \%$ of the total array area.

It appears that the most cost-effective way of accomplishing this is with a water Cerenkov detector. The CYGNUS group, augmented by an expanded collaboration, has proposed such a detector, called MIILAGRO, to detect cosmic gamma rays from below 1 TeV to several PeV. MILAGRO would be built in an existing large, covered, water pond located at an elevation of $8700^{\circ}$ located in the Jemez mountains near Los Alamos. Some engineering concepts and read-out electronics designs can be shared between MILAGRO and the LAMPF neutrino program . The CYGNUS scintillation detectors would be moved to become part of Mil.AGRO.

In anticipation of MILAGRO, the CYGNUS group has installed five, 24'-diameter swimming pools within the CYGNUS array, each instrumented with large photomultipliers (PMTs). The information from the PMTs in the pools is recorded for each EAS that triggers

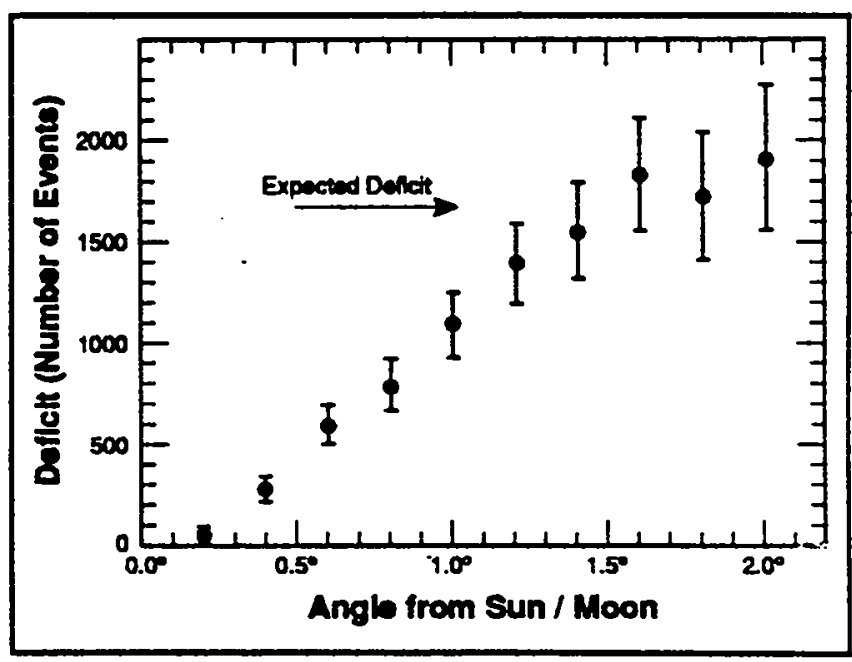

Fig. IV-8. The deficit in the numb:r of events as a function of the angular distance from the center of the sun or moon from the CYGNUS experiment. The shadow of the sun and moon should result in a deficit of about 1600 events. 
the CYGNUS array. The PMTs in the pools detect nearly every shower. In addition, the PMTs register the arrival time of the shower front with high precision so that the angular resolution can be improved by nearly a factor of two. This will markedly improve the sensitivity of CYGNUS to point astrophysical sources.

\section{Measurement of Neutrino Capture Cross Section on $37 \mathrm{Cl}$ and $127 \mathrm{I}$}

One of the great puzzles in science is the discrepancy between the number of neutrinos believed to be produced in the sun and the number detected on earth: this is the "solar neutrino problem." For the past twenty years the Homestake experiment, a radiochemical experiment that detects neutrino capture on ${ }^{37} \mathrm{Cl}$, has measured a capture rate that is about a factor of three below that expected for the standard solar model. More recently, the solar neutrino deficit has also been observed by the Kamiokande water Cerenkov detector and by the SAGE gallium experiment. The favored theoretical explanation at present is the existence of matter-enhanced neutrino oscillations, which requires both non-zero neutrino mass and mixing between neutrino types. In addition, the chlorine experiment may have evidence that the neutrino flux varies with the solar cycle. LAMPF experiment E1213, which will begin running in 1992, will measure the cross section for $\mathrm{v}_{e}$ capture on ${ }^{37} \mathrm{Cl}$ with an anticipated accuracy of $10-15 \%$. This will serve to check the calibration of the Homestake experiment.

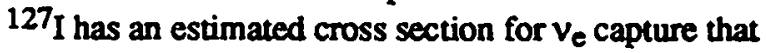
is a factor of 5-10 larger than that for chlorine. An experiment utilizing iodine instead of chlorine has been proposed as an effective way to study the possible correlation of solar neutrinos and the solar cycle. E1213 will also measure the cross section for $v_{e}$ capture on $127_{I}$, which will determine whether an iodine solar neutrino experiment is feasible.

\section{Detection of Neutrinos from Supernovae}

The LSND neutrino detector will be sensitive to bursts of neutrinos from supernovae within our galaxy. Approximately 50-100 events should be detected in a 10-s interval by using a low energy threshold ( $<5 \mathrm{MeV}$ ). Because the detector is mainly mineral oil, most of the observed events will be from the reaction $\bar{v}_{e} p \rightarrow e^{+} n$, where both the positron and the neutron will be detected. The experiment is also sensitive to neutrinos of all flavors through the reaction $v C \rightarrow V C^{*}$, followed by the emission of a $15.11-\mathrm{MeV}$ photon from the decay of the excited state of carbon. LSND will begin data-taking in 1993.

\section{Measurement of Cross Sections of Astrophysical Interest}

Much of our understanding of stellar evolution, stellar dynamics, and nucleosynthesis rests on our knowledge of nuclear physics. Nuclear decay rates and production cross sections form crucial input to theoretical models. Cross sections for a number of processes of astrophysical interest have been measured at LAMPF, including $v_{e} d \rightarrow$ $\mathrm{pe}^{-} \mathrm{p}$, which is the inverse of an important reaction powering the sun, proton-induced spallation of light nuclei, which is needed to understand the abundance of several light elements that are not copiously produced in nucleosynthesis, and a variety of low-energy neutron processes on radioactive nuclei.

The proposed radioactive beams facility at LAMPF will allow the measurement of a large number of cross sections involving nuclei far from stability, and studies of the structure of these nuclei. Reliable information concerning nuclear reaction rates and nuclear structure far from stability is essential for an understanding of nucleosynthesis. The known elemental and isotopic abundances can then be used to pin down the stellar site, environment, and time scales of various processes that play major roles in stellar nucleosynthesis.

\section{Thin-Target, He-Jet System}

\section{Introduction}

Radioactive beam research continues to grow and captivate the interests of the nuclear science, nuclear astrophysics, atomic physics, and materials science communities. Adding to this excitement is the white paper entitled the "The IsoSpin Laboratory (ISL) Research Opportunities with Radioactive Nuclear Beams" that was recently published by the North American Steering Committee for the ISL. Herein the idea of producing intense, high-purity, radioactive beams using the two-accelerator (ISOL/post-acceleration) approach was advanced. This white paper contained a far-reaching discussion of the science that such a next-generation radioactive beam facility would make possible, outlined a benchmark facility based on existing technology, and defined important R\&D issues that should be addressed. A separate section, "The IsoSpin Laboratory at the Los Alamos Meson Physics Facility," briefly summarizes the scientific case for the ISL and highlights the active role 
that Los Alamos is playing in support of the ISL initiative.

Among the R\&D issues raised in the ISL white paper is the feasibility of a high primary beam intensity, thintarget option for the production and rapid release of nonvolatile elements that are not likely to be efficiently released from the standard approach involving thick targets. LAMPF is well suited to address this R\&D question, given the high proton beam intensity of the LAMPF accelerator, the possibility of mounting a thin target, He-jet system relatively quickly and inexpensively in the main beam stop area, and previous experience with He-jet, mass separator systems. In this section, $R \& D$ plans for the thin-target, He-jet option and a number of physics experiments that are being planned using this system are described.

\section{A Thin-Target, He-jet System at the LAMPF Beam Stop}

The experimental proposal to mount a thin-target, He-jet system at the main beam stop area of LAMPF was approved in August 1991. The present design of the Hejet target cell consists of a water cooled, double walled cylinder $(-5 \mathrm{~cm} \phi)$ in which several thin targets (each $-10 \mathrm{mg} / \mathrm{cm}^{2}$ thick) of Th or $U$ are mounted. A buffer gas of He is flowed between the targets to stop the reaction recoil products. Once thermalized, they become attached to large salt clusters introduced into the buffer gas and are then rapidly entrained in and transported through a set of capillary tubes to an experimental area located some 5 to $10 \mathrm{~m}$ away from the target cell. Relatively large transport efficiencies (20 to 80\%) and short transit times (on the order of $1 \mathrm{~s}$ or less) have been routinely achieved in such He-jet systems. The challenge is to see if such a system can be made to work reliably at the LAMPF intensities of $\sim 800 \mu \mathrm{A}$. If so, reasonably large intensities $\left(10^{6}\right.$ to $10^{8} \mathrm{part} / \mathrm{s}$ ) of nonvolatile radioactive species, produced in $800-\mathrm{MeV}$ proton-induced fission, fragmentation, and spallation reactions, will be made available for study.

\section{Research Program}

Several physics experiments that use the He-jet are now being planned. Two nuclear structure investigations are already approved. One involves the use of a fast radiochemical separation system to isolate the most neutron-rich isotopes of $\mathrm{Tc}, \mathbf{R u}, \mathbf{R h}$, and Pd. These nuclei fall directly between the highly deformed nucleus ${ }^{100} \mathrm{Zr}$ and the doubly-magic spherical nucleus ${ }^{132} \mathrm{Sn}$; as such, they are considered to be transitional in nature and an understanding of their nuclear structure is of high interest. The other experiment will determine the magnetic moments of several of the longer lived, neutronrich isotopes in this region with the off-line nuclear orientation method. Samples would be collected with the He-jet and then shipped to the low temperature nuclear orientation facility at the HHIRF in Oak Ridge for measurement.

An exciting experiment that is now being considered is a measurement of atomic parity nonconservation (PNC) transition amplitudes in several radioisotopes of Cs (and possibly Fr) as a high precision test of the standard model of the electroweak interactions. The basic idea behind the experiment is to collect Cs radioisotope samples using the He-jet system, vaporize the samples on-line, selectively capture the Cs isotopes in an optical trap, laser cool the atoms, and transfer them into a second optical trap where finally their PNC amplitude is measured. The atomic measurement itself involves pumping the PNC-violating 6S-7S transition with polarized green laser light in the presence of static electric and magnetic fields (to remove the $F$ and $M$ level degeneracies). The population of the $7 \mathrm{~S}$ state is detected via infrared florescence that occurs when it decays back through the 6P state to the ground state. By suitable reversals of the laser polarization and the electric and magnetic fields, the PNC transition amplitude can be extracted. The result when this amplitude is combined with the appropriate atomic matrix elements is a determination of the weak charge, $\mathrm{Qw}$, for each Cs isotope. By taking a ratio of these measurements for several isotopes the uncertainties arising from the atomic matrix element calculations can be dramatically reduced resulting in a $Q w$ determination at the level of $-0.1 \%$. As such, this elegant atomic and nuclear physics experiment would be particularly valuable in determining the size of the isospin-conserving radiative corrections in the electroweak interaction.

The accuracy of a Cs experiment presently operating at Colorado is a few percent. Already, it is severely constraining extensions of the standard electroweak theory, in fact, more so than any other measurements apart from those at LEP. The combination of this experiment with the mass of the $Z^{0}$ from LEP represents the most stringent test of the standard model at this time.

The success of the titin-target, He-jet system is an important R\&D demonstration for the ISL. The goal is not only to demonstrate the attractiveness of this technology, but to undertake key physics experiments as our capabilities evolve. The outside user interest in this program is growing with the participation of some 20 scientists from the U.S. and Europe. 


\section{Facilities}

\section{Introduction}

The research carried out at LAMPF requires sophisticated facilities and capabilities, along with a broad technological base. It is essential that LAMPF have a strong program of experimental support to allow experiments of the highest scientific quality. Users play major roles in building new facilities in addition to collaborating on experiments.

The $\mathrm{H}^{+}$beam in Line $A$ is used to produce highquality secondary beams of pions, muons, neutrinos, and spallation products. A test channel in Area A provides a moderate quality beam of charged particles for development and testing of experimental apparatus. A neutrino research area and facilities for isotope production and radiation-damage studies are provided at the Line $A$ beam stop. The polarized $\mathrm{H}^{-}$beam is used together with the Nucleon Physics Laboratory (Areas B and C) for nucleonnucleon and nucleon-nucleus studies. The $\mathrm{H}^{-}$beam is also used for atomic beam studies at HIRAB. The unpolarized $\mathrm{H}^{-}$beam is used to fill the Proton Storage Ring to deliver beam to the Los Alamos Neutron Scattering Center.

\section{Energetic Pion Channel and Spectrometer (EPICS)}

The Energetic Pion Channel and Spectrometer (EPICS) is a high resolution magnetic beam line and spectrometer facility covering an incident pion energy range of 100 to $300 \mathrm{MeV}$. The high-energy resolution and incident flux of pions are unique in the world. A momentum dispersed beam $( \pm 1 \%)$ of up to $2 \times 10^{8} \pi^{+} / \mathrm{s}$ is directed onto a $20-\mathrm{cm}$ (vertical, dispersion direction) by 10-cm (horizontal, scattering direction) scattering target. Scattered particles are detected by a 10-msr, Q3D2 spectrometer. After the quadrupole triplet a set of $x-y$ position sensitive wire chambers image the target, enabling software reconstruction for determining incident momentum and providing background rejection. Energy resolution is typically $140 \mathrm{keV}$ at $180 \mathrm{MeV}$ for inelastic scattering studies with thin targets. The standard spectrometer configuration covers an angular range of -10 to $+120^{\circ}$. With the addition of a circular pole magnet at the scattering target, effective outgoing scattering angles of $180^{\circ}$ can be reached. A circular pole magnet with a $10^{\circ}$ bend angle can be positioned just downstream of the scattering target to "sweep out" the incident pions, enabling the oppositely charged outgoing pions in double charge-exchange reactions at scattering angles of $5^{\circ}$ to be detected.

Much of the physics program has involved $\pi^{ \pm}$elastic scattering and the excitation of low-lying states. Questions of neutron and proton distributions, natural and unnatural parity excitations have been addressed. Isospin mixing between states has been observed and studied. Shell-model studies of stretched states of high spin with simple configurations have been pursued. The $(\pi, p)$ reaction on several nuclei was observed. Elastic and inelastic scattering has been pursued at angles back to nearly $180^{\circ}$. The successful use of large quantities of gaseous tritium $(200 \mathrm{kCi})$ has allowed scattering of $\pi^{ \pm}$ from the $A=3$ system to examine differences between neutrons and protons. Pion double-charge-exchange (DCX) has been intensively investigated in terms of exploring reaction mechanisms and nuclear structure, as well as a means of measuring masses and binding energies of exotic light nuclei. Of particular note in recent years is the activity in measuring and characterizing giant dipole resonance (GDR) states built on excited states. The EPICS capabilities are unique in enabling the observation of resonances of considerable width located at high excitation energy, and superimposed on a large background from the continuum of states. Pion inelastic scattering 
asymmetries have been successfully measured on polarized ${ }^{13} \mathrm{C}$ targets. The resolution of EPICS allowing one to help separate the backgrounds associated with these complicated targets.

\section{Large Acceptance Spectrometer (LAS)}

The Large Acceptance Spectrometer (LAS) is a portable, medium-resolution magnetic spectrometer that has been used for studies in the pion program using both the $P^{3} E$ and LEP secondary beam channels. It is instrumented with wire chambers to enable full path reconstruction through the spectrometer, and a Cerenkov detector to distinguish pions from background electrons. With a medium length flight path $(6-7 \mathrm{~m})$, the spectrometer is suitable for many experiments with incident pion energies from 100 to $550 \mathrm{MeV}$, typically with an energy resolution of $1.5 \mathrm{MeV}$.

The spectrometer is unique in the pion program, due to the large number of configurations in which it may be used and the high pion energies which are available at the $\mathrm{P}^{3}$ channel. The minimum configuration consists of two quadrupole magnets and a dipole magnet ( $45^{\circ}$ bend), with detectors placed before and after the dipole magnet. Variations which have been used include: (1) placing a Cmagnet just downstream of the scattering target to sweep away incident pions, similar to the technique employed at EPICS. This option is used to decrease background associated with the beam in small angle DCX studies. (2) decreasing the bend angle to $30^{\circ}$, yielding a momentum acceptance of $-15 \%$ through $+20 \%$ compared to the usual acceptance of $-8 \%$ through $+10 \%$. (3) placing a strong magnet (1.5 T) at the target location, for polarized target studies. This is used in conjunction with a simple mechanical mechanism for varying the lateral displacement of the spectrometer magnets, to maintain the optimum spectrometer acceptance. (4) using other detectors in coincidence with LAS, such as large BGO crystals.

Due to the ease of operation of the spectrometer, its great versatility, and its good performance, LAS has been used in many different kinds of experiments. Initially, it was used for high-energy (above the delta resonance) DCX studies to study reaction mechanisms for transitions to the double isobaric analog state (DIAS). Elastic data were taken to complement these studies. More recently, the DCX reaction mechanism studies have been extended to the continuum, detecting protons in coincidence with a pion in the LAS. Again, as in the DIMS case, the corresponding non-charge-exchange data (here, quasielastic scattering) have been taken. LAS has been successfully used at the LEP channel to detect elastically scattered pions from polarized ${ }^{13} \mathrm{C}$ at and below resonance energies. Future experiments will use LAS at $\mathrm{P}^{3}$ along with a polarized ${ }^{3} \mathrm{He}$ targets to study both elastic scattering asymmetries and also scattering in the quasifree region.

\section{Scruncher/Clamshell}

The Scruncher is a superconducting radio-frequency cavity, used to change the longitudinal phase space of the pion beam at LEP. It became operational in 1990 and is the only device available in the meson physics community for increasing the flux of a secondary beam without decreasing the energy resolution. The cavity rotates the beam in momentum-time space, to increase the flux in a given momentum bite. It relies on the initial narrow time structure ( $<50 \mathrm{ps)}$ of the primary proton beam, which is only available at LAMPF. It is the first application of superconducting If technology at LAMPF. By the nature of the device, its performance characteristics are very dependent on the application. It may be used to momentum compact the LEP pion beam with energies in the range 30 to $120 \mathrm{MeV}$. It has been used, in conjunction with the Clamshell spectrometer. Good resolution $(\sim 300 \mathrm{keV})$ experiments at $50 \mathrm{MeV}$ have achieved a net gain in pion count rate of over four.

The Clamshell is a large-momentum acceptance $( \pm 15 \%)$ magnetic spectrometer optimized for detecting low energy pions ( $<80 \mathrm{MeV}$ ). It is used exclusively at the LEP channel. The length of the flight path $(1.8 \mathrm{~m})$ through the spectrometer is minimized by incorporating focusing components in the design of the dipole magnet, rather than adding extra magnetic elements to the system. A set of wire chambers, scintillators, and absorbers is placed at the exit of the spectrometer to facilitate path reconstruction through the spectrometer and particle identification. When the Clamshell is used with the Scruncher, a position-sensitive wire chamber is provided at the entrance to the spectrometer to improve particle tracking through the magnet. A sweeping magnet can be added just downstream of the scattering target to enable DCX experiments at small angles (25 at low energies).

Programs using the Scruncher/Clamshell include studies of identifying states in DCX from medium-mass nuclei and systematic studies of the DIAS in the nickel isotopes to study effects of shell-model correlations in the nuclear wave function. With the addition of the Scruncher, it is now feasible to measure low-lying states in nuclei such as ${ }^{10} \mathrm{~B}$, where the cross sections are small and the resolution requirements are stringent $(-300 \mathrm{keV})$.

The Scruncher will play an important role in the physics program for the Neutral Meson Spectrometer. In particular, accurate single charge exchange cross sections 
for the pion-nucleon reaction must be performed at low energies, where the Scruncher will significantly reduce the beam time requirements, without compromising the resolution required for background subtraction.

\section{Bismuth Germinate Ball}

The Bismuth Germinate Ball (BGO ball) is a nearly $4 \pi$ sr detector with moderate energy resolution. It consists of 30 phoswitch detectors, each detector having a 3-mm-thick plastic scintillator optically coupled to the front of a 5.6-cm-thick bismuth germinate (BGO) crystal with a 7.62-cm-diameter photomultiplier tube mounted on the back. Both the fast $(\Delta E)$ and slow $(E)$ signals from the individual detectors are analyzed to distinguish between particles such as pions, protons, deuterons, gamma rays, and neutrons. The BGO crystals are thick enough to stop $185-\mathrm{MeV}$ protons and $90-\mathrm{MeV}$ pions. The total solid angle covered by these crystals is $0.88 \times 4 \pi$ sr and span scattering angles from 26 to $161^{\circ}$. Each individual detector is supported in a 0.5 -mm-thick electro-formed nickel can, which has a 0.05 -mm-thick entrance window, with the support covering about $5 \%$ of the solid angle of each crystal.

Since the completion of the BGO ball, cross sections were measured for the emission of protons following $\pi^{+}$ absorption on a range of targets from the deuteron to ${ }^{238} \mathrm{U}$ for pion kinetic energies from 17 and $500 \mathrm{MeV}$. These data are the first of their kind in the sense that there was little restriction on the correlations of the outgoing particles. These data also provided the first direct measure of the cross section for non coplanar three-proton emission for a variety of nuclei at several energies. The BGO ball is also used to measure inclusive single-chargeexchange cross sections. An upcoming experiment will systematically measure the two-proton component of the pion absorption reaction on various nuclei between 30 to $150 \mathrm{MeV}$ in $10-\mathrm{MeV}$ steps.

In the future, the BGO ball will be used for feasibility studies of experiments in which deeply bound pionic atoms could be formed. Measurements of the shifts and widths of the levels of the deeply bound pionic atoms can be used to determine the real and imaginary parts of the low energy $\pi-\mathrm{N}$ potential. The BGO ball is an ideal detector for such studies, since it can detect all of the outgoing particles. This will help in the understanding of how to select events associated with the pionic atoms, by using patterns of outgoing particles that are characteristic of the formation and the deray of the bound pionic state.

\section{Neutral Meson Spectrometer}

A Neutral Meson Spectrometer (NMS) is being constructed to detect neutral pions and etas. Using modem materials and design, a factor of ten improvement in resolution and a doubling of the solid angle of the existing $\pi^{0}$ spectrometer is attainable. Both the NMS and the existing $\pi^{0}$ spectrometer are unique instruments, with no other other instrument in the world even approaching their capabilities. There is a queue of approved experiments that would consume several years running time. A broad range of pion-nucleus and pion-nucleon reaction studies over the full spectrum of pion energies available at LAMPF is addressed. A partially complete NMS will be used in the summer of 1992 to make measurements of pion single charge exchange (SCX) on the deuteron and on the proton.

Each arm consists of four planes, roughly $60 \mathrm{~cm}$ high and $100 \mathrm{~cm}$ wide, of bismuth germinate (BGO) in which the photons are converted into charged particles. Each plane is followed by cathode readout wire chambers for $x-y$ position information. The short radiation length of BGO $(1.1 \mathrm{~cm})$ is needed for maintaining the required photon vertex resolution $(250 \mu \mathrm{m})$. The total energy of the shower is measured by a calorimeter of CsI(pure). This material was chosen for its short radiation length $(1.8 \mathrm{~cm})$ and fast response time. The statistical component of the energy resolution for gamma rays of energy $100 \mathrm{MeV}$ and above will be less than $1.0 \%$. These calorimeters are unique in their quality as high energy electromagnetic detectors.

The high count rate capability and the large solid angle of the NMS will enable an accurate probe of fundamental processes related to the extraction of the $\sigma$ term in QCD from the pion-nucleon scattering amplitudes. The resolution of the NMS will allow the identification and study of many states previously not resolved by the existing $\pi^{0}$ spectrometer. SCX reactions exhibit special selection properties to $T=1$, natural parity states. The $\Delta$ can be studied via isovector spin-flip excitations, which are found to exhibit greater sensitivity to the potential felt by the intermediate $\Delta$ than isoscalar or non-spin-flip transitions. The role of sequential SCX in double charge exchange is not well understood, requiring the identification and measurement of many excited states in SCX, before the role of exotic single step processes can be inferred. 
The Optically Pumped Polarized Ion Source (OPPIS)

The Optically Pumped Polarized Ion Source (OPPIS) provides polarized $\mathrm{H}^{-}$beam. In 1991 operation, it provided $38 \mu \mathrm{A}$ of beam with a polarization of 0.64 and an overall source availability of $90 \%$. OPPIS development was started in 1986 to replace the Lamb-shift source and increase LAMPF's polarized beam capability by a factor of ten. The present OPPIS $P^{2} I$ figure of merit is a factor of $\mathbf{3 0}$ greater than the Lamb-shift source. The source was developed in close collaboration with similar development projects at KEK, INR, and TRIUMF. The LAMPF development effort was innovative in several aspects of the ion source design: the use of an ECR $\mathrm{H}^{+}$ source to alleviate the emittance growth problems associated with charge exchange in a high magnetic field; the use of $\approx$ high magnetic field from a superconducting magnet to increase the polarization transfer, the use of higher intensity, more reliable $\mathrm{Ti}$-sapphire lasers to optically pump a potassium vapor in the polarizer cell; the development of the accel-accel ECR extraction geometry that has greatly increased the polarized intensity at LAMPF, KEK, and TRIUMF. The polarized beam injector also includes an efficient system to produce chopped beam for neutron time-of-flight experiments with minimal loss of beam current. The high level of performance and availability of this new source, coupled with the fact that linacs have no depolarization resonances, and LAMPFs unique ability to provide both polarized and unpolarized beam simultaneously, make LAMPF an outstanding polarized beam facility. In fact, LAMPF provided more polarized proton beam for physics experiments last year than any other major facility in the world.

\section{Neutron Time-of-Flight Facility (NTOF)}

The Neutron Time-of-Flight (NTOF) facility was designed to study $(p, n)$ reactions in the energy range from 200 to $800 \mathrm{MeV}$. It combines a wide energy acceptance (several hundred $\mathrm{MeV}$ ) with good energy resolution $(600 \mathrm{keV})$, large momentum transfer range $\left(3 \mathrm{fm}^{-1}\right)$, and full polarization analysis of the neutron flux. This unique combination of capabilities allows a wide range of physics issues to be addressed, ranging from studies of discrete final states to excitation of the delta in the nuclear medium.

The prinary components are a magnetic beam swinger and neutron precession system consisting of seven dipole magnets and a superconducting solenoid, a target chamber that can accept normal targets on a ten- position target ladder, cryogenic targets, or polarized targets, a paved neutron flight path that extends to $620 \mathrm{~m}$, and a movable detector station housing a neutron time-offlight detector. The detector system consists of four largevolume position-sensitive scintillation detectors configured as a neutron polarimeter. The system is continuously monitored and calibrated using cosmic-ray muons and can routinely maintain an intrinsic timing resolution of better than 300 ps. A unique capability at LAMPF that greatly improves the energy resolution of NTOF at incident energies less than $800 \mathrm{MeV}$ is a "rebunching" technique. In this operation, certain of the accelerator tanks past the last accelerating module are excited with if at a phase that produces no net acceleration but with amplitude that skews the particle distribution in energy and phase so that the micropulse is focused in time when it reaches the neutron detector.

NTOF is currently the only $(p, n)$ facility in the world with full polarization transfer capabilities. It is also the only facility with the ability to study the important delta region of excitation. The efficiency, energy resolution, and acceptance exceed the capabilities of the TRIUMF facility, and only IUCF can match the resolution obtained, but without polarization transfer and without the higher energy beam required to access the quasifree and delta regions of excitation.

The NTOF facility became fully operational in 1990 with the completion of the high-intensity polarized ion source (OPPIS) and many important, long-anticipated studies of isovector nuclear structure and spin observables are just beginning. Measurements of polarization observables for transitions to discrete final states over a wide angle range with good resolution provide information about very specific pieces of the effective nucleon-nucleus interaction. Such measurements also provide unique tests of the pionic component of modern relativistic reaction models. Polarization analysis allows an experimental extraction of isovector spin-longitudinal form factors; these nuclear structure functions, which directly involve the fundamental pionic interaction, cannot be measured in electron scattering. Furthermore, for studies of isovector continuum strength, either in quasifree scattering, the giant resonance region, or the delta region, the purely isovector $(p, n)$ reaction has distinct advantages over similar probes such as (p,p), and is simpler to interpret than other more complex probes such as $(d, 2 p)$ or $\left({ }^{3} \mathrm{He}, t\right)$.

\section{High Resolution Spectrometer (HRS)}

The High Resolution Spectrometer (HRS) is the highest resolution (60 keV at $800 \mathrm{MeV}$ ) proton spectrometer at intermediate energies. With a solid angle of $3 \mathrm{msr}$ 
and a momentum acceptance of $\pm 1 \%$, the HRS is an ideal tool for studying low-lying discrete states. The angular resolution of $2 \mathrm{mrad}$ makes careful angular dependence measurements a powerful tool for nuclear structure studies. The energy range of 200 to $800 \mathrm{MeV}$ allows energy-dependent studies, which may be cross checked at IUCF and TRIUMF.

Coupled with the three spin projections possible from the incident polarized proton beam, the focal plane polarimeter (FPP) enables a full determination of spin transfer coefficients. These coefficients enlarge the experimental testing ground for nuclear structure and reaction mechanism theories. In most cases, all spin transfer coefficients can be measured, and from these fundamental measurements nuclear response functions can be inferred.

The spectrometer facility has been successfully used with polarized proton, deuteron, and ${ }^{13} \mathrm{C}$ targets. Along with the complete spin measurement capabilities, the good resolution enables excellent background rejection. A development program is underway to provide small volume, compact polarized targets that are user-friendly and easy to install.

\section{Medium Resolution Spectrometer (MRS)}

The Medium Resolution Spectrometer (MRS) is a good resolution ( $1 \mathrm{MeV}$ at $800 \mathrm{MeV}$ ) proton spectrometer with a very large momentum acceptance $( \pm 20 \%)$. Complete spin transfer measurements can be done with the polarized beam capabilities and the focal plane polarimeter in the detection system. The net bend angle of the dipoles was carefully designed to be only $18^{\circ}$, such that over the full energy range ( 300 to $800 \mathrm{MeV}$ ) the outgoing proton spin is not precessed to have a significant longitudinal component in the focal plane detectors. The ability to measure spins over the full energy range (and at high excitation energies) and the large momentum acceptance makes the MRS ideally suited to spin transfer measurements in the continuum, including the excitation of the delta resonance.

The MRS can easily be moved to the polarized neutron beam in Area BR. In this mode, wire chambers are placed downstream of the scattering target to project back to the target and image the larger beam spot, preserving the intrinsic resolution of the spectrometer system and enabling detection of low-lying nuclear states. Also, the higher beam energies available at LAMPF enable studies in the delta excitation energy region, which are more extensive than those possible at TRIUMF.

Plans are being made to move the MRS to the HRS dome in Line C. At present, for worst-case accident scenarios of dumping the full power of the intense (1-mA) $\mathrm{H}^{-}$beam (used for Line $\mathrm{D}$ under normal operations), the radiation shielding is inadequate. In the HRS dome, this is not the case. The MRS could take advantage of the increased shielding to implement its full physics program, as well as open up the possibility of dual-arm experiments with the HRS.

\section{Polarized Neutron Area}

BR is a low- to medium-resolution high-rate polarized neutron facility. It is used for np elastic and inelastic experiments, few-nucleon experiments (e.g., nD), and nuclear physics $A(n, p)$ experiments such as the study of the isovector monopole resonance in ${ }^{90} \mathrm{Zr}(n, p)$. Presently approved A-priority experiments will extend through 1995.

Following the completion of the Optically Pumped Polarized Ion Source (OPPIS) in 1990, this facility now produces $5 \times 10^{7}$ neutrons $/ \mathrm{sec}$ at a polarization of 0.5 . The neutron beam energy is variable from 300 to $800 \mathrm{MeV}$. Beam polarization is measured to \pm 0.01 by two proton polarimeters and a neutron polarimeter. Full spin-precession capability allows the neutron spins to be oriented in any direction.

The nucleon-nucleon physics program has taken advantage of several available capabilities including large volume cryogenic (15-liter liquid hydrogen) and polarized targets, two large acceptance spectrometers, and a highefficiency neutron detector. Development and testing is under way to implement a charged particle cylindrical $>2 \pi$ sr detector for the study of the $\mathrm{np} \rightarrow \mathrm{pp} \pi^{-}$reaction.

Since a removable wall separates the proton beam line for MRS and the neutron beam line (BR), one can easily move the spectrometer on air pads between the two areas. The neutron source for $(n, p)$ experiments is usually a thin lithium target producing a nuclear physics grade neutron beam with an energy width of less than $1 \mathrm{MeV}$ and neutron fluxes of $10^{5} \mathrm{n} / \mathrm{s}$.

\section{Polarized Targets}

A polarized target is a cryogenic system used to polarize the nuclei of target materials for scattering experiments. The polarized target capability has gieat versatility and targets have successfully been provided for pion, proton, and neutron beam experiments. The pion and neutron beam targets have required the development of large volume targets to accommodate the large beam spots. The target nuclei are polarized by the method of Dynamic Nuclear Polarization (DNP). With DNP, target material containing the nuclei of interest is cooled to low 
temperature $(<0.5 \mathrm{~K})$ in a cryostat, exposed to a strong 2.5-T field, and polarized by applying microwaves at a frequency near $70 \mathrm{GHz}$. Target polarization is measured by NMR. A frozen spin target requires a dilution cryostat to cool the target material to very low temperature $(<0.1 \mathrm{~K})$ so the polarization can be maintained at lower field $(<0.5 \mathrm{~T})$. LAMPF has four target cryostats and four magnets plus other equipment needed for two complete target systems and most of the equipment for a third. LAMPF can provide polarized targets with a target volume up to about $65 \mathrm{cc}$ with vertical $(\mathrm{N})$, longitudinal (L), or horizontal (S) orientation for the spin direction. Frozen spin targets with $\mathrm{N}, \mathrm{L}$, or $\mathrm{S}$ spin direction can be provided. In 1988, LAMPF performed its first experiment with a polarized nuclear target $\left({ }^{13} \mathrm{C}\right)$. LAMPF can provide polarized targets of the following light nuclei: ${ }^{1} \mathrm{H}_{1},{ }^{1} \mathrm{D}_{1},{ }^{6} \mathrm{Li}_{3},{ }^{7} \mathrm{Li}_{3},{ }^{10} \mathrm{~B}_{5},{ }^{11} \mathrm{~B}_{5},{ }^{13} \mathrm{C}_{6},{ }^{14} \mathrm{~N}_{7},{ }^{15} \mathrm{~N}_{7}$, and ${ }^{19} \mathrm{~F}$.

\section{High Resolution Atomic Beam Facility}

The $800-\mathrm{MeV} \mathrm{H}^{-}$beam uniquely provides one of the most interesting research tools in atomic physics. Using conventional lasers and the large relativistic transformations, one can probe the fundamental atomic systems, $\mathrm{H}^{0}$ and $\mathrm{H}^{-}$, in ways that are simply not possible in any other facility presently available in the world. Laboratory laser beams operating in the near ultraviolet can be Dopplershifted in frequency by a factor of as much as 3.4 into the vacuum ultraviolet to a regime of intensity and resolution that is inaccessible by conventional means, in both the $\mathrm{H}^{0}$ and $\mathrm{H}^{-}$systems. The Doppler-shifted light is continuously tunable over a decade with impressive energy resolution. A feature in the $\mathrm{H}^{-}$spectrum at $10.925 \mathrm{eV}$ has, for example, been delineated with a resolution of $3 \mathrm{meV}$. Further advances in resolution can be anticipated. A study of the hydrogen Balmer alpha line in the microvolt range has been proposed. Laser intensities in the atomic rest frame are increased by a factor as much as 11.6 over laboratory intensities (intensity transforms like the Doppler-shift squared). Thus, the world's most intense laser beam would be a decade more intense in the frame of an $800-\mathrm{MeV} \mathrm{H}^{-}$ion.

In the atom's frame relatively modest magnetic fields appear as ultra strong electric fields. For example, a 1-Tesla lab field becomes $4.7 \mathrm{MV} / \mathrm{cm}$ - by no means a mere perturbation. Combining the intensity enhancement with the Doppler frequency tuning, a unique tool exists for the study of multiphoton processes in both $\mathrm{H}^{0}$ and $\mathrm{H}^{-}$. No other laboratory can tune the frequency of their intense lasers as can be done at LAMPF. Other studies in this rapidly advancing field are confined to a limited number of relatively discrete frequencies.

\section{Time-of-Flight Isochronous Spectrometer (TOFI)}

The time-of-flight isochronous (TOFI) spectrometer is designed to study short-lived, exotic nuclei that are produced in fragmentation, fission, and spallation reactions using the high-intensity $(1-\mathrm{mA}), 800-\mathrm{MeV}$ proton beam of LAMPF. TOFI his revolutionized the measurement of the masses of neutron-rich nuclei. A fraction of these reaction products recoil from a thin target $\left(\sim 1 \mathrm{mg} / \mathrm{cm}^{2}\right.$ located in the LAMPF switchyard) and are captured by a $22-\mathrm{m}$-long transport line in which they are filtered according to their mass-to-charge ratio and are matched to the phase space acceptance of the TOFI spectrometer. The spectrometer itself consists of four identical $80^{\circ}$ bending magnets.

The unique feature of this spectrometer is that the flight time of an ion passing through the system depends only on its mass-to-charge ratio (and not its velocity). Thus a mass determination (at the 10 part per million level) requires only a single high precision time-of-flight measurement. To date, over 80 nuclei have been measured -- most for the first time. A recent development in the use of the TOFI spectrometer is as an ion-tagging device for decay studies. The latter is enabling the development of a $\beta$-delayed neutron and multi-neutron decay experimental program.

\section{Los Alamos Radiation Effects Facility}

The Los Alamos Radiation Effects Facility (LASREF) is located at the Line A beam stop, target station A-6. The large radiation volume and the capability for in-situ measurements, as well as the high fluxes available, make LASREF one of the world's best radiation effects facility. Since 1985, radiation damage and radiation effects studies have utilized the direct proton beam $\left(-2 \times 10^{14} \pi / \mathrm{cm}^{2}-s\right)$ and the neutron flux $(\sim 5 \times$ $\left.10^{13} \mathrm{n} / \mathrm{cm}^{2}-\mathrm{s}\right)$ that are produced from proton interactions in the beam stop. The LASREF facility has been used extensively for radiation damage studies of components and electronics for the large SDC detector for the SSC and will be used for additional studies for components for the GEM detector. A review committee representing the Office of Fusion Energy Research has determined that LASREF is an effective testing facility for fusion reactor ceramic components and diagnostic components. Material scientists from the Japanese Monbusho research team are considering LASREF as a major facility for a long-term 
program of testing and development for their fusion program. The LASREF is expected to play a major role as a test bed for the Accelerator Transmutation of Waste (ATW) and Accelerator Production of Tritium (APT) projects. All of these programs would benefit from increased neutron flux. An ongoing study, which will determine the extent of flux enhancement and spectrum modification possible, is under,way.

\section{The Proton Storage Ring (PSR)}

The Proton Storage Ring (PSR) functions as a highcurrent accumulator and pulse compressor to provide intense pulses of $800-\mathrm{MeV}$ protons for the Los Alamos Neutron Scattering Center (LANSCE) spallation neutron target. The peak intensities are unmatched anywhere in the world. LANSCE neutron beams are used for a wide spectrum of experiments in condensed matter physics, materials science, and nuclear physics. The ring accepts and compresses proton pulses up to $1 \mathrm{~ms}$ long from the linac; the extracted pulse width is approximately $250 \mathrm{~ns}$ base-to-base. Operating at $20 \mathrm{~Hz}$, PSR typically delivers an average current of 70 to $80 \mu \mathrm{A}$; the corresponding peak proton intensity $\left[2.5 \times 10^{13}\right.$ protons per pulse (ppp)] at the LANSCE target is the highest available at any of the accelerator-based facilities currently operating. It is expected that improvements to the beam delivery system, featuring the development of a substantially brighter $\mathrm{H}^{-}$ ion source, will allow attainment of the design intensity, $100 \mu \mathrm{A}$, in 1993-94. Although the neutron scattering community has seen several proposals for similar neutron sources based on a compressor ring injected by a proton linac, to date, only PSR has been constructed. This, coupled with its unmatched peak intensities, gives PSR a unique utility, beyond the research opportunities afforded at LANSCE, as a test bed for the development of the accelerator physics and technology of high-intensity rings. This capability has attracted (and continues to attract) collaborators in accelerator physics studies from KEK (Japanese Hadron Project), TRIUMF (KAON project), Moscow, and CERN. The objects of these studies have included: (1) The novel injection scheme, in which the incoming $\mathrm{H}^{-}$beam is stripped to $\mathrm{H}^{0}$ in a high-field stripper magnet, enters the ring lattice through a dipole, and is stripped to $\mathrm{H}^{+}$in a carbon foil. (2) The development of a minimal-size carbon stripping foil with "massless" carbon fiber suspension. (3) Identification and mitigation of beam loss mechanisms in the ring. (4) A high-intensity, transverse beam instability, now identified tentatively as an e-p instability; while PSR can be operated stably at proton intensities up to $4 \times 10^{13} \mathrm{ppp}$, the instability is of central importance in the design of even higher intensity rings.

\section{The Manual Lujan, Jr. Neutron Scattering Center (LANSCE)}

The Manuel Lujan, Jr. Neutron Scattering Center (LANSCE) is a pulsed spallation neutron source equipped with time-of-flight spectrometers for condensed-matter research. LANSCE yields a higher neutron beam flux than any other spallation neutron source used for condensed-matter research. LANSCE offers a range of instruments for determining the atomic and magnetic structure and dynamics of polycrystalline and crystalline materials, such as high-temperature superconductors, pharmaceuticals, and polymers; and the structure of molecular coatings of solid and liquid surfaces. A powder diffractometer at LANSCE has a higher resolution than any other instrument of its type in the United States.

At LANSCE, neutrons are produced by spallation when a pulsed, $800-\mathrm{MeV}$ proton beam impinges on a tungsten target. The pulses are provided by the LAMPF accelerator and the proton storage ring (PSR), which can alter the intensity, time structure, and repetition rate of the pulses. Spallation produces neutrons of relatively high energy, so moderators adjacent to the target must be used to reduce the energies to those required for condensedmatter research. Because of the unique design of the splittarget, flux-trap moderator, reflector-shield which provides the high neutron beam flux, scientists can perform experiments more quickly, more accurately, and with smaller samples at LANSCE than at any other similar facility.

Research programs at LANSCE cover a broad range: solid-state physics, chemistry, metallurgy, crystallography, biophysics, materials science, and nuclear physics. In particular, the study of time reversal and parity symmetries using epithermal neutrons has been of great importance to the nuclear physics community. The neutrons are moderated in water to obtain an epithermal spectrum $(1-10,000 \mathrm{eV})$. The neutrons are then polarized (40\%) by selective absorption in a cell of polarized hydrogen.

\section{Volume Production Multicusp $H^{-}$Ion Source}

A Volume Production Multicusp $\mathrm{H}^{-}$Ion Source (VPMOUS) is being developed at LAMPF as part of the Proton Storage Ring (PSR) upgrade. VPMHIS has the potential of producing a $30-\mathrm{mA} \mathrm{H}^{-}$beam with a normalized emittance of $0.04 \mathrm{~cm}$-mrad. The Surface 
Production Multicusp $\mathrm{H}^{-}$Ion Source now being used operates at $95 \%$ availability and produces a $16-\mathrm{mA} \mathrm{H}^{-}$ beam with a normalized emittance of $0.08 \mathrm{~cm}$-mrad. The brighter beam is required for PSR to reach its design goal of $100 \mu \mathrm{A}$ average current. It is also a necessary part of new initiatives such as the Pion Linear Accelerator and the Pulsed Lepton Source. This new source is scheduled for installation in 1994.

LAMPF has uniquely qualified personnel and facilities to successfully complete this development project. The LAMPF Ion Source Test Stand is one of the world's most complete test facilities. It includes a $100-\mathrm{kV}$ isolation stand, high-voltage accelerating column, pulsed response $70-\mu \mathrm{a}$ peak $100-\mathrm{kV}$ power supply, $4.5-\mathrm{m}$ flight path, $45^{\circ}$ bending magnet, focusing solenoid, slitcollector emittance station, and multiple current measuring devices. The first significant VPMHIS performance data were taken in collaboration with LBL on this test stand in 1984. LAMPF personnel have ion source development experience from developing the surface production source and OPPIS. Further, their eight years of experience operating a multicusp $\mathrm{H}^{-}$source in a production environment uniquely qualifies them to solve the operational problems of this new source. The development of a production-quality VPMHIS would also be of significant benefit to SSC and BNL. 


\section{Future Facility Plans}

\section{IsoSpin Laboratory (ISL)}

\section{Introduction}

In 1989, the Nuclear Science Advisory Committee stated "Wholly new vistas would be opened by a radioactive nuclear beam (RNB) accelerator." (NSAC Long Range Plan). This statement was one of the outcomes of a year-long planning process conducted by the nuclear science community. The low-energy nuclear science community recommended the building of an RNB accelerator as their highest priority long range project. In support of this recommendation they stated: "The atomic nucleus, comprised of up to a few hundred stronglyinteracting nucleons, is the best quantum system for illustrating the profound relationship between sub-particle, single-particle and collective degrees of freedom." "The strength of low energy nuclear physics is its breadth and its relation to other disciplines, e.g., astrophysics, atomic physics, condensed-matter physics, many-body theory, and quantum chaos." As such, low-energy nuclear physics "is indeed the heart of nuclear physics." (Summary of the Town Meeting on Low Energy Nuclear Physics, April 1718, 1989.)

This enthusiasm for low-energy nuclear physics and the development of an RNB accelerator has continued unabated to the present day through a series of international conferences, and workshops including one hosted by Los Alamos in April 1990 at which a North American radioactive beam steering committee was formed. This committee has recently published "The IsoSpin Laboratory (ISL), Research Opportunities with Radioactive Nuclear Beams, ${ }^{n}$ which describes the initial scientific program that could be undertaken at ISL, and has coordinated the establishment of a user community that now numbers over 400 scientists.
Los Alamos has been a full participant in the development of the ISL. We are now poised to begin both R\&D in support of the ISL (see "Thin-Target, He-Jet R\&D - An Experiment in Support of the ISL Initiative" in this report) and implementation studies of the ISL at LAMPF. This latter effort is motivated by our existing scientific program on exotic nuclei, our nascent RNB physics program, and the existing expertise and facilities at LAMPF. In this article, we briefly review the scientific case for the ISL using examples from the ISL white paper, describe the benchmark facility, and demonstrate LAMPF's central role in the ISL initiative.

\section{The Science of the ISL}

The science of the ISL can be subdivided into two broad categories, low-energy nuclear physics and disciplines using nuclear physics techniques and/or results (i.e., astrophysics, atomic and materials science). A key area of research in low-energy nuclear physics is the study of exotic nuclei with extreme $N / Z$ ratios. These nuclei have unexpected shapes such as the prolately deformed ${ }^{31} \mathrm{Na}$ or unusual matter distributions such as ${ }^{11} \mathrm{Li},{ }^{14} \mathrm{Be}$, ${ }^{17} \mathrm{~B}$, which possess neutron halos. $\mathrm{RNB}$ reaction studies will be important to understanding the nuclear structure of these and other equally interesting nuclei. Creation of a whole host of nuclei at high spin will lead to a better understanding of collectivity and provide access to specific orbitals with very unusual shapes such as in hyperdeformed nuclei. Regions containing the $\mathrm{N}=\mathrm{Z}$ nuclei up to ${ }^{100} \mathrm{Sn}$ or the superheavy element nuclei will finally become accessible. Unusual heavy ion reactions using for example neutron halo nuclei will be exploited leading to neutron flow phenomena, neck formation in fusion reactions, and the availability of high Q-value reactions, which due to more favorable momentum matching, will enhance certain processes and the 
populations of selected states. For proton-rich nuclei, charge-exchange reactions in heavy mirror nuclei will become possible. Another area of interest is precision tests of the electroweak standard model via parity nonconservation investigations in atomic and $\beta$-decay transitions.

Nuclear astrophysics has always had a symbiotic relation with low energy nuclear physics. At ISL there will be an explosion of new studies beginning with measurements of reaction rates for the fusion reactions occurring in older age stars. New results (e.g., masses, half-iives, and reaction rates) at the limits of stability will greatly enhance our understanding of the rp-, s-, p-, and rprocesses. In particular, such measurements will help determine the site, environment, and time scale for supernova. Atomic physics and material sciences will benefit from the wide range of new probes available that can be adjusted for half-lives, decay energies and bombarding energies. Used as dopants in materials science, high specific activity beams will permit time dependent reactions to be studied at low dopant levels thus reducing interfering collateral damage. All of these studies will in the words of the ISL report "reveal wholly new horizons for this [nuclear science] and related topics."

\section{The ISL Benchmark}

As now conceived, the ISL consists of two accelerators, a high-current $(100 \mu \mathrm{A})$, medium-energy $(0.5-1-\mathrm{GeV})$ proton accelerator followed by a low energy $(1-10-\mathrm{MeV} / \mathrm{u})$ post accelerator. In this ISOL-post accelerator approach, the high-intensity primary light-ion beam strikes a target to produce copious amounts of $\beta$ unstable nuclei which are then ionized, mass separated, and accelerated in a second accelerator. The radioactive beams are expected to be quite high in intensity (up to $10^{11} \mathrm{part} . / \mathrm{s}$ ) and be relatively free of contaminant isotopes. The figure on the next page shows the predicted intensities of radioactive beams at the ISL facility for energies between 1.5 and $10 \mathrm{MeV} / \mathrm{u}$. For lower energies $(<100 \mathrm{keV} / \mathrm{u})$, the intensities will be higher by 1 order of magnitude. (Radioactive decay losses, secondary reactions, energy losses of the primary beam, target matrix losses, and feeding from radioactive decays have not been taken into account; however, losses cased by ionization, transmission, and stripping have been included.) Projectile fragmentation, which was also seriously considered for the ISL, was deemed less suitable for the energy range of interest and would generally result in beams with higher isobaric contamination levels and considerably lower intensities $\left(10^{8} \mathrm{part} / \mathrm{s}\right.$ maximum at the projectile fragmentation beam energy with commensurate losses at lower energies). Another advantage of the ISOL technique is that other experiments requiring only the ISOL off-mass species can be performed simultaneously with the primary RNB experiment.

\section{LAMPF Involvement in ISL}

Los Alamos interest in ISL originates from the present strong exotic nuclei program which utilizes the TOFI spectrometer (see "Mass Measurements and Exotic Decay Studies Using the TOFI Spectrometer" in this report). Furthermore, we believe that the existing facilities and expertise make LAMPF the most cost effective site for ISL. In particular, LAMPF has the ideal front end accelerator. In addition, there is extensive expertise in the building of high current targets, the handling of high radiation materials, and experience in accelerator cesign and operations. There also exists a strong user support infrastructure.

To further mitigate the cost impact of ISL and maximize the scientific return, the ISL could be built at LAMPF in stages with exciting scientific opportunities made possible at each stage. With just the mass separator, a large array of nuclear science experiments could be conducted due to the expected factor of 100 increase in beam intensities over present facilities. With the first stages of acceleration $(100 \mathrm{keV} / \mathrm{u})$ a variety of materials science experiments become possible. With the first drift tube linac (1.5 MeV/u), the nuclear astrophysics program can begin measuring key reaction rates. Finally, with the entire facility one can embark on the full range of ISL experiments.

Los Alamos' involvement in the ISL is divided into three broad categories. First, we are continuing to refine the scientific case for ISL by developing selected prototype experiments. Second, we are developing a preconcept for how ISL would be sited at LAMPF. Finally, we are planning a near term R\&D program unique to LAMPF that will address several ISL technical questions. To carry out this ambitious program LAMPF has formed a radioactive beam users group. We stand ready to work with the larger North American user community in advancing the IsoSpin Laboratory initiative.

\section{PILAC}

\section{Introduction}

Exciting new areas of pion physics become possible with high-intensity, high-resolution pion beams in the energy range of $0.5-1.1 \mathrm{GeV}$. PILAC, a 
Combined RNB Intensities

(1 GeV Protons, $100 \mu \mathrm{A}$, Highest Intensities from

$\mathrm{CaO}, \mathrm{Nb}$, La, Ta, or UC targets; $1 \mathrm{~mole} / \mathrm{cm}^{2}$ )

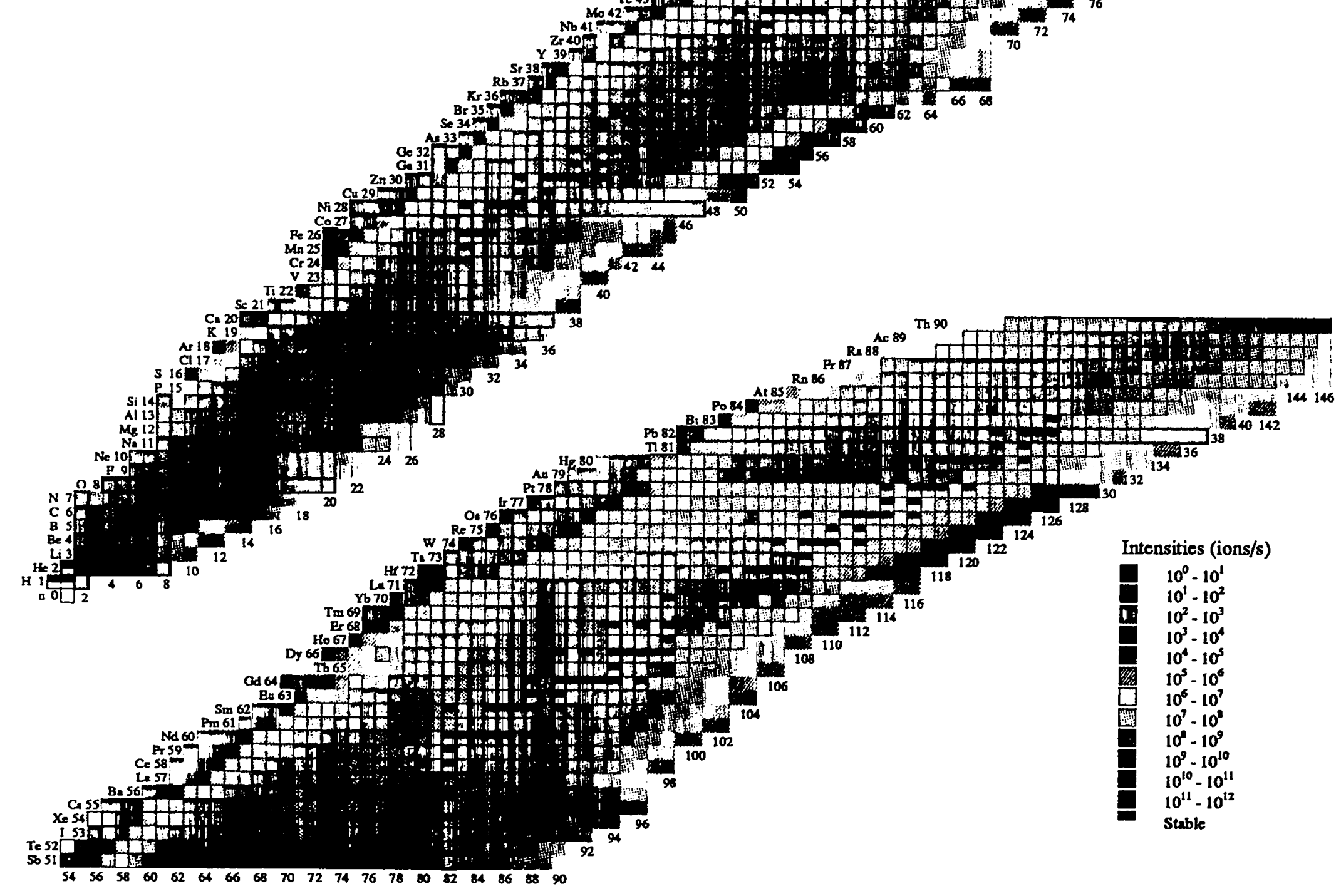


superconducting pion linear accelerator at LAMPF, would provide such new capabilities. In particular, $0.92 \mathrm{GeV}$ is optimal to produce lambda-hypernuclei through the $\left(\pi^{+}, \mathrm{K}^{+}\right)$reaction and for investigation of lambda-nucleon scattering near the $\Sigma$ threshold or cusp region. More than a dozen baryon resonances can be produced in this energy range above the $(3,3)$ resonance, as well as copious eta production through the $\pi^{-} p \rightarrow \eta n$ reaction. High-energy pion-nucleus reactions take on a new importance owing to important differences in the elementary two-body $\pi \mathrm{N}$ interaction as compared to lower energies. A very active user community has formed to provide guidance on the scientific program and performance criteria. An overview of this scientific program is given below.

In addition to the broad new physics opportunities offered by PILAC, it utilizes forefront superconducting RF technology. Recent developments in this area now make the PIAC concept a cost-effective approach to this new physics. An aggressive research and development effort is now underway at the Los Alamos National Laboratory to develop prototype accelerating cavities to demonstrate the required performance levels. The PILAC reference design, together with research and development activities, is discussed below.

\section{The Scientific Program}

Guidance as to the optimum pion energy for PILAC can be found in Fig. VI-1. Shown is the present range of energies at LAMPF, with its highest pion energies achieved in the $P^{3}$ channel but with rapidly decreasing intensity $\left(10^{6} \pi / \mathrm{sec}\right.$ at $\left.550 \mathrm{MeV}\right)$. Also indicated in Fig. VI-1 is the proposed range of energies deliverable by PILAC. A possible future upgrade to yet higher energies is also shown.

This section highlights the PILAC scientific program as developed by the PILAC Users Group. Their full report, The PILAC Users Group Report on the Physics with PILAC, contains much more detailed information and would serve as the basis of the scientific motivation for the PILAC proposal.

\section{Lambda-Hypernuclei}

The investigation of hypernuclei is driven by our desire to understand the strangeness degree of freedom in the nuclear medium: to explore the many body spectroscopy of a $\Lambda$ in the nucleus, to discover new dynamical symmetries when a nucleon is replaced by a $\Lambda$, to characterize the strong SU(3) breaking in the strange sector, to probe nuclear structure by modifying the magnetic and quadrupole moments of the nucleus in the presence of a $\Lambda$, and to explore the weak mesonic and nonmesonic decay modes of hypernuclei.

The principal production mechanisms for $\Lambda$ hypernuclei have been strangeness exchange $\left(\mathrm{K}^{-}+\mathrm{n} \rightarrow\right.$ $\left.\pi^{-}+\Lambda\right)$ and associated production $\left(\pi^{+}+n \rightarrow K^{+}+\Lambda\right)$. The $\left(\mathrm{K}^{-}, \pi^{-}\right)$at forward angles corresponds to a small momentum transfer $q$, while the $\left(\pi^{+}, K^{+}\right)$reaction is characterized by $q \geq 300 \mathrm{MeV} / \mathrm{c}$. Thus, the $\left(\mathrm{K}^{-}, \pi^{-}\right)$ process excites predominantly non-spin-flip transitions with low orbital angular momentum transfer $\Delta \mathrm{L}$, while $\left(\pi^{+}, \mathrm{K}^{+}\right)$tends to emphasize non-spin-flip transitions of maximum $\Delta \mathrm{L}$. The $\left(\gamma, \mathrm{K}^{+}\right)$photoproduction reaction is kinematically similar to $\left(\pi^{+}, K^{+}\right)$, but strongly favors spin-flip transitions. The $(\pi, \mathrm{K})$ reaction offers several important advantages. The $\pi^{+}$and $\mathrm{K}^{+}$both have long mean free paths at these energies and hence can probe more deeply bound states and collective modes of the core nucleus. Furthermore, the complementarity of the $\left(\pi^{-}, K^{9}\right)$ and $\left(\pi^{+}, K^{+}\right)$reactions permits the separation of the isospin structure of the hypernuclear states.

PILAC would provide energetic pion beams with unprecedented intensity $\left(10^{9} \pi^{+} / \mathrm{sec}\right)$ and resolution $(200 \mathrm{keV})$ for hypermuclear studies. PILAC has been

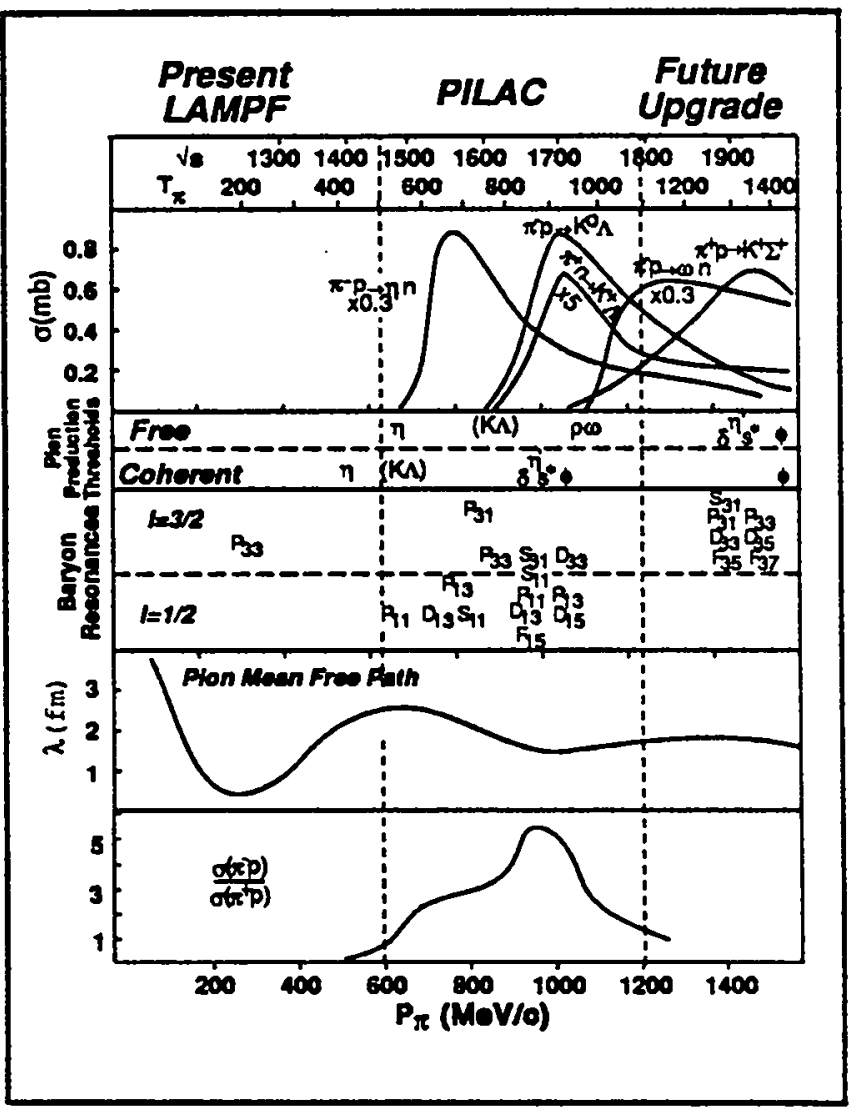

Fig. VI-1. Energy dependence of the two-body $\pi N$ interaction. 
optimized at the peak of the elementary reaction $\pi^{+} n \rightarrow$ $\mathrm{K}^{+} \Lambda$ at $1.05 \mathrm{GeV} / \mathrm{c}$. Figure VI-2 presents a calculated spectra for the the $\left(\pi^{+}, \mathrm{K}^{+}\right)$reaction using a pure particlehole interaction with no residual mixing for a mediummass target using the PILAC system resolutions as compared to what is possible today. Although the singleparticle levels are clearly seen in the low-resolution spectrum, no fine structure such as spin-orbit splitting or spin-spin effects can be seen due to the resolution. This is typical of what is measured at the present time. The high-intensity aspect of PILAC results in more than $\mathbf{3 0}$ counts per day in the weakest of the states shown in Fig. IV-2. PILAC would provide an order of magnitude improvement in resolution and two orders of magnitude more flux than what is available today.

$(\pi, \mathrm{K})$ and $(\pi, \mathrm{K} \gamma)$ experiments at PILAC will provide new information about the structure of hypernuclear spectra which is required to define the properties of the $\Lambda \mathrm{N}$ interaction - the spin-spin, $\Lambda$ spin-orbit, induced nucleon spin-orbit, tensor, and central terms. The $3-\mathrm{MeV}$ resolution available in present $(K, \pi)$ and $(\pi, K)$ experiments is inadequate to constrain the theoretical models to the degree required to define these two-body terms, to see dispersive effects due to $\Lambda \mathrm{N}-\mathrm{EN}$ coupling, or to understand the size or spin dependence of $\Lambda \mathrm{NN}$ threebody forces. Resolution of the order of $200 \mathrm{keV}$ is required to adequately define the level structure.
Additionally, at nonzero angles the $(\pi, K)$ reaction produces polarized hypernuclei, negligible for unnatural parity states, and substantial for natural parity states. This new information offers a promising spectroscopic tool with which to improve the definition of the $\Lambda N$ interaction.

Polarized lambdas can be produced in a liquidhydrogen target, tagged by the $K_{s}$ decay, and allowed to rescatter in the same liquid-hydrogen target. Using this technique, it is possible to make not only cross-section measurements of lambda-proton scattering near threshold, but also spin-transfer measurements, since the lambda is self analyzing. PILAC would provide the necessary beam energy, intensity, and beam quality to make not only a precise determination of $\sigma$ and $A_{y}$, but also the spin transfer observables $D_{\mathrm{NN}}, D_{S S}$, and $D_{S L}$ to an accuracy of \pm 0.05 in 100 angle-momentum bins within a month's running time. Most of the existing data is of total cross sections, derived from a few hundred bubble chamber events. This would therefore represent a major contribution to the determination and understanding of the hyperon-nucleon interaction for which many models and predictions exist but remain untested.

The nonmesonic weak decay of $\Lambda$-hypernuclei provides our highest momentum transfer (shortest range) probe of the $\Lambda \mathrm{N}$ interaction. The production of ${ }_{\Lambda}^{4} \mathrm{H}$ and ${ }_{\Lambda}^{4} \mathrm{He}$ and study of their subsequent weak decays

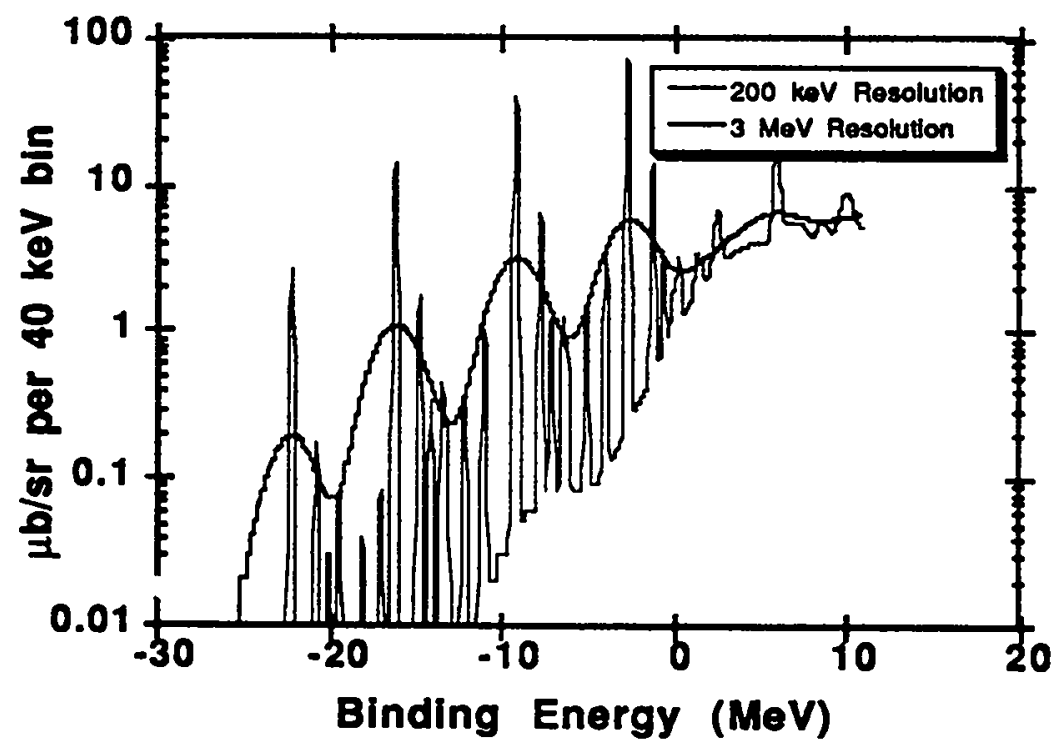

Fig. VI-2. Calculated $\left(\pi^{+}, K^{+}\right)$spectrum for medium-mass target with the PILAC resolution of 200 keV compared with the 3-MeV resolution available at present. 
using the high statistics capability of PILAC provides our best means of exploring the detailed nature of both $\Lambda+$ $N \rightarrow N+N$ and $\Lambda \rightarrow N+\pi$ decays in the same nucleus. (Pauli blocking quickly suppresses the mesonic decay mode as $A$ increases.) In particular, precision measurements of neutron-stimulated decay rates $\Lambda+n \rightarrow$ $n+n$ could provide a definitive test of nonmesonic decay calculations, helping to answer such questions as whether the $\Delta I=1 / 2$ rule, which holds for hyperon and kaon decay, also applies to the $\Lambda+N \rightarrow N+N$ process.

\section{Baryon Resonances}

The study of the baryon resonances occupies a unique place in the field of hadronic physics. It provides our most direct link between this description of the nucleus (and hadronic reactions) in terms of nucleons and mesons with the underlying fundamental theories. Theories take on various forms generally based on (or inspired by) QCD.

As seen in Fig. VI-1, the PILAC energy range also includes more than a dozen baryon resonances $\left(\mathrm{N}^{*} \mathrm{~s}\right.$ and $\Delta s$ ) above the $(3,3)$ resonance. Many of these resonances are poorly characterized, as little data or data of poor quality presently dominate this energy range. As noted by the Particle Data Group, virtually all information on baryon resonance masses, widths, and elasticities comes from pion-nucleon data. Inasmuch as the resonances above the $\mathrm{P}_{33}$ are highly overlapping, the parameters for these states would be obtained by partial-wave analysis of extensive PILAC scattering data over this energy range. Some of the states have unique signatures, such as the $S_{11}$, which decays a large fraction of the time into final states consisting of two $\eta$ 's. The information derived from the pion scattering will be highly complementary to the CEBAF program, which proposes to measure form factors for many of these resonances. PILAC would provide the necessary pion beam energy, purity, and quality for the precision data needed to clearly define this region of resonances.

\section{High-Energy Pion-Nucleus Reactions}

The little work that has been done in this area at LAMPF and BNL in the high-energy region has been with low-intensity low-resolution beams. At energies above $300 \mathrm{MeV}$, the qualitatively different properties of the elementary pion-nucleon interaction and the much shorter wavelength of the pion alter the character and type of physics questions that can be addressed. The energy and momentum transfers available for nuclear studies with pions at PILAC complements the work with electrons at
CEBAF, with the pion adding the isospin degree of freedom to the reaction as well as unique properties of the pion such as double charge exchange.

At high energies, the much weaker two-body pionnucleon interaction results in a much longer mean-free path in nuclei as compared to resonance energies, second only to the $\mathrm{K}^{+}$in this energy range. At $1 \mathrm{GeV}$ the pion wavelength is about one Fermi, about the size of a single nucleon, as compared to more than four Fermis at 180 $\mathrm{MeV}$. These aspects of high-energy pions provide sensitivity to the details of the spatial dependence of the ground-state and transition densities, as well as sensitivity to the modifications of the nucleon properties in the nuclear medium. To fully exploit the short wavelength of high-energy pions, measurements need to be carried out to substantial momentum transfers. Since the cross sections are quite small at large momentum transfer, the high intensity provided by PILAC will be essential.

Pion double charge exchange (DCX) is unique in that it is the only reaction of a noncomposite particle that requires the participation of two nucleons in all of its manifestations. DCX will clearly benefit from the shorter wavelength of the higher energy pions. With the shellmodel correlation sensitivity of low-energy DCX already clearly demonstrated, the higher energies at PILAC will allow for a detailed study of the short-range behavior of nucleons in the nucleus. The distance scale being probed at PILAC may not be adequately described in terms of only nucleon degrees of freedom, and the transition to mesonic or other sub-nucleonic degrees of freedom could be investigated. Recent calculations indicate that an even more dramatic reduction in the DCX cross section over what is seen at $50 \mathrm{MeV}$ occurs at these higher energies. Sensitivity to more exotic reaction mechanisms will therefore be enhanced.

The isospin dependence of the two-body interaction is also quite different from lower energies. As seen in Fig. VI-1, the ratio of $\pi^{+} p$ to $\pi^{+} n$ cross sections on resonance is reversed near $1 \mathrm{GeV}$. This feature can be exploited to test our understanding of the isospin dependence of the pion-nucleus interaction and the isotopic composition of various nuclear transitions.

The increased energy of pions opens the possibility of a broad program in quasi-elastic scattering. At $1 \mathrm{GeV}$, the momentum transfer to the nucleon exceeds the Fermi momentum for angles greater than $25^{\circ}$. Under these conditions, the pion will scatter incoherently from the individual nucleons. This reaction provides an independent and complementary probe of $p_{2}$ on dynamics in the nucleus and of that of the nucleon in the nuclear medium, analogous to nucleon and electron scattering. The possibility of "tagging" the $S_{11}$ resonance in the 
nuclear medium through its decay into final states with $\eta$ 's becomes possible. This would provide a method of investigating the interaction of this highly-excited hadron with the nuclear medium.

The decreasing two-body interaction strength and smooth energy dependence also has important implications for theoretical analysis and interpretation. Perturbation theory becomes increasingly more convergent, making theoretical analysis simpler and more reliable. The relative size of unconventional mechanisms, such as meson exchange currents, to the conventional pion-nucleon interaction is therefore enhanced.

\section{Meson Decays}

Of considerable interest is the beta decay of the pion. The rate of this decay, $\pi^{+} \Rightarrow \pi^{0} \mathrm{e}^{+} v_{\mathrm{e}}$, is proportional to the $V_{u d}$ element of the CKM matrix. A precision measurement would provide a test of the standard model, which predicts this rate to $0.1 \%$, as well as providing a check of the unitarity of the CKM matrix. Pion beta decay offers an alternative approach to this information as compared with nuclear beta decay, which now determines these quantities. The corrections in the two processes are quite different and, in the case of pion beta decay, appear to be more manageable. This experiment was performed at LAMPF in 1985 with a $3.8 \%$ determination of the rate. PILAC would provide the necessary energy, intensity, and beam quality to push the measurement to $0.2 \%$.

A dominant channel in the total $\pi^{-} p$ total cross section near $750 \mathrm{MeV}$ is $\eta$-production. Almost $7 \%$ of the total cross section goes into this channel. As such, PILAC would be able to provide high fluxes of tagged or untagged $\eta$ 's for rare and "ordinary" decay studies. The expected yield on a $30-\mathrm{cm}$ liquid hydrogen target is $10^{10} \eta /$ day. Searches for a number of very interesting rare and forbidden decays probing physics outside the standard model would become possible. These include tests of $\mathrm{CP}$ violation in the muon polarization from $\eta \rightarrow$ $\mu^{+} \mu^{-}$, certain classes of pseudoscalar interactions in $\eta \rightarrow$ $\mathrm{e}^{+} \mathrm{e}^{-}$and lepton family number violations in $\eta \rightarrow \mu^{+} e^{-}$. A number of "ordinary" decays of the $\eta-2 \pi, 2 \pi \psi, \pi \gamma \gamma$ - provide important probes of the ways in which the chiral symmetry of QCD is manifested. PILAC would npen this area of research to precision study.

\section{The Facility}

A brief overview of the reference design for PILAC will be given. The major subsystems that define performance and have the greatest impact on the scientific program will be described. The overall site plan is shown in Fig. VI-3.

\section{Pion Production and Injection}

A new zero-degree target cell will be constructed in Area $B$ at LAMPF, displacing the present Nucleon Physics Laboratory. The zero-degree arrangement offers the advantages of higher pion fluxes and increased source brightness. A much thicker production target can be used while still maintaining the small source spot size required by PILAC. The $1-\mathrm{mA} \mathrm{H}^{+}$primary beam would be directed to this new target cell, with the present Area A and PSR programs sharing a 1-mA H- beam. Based on pion production rates and survival fraction considerations, the optimum injection energy is $365 \mathrm{MeV}$. A new injection line has been designed to accept a large-phasespace pion beam ( $230 \pi$-mm-mrad) and transport it to the linac without introducing large chromatic aberrations as is common with existing pion beam lines.

\section{The Superconducting Pion Linac}

A unique feature of pion acceleration is the fact that the beam decays during acceleration. It is therefore critical to perform the acceleration as quickly as possible, while meeting all conditions on beam dynamics. Central to this point is the issue of accelerating gradients and survival fractions. Figure IV -4 indicates the calculated $\pi^{+}$flux per milliampere of primary current for PILAC as a function of accelerating gradient. As can be seen in this figure, the design goal of $10^{9} \pi^{+} / \mathrm{sec}$ is achieved at an accelerating gradient of $12.5 \mathrm{MeV} / \mathrm{m}$. Also shown in Fig. VI-4 are the expected yields for the proposed KAON facility under the assumption that the output phase space of the highresolution beam line is the same in both cases. The yield for the AGS and AGS plus Booster is also shown under similar assumptions.

A more detailed discussion is given on the research and development activities now in progress to meet the superconducting cavity requirements of PILAC is given below.

As currently envisioned, the linac would be constructed of seven-cell $805-\mathrm{MHz}$ superconducting niobium cavities. There would be 45 of these cavities forming the $100-\mathrm{m} 560-\mathrm{MeV}$ linac. The cavities would be contained in liquid helium cryostats operating at $2^{\circ} \mathrm{K}$ with cavity gradients of $25 \mathrm{MV} / \mathrm{m}$, accelerating gradients of $12.5 \mathrm{MeV} / \mathrm{m}$, and a $\mathrm{Q}$ of $5 \cdot 10^{9}$. The beam loading at PILAC is negligible. As such the power requirements are given by the cavities themselves. Power consumption is estimated at 100 watts per cavity, or $4.5 \mathrm{~kW}$ total. 


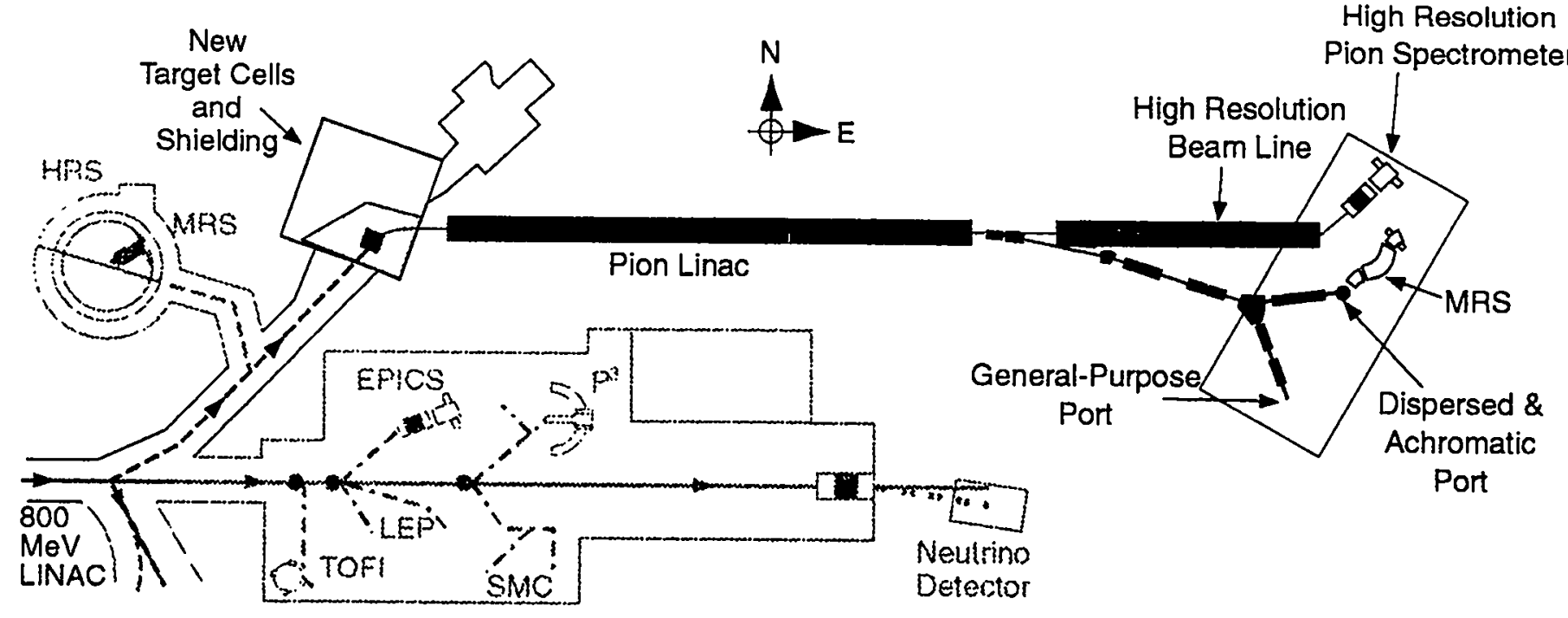

Fig. 3. Location of PILAC and experimental area at $L A M P F$

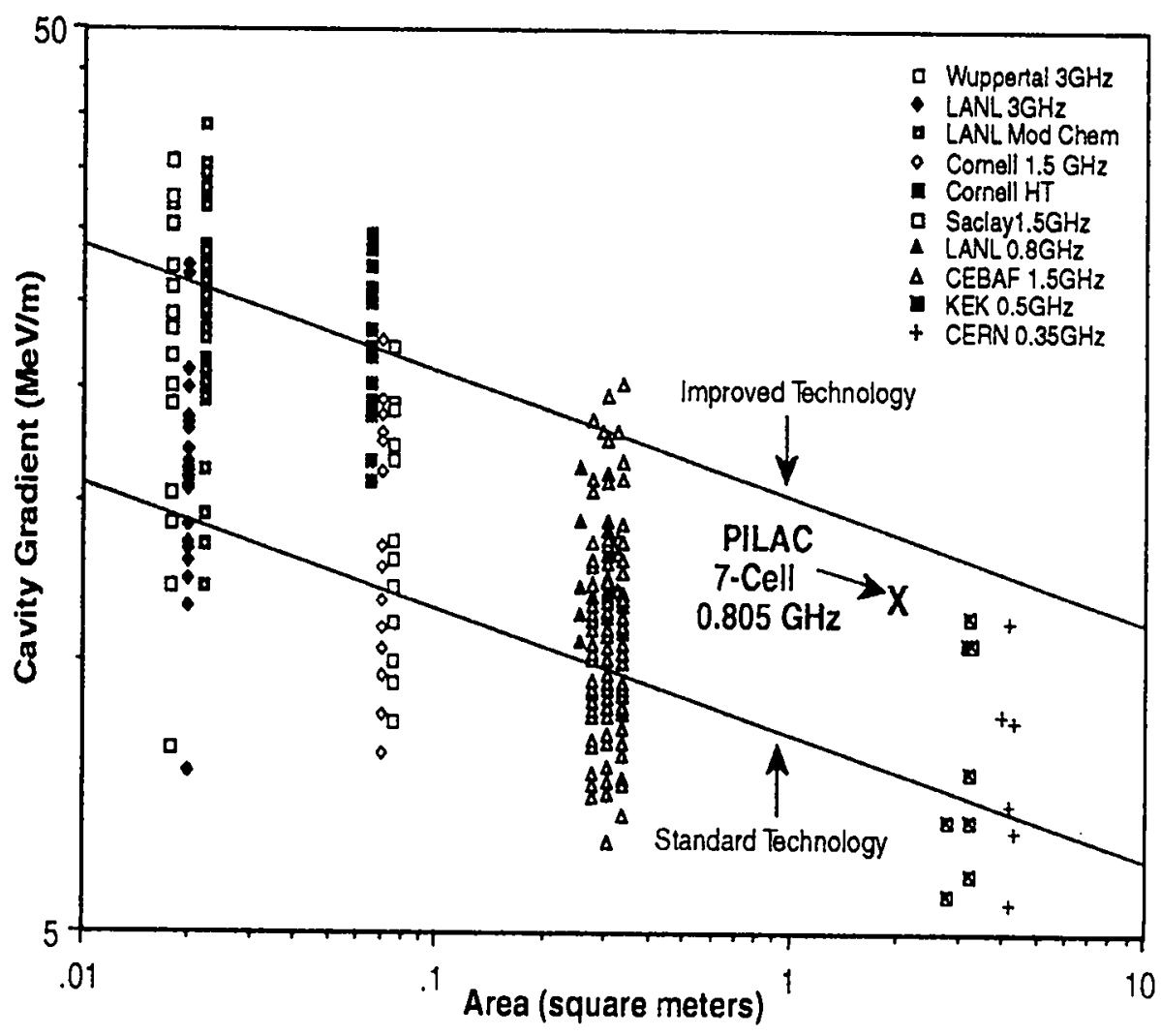

Fip. 5. Summary of cavity gratient results achieved at various laboratories. The characteristics of the canities needed for PILAC are shoun. 


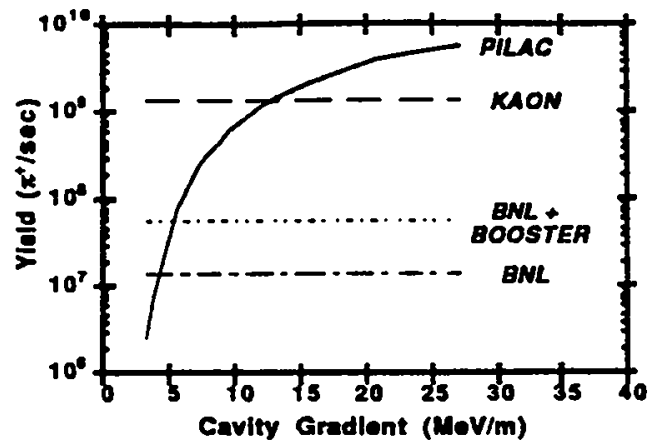

Fig. VI-4. Calculated $\pi^{+}$yield for PILAC and other facilities.

All cavities are independently phased. This allows a broader range of tuning parameters that take ad vantage of individual cavity performance levels. Extensive beamdynamics studies have been conducted. During the early stages of acceleration, the RF is phased so as to perform a longitudinal phase rotation to provide an output beam minimized in momentum width. This is optimal for injection into the high-resolution beam line.

\section{Beam Lines and Experimental Facilities}

Two beam lines are envisioned for PILAC, a highresolution channel that feeds a high-resolution spectrometer system for hypernuclear and pion-nucleus spectroscopy and a general-purpose beam line for lower resolution and large acceptance detector systems. The general-purpose beam line could also serve as an injection line for a possible future upgrade to yet higher energies. Several beam-sharing schemes are being considered.

The high-resolution system is designed for $200-\mathrm{keV}$ resolution, driven primarily by the requirements of the $\left(\pi^{+}, \mathrm{K}^{+}\right)$hypemuclear spectroscopy program. The channel utilizes vertical bends with a momentum dispersion of $25 \mathrm{~cm} / \%$. The momentum resolution has been calculated with ion-optic programs up to fourth order. While a new large-acceptance spectrometer is highly desirable and ultimately needed, the existing EPICS spectrometer might satisfy the early round of experiments with some compromise in the detected kaon rate due to its smaller acceptance. In the $\left(\pi^{+}, \mathrm{K}^{+}\right)$reaction, the outgoing kaon has a momentum of about $720 \mathrm{MeV} / \mathrm{c}$ for an incoming 1.05-GeV/c pion momentum. The present EPICS spectrometer can easily accommodate this momentum at the required resolution.

The present Medium Resolution Spectrometer (MRS) at LAMPF could serve as the lower resolution (2-3 MeV) spectrometer on the general-purpose channel. This would provide sufficient resolution for some pion scattering and DCX experiments up to full energy. Large acceptance detectors based on existing equipment such as the MEGA solenoid and large dipole magnets are also being considered for this channel for light meson decay studies and lambda-proton scattering experiments.

\section{Research and Development}

Research and development (R\&D) is underway within the Medium Energy Physics (MP) and Accelerator Technology (AT) Divisions at LANL, supported by Laboratory discretionary funds. These funds were $\$ 2.6 \mathrm{M}$ in FY91, with $\$ 3.1 \mathrm{M}$ in FY92 and about $\$ 2.5 \mathrm{M}$ anticipated in FY93. During FY91 the effort centered around systems analysis, such as beam transport and dynamics studies, and superconducting-cavity development. A reference design now exists for the overall system as described in the previous sections.

In FY92 the R\&D effort concentrates on cavity development, given that high-gradient, high- $Q$ superconducting cavities are central to the pion accelerator concept. The goal is to demonstrate that the necessary gradient $(>12 \mathrm{MV} / \mathrm{m})$ and $Q\left(>2 \times 10^{9}\right)$ can be achieved in low-frequency $(805 \mathrm{MHz}$ ) multi-cell cavities required for the pion linac, and that both the gradient and $Q$ can be preserved in the accelerator environment.

With conventional technologies, accelerating gradients of 5-7 MV/m can be achieved under accelerator conditions. Theoretical limits for the accelerating field for niobium are as high as $50 \mathrm{MV} / \mathrm{m}$, set by the critical magnetic field. Several important limitations have prevented realization of these high fields. Although significant progress has been made in cavity performance over the past several years by overcoming limitations associated with multipacting and thermal breakdown, present performance is limited by field emission.

Figure VI-5 presents a summary of recent results worldwide on superconducting spherical cavities. Each symbol on the plot represents one result of a test for maximum gradient available. The results for cavity gradient observed are plotted as a function of surface area of the cavity tested. Clearly, there is a correlation observed, with the result being that it is more difficult to obtain a high gradient in a large cavity (e.g., low frequency or many cells). The Los Alamos results at $3 \mathrm{GHz}$ are divided into two groups, one using standard chemistry processing, the second using a new chemical processing treatment recently developed at LANL. As shown in the figure, the mean gradient achieved in the new chemical processing tests is nearly $50 \%$ greater than 
that achieved with the standard chemistry. The Comell data are also divided into two groups, one using standard techniques, and the second using a heat treatment. The heat treated cavities achieved a gradient higher than the standard cavities. The average result of the heat treatment is approximately $50 \%$ higher than that obtained with standard treatment.

It is clear from Fig. VI-5 that the Los Alamos results to date are comparable to the best achieved at other laboratories. Our $3-\mathrm{GHz}$ results equal or exceed those obtained at worldwide, and the results of the small number of 0.8-GHz single-cell tested at LANL to date (all using standard chemistry) are comparable to the results obtained at CEBAF.

The two methods of gradient improvement, namely heat-treatment and improved chemistry, suggest that the necessary improvement in cavity gradient up to $12.5 \mathrm{MeV} / \mathrm{m}$ can be obtained by either technique. A major milestone for the R\&D plan is a demonstration of improvements realized by these improved processing techniques for larger cavities.

\section{Cost and Schedule}

Table VI- 1 has summary costing information derived from detailed cost estimates, including reasonable engineering, design, inspection, and contingency values. The major items such as accelerating structures, cryogenics and experimental equipment are based on recent CEBAF values. The current $R \& D$ plan calls for demonstration of cavity performance in early 1993. If successful, this would allow for an FY95 construction start, with funding needed over three years. The "Base" option includes the reconfiguration of Area B, a new target cell, shielding, new injection line, linac, high-resolution beam line, and use of the existing EPICS spectrometer. "Option $1^{\text {" }}$ is the incremental cost to add beam sharing. two additional general-purpose beam lines, and the existing MRS spectrometer. "Option 2" is the incremental cost of the new high-resolution spectrometer.

\section{Summary}

PILAC is being designed to provide beams of $10^{9}$ pions per second with an overall system resolution of $200 \mathrm{keV}$ at energies up to $1.1 \mathrm{GeV}$. A reference design including all key components has been completed which meet these requirements. An aggressive $R \& D$ program is now underway to demonstrate the required superconducting cavity performance for the linac in order to achieve the desired goals. PILAC will offer two orders of magnitude more flux and an ordes of magnitude better resolution than other 1-GeV pion beams available today. Additionally, the PILAC beam will be of extraordinary good quality and purity due to the acceleration process. A broad range of scientific studies become possible with PILAC of interest to many areas of nuclear physics. In particular, the upper end of the PILAC energy range is optimal for production of lambda hypernuclei through the $\left(\pi^{+}, \mathrm{K}^{+}\right)$reaction. More than a dozen baryon resonances are accessible with PILAC. Significant differences in the elementary twobody $\pi \mathrm{N}$ interaction at high energies will provide qualitatively new areas of investigation in high-energy pion-nucleus reactions. Copious production of pions and eta mesons will allow for precision studies of their decays. PILAC offers a cost-effective approach to a dedicated facility for forefront nuclear physics research in the U.S.

Table VI-1

PILAC Costing Summary (SK)

Totals

1. Beam Transport

2. Accelerator Systems

3. Conventional Facilities

4. Cryogenic Systems

5. Experimental Equipment
Base

19,860

12,577

8,553

9,700

100
Option 1

Option 2

4,850

1,525

100

10,200

$\begin{array}{lc}\text { Sub-total } & 50,790 \\ \text { ED\&I @ 15\% } & 7,619 \\ \text { Installed cost } & 58,409 \\ \text { Contingency } & 17,392(30 \%) \\ \text { Total Estimated Cost } & \mathbf{7 5 , 8 0 1}\end{array}$

6,475

957

7,432

10,200

1,530

1,863 (25\%)

11,730

9,295

2,933 (25\%)

14,663 


\section{Pulsed Lepton Source (PLS)}

The pulsed lepton source (PLS) is shown in plan view in the figure on the next page. $\mathrm{H}^{-}$beam from LAMPF is accelerated to $800 \mathrm{MeV}$ and deflected into an injection line to the Proton Storage Ring (PSR). The beam is injected by stripping in an external B field followed by stripping the second electron while passing the beam through a thin foil inside the ring. Beam is accumulated for a substantial fraction of a millisecond until $3 \times 10^{13}$ protons are accumulated. Extraction then occurs in a single turn so that a very high intensity burst of protons is produced for about $0.25 \mu \mathrm{s}$. At present this beam is used at 20-Hz repetition rate at the Los Alamos Neutron Scattering Center (LANSCE) for studies in nuclear physics and material science. At this repetition rate, $80 \mu \mathrm{A}$ is available routinely, while $100 \mu \mathrm{A}$ has been demonstrated fur short periods. The PLS would use a further $40 \mathrm{~Hz}$ for fundamental muon physics, material science with muons, and neutrino physics.

Limitations on the delivered flux exist because an instability occurs in the ring when accumulated protons exceed about $3.5 \times 10^{13}$, and because of beam loss activating the ring itself making hands-on maintenance difficult. The PLS idea avoids the first limitation by limiting accumulated protons and increasing the total repetition rate to $60 \mathrm{~Hz}$. Beam losses are reduced by increasing the brightness of the $\mathrm{H}^{-}$beam, going to direct injection and increasing the aperture of the extraction system. Each of these is believed to be a straightforward application of known accelerator techniques and engineering, and the accelerator staff believe that the achievement of $300 \mu \mathrm{A}$ of delivered current is feasible.

The physics program is discussed in three sections, fundamental muon physics, neutrino physics, and materials science with muons.

\section{Fundamental Muon Physics}

Fundamental physics with muons has been addressed in several documents over the last decade. These documents present a strong case for a rich program of physics opportunities with muons, requiring $\mathrm{CW}$ and pulsed sources. Even though there have been considerable advances in experimental technir.es during this period, many topics retain their position on the list of subjects of interest because of the lack of adequate facilities. For the most part, these experiments await the advantages to be provided by beams of pulsed muons, including freedom from short-lived backgrounds, line-width narrowing, a precise zero in time, and higher efficiency deriving from coincidence with laser pulses.
The following short discussion of topics in fundamental muon physics provides examples of the advantages of a pulsed source of muons. Leading the list is a more precise measurement of the muon lifetime, for which the advantage of a pulsed source of muons centers on background-free decays at long lifetime. The present level of precision for the muon lifetime was obtained from experiments at Saclay and TRIUMF using about $10^{9}$ muon decays. These resulted in the determination of the value of the weak decay constant, $G_{\mu}$, to a precision of $17 \mathrm{ppm}$. An experiment with an intense source of muons from a pulsed source with a sharp to and a tackground-free environment should allow at least an order-of-magnitude improvement in this fundamental parameter of the Standard Model.

Pulsed muons would provide an ideal source for experiments using the line-narrowing technique of old muonium for an improved measurement of the hyperfine interval, $\Delta v$, and $\mu_{\mu} / \mu_{\mathrm{p}}$. This experiment would follow one currently planned for LAMPF that aims to determine $\Delta v$ to $5 \mathrm{ppb}$ and $\mu_{\mu} / \mu_{\mathrm{p}}$ to $50 \mathrm{ppb}$. Further improvement in the precision of these quantities will allow a critical test of QED and provide input to the determination of the muon $\mathrm{g}-2$ value.

An intense pulsed muon beam is essential to exploit recent developments in laser technology to study the spectroscopy of exotic atoms. New experiments will include the $15-2 S$ transition in muonium, the fine structure and Lamb shift in $\mu^{-3} \mathrm{He}$ and $\mu^{-4} \mathrm{He}$, and hyperfine structure and Lamb shift in $\mu^{-} p$. These measurements will yield accurate values for the nuclear radii of these light nuclei, will study the behavior of the muon as a heavy electron, and will provide further sensitive tests of QED.

Additionally, a pulsed muon facility will foster a new generation of experiments in muon capture. New techniques for producing polarized muonic atoms that have been developed at LAMPF can be exploited at a pulsed muon facility. This will allow improved determinations of the weak pseudoscalar coupling, tests of second-class currents, and probes of $\mathrm{T}$ violation. Via radiative muon capture, collective nuclear excitations may be studied, and possible modifications of the weak current in a nuclear medium may be investigated.

\section{Neutrino Physics}

The reason that PLS extends experimental capabilities in neutrino physics derives from two conditions. The first is that, because of the duty factor $\left(10^{-5}\right)$, background from cosmic rays is virtually eliminated, so that elaborate veto shields are unnecessary. This allows economical 


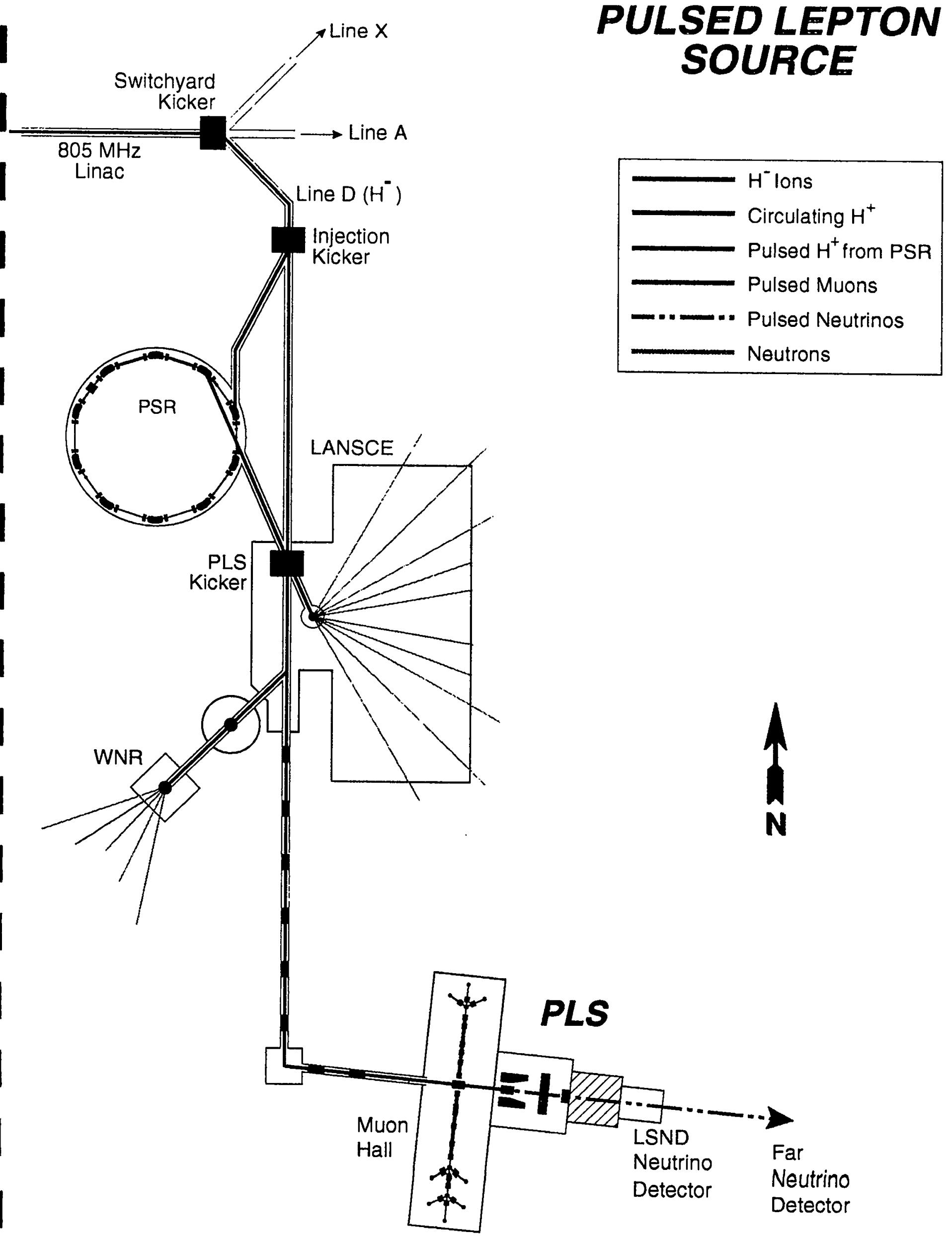


construction of larger detectors on the surface. PLSND is an example of such a detector, with 2000 tons of active mass, following the design of LSND in detail. The second is that the pulsed nature of the proton beam allows the time structure of $\pi$ and $\mu$ decays to be used to separate $v_{\mu}$ from $v_{e}$ in an especially clean manner.

Neutrino-proton elastic scattering at low $Q^{2}$ has emerged as an important measurement in the physics of the nucleon. At low $Q^{2}$ the measurement is of an appropriate charge, in the case of neutrino scattering from the proton the cross section is dominated by the axial contribution. In this neutral current process, the contribution to the cross section from $u$ and $d$ quarks is identical to that measured in neutron decay, so that any difference from this value may be attributed to a contribution to the nucleon spin by strange quarks. Deep inelastic lepton scattering also bears on this problem, with emphasis on the nucleon structure. Unlike neutrino scattering, in order to derive an absolute value for the contribution it is necessary to assume SU(3) symmetry. It is our view that $G_{s}$ is sufficiently central to the understanding of $Q C D$ in the nucleon that a program of experiments will be necessary to push precision as far as possible. PLS offers high rate and freedom from cosmic ray background giving ultimate sensitivity.

At present, fundamental neutrino physics is in a very exciting state. Studies of the solar neutrino problem have given hints that neutrino oscillations occur, and the measurements of $v_{\mu}$ flux in detectors sensitive to atmospheric neutrinos also give hints that there is a deviation from conventional expectations. At the same time, measurements of $\sin ^{2} \theta w$ at CERN, together with the measurement of $\sin ^{2} \theta w$ from weak-electromagnetic interference in atomic physics, are putting severe constraints on the existence of particles other than those required in the Standard Model. In discussing a neutrino program at PLS, it is necessary to consider that any of these possibilities may become firm, and further experimentation will be necessary to extend our knowledge.

We discuss the opportunities in fundamental neutrino physics at PLS under the following two scenarios:

(1) The oscillation solution for the solar neutrino problem is generally accepted. A likely value for the mass of $v_{\tau}$ is in the range 0 ' to $1 \mathrm{eV}$. Mixing between $v_{\mu}$ and $v_{\tau}$ is large, as required by the atmospheric neutrino data.

If the oscillation probability for $v_{\mu} \rightarrow v_{\tau}$ is substantial, and the mass-difference-squared is in the range $0.01<\Delta \mathrm{m}^{2}<1 \mathrm{eV}^{2}$, then a long-base-line experiment at PLS is very attractive. $v_{\tau}$ appearance experiments require neutrino energies in excess of $5 \mathrm{GeV}$ in order to produce $\tau$ leptons. The distances necessary for significant oscillation in this mass range make it impossible to build large enough detectors that are sensitive to $\tau$ leptons. If it is not economically feasible to detect $v_{\tau}$ by appearance methods, then $v_{\mu}$ disappearance is the only way to detect this oscillation. At PLS energies, systematic errors that have made such measurements difficult in the past can be mitigated by measuring the event ratio

$$
N\left(v_{\mu}+{ }^{12} C \rightarrow \mu^{-}+{ }^{12} N\right) / N(v+p \rightarrow v+p) .
$$

Because flavor oscillations leave the denominator unchanged, this ratio is insensitive to flux uncertainties, while being sensitive to oscillations. This measurement would be made with LSND in the near position and PLSND in the far position, $500 \mathrm{~m}$ from the source. In three years of running, for large $\Delta \mathrm{m}^{2}$, the limit on mixing (at $90 \%$ confidence) is 0.05 ; for full mixing, the lower limit on $\Delta \mathrm{m}^{2}$ is $5 \times 10^{-3} \mathrm{eV}^{2}$.

(2) The top quark mass is known, and the value for $\sin ^{2} \theta_{W}$ derived from measurements at LEP are not in agreement with that from atomic physics measurements.

Radiative corrections to the measurements at the $\mathrm{Z}^{0}$ energy depend not only on the top quark mass but also on any additional particles that are postulated to augment standard electroweak theory. If measurements of weakelectromagnetic interference disagree with measurements at LEP, then measurements of the event ratio of neutral to charged currents, such as appears in

$$
R=\frac{\sigma\left(v_{\mu} e\right)}{\sigma\left(v_{e} e\right)+\sigma\left(\bar{v}_{\mu} e\right)},
$$

for neutrino-electron elastic scattering at PLS, will be necessary to resolve the direction of new physics. By measuring this ratio, a precision of better than \pm 0.002 in $\sin ^{2} \theta_{W}$ is attainable in three years of running with PLSND placed in the near position.

Neutrino physics at PLS probably requires the reuse of LSND at first and, perhaps, for a substantial period thereafter. The same technology looks favorable for a substantially larger detector, making possible precision measurements that are unique to this facility. If both scenarios (1) and (2) above evoive, we shall be faced with the enviable dilemma of whether to locate PLSND in the far position for (1) or in the near position for (2).

\section{Materials Science with Pulsed Muons}

Positive muon spin rotation, relaxation, resonance $(\mu+S R)$ has become a powerful tool for materials science, 
with applications to a growing range of problems in physics and chemistry. The PLS, with multiple muon target stations, will make this tool very much more accessible to users in the U.S. and throughout the world.

The positive muon has some notable advantages over other more conventional probes (nuclear magnetic resonance, neutron scattering, Mossbauer spectroscopy, etc.). For example, it is extremely sensitive to small magnetic moments $\left(<10^{-3} \mu_{B}\right)$, is localized at an interstitial rather than substitutional lattice position as in NMR, and can detect correlation times in the interval $10^{-5}-10^{-12} \mathrm{~s}$. It is an infinitely dilute probe with minimal lattice disturbance. It is also a hydrogen isotope that can be used to study molecular systems. Variations of the mSR technique are now appearing, each one offering new ways to investigate materials. The proposed PLS not only will allow the range of currently known techniques to be applied, but, with eight muon target stations, will provide ample time for both experiments and the development of new techniques.

A variety of materials science problems will be addressed at the PLS using these muon target stations. For example, magnetically ordered systems are very amenable to $\mu S R$ because of the sensitivity of the muon magnetic moment to local fields. The determination of the onset of magnetic ordering in the heavy-fermion superconductor $\mathrm{UP}_{3}$, the magnetic phase diagram for the high-temperature superconductor $\mathrm{Y}_{1-x} \operatorname{Pr}_{x} \mathrm{Ba}_{2} \mathrm{Cu}_{3} \mathrm{O}_{x}$, and local moment formation in spin glasses are examples of recent advances made by $\mu S R$. In metallic lattices, the muon mimics the proton and, consequently, allows the study of localization and diffusion common to hydrogen in metals. Recently, studies in $\mathrm{Cu}$ have provided valuable information on the crystallographic site, electronic structure, and low-temperaure mobility of the interstitial defect. Of current tochnological interest is the problem of hydrogen in semiconductors. Muonium in a semiconductor is expected to behave much like hydrogen and thus can be studied as the analog case. For example, if muonium is trapped at impurities, viz., acceptors, it behaves analogously to hydrogen passivation of dopants. Experiments involving impurities in $\mathrm{Si}, \mathrm{Ge}$, and $\mathrm{GaAs}$ are now being conducted using various $\mu S R$ techniques. Zero-point motion, isotope effects, and hyperfine coupling constants have been investigated by substituting muonium for hydrogen in solids, liquids, and gases. Much of the current understanding of the vortex state in high-temperature superconductors, especially measurement of the London penetration depth, has been provided by HSR experiments.

These are only a few examples of the many areas of materials science that can benefit from $\mu S R$ studies. The unique features of the muon as a probe, coupled with the advantages of the proposed facility, would strengthen the position of LANL, and the U.S., in materials research.

A project definition study has been completed for PLS which establishes the scope of the facility together with a cost estimate which is believed to be as accurate as can be achieved without a complete engineering design. The budget numbers are shown in Table VI-2. The physics program looks to us to be attractive, with many of the advantages that come with the unique character of the facility. Much of this physics can not be done elsewhere and the exploitation of this opportunity seems to us to be intensely desirable.

\section{Ultracold Neutrons}

Ultracold neutrons (UCN) provide unique opportunities to study fundamental symmetries and the structure of the weak interactions. Due to the extremely long wavelengths of UCN $(\sim 1000 \AA)$ and low kinematic energies $\left(-10^{-7} \mathrm{eV}\right)$, it is possible to totally confine UCN within bottles for periods of several hundred seconds, allowing detailed studies of neutron properties and fundamental symmetries. The long wavelengths also provide unprecedented sensitivity to study a variety of quantum mechanical effects. And since the kinetic energy of $\mathrm{UCN}$ is comparable to the gravitational potential energy, detailed studies of gravitational effects become possible.

The long-range plan formulated by NSAC in 1989 stated that "A facility judged to be of major importance to this field (precision tests of fundamental interactions) is a source of cold and ultracold neutrons. The lack of firstrate sources in the U.S. is limiting basic experiments on the parity violation, time-reversal violation, and the lifetime, electric dipole moment, and beta-decay angular correlations of the free neutron. High flux cold neutron sources ... allow significant improvements in tests of fundamental symmetries, but it has been shown that ultracold neutrons are superior in virtually every aspect." Unfortunately, there is still no source of UCN in this country. The only two sources of UCN available have been at the Institute Laue-Langevin (ILL) in Grenoble and the Kurchatov reactor in Leningrad. However, both of these reactors are presently shut down. The demand for use of UCN exceeded the availability, even when both reactors were running. It now appears quite feasible to construct an UCN source at LAMPF, which would provide an intense source of UCN in the near futue at rather minimal cost to meet this need.

The production of UCN relies on a source of cold neutrons and a converter that can shift the cold neutrons 
Item

Cost in MS

Eacility

Improvements to the PSR

Beam Transport

Target Cells \& beam Stop

Muon Channels

General Site Construction

Engineering and Installation

Contingency

Total Estimated Cost

\section{Experimental Capital Equipment}

Muon Experimental Equipment

into the ultracold regime. The most intense source available has been at the IIL, where stored densities of $100 \mathrm{UCN} / \mathrm{cm}^{3}$ have been obtained. It has long been realized that a spallation neutron source provides the opportunity to produce and store UCN at the peak flux of the source, thereby increasing the intensity of UCN substantially. Cold neutron beams from a liquid hydrogen moderator at the Los Alamos Neutron Scattering Center (LANSCE) are presently available. Measurements of the cold neutron phase-space density at LANSCE, coupled with calculations of an optimized liquid hydrogen moderator and reasonable estimates of converting the cold neutrons into UCN using Doppler-shifted Bragg scattering, indicate that stored densities of approximately $600 \mathrm{UCN} / \mathrm{cm}^{3}$ can be expected. In addition, this source would also provide pulsed cold neutron beams (duration $-100 \mu$ s with 20-Hz repetition rate) with peak fluxes of a few $10^{9} \mathrm{n} / \mathrm{cm}^{2} / \mathrm{s}$ available at the end of a guide tube with an area of $50 \mathrm{~cm}^{2}$. Two guide tubes would be available, one fitted with a converter for UCN and the other for pulsed colci neutrons. The expected flux of such a facility is shown in Fig. VI-6. The cost is estimated to be roughly $\$ 3-4 \mathrm{M}$, and construction would require about two years. Such a facility would be unmatched in the world.

The physics program at this facility would revolve around several topics. Certainly measurements of the Electric Dipole Moment (EDM) of the neutron are of fundamental interest It is crucial to push the sensitivity to the neutron EDM down by several orders of magnitude. Current measurements are strongly limited by available source intensities (both in statistics and ability to study systematics) and substantial progress is contingent on

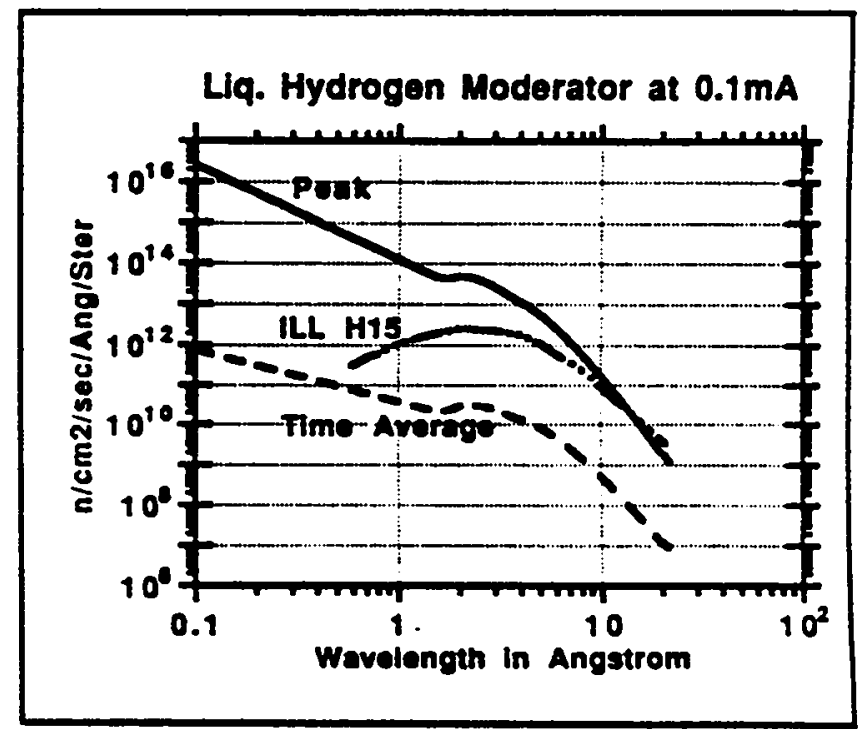

Fig. VI-6. Expected fluxes from an optimized LANSCE

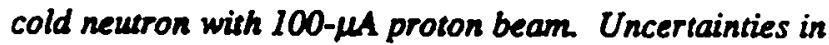
the moderator fluxes are approximately 10\%. The flux at ILL is shown for comparison. 
more intense sources. A LANSCE UCN source, possibly together with novel ideas involving interferometry in measuring the neutron EDM, offers an improvement in sensitivity by one to two orders of magnitude.

A second class of experiments that would be pursued involves $\mathrm{P}$ - and $\mathrm{T}$-violating experiments and measurements of angular correlations that could be carried out using the cold and very cold neutrons from this source. While the time-averaged fluxes available are low in comparison to reactor sources, a pulsed source offers several advantages. The time structure of the pulses from a LANSCE cold source would provide packets of neutrons roughly $10 \mathrm{~cm}$ in length at a $20-\mathrm{Hz}$ repetition rate. The time-averaged fluxes would be of order $10^{7} \mathrm{n} / \mathrm{cm}^{2} / \mathrm{s}$, which is high enough to provide acceptable counting rates. Since the length of each packet is relatively short. one can envision building detectors that would completely surround the neutron packet as it passes through the detector. This offers a great advantage in experiments looking for small asymmetries, as the end effects of the detector (which can be one of the limiting systematics) can be eliminated. Indeed, a number of experiments at reactors have chopped the beam (with a subsequent loss of intensity) in order to reduce problems from end effects. In addition, timing information allows one to strongly suppress $\left(-2 \times 10^{-3}\right)$ most backgrounds and to essentially eliminate backgrounds from beam polarizers, collimators, etc., which have been one of the major limiting elements in measurements of angular correlations.

Finally, there is a class of interferometric tests of quantum mechanical effects and tests of fundamental symmetries. Rather elegant experiments have been carried out using thermal and cold neutrons, which have observed the Aharanov-Bohm effect, gravitationally-induced quantum interference, the Sagnac effect, and the Berry phase. These and other similar experiments probe our understanding of quantum mechanics and gravitational interactions. For example, the first demonstration that the wave function for fermions changes sign under a rotation of $2 \pi$ was made using interferometry in a neutron beam. There is a real richness of ideas in this field, but limited access to UCN has hampered progress. Since the sensitivity of these experiments generally scales as $1 / \lambda$, the use of very cold and ultracold neutrons will significantly enhance the sensitivity of the next generation of these experiments.

Thus, with a rather modest effort, a source of cold and ultracold neutron beams with unmatched characteristics appears feasible in the near future at LANSCE. Such a source would fill the need identified by NSAC and provide the means to pursue a number of precision tests of fundamental interactions.

\section{A6 Upgrade}

\section{Introduction}

An initial design study has been completed for an upgrade to the $A 6$ beam stop on the LAMPF main proton beam line. This upgrade would allow a neutrino decay-inflight source and a helium-jet target experiment, and would be compatible with existing programs and proposed new initiatives.

The present beam stop accommodates stopped piondecay neutrino experiments, a radiation effects program, and isotope production for medical diagnostics and research. The original beam stop and isotope production system was built in 1973 . The beam stop was rebuilt in 1985-86 using a modular insert system inside a containment box. This arrangement allows for relatively easy reconfiguration, of beam stop components. The present beam stop receives $0.8 \mathrm{~mA}$ of $770-\mathrm{MeV}$ protons. This proton beam produces an isotropic stopped-pion neutrino source of about $5 \times 10^{14} \mathrm{v} / \mathrm{s}$. A neutron source of about $5 \times 10^{13} \mathrm{n} \mathrm{cm}^{-2} \mathrm{~s}^{-1}$ in the $5-$ to $20-\mathrm{MeV}$ energy range is available for radiation damage studies. The beam stop has served as the neutrino source for three major neutrino experiments: conservation of lepton number (E31), neutrino-electron elastic scattering (E225), and a search for neutrino oscillations (E645). There is presently a neutrino experiment (E1213) that will measure the neutrino capture cross section in ${ }^{37} \mathrm{Cl}$ and ${ }^{127} \mathrm{I}$. A new experiment using a large scintillation detector (LSND) is under construction and will be operational in 1993.

Programs that will benefit by the upgrade are the neutrino program, helium jet experiment, production of medical isotopes, radiation effects, accelerator production of tritium, accelerator transmutation of waste, fusion materials initiative, and production of ${ }^{37} \mathrm{Ar}$ for the calibration of solar neutrino experiments. The upgrade could be constructed as eariy as 1994 .

\section{Program Requirements}

The requirements of the various programs can be summarized as follows:

(A) The LSND neutrino program at LAMPF will search for oscillations by searching for the appearance of $\bar{v}_{e}$ from $\bar{v}_{\mu}$, the appearance of $v_{e}$ from $v_{\mu}$, and the disappearance of $v_{\mu}$. The program will also study the elastic scattering of $v_{\mu}$ from protons. A decay-in-flight facility at the A6 beam stop would allow a significant increase in neutrino research. 
A decay-in-flight source will increase the sensitivity for the appearance of $v_{e}$ from $v_{\mu}$ by about a factor of two. The source will increase rates for $v_{\mu}$ reactions by a factor of 5 , thereby making the $v_{\mu}$ disappearance and the neutrinoproton elastic scattering experiments possible.

The LSND neutrino detector sits approximately $12^{\circ}$ from the Line A direction, so the proton beam needs to be bent toward the detector with a magnet. The decay source should be about one meter in length and the pion production target about $30 \mathrm{~cm}$ in length. A target longer than $30 \mathrm{~cm}$ will not produce significantly more neutrino interactions in the detector since the energy of the protons and of the outgoing pions is degraded. A longer decay path will produce more neutrinos but will also introduce $\vec{v}_{e}$ backgrounds from $\mu^{-}$decays.

(B) The helium-jet experiment is the first step toward an IsoSpin Laboratory (ISL) proposal. This thin target approach is expected to have several advantages over a thick target: The reduced transport time of ions enables higher yields of short-lived ions, the amount of radioactivity produced is reduced, and the high-maintenance ion source is decoupled from the high radiation field. The goals of the He-jet experiment are: (1) prove that the He-jet target can operate reliably for several months in the LAMPF beam stop; (2) measure yields for a variety of radionuclides. especially for nonvolatile elements; (3) couple the He-jet to a fast radiochemical separation system to do nuclear structure studies of neutronrich Tc, $R u, R h$, and $\mathrm{Pd}$; and (4) measure electromagnetic moments of neutron-rich $\mathrm{Z}=$ 40 - 46 nuclei using off-line low-temperature nuclear orientation.

(C) The isotope production program needs as much proton and neutron flux as possible but is not particularly sensitive to energy. The hardware should provide easy access for removing short- lived isotopes and provide from nine to fifteen irradiation ports with independent access.

(D) The radiation effects program uses both protons and neutrons. The proton irradiations need only space in the beam while the neutron irradiations need an intense and stable source of 5- to 20$\mathrm{MeV}$ neutrons. The initiatives for fusion materials studies would like to upgrade the neutron source by a factor of ten. The accelerator production of tritium (APT) and accelerator transmutation of waste (ATW) programs both have similar requirements.

\section{Reference Design}

A reference beam stop design has been developed that will accommodate the needs of the existing programs and new initiatives; it is shown in Fig. VI-7. The beam stop upgrade is expected to cost less than $\$ 1.5 \mathrm{M}$. Some of the programs have not defined their needs well enough for a detailed design to be made. However, the modular design of the beam stop will allow devices to be placed in the location of the present A6 beam stop without compromising the neutrino or the helium-jet experiments.

The neutrino program needs the highest-energy portion of the beam and requires the beam to be bent toward the LSND detector. This is accomplished in the reference design by placing a bending magnet followed by a $30-\mathrm{cm}$ water target upstream in the main containment box. The slight loss of proton beam energy and intensity will not significantily affect the isotope production and radiation effects programs.

The helium-jet target is relatively small $(5 \mathrm{~cm}$ diameter and $20 \mathrm{~cm}$ long) and thin $(2-4 \mathrm{~g} / \mathrm{cc})$ so that it can be placed upstream of the other experiments without disturbing the beam. The present isotope production system can be greatly simplified and compacted. Detailed designs are in progress. Conceptual designs for a hydraulic rabbit system were carried out in 1987 and it is expected that such a system can be used at the new beam stop location. 


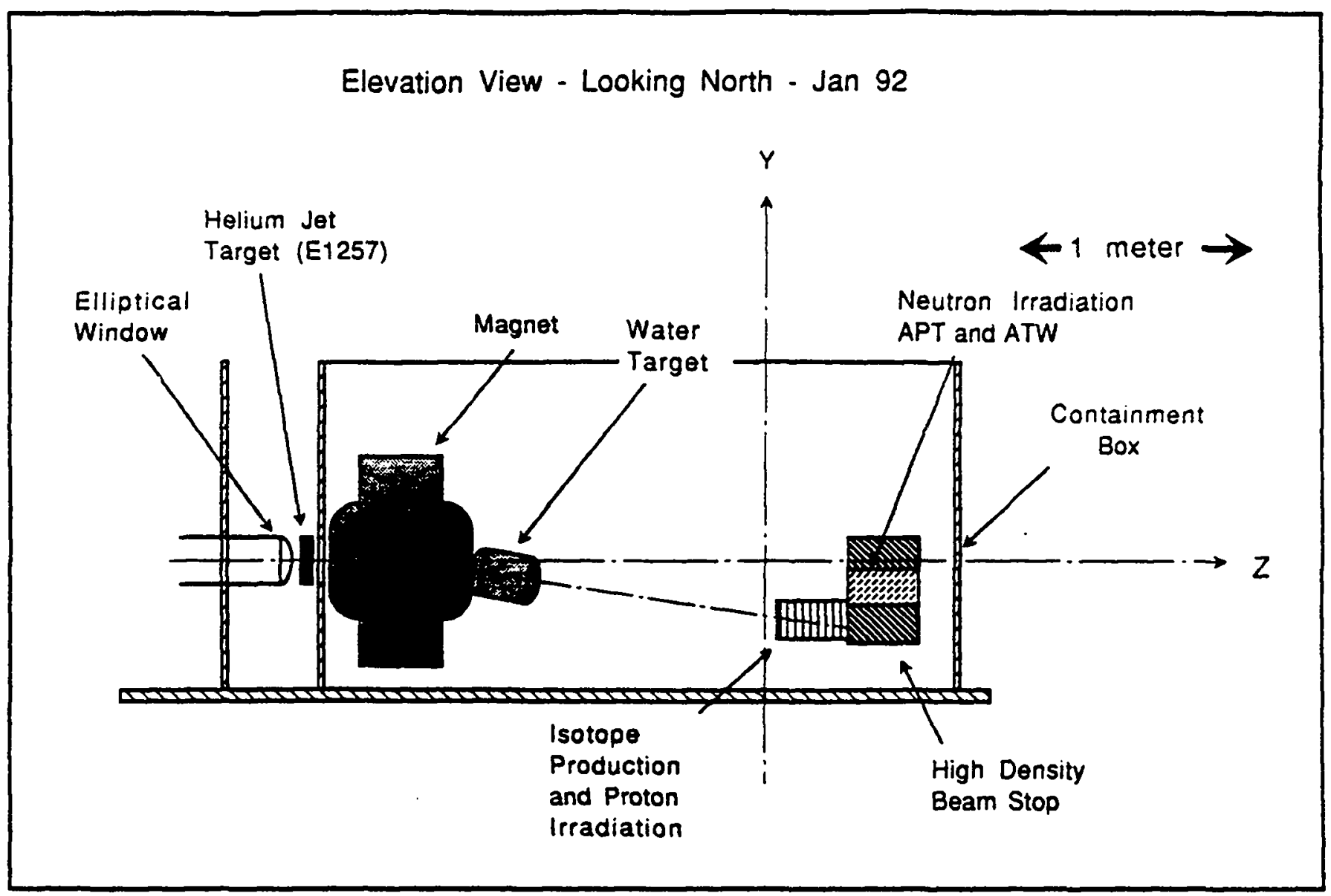

Fig. V1-7. Reference design for the proposed A6 beam stop upgrade. 


\section{Appendix A}

\section{Selected List of LAMPF Research Highlights for the Past Five Years}

- Observation of a clear signature of NN correlations in nuclei from low-energy pion double charge exchange.

- Setting of the most sensitive limits for mixing between lepton families in the rare processes $\mu \rightarrow \mathrm{e} \gamma$, $\mu \rightarrow \mathrm{e} \gamma$, muonium-antimuonium transitions, and in neutrino oscillations.

- Measurement of quasifree $(p, n)$ spin transfer observables providing essential data to test models of pion collectivity in nuclei.

- Observation of large parity-violating effects in compound nuclei using epithermal neutrons.

- Evidence for a neutron halo and observation of possible new excitation modes in the exotic ${ }^{11} \mathrm{Li}$ nucleus produced in pion double charge exchange.

- Measurement of the parameters of muon catalysis of the D-T fusion cycle.

- Determination of the $I=0,1$ nucleon-nucleon elastic scattering amplitudes up to $800 \mathrm{MeV}$.

- Study of multiphoton detachment, and detachment of both electrons with one photon, in $\mathrm{H}^{-}$ions.
- Studies of multinucleon pion absorption showing their increased importance at higher energies.

- Observation of the interference between weak neutral and charged current amplitudes in ve elastic scattering.

- Characterization of $\Delta$ - nucleus interactions in pion elastic, inelastic and charge exchange scattering.

- Demonstration of energy shifts in quasifree and $\Delta$ excitations in nuclei from comprehensive $(p, n)$ data.

- Characterization of chiral symmetry breaking in low energy $\pi \mathrm{N}$ scattering.

- Measurement of the masses of over 60 neutron-rich isotopes from ${ }^{11} \mathrm{Li}$ to ${ }^{66} \mathrm{Fe}$.

- Precision measurement of the muonium hyperfine structure interval and the ratio of the magnetic moments of the muon and proton.

- Observation of the Giant Isovector Monopole Resonance and Double Giant Resonances in single and double pion charge exchange reactions. 


\section{Appendix B}

\section{LAMPF Facility Milestones}

Official Ground Breaking

5-MeV Beam Achieved

100-MeV Beam Achieved

211-MeV Beam Achieved

800-MeV Beam Achieved

Dedication to Senator Clinton P.

Anderson

First $\mathrm{H}^{-}$Injector Beam

First Simultaneous $\mathrm{H}^{+}$and $\mathrm{H}^{-}$Beams

Beam to Area B

First Experiment (\#56) Received Beam

First Meson Production, Beam to

Area A

First Medical Radioisotope Shipment Usable $100 \mu$ A Beam to Switchyard

First Experimental Pion Radiotherapy

Start of Area A Rebuild

Accelerator Tumon

Dual-Beam Capability, 100- $\mu \mathrm{A} \mathrm{H}^{+}$ and $3-\mu \mathrm{A} \mathrm{H}^{-}$, Demonstrated

First Pions Through EPICS

$100-\mu$ A Production Beam in Area A

Startup of Isotope Production Facility

HRS Operation Begins

Maintenance by "Monitor" System of Remote Handling

Proton Beam to WNR

Polarized-Proton Beam Available

Experimental Results at Neutrino Facility

Cloud and Surface Muon Beams: SMC

EPICS Operation Begins

300- $\mu$ A Production Beam in Area A

$\pi^{0}$ Spectrometer Begins Operation

Operation of Polarized-Proton Target

Successful Water-Cooled Graphite

Production Target

$600-\mu$ A Production Beam in Area A

Commercial Production of

Radioisotopes
February 15, 1968

June 10, 1970

June 21, 1971

August 27, 1971

June 9, 1972

September 29, 1972

March 28, 1973

May 4, 1973

July 15,1973

August 24, 1973

August 26, 1973

July 30,1974

September 5, 1974

October 21, 1974

December 24, 1974

August 1, 1975

September 14, 1975

March 18, 1976

August 1976

October 15, 1976

November 1976

Fall 1976

March 12, 1977

April 1977

July 1977

July 1977

August 1977

Fall 1977

February 1978

Spring 1978

November 1978

November 1979

January 1980
Spin Precessor Added to EPB

Variable-Energy Operation

Focal-Plane Polarimeter Operational at HRS

Safety Award to LAMPF Users Group, Inc., for Working One Million Man-Hours Since 1975 Without

a Disabling Injury

750- $\mu$ A Production Beam in Area A

Staging Area Constructed

1.2-mA Beam Capability Demonstrated

Duty Factor $\geq 9 \%$ Achieved

Clamshell Spectrometer On Line

High-Intensity $\mathrm{H}^{-}$Injector Operational

New Switchyard Permits Three-Beam Operation

Proton Beam to Proton Storage Ring (PSR)

1-mA Production Beam in Area A

Polarized Beam Delivered with 10-MHz Micropulse Structure for Neutron Timing

Neutron Time-of-Flight Facility ShortFlight-Path Commissioned (New Beam Line in NPL)

Linac Rebuncher Scheme Implemented to Produce Time-Focused Micropulses at WNR and NTOF

Operation with Three Beam Energies to

Line $A$, Line $D$, and Line $X$

Polarized Nuclear Target $\left({ }^{13} \mathrm{C}\right)$ Used on HRS

Commissioned MRS Achieving 1.3MeV Resolution at $800 \mathrm{MeV}$

First Polarized Beam to Experimental Area from New Optically Pumped Polarized Ion Source (OPPIS)

OPPIS Exceeds Design Goals
February 1980

June 1980

October 1980

October 27, 1980

1982

1982

February 7, 1983

February 1984

June 1984

April 1985

April 1985

May 1985

Summer 1985

October 1986

November 1987

November 1987

Fall 1987

June 1988

July 1989

September 1989

June 1990 